Tause on the Wocument:
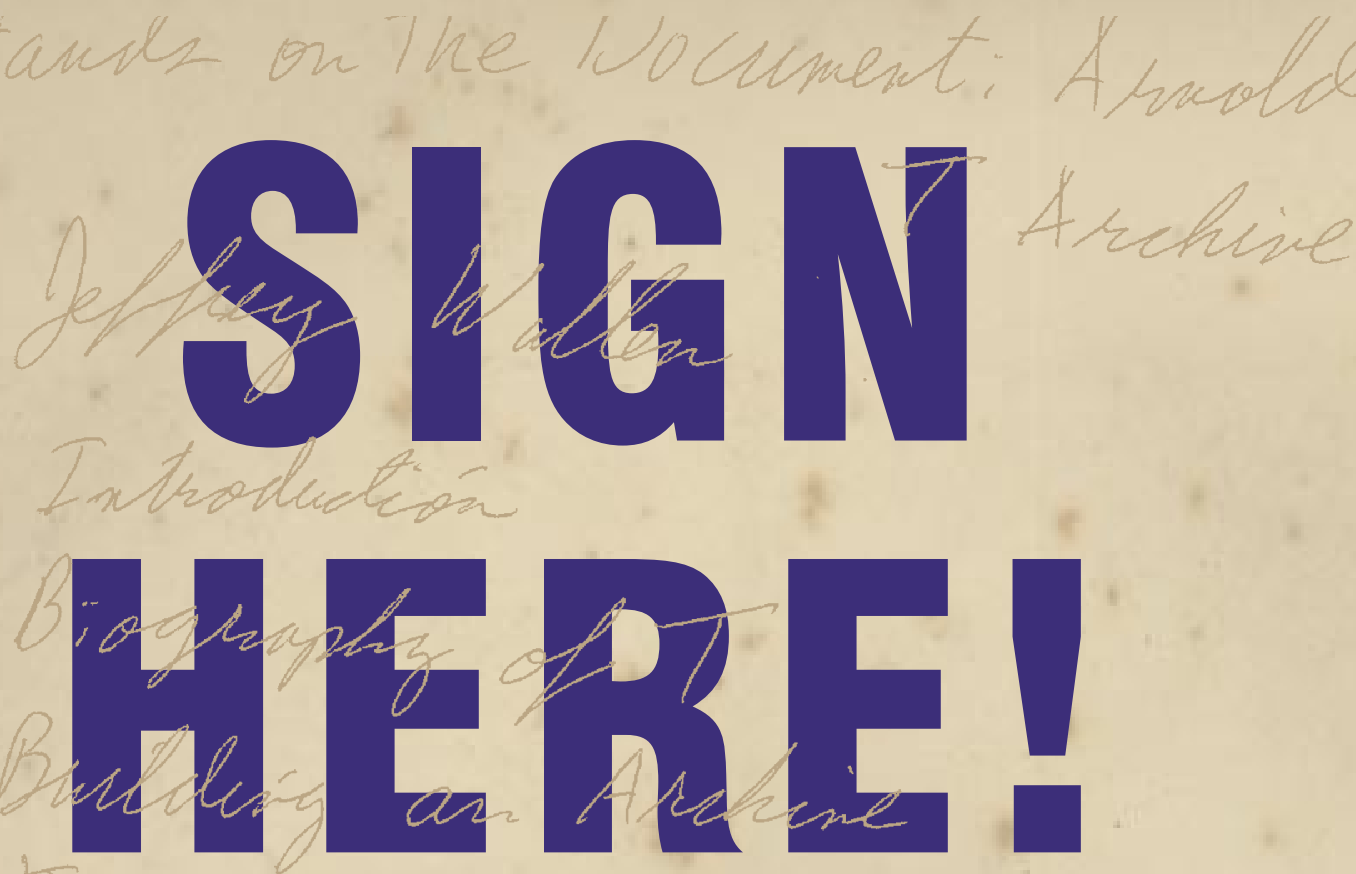

\title{
Handwriting in the Age of New Media
}

installations and Exhibituoins

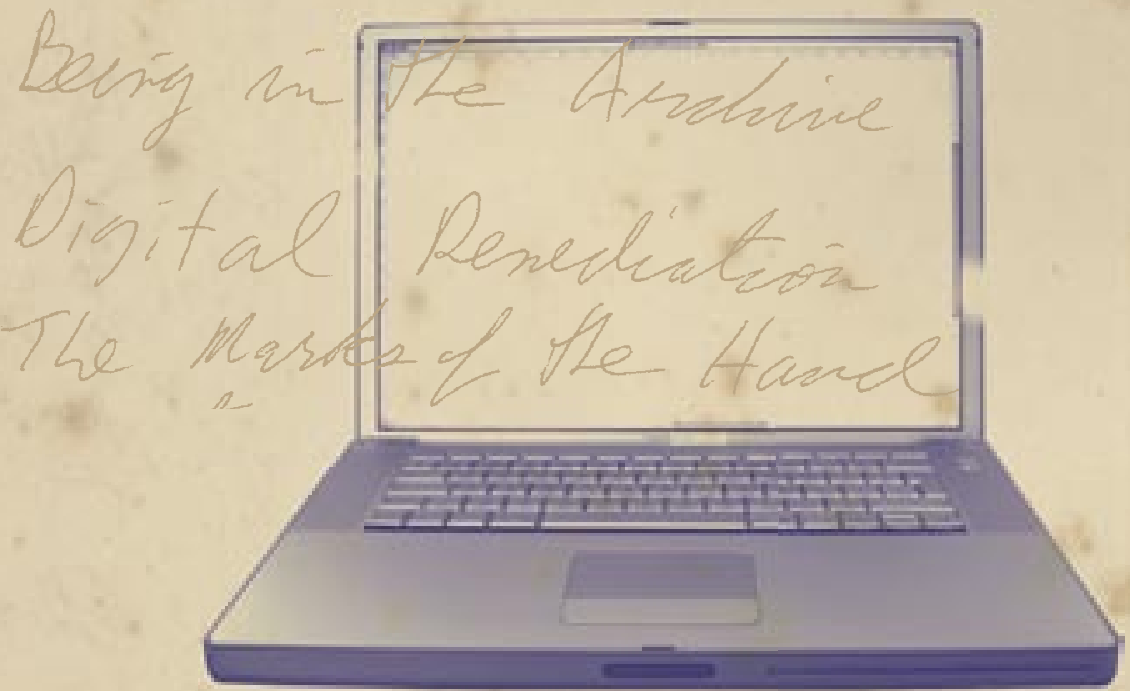

Edited by Sonja Neef / José van Dijck / Eric Ketelaar Amsterdam University Press 
Sign Here! 


\section{Transformations in Art and Culture}

The series Transformations in Art and Culture is dedicated to the study of historical and contemporary transformations in arts and culture, emphasizing processes of cultural change as they manifest themselves over time, through space, and in various media. Main goal of the series is to examine the effects of globalization, commercialization and technologization on the form, content, meaning and functioning of cultural products and socio-cultural practices. New means of cultural expression give meaning to our existence, and give rise to new modes of artistic expression, interaction, and community formation. Books in this series will primarily concentrate on contemporary changes in cultural practices, but will always account for their historical roots.

The Publication of this book is made possible by the Dutch Research Organisation, Nwo

Cover design and layout Frederik de Wal, Schelluinen

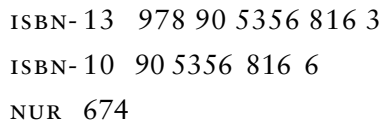

๑) Amsterdam University Press, Amsterdam, 2006

All rights reserved. Without limiting the rights under copyright reserved above, no part of this book may be reproduced, stored in or introduced into a retrieval system, or transmitted, in any form or by any means (electronic, mechanical, photocopying, recording or otherwise) without the written permission of both the copyright owner and the author of the book. 


\section{Sign Here! Handwriting in the Age of new Media}

Edited by Sonja Neef, José van Dijck, and Eric Ketelaar 
Sign Here! Handwriting in the Age of Technical Reproduction: Introduction

José van Dijck and Sonja Neef

Section One: Authentic Copies

23

Authentic Events:

The Diaries of Anne Frank and the Alleged Diaries of Adolf Hitler Sonja Neef

51

The Authority of Drawing:

Hand, Authenticity, and Authorship

Michael Wetzel

60

Authenticity and Objectivity in Scientific Communication:

Implications of Digital Media

John Mackenzie Owen

76

Authenticity in Bits and Bytes

Hannelore Dekeyser

Section Two: Re-Mediating Handwriting

95

Signature Identity Content:

Handwriting in an Age of Digital Remediation

Richard Grusin

116

Writing the Self:

Of Diaries and Weblogs

José van Dijck

134

Hands on the Document:

Arnold Dreyblatt's T Archive

Arnold Dreyblatt and Jeffrey Wallen 
150

Faithfully Submitted:

The Logic of the Signature in Marcel Proust's A la recherche

Mieke Bal

164

(Hand)writing Film History:

Saul Bass Draws Martin Scorsese in a Title Sequence and Writes

his Name Underneath

Rembert Hüser

Section Three: Handwriting and (Dis-)Embodiment

183

Writing on Archiving Machines

Eric Ketelaar

196

Blood Samples and Fingerprint Files:

Blood as Artificial Matter, Artistic Material, and Means of the Signature

Thomas Fechner-Smarsly

206

Writing Over the Body, Writing With the Body:

On Shirin Neshat's Women of Allab Series

Begüm Özden Firat

221

Perfor/m/ative Writing:

Tattoo, Mark, Signature

Sonja Neef

237

Contributors

241

Illustration Acknowledgement

242

Index 


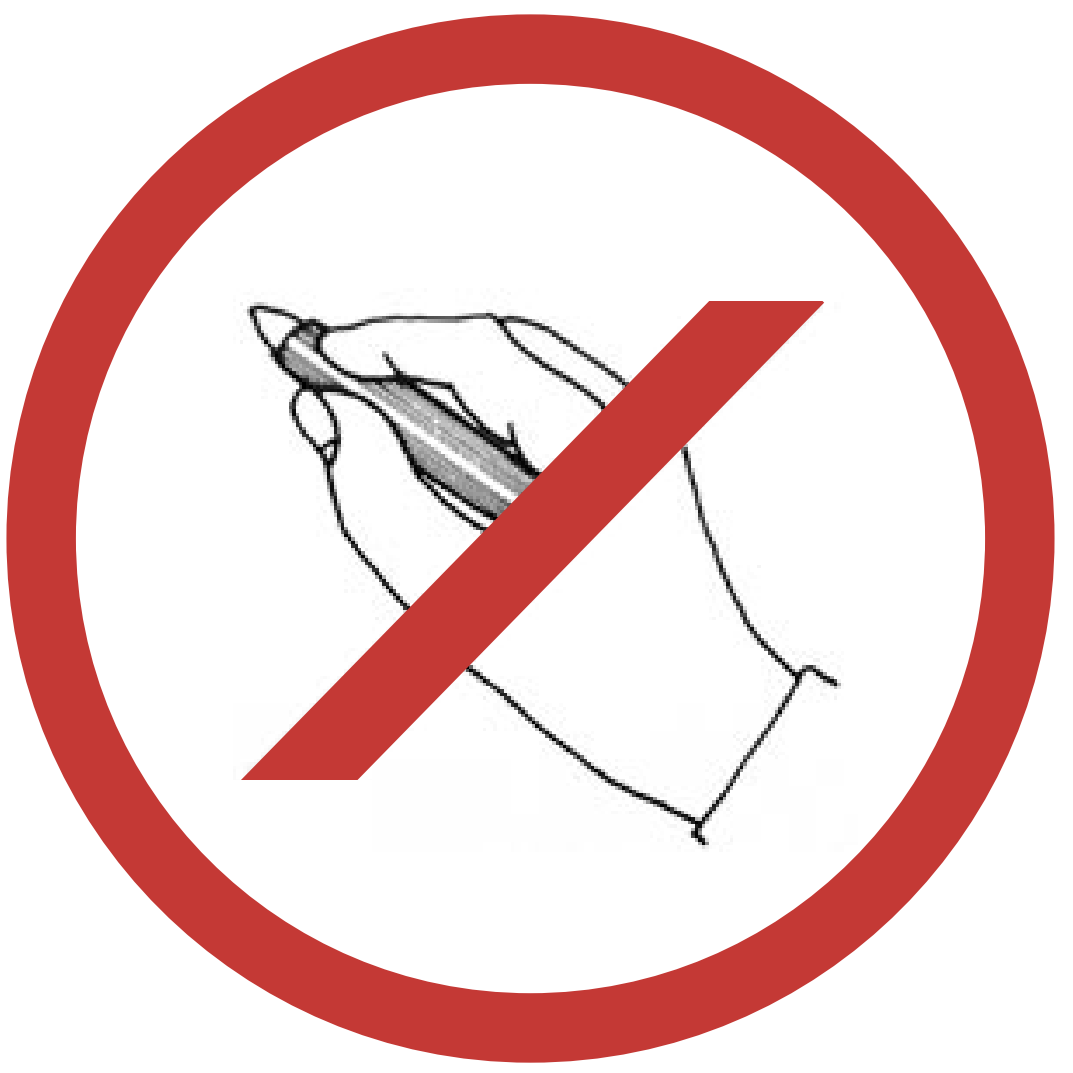

1. The 'please-do-not-write-by-pen'-sign 


\section{Sign Here!}

\section{Handwriting in the Age of Techical Repooluction: Introduction}

On some computer screens, particularly large projection screens used for classroom presentations, you may find a peculiar pictogram, showing a right hand holding a pen, crossed out by a bold red diagonal line - not unlike a non-smoking sign - conveying the warning 'do not write here'. ${ }^{I}$ Against a backdrop of an oldfashioned blackboard in the seminar room, the sign takes on a historical meaning. Indeed, we only rarely write with chalk or pen these days, as our private desktops and public classrooms are inundated with computers and media equipment, and our writing tools mostly consist of keyboards, screens, and projectors. Handwriting, in the age of the Internet and digital media, is considered by many to be a backward technique, a slowly deteriorating and gradually vanishing tradition, and the myth of its decline is as widespread as the pictogram. After all, the sign addresses only those rare specimens of the human race who are, as of yet, unfamiliar with the very basic principles of multimedia. Like the non-smoking sign, 'writing without a pen' is now supposed to become the default mode in our contemporary classroom.

Ever since the invention and spread of moveable type in modern times and of the typewriter in the late-19th century, the idiosyncrasy of manual writing has given way to standardized, replicable, power-driven letters produced by machines. With the advent of word processors, the significance of 'hand' in conjunction with 'writing' is expected to diminish even further, as the cultural emphasis on digital flexibility and infinite manipulation is displacing values traditionally attached to handwriting, such as authenticity, uniqueness, and personality. But despite the 
widespread and invasive mechanization of writing, the power and meaning of handwriting goes way beyond its standardizing instruments of inscription. Handwriting has given rise to a number of cultural practices, such as letter writing, and cultural forms, such as diaries or Post-it notes. Moreover, handwriting is also an aesthetic category that we still uniquely associate with a manual craft: from calligraphy to urban graffiti, from tattooing to signing, the physical, the human hand is pivotal in the production of letters and texts.

Will handwriting actually disappear in the age of new (digital) media? In fact, we argue in this volume that this question is philosophically and historically incorrect. It is philosophically erroneous because it presumes a teleological relationship between media and their ensuing practices and forms. Technologies, cultural practices and forms, however, always change in conjunction with each other, and although specific apparatuses may be displaced in the course of time, related forms and practices hardly ever vanish. If we look at the history of handwriting, we may notice that in spite of the emergence of generations of 'writing machines', manual script has never disappeared; on the contrary, as it evolved, handwriting adjusted its practical functions, social meanings and cultural aesthetics. The introduction of the typewriter, for instance, shifted the emphasis to the standardization of script, but it may even have increased the notion of authenticity associated with handwriting. The invention of machines like the Xerox copier or the digital scanner once again shifted the use and meaning of handwriting by enabling the reproduction of individual handwriting, and, in the case of Optical Character Reading (OCR) as it is also used in the latest invention of the tablet PC, the automatic transcription of manual script into typeface. In addition, technologies that are not immediately script-related, such as photography and film, also affected the cultural meaning of handwriting, if only because these technologies allowed for an exact 'recording' of the manual act or its product. Handwriting, in other words, has never disappeared in the wake of new technologies, but has always adjusted its use and meaning in the face of larger technological, social, and cultural transformations. It is therefore hard to believe that handwriting will vanish, as long as its technologies are intimately tied to particular cultural practices and forms that are continued in the present.

Technologies, forms, and practices are inscribed with cultural values that change along with larger social and cultural transformations. Important cultural concepts, such as original and copy, authenticity, reproducibility, uniqueness, or iterability, are never anchored once and for all because the cultural value of these concepts shifts with every innovation or transformation. For instance, in the 19th century, the meaning of 'uniqueness' metamorphosed in the wake of apparatuses enabling mechanical reproduction. By the same token, the concept of 'authenticity' is currently undergoing a substantial overhaul now that computers are becoming the preferred tools for (written) communication. Signatures, for instance, were supposed to be authentic, idiosyncratic signs of selves, intimately tied to the hand that produced them; now that they are gradually replaced by memorized codes and biometrical scans, the former handwritten sign of identification is no longer considered foolproof. The exchangeability of original and copy prompts us to re- 
consider conventional notions of manuscripts: what counts as an original 'document' in the current digitized office environment? And why do we value handwritten manuscripts as 'authentic' proofs of historical persons or events? Every new technology not only affects our everyday habits and practices in using them, but also situates handwriting in a new perspective, thus requiring a reinterpretation of its meaning.

This collection of essays will address three aspects of handwriting in the age of new media: authenticity, remediation, and (dis)embodiment. Each of these aspects will be further elucidated in the paragraphs below.

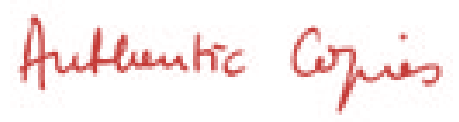

The articles collected in the first section of this volume focus on an aspect of handwriting that is dramatically challenged by mechanical and electronic reproduction practices: its claim to authenticity. Handwriting is traditionally regarded as an autography, as an un-exchangeable, unique and authentic 'signature' that claims to guarantee the presence of an individual writer during a historically unique moment of writing. ${ }^{2}$ This claim for authenticity distinguishes handwriting from its cultural opposite, mechanical writing, in the sense of print or typed writing. After all, the cultural significance of mechanical writing resides in its capacity to be iterable and reproducible. The reproduction of authentic handwriting, on the other hand, risks being considered a forgery. This view on handwriting has a long tradition in different disciplines, most importantly in jurisprudence, but also in historical studies of original sources, and in art theory, where it delineates the status of the artist/author. In connection with the 20th-century's technological developments, the idea of the uniqueness of the signature has been challenged philosophically, most profoundly by Derrida in his famous essay 'Signature, Event, Context'. Derrida claims that the signature is a performative sign; its validity - or as Austin would say, its 'felicity' - is grounded in the fact that the signature is a singular event, and that it repeats or quotes a set of norms constituting a cultural or juridical context.

This section explores how the double structure of a signature as both singular and iterable needs to be redressed in the wake of technological reproduction. How do the concepts of uniqueness and iteration, of authenticity and counterfeit in relation to handwriting, change when the binary opposition 'authentic' vis-à-vis 'copy' no longer appears to be anchored in the distinctive materiality of the sign? And what are the consequences for both historical and contemporary cultural forms and practices still hinging on modernist notions of authenticity?

As a common starting point for the articles collected in this section, we chose Walter Benjamin's famous article 'The Work of Art in the Age of Mechanical Reproduction'. His article is still challenging, not only because it touches on the complexity of the problem of authenticity and its interrelations with notions of uniqueness and originality, but also because it anticipates many of the more recent theoretical debates about cultural objects in the age of new media, including digital me- 
dia. In addition to putting contemporary debates in a historical perspective, it also renders the concept of authenticity relevant if not center stage for the disciplines represented in this volume.

In his renowned piece, Benjamin investigates the art status of reproduced artworks. Original artworks, such as paintings, are singular and durable, and they therefore have an 'aura' connecting them to a certain tradition. For Benjamin, authenticity relates to the here and now of the original artwork, 'its presence in time and space, its unique existence at the place where it happens to be' (220). Reproduced artworks, on the other hand, are regarded as iterable and transient, they lack this 'aura', as they primarily serve 'the desire of contemporary masses to bring things "closer" spatially and humanly, which is just as ardent as their bent toward overcoming the uniqueness of every reality by accepting its reproduction' (223). Unfortunately, Benjamin does not elaborate on the 'auratic' status of writing in his essay. The aim of this section, in a way, is to fill that gap. For now, we will tentatively argue that, according to Benjamin's classification of cultural objects, writing should be considered non-auratic. The basic principle of writing, after all, is repetition. The alphabet, a discrete number of distinct letters, is standardized for the sake of iteration and of mechanical reproduction - first by Gutenberg's movable type, later by the typewriter, and, most recently, by electronic media. It was for good reasons that Friedrich Kittler, in Gramophone, Film, Typewriter, categorized the typewriter as referring to Lacan's symbolic system:

Only the typewriter provides writing as a selection from the finite and arranged stock of its keyboard. It literally embodies what Lacan illustrated using the antiquated letter box. In contrast to the flow of handwriting, we now have discrete elements separated by spaces. Thus, the symbolic has the status of block letters. (28-29) 3

We will argue in response to Kittler that handwriting, in spite of its fluidity, is still bound up with the symbolic system of language - in the sense of a system of differences - and as such, it is spellable and repeatable. Still, it does make sense to contrast, as Kittler does, handwriting with its cultural opposite, typed writing, to explore the 'auratic' dimension of handwriting that resides in its authenticity. Let us tentatively describe the authenticity of handwriting by means of three criteria marking the boundaries between handwriting and typed writing, that is, singularity, individuality, and materiality.

Singularity. A typed text may be printed in an unlimited number of ways and in an infinite variety of materials without it directly influencing its authenticity. Written text is reproducible, printable, and, as Nelson Goodman states in his Languages of Art, 'allographic', or 'spellable'. ${ }^{4}$ The reproduction of handwriting, however, requires reproduction technologies that are closer to those of images than to those of writing. Handwriting, therefore, functions like painting or drawing, which are categorized by Goodman as 'autography' because their central quality resides in their uniqueness. Even though the basic principle of all writing is iteration, for handwriting there is no such thing as two manuscripts that look exact- 
ly the same - except if one is a perfect Xerox copy of the other. But then, it can be expected that one will wish to distinguish between the original, 'auratic' version and the copy.

Individuality. The authenticity of handwriting, as opposed to typed writing, resides also in its potential to refer to an un-exchangeable individual. Handwriting is regarded as auratic because of its capacity to function as a signature that claims to guarantee the presence of an individual writer during a historically unique moment of writing. Whereas in typed writing, as Martin Heidegger (119) has argued, 'every person looks the same', for handwriting, there is no such thing as two individuals writing identically. ${ }^{5}$ Except, of course, in Goethe's novel Wablverwandtschaften, in which the lovers Eduard and Ottilie, miraculously enough, used the same handwriting (cf. Hörisch, chapter I/2).

Materiality. This subjectivity, then, is physically inscribed in the movement and the pressure of the pen led across the paper, leaving there an un-exchangeable, personal trace. If handwritten manuscripts are regarded as 'auratic', their 'aura' resides precisely in their material authenticity incorporating the undividable 'here and now' of the manuscript's historical origin.

The problem central to this first section of the book can be grasped by the example of the pentagraph, a multiple writing machine that made its appearance at the end of the 18th century (Illustration 2). James O'Toole describes the functioning of this machine as follows: 'The pentagraph was constructed so that, as the writer moved one pen along a sheet of paper, another pen, attached to it by wooden arms, wrote the identical words on a second sheet' (643). This writing practice exemplarily raises the questions central to this first section: who is writing here? Which one of these handwritings is the original? And which one is the duplicate copy? Can they both be unique? And authentic?

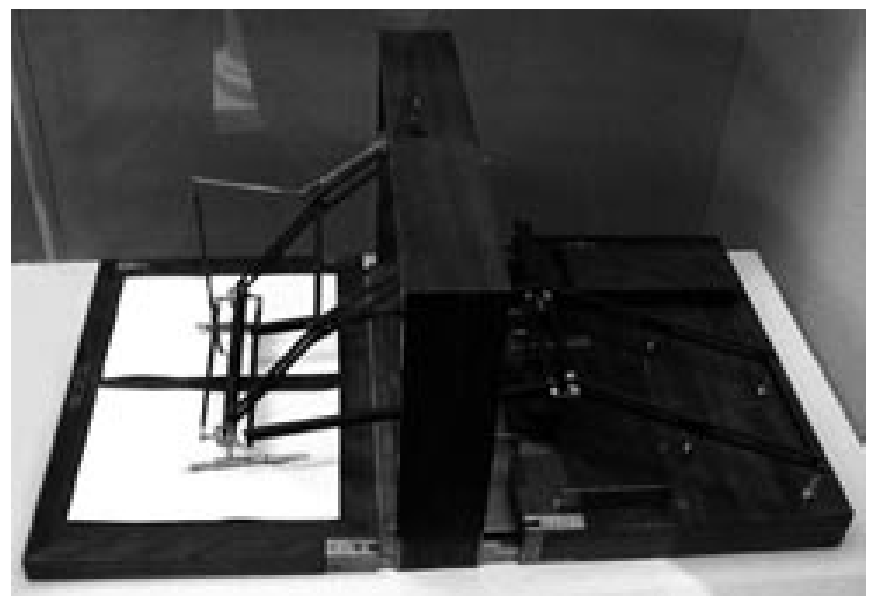


Contributors to this section of the volume will both accentuate and question the distinction between handwriting and typed writing, autography and allography, original and copy, in relation to various types of handwriting. Sonja Neef addresses the problem of cultural memory of an 'authentic' past by discussing the contested 'authenticity' of the historical, handwritten diaries of Anne Frank and the alleged diaries of Adolf Hitler. Michael Wetzel discusses the concept of the hand by bringing it in dialogue with the ideas of authenticity and artistic authority as performed in the work of Marcel Duchamp; in his work, handwriting is distinctly linked to the logic of the 'trait'. The end of 'originality' is also proclaimed by John Mackenzie Owen, who relocates the issue of 'authorship' to a space where the 'aura' of authentic handwriting is literally volatilized in electronic impulses: the digital world. Within this digital space, and finally, Hannelore Dekeyser attempts to save what seems to have vanished in the process of digitization: the signature's authenticity that, even though it is deprived of its auratic dimension, can function perfectly as a legal instrument.

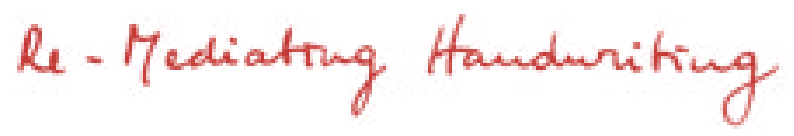

For handwriting, seen as a Western cultural practice emerging from an age-long tradition, the primal writing implement has been the stylus, a tool that started as a wedge to incise traces in sand, in clay, or in wax. Over time, the stylus took on different shapes: as a brush which, once turned around, became a quill, then a pencil, a pen, and a ballpoint pen. In all these forms, the stylus was led by the hand, particularly by the right hand, and its basic product was a line or a 'trait'. In our contemporary communication society, the archaic stylus is to a large degree replaced by the typesetting tool of the keyboard. Paradoxically, the most threatening and - literally - most stirring threat to handwriting, electricity, turned out to be the vehicle that initiated a renaissance in handwriting. Technical print media and visual media enable the reproduction of images and thus of handwriting: photography, film, microfiche, Xerox, fax, scanner, and computer. On the one hand, these media supplant handwriting, on the other hand, they bring handwriting back to us, but in a different - what Bolter and Grusin call - a 'remediated' form. The central question of this section is to discuss handwriting in its relation to a second medium in which it is depicted, reproduced, or remediated. Hence, this section has a double focus: it is devoted both to the 'mediation' and to the 're-mediation' of handwriting.

Mediation. In the history of writing theory, from Plato's Kratylos to Saussure's Cours linguistique générale, writing has been regarded as derivative of speech. Until this very day, writing is defined as a sign system with a referential structure connecting writing to language and speech, and the relationship between sign and reference is described as arbitrary. Writing is specified as a system of difference, and this system is said to be productive, meaning that a finite stock of signs can produce infinite varieties of articulations. These philosophical and linguistic epistemes define writing's essential characteristics - its referential structure, its arbitrariness, 
its functioning as a system of difference, and its productivity - without taking its medial or material mode into consideration.

Writing is indeed closely related to language and speech, but not exclusively. Writing is also and primarily a visual medium, rendering it complex as a system based both on the articulation strategies of alphanumeric text and of visual images. This visual dimension distinguishes standardized mechanical writing from handwriting, which is idiosyncratic and often risks being illegible. This specific materiality qualifies the handwritten text as allographic and autographic at once; its semiotics unfolds in this in-between-media, as 'text-image' or as 'image-text'. Moreover, handwriting as a specific form of writing emphasizing the individual dimension of it, finds an audible pendant in the voice, which is as un-exchangeable as handwriting. Because of this audible dimension, handwriting may also appear as a 'sound-image'. In conclusion, handwriting is 'mediated' because it is a hybrid medium composed of visual (writing), audible (speech), and verbal (language) media. Handwriting's hybrid structure becomes particularly visible when it is incorporated in another medium - when it is thus literally re-mediated.

ReMediation. In their now classic book on new media and digital culture, Jay Bolter and Richard Grusin (1999) have developed the concept of 'remediation' to trace how new types of media re-interpret and concurrently re-form other technologies and their uses. In this section, we deploy the concept of 'remediation' to examine the transformation of cultural practices and forms conventionally associated with manual writing, in order to explore what happens when 'script' is performed in a second medium. Bolter and Grusin's concept of 'Remediation' will be reformulated as 'ReMediation'; the capital ' $M$ ' producing two interrelated concepts of 'mediation' and 'remediation' is introduced to indicate that handwriting is a double-edged practice. Handwriting is itself mediated because it is grounded in the inscription technologies of other media, and, in turn, it remediates other media when it becomes a model for 'newer' media effectively intervening in the old ones, for example when the archaic stylus is resurrected as an electronic tool of the tablet PC.

Our current media culture is full of 'ReMediated' handwriting. We find it etched, photographed, Xeroxed, and digitally scanned. Intelligent Fond Analysis (IFA) allows us to write our own handwriting via a keyboard, to perform handwritten e-mail correspondence, indeed, even to write someone else's 'hand'. For example, Leonardo da Vinci's left-handed mirror writing, today still regarded an attribute of the genius, can be performed on a keyboard by using a free online transmitter (Illustration 3). ${ }^{6}$ The focus of this section is the examination of handwriting at those loci where it is least expected: in digital environments. We will analyze how it gets 'incorporated' by a digital apparatus that privileges multimedia expression while erasing material signs of historicity and personality, but also in other environments where handwriting's inscription technologies are remediated: film and literature. 

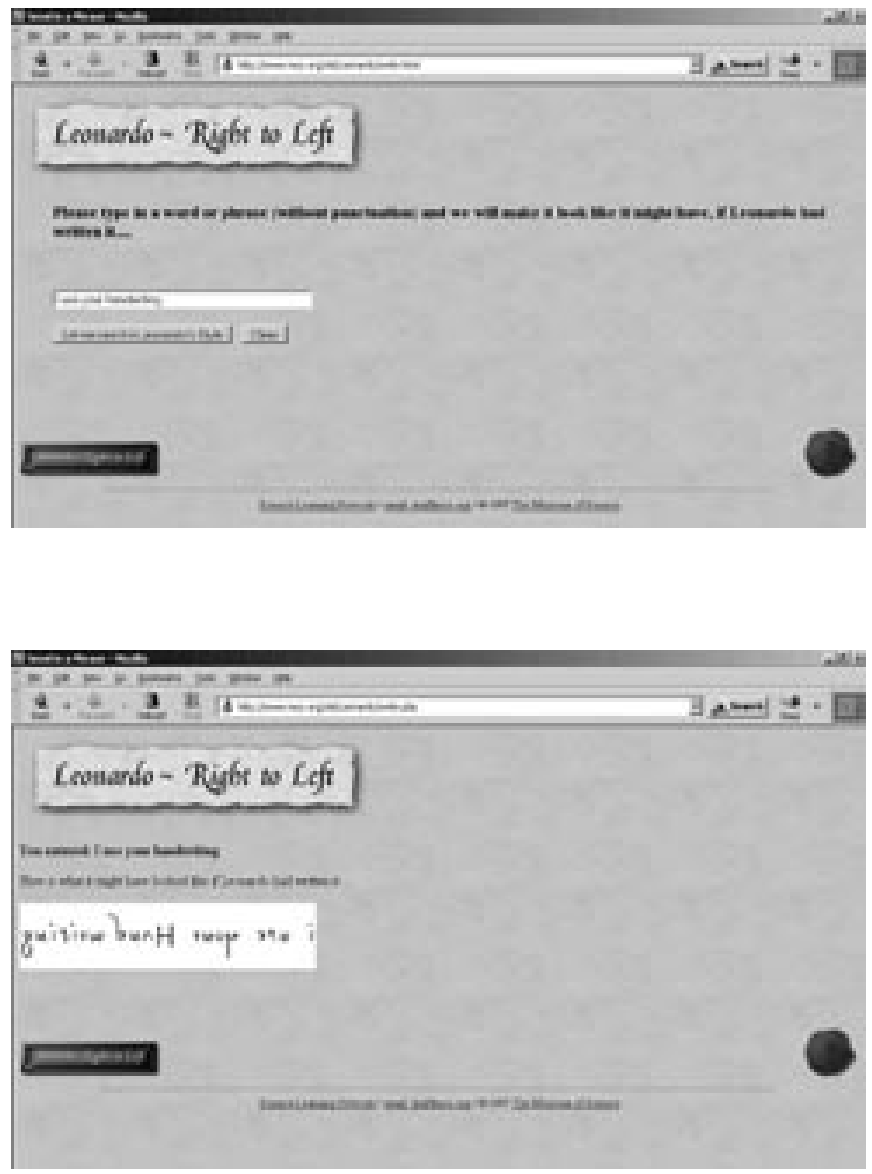

3. The Leonardo-Right to Left website, March 2005

The contributions in this section map out some implications for the ReMediation of traditional uses of handwriting. Richard Grusin reconsiders the status and meaning of the signature as a legal and cultural means to proving someone's individuality, at a time when audio-visual 'mediations of self' are taking center stage. José van Dijck discusses the cultural meaning of weblogs in relation to the former practice of diary writing. How do former notions of intimacy and personality change when the diary is transformed into a multi-medial form characterized by immediacy and public exposure? Arnold Dreyblatt and Jeffrey Wallen, then, focus on the effects that the specific materiality of handwriting has on the archive by analyzing the ReMediation of personal documents of a historical figure - a suspected Eastern European spy found in archives around the world. Dreyblatt's art installation The T Project chal- 
lenges us with poignant questions concerning the migration of lives and (handwritten) texts across different media. Mieke Bal takes us to literary studies, where she investigates the logic of handwriting in Proust's A la recherche du temps perdu in a double sense; the material reality of Proust's idiosyncratic handwriting functions as a medium that produces the diegetic reality of the second medium of literature, which, in itself ponders the status of handwriting: its aesthetics, its readability, its delayed arrival. Finally, Rembert Huser discusses the status of the signature and of filmic authorship in the film credits of Martin Scorsese.

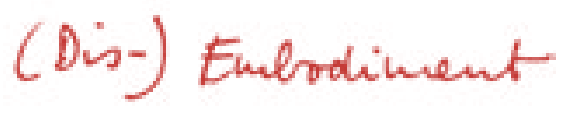

In the Bible, the prophet Daniel recounts a miraculous act of writing that functions as a divine warning signal on a wall in the palace of King Belshazzar. The passage from the Bible reads: 'There appeared the fingers of a human hand writing on the plaster of the palace wall' (Daniel 5, verses 5-6). The sign is magically written in indecipherable script, and the absence of a physical piece of writing comes to stand for the presence of God.7 This dialectical structure of absence and presence precisely pinpoints the great mystery of handwriting: that handwriting always gets its cultural authority from its claim of springing from a physical and living hand - a claim that Benjamin would call the undividable 'here and now' of presence. This corporeal dimension makes handwriting an absolutely individual and non-exchangeable sign, almost as unique as fingerprints or other biometrical data. And this holds even if the subject of writing is no longer there, as Derrida emphasizes, 'even after death' $(1988,5)$.

The concept of the hand and its role in the process of writing have been discussed in various academic disciplines. Pedagogy formulates rules to discipline the child's (right!) hand; philosophy connects the activity of the mind to the movement of the hand; and grapho-psychology uses the expression of the hand's movement to trace the writing subject's character. ${ }^{8}$ Technical writing tools, in these discourses, are often regarded as detaching script from hand, as 'disembodiment' of what has traditionally been seen as a highly corporeal act, implying a 'loss of self'. The assumptions made in these discourses come to stand in another light however, if handwriting is not redeemed by technical writing methods but, instead, itself appears in a second medium. How do such writing methods affect our understanding of an authentic physical body? This problem can be described by the example of Microsoft screensaver Sports, published in Windows 98. This screensaver produces white stripes that emerge from a dark, marbled background, forming zeros, crosses, and other symbols, written in real time by a hand writing with chalk on a blackboard or tablet. This screensaver generates handwriting, but just like the divine writing on the wall, the screensaver's writing is written as if by magic, urging us to ask who is writing here? Is there some artificial intelligence with a virtual body inside the computer? Post-post-modern paranoiacs may suspect some lonely cyborg sending cryptic messages. Or perhaps it concerns one of those creatures N. Katherine Hayles describes as those who 'reconcile themselves to living inside 
the computer', and who 'often create INTERFACES that allow them to preserve the illusion of ordinary human existence' (22). But how, then, are these interfaces to be conceived of? How can we as users with an 'ordinary human existence' interact with this bodiless subject of writing inside the screensaver, as if any intervention, any manipulation of the machine, might immediately erase the script from the screen, thus making the writer even more absent?? By simulating the physical inscription technologies of handwriting, the screensaver Sports emphatically claims the physical presence of an individual writing hand. Yet this presence then appears to be an effect of the user's absence. Conversely, one could say that the bodiless writer inside the screen produces this user only as an effect of its absence, reducing it so to speak to a physical leftover showing up only when the screensaver is put to rest (cf. Neef, 2004).

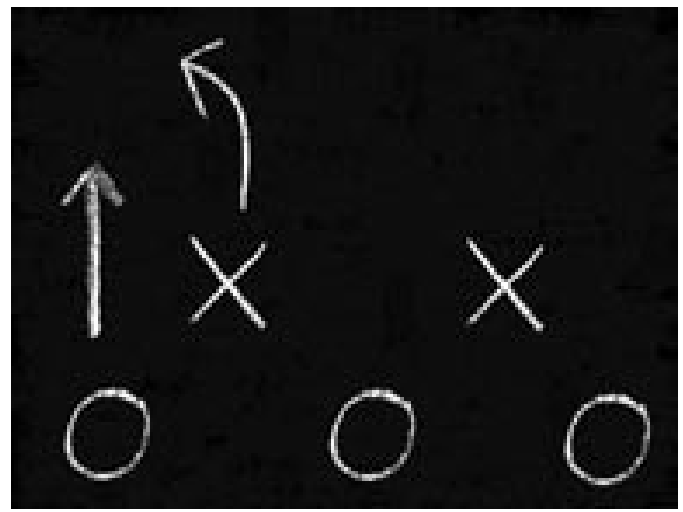

4. A screen capture from Microsoft screensaver Sports

The screensaver enacts handwriting both as the archi-writing of a hand that claims physical presence, authenticity, and uniqueness, and concurrently signifies the place of handwriting's disembodiment. As screen writing it does, indeed 'save' a hand, but not quite, because it simultaneously inscribes and suppresses the proliferation of manual writing; it embodies and disembodies at the same time.

The focus of this third section is to ask where the writing hand is to be located in a dialectics of presence and absence, man and machine, medium and hand, and how this dialectics is to be conceived of if handwriting is not immediately performed 'by hand' but in a second medium. Eric Ketelaar turns again to the archive when he asks for the ways in which writing on, in or with new archiving machines impresses the work of the archive, when the hand of handwriting has been transformed into a part of the machine. Thomas Fechner-Smarsly, then, concentrates on a form of handwriting in which the claim of corporeality takes shape most radically: the fingerprint. Fechner-Smarsly in his article relates the concept of hand- 
writing to two concepts closely related to it and yet distinctly different from it: those of 'trace' and 'imprint'. When remediated in contemporary art, the fingerprints produced in both ink and blood can only be read through the grammar imposed by genetic codes. Begüm Firat concentrates on medial enactments of Arabic writing, which - due to its flowing movement - strikingly resembles handwriting, even if typed on a keyboard or visualized on a computer screen. Firat discusses Shirin Neshat's famous photographic work in which Arabic handwriting is dominant. Finally, Sonja Neef investigates the cultural practice of tattooing as a writing practice that takes the living body for its medium, a body that appears absolutely in-exchangeable and singular. And yet, Neef argues that a tattoo can only become meaningful as writing when it takes a repeatable, or citational form, when its presence will function as a sign and do this also in a past and in a future present. The combination of hand and writing, thus considered, can no longer be considered a stable and unitary source of authenticity, singularity and originality, since it is driven by difference.

This book was funded by the Dutch Research Organization (NWO). A warm thanks is directed at Saskia Lourens and Bart Plantenga for their professional and friendly support in copy-editing this book.

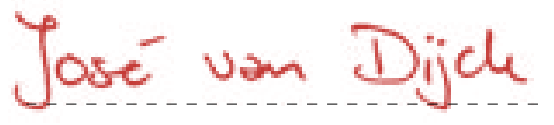

SIGN HERE!

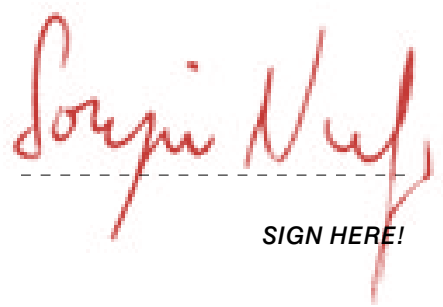


Notes

1. Thanks to Jan Simons, for having pointed out this particular symbol at the twoday conference ReMediating Handwriting, Weimar, June 18, 2004.

2. For a seminal study of the history of the signature see Fraenkel, La signature, 1992.

3. Arguing within a Lacanian frame, for Kittler, film refers to the realm of the imaginable, the gramophone with its emphasis on the presence of the voice refers to the realistic, and the typewriter is bound up with the symbolic system of language.

4. Goodman elaborates on the difference between allographie and autography in chapter 3 'Art and Authenticity'.

5. The topos of the hand in the work of Heidegger is studied in detail by Jacques Derrida in La main de Heidegger.

6. For detailed discussions of digital enactments of the handwriting of Leonardo as screensaver and as digital codex on CD-ROM, we refer to Neef 'The
W/Ri(gh)ting Hand. Leonardo da Vinci as Screensaver', respectively 'Die (rechte) Schrift und die (linke) Hand'.

7. For a detailed analysis of this magic act of writing, we refer to Neef 2000 , 64-68.

8. For a history of handwriting education from Victorian writing culture until automatic writing in the 20th century, see Plakins Thornton. An overview of the philosophical question of mind and hand from Quintilianus until Renaissance is given in Zwijnenberg, 65-82. For graphology as a modern science in the humanities we refer to the work of Ludwig Klages (1905-1927). 9. This is what Hayler would call a 'technotext', texts which 'interrogate the inscription technology that produces it, it mobilizes reflexive loops between its imaginative world and the material apparatus embodying that creation as a physical presence' (25).

\section{Works Cited}

Benjamin, Walter. 'The Work of Art in the Age of Mechanical Reproduction', in: Hannah Arendt (ed.), Illuminations. Trans. Harry Zohn, 217-251. New York: Schocken, 1969 (1936).

Bolter, Jay David, and Richard Grusin. Remediation. Understanding New Media. Cambridge, MA and London: MIT Press, 2000.

Derrida, Jacques. 'Geschlecht: différence sexuelle, différence ontologique. La main de Heidegger (Geschlecht II)', in: Psyché. Inventions de l'autre. Paris: Galilée, 1987.

Derrida, Jacques. 'Signature Event Context', in: Limited Inc., 1-21. Evanston: Northwestern University Press, 1988.

Fraenkel, Béatrice. La signature. Genèse d'un signe. Paris: Gallimard, 1992.

Goodman, Nelson. Languages of Art: An Approach to a Theory of Symbols. Indianapolis: Hackett Publishing Co., 1976.

Hayles, Katherine N. Writing Machines. Cambridge and London: MIT Press, 2002.

Heidegger, Martin. 'Parmenides. Freiburger Vorlesung im Wintersemester 1942/43', in: Manfred S. Frings (ed.), Heidegger Gesamtausgabe. II. Abteilung: Vorlesungen 1923-1944, Band 54, Frankfurt am Main: Klostermann, 1992 (1942/43).

Hörisch, Jochen. Ende der Vorstellung - Die Poesie der Medien. Frankfurt am Main: Suhrkamp, 1999.

Kittler, Friedrich. Grammophon, Film, Typewriter. Berlin: Brinkmann \& Bose, 1986.

Klages, Ludwig. Frauchiger, Funke, Groffmann, Heiss, and Schröder (eds.), Graphologie I. Bonn: Bouvier, 1968.

Neef, Sonja. Kalligramme. Zur Medialität einer Schrift. Anhand von Paul van Ostaijens 'De feesten van angst en pijn'. Ph.D. Diss., Amsterdam: Asca Press, 2000.

- 'The W/Ri(gh)ting Hand. Leonardo da Vinci as Screensaver', in: Nancy Pedri (ed.), Travelling Concepts III: Memory, Image, Narrative, 341-355. Amsterdam: AsCA Press, 2003. 
- 'Die (rechte) Schrift und die (linke) Hand', Kodikas/Code. Ars Semeiotica. 25, no. 3-4, 157-174. Tübingen: Günter Narr, 2003.

- 'Handwriting and Screensaving. On Microsoft Screensaver Sports', lecture on July 18, 2004 at the symposium on 'ReMediation', Bauhaus Universität Weimar, 2004.

O’Toole, James M. 'On the Idea of Uniqueness', in: American Archivist 57 (Fall 1994), 632-688, 1994.

Plakins, Thornton. Handwriting in America: A Cultural History. New Haven: Yale University Press, 1996.

Zwijnenberg, Robert. The Writings and Drawings of Leonardo Da Vinci: Order and Chaos in Early Modern Thought. Cambridge: University Press, 1999. 

Sign Here!

Section One:
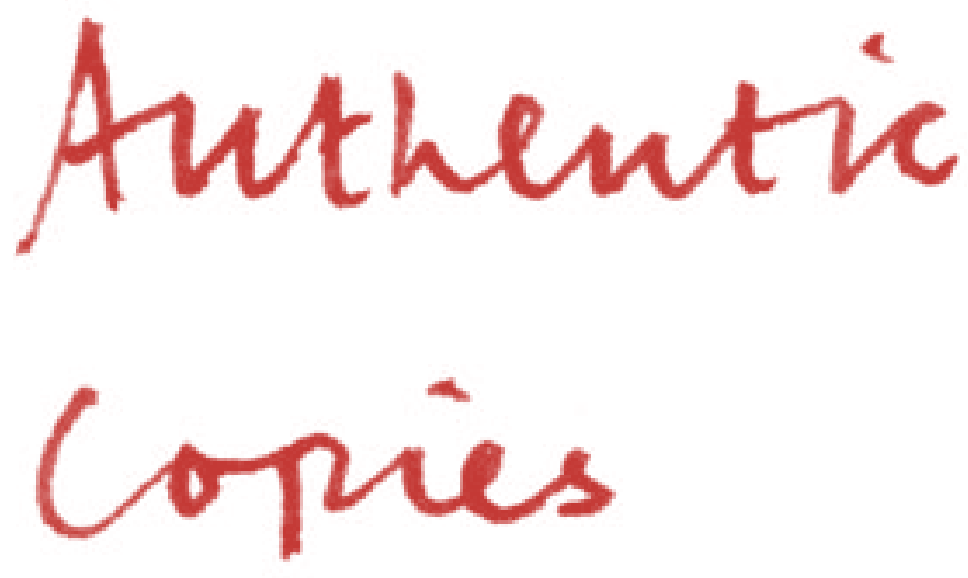



\section{Authentic Events:}
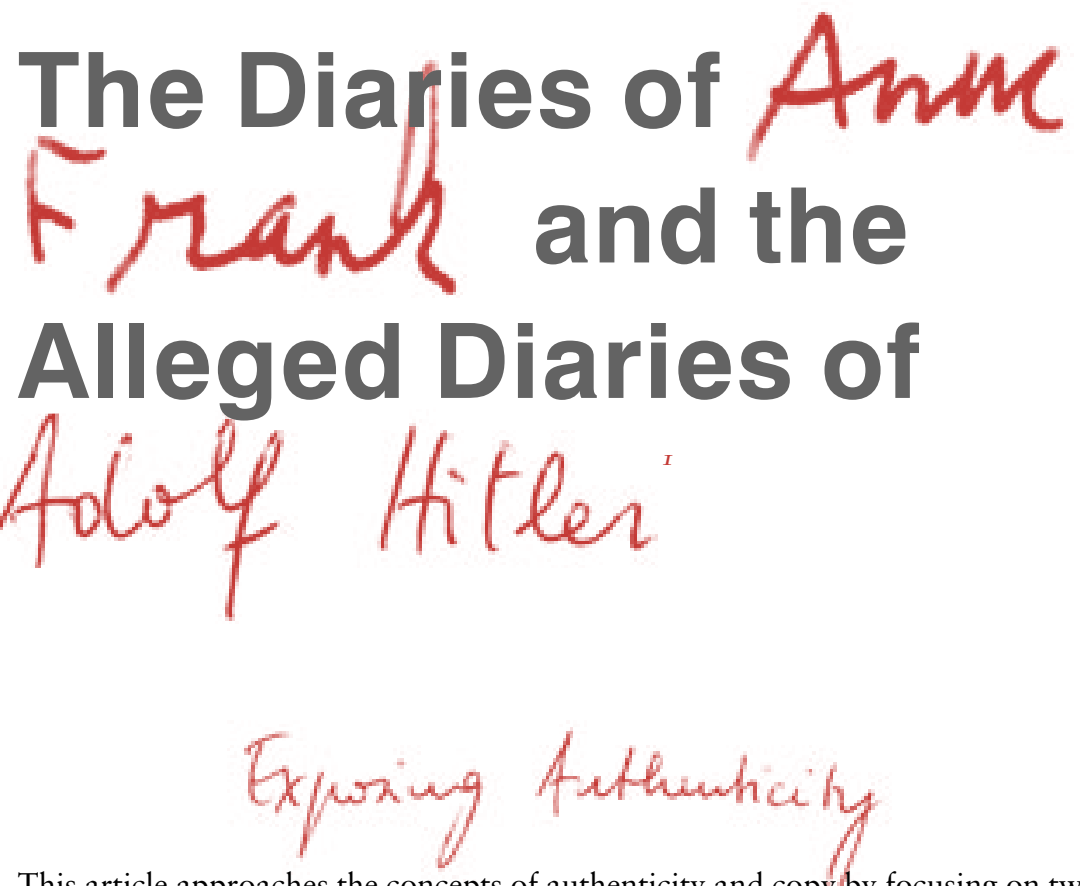

This article approaches the concepts of authenticity and copy by focusing on two autographs whose authenticity is at the core of a longstanding and culturally urgent debate: Anne Frank's diaries and the alleged diaries of Adolf Hitler. Combining these cases by discussing them in one and the same paper may, at first sight, seem odd, or even blasphemous. By taking a closer look at these diaries, however, I will concentrate on a rhetoric of authenticity that they share and that, at the same time, distinguishes them radically. The aim, then, is to arrive at a differentiated view of the concept of the authenticity of handwriting and of the cultural significance of the idea of the original. Both diaries were subject to a series of investigations into their authenticity. For Frank, several attempts were made to prove that the diaries were false and - along with this - that the Holocaust was a lie. For Hitler, in contrast, the aim was to prove that the diaries were authentic, and - along with this - to fill in the flatness of der Führer's public persona with the private depth of an original, personal character. Both diaries had a serious impact on how we deal with the past and on how we locate this past in our present culture between memory and amnesia.

Both for the diaries of Frank and for the alleged diaries of Hitler, the discourse of authenticity is to a large extent based on archival work. The primary scene for studying the authenticity or falseness of these manuscripts, then, is the archive, understood as a location where authenticity is generated by means of certification and custody. ${ }^{2}$ However, 'exposing authenticity' does not only mean exposing a 
document as true or false, one of the basic operations of the archive, but it can also refer to the act of exposing objects in a museum, literally understood as placing the object in a showcase, lighting it, and equipping it with an explanatory sign. The goal of this paper is to explore both 'loci' of authenticity: firstly, the archei on as an official hall that guarantees authenticity through the very architecture of an institutionalized authority, and secondly, the müsēum - as a location where objects are displayed or 'exposed' in such a way that authorizes their function as authentic witnesses to the past, as semiophores in the sense of Krystzof Pomian (1990, 30): visible objects that gain their meaning by referring to the hidden, the invisible.

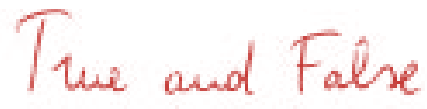

The diary of Anne Frank was first published in 1947 by Anne's father, Otto Frank, and was titled Het Achterhuis (The Secret Annex). Since then, it has been translated and published in more than 60 languages, and has become one of the most widely read books in the world. However, from the 1950s on, the authenticity of the diary was repeatedly questioned by several ex-Nazis and neo-Nazis in a variety of pamphlets, brochures, and other publications that received extensive media coverage. Their accusations were all aimed in the same direction, claiming - to quote from a pamphlet distributed by Schönborn - the diary of Anne Frank to be a 'forgery and the product of Jewish anti-German atrocity propaganda to support the lie of six million gassed Jews' (quoted from Da Silva). Between 1960 and 1993, these allegations became the subject of five lawsuits in Germany, mostly filed by Otto Frank, sometimes with the Anne Frank House as co-plaintiff. ${ }^{3}$ In all these cases, the verdict that the accused were guilty of slander and publicly inciting racial hatred was grounded in evidence that the diary was authentic.

Like the diary of Anne Frank, the alleged diaries of Adolf Hitler and the debate of their authenticity received extensive media coverage. Their discovery created a major stir in the media. On May 5,1983, Germany's best-selling weekly magazine Stern headlined the sensational find of secret diaries written by Adolf Hitler. ${ }^{4}$ The discovery of these documents was presented as an adventurous story about an heroic pilot named Friedrich Gundlfinger who escaped Berlin at the moment of Russian liberation in May 1945, carrying with him a safe containing secret documents of der Führer, among which were his personal notebooks. Almost forty years later, Gerd Heidemann, a Stern reporter, claimed that these diaries had shown up again. He explained that he had received the notebooks from an intermediary who had smuggled them into the country from East Germany by intervention of the Institut für Staatssicherheit (East German Secret Service) and some dubious generals in the Nationale Volksarmee (East German Army) (Heidemann in Stern, April 28, 1983). In fact, Hitler's notebooks, along with other Nazi memorabilia, were manufactured by Heidemann's mysterious intermediary - Konrad Kujau, alias Dr. Fischer, a forger and dealer in Nazi relics who found his business partners in collectors obsessed with the Third Reich. He had also faked, for example, handwritten manuscripts of Mein Kampf and paintings 'by Hitler'. Almost a quarter of the more than 
700 artworks represented in Billy Price's Adolf Hitler: The Unknown Artist (1983) were actually forgeries by Kujau. 5

Both the diaries of Anne Frank and the alleged diaries of Adolf Hitler have been investigated by a battery of internationally acknowledged handwriting experts. In what follows, I will not provide a complete overview of the jumble of certifications and reports. Rather, I will ask how authenticity and falseness are conceived of in these assessments by analyzing some snapshots, each showing a fragmented episode from a long and arduous quest for truth. These snapshots are taken at the two locations central to this article: first, the archive, and later in this article, the museum.

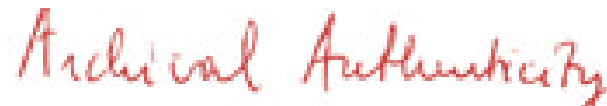

The archival investigation of a document's authenticity can roughly be divided into three types of approaches. First, from an historical point of view, a text-critical analysis questions the text's content for grammatical, logical, and historical consistency and places it in a context. Second, the material authenticity of a document is investigated on the grounds of chemical tests. And third, handwriting experts analyze the visual performance of the actual writing.

Initially, it was the first procedure - the historical approach - that, for Hitler's notebooks, led to a certification of authenticity. ${ }^{6}$ Since Kujau had collected and read an extensive personal library about Hitler, historians specialized in the history of the Third Reich could not immediately expose the manuscripts as forgeries. Only upon a second viewing did a team of archivists from the German Bundesarchiv locate Kujau's sources: Max Domarus' well-known publication of Hitler's speeches and proclamations, Gerd Rühle's Das Dritte Reich, as well as the Nazi daily Der Völkische Beobachter. They identified a series of identical mistakes concerning dates, names of institutions, and historical facts made both by Kujau and one of his sources (Henke, 310-314).

The initial certification of authenticity was also based on the immense amount of autographical material, which included a collection of 62 volumes of diaries, accompanied by a massive archive of 300 watercolor paintings, drafts for speeches and letters - all 'by Der Führer', and, last but not least, his World War I uniform and helmet (Hamilton, 1). Hugh Trevor-Roper evaluated the find in the London Times as follows: 'whereas signatures, single documents, or even groups of documents can be skillfully forged, a whole coherent archive covering 35 years is far less easily manufactured... The archive coheres as a whole and the diaries are an integral part of it'. ${ }^{7}$

But there were also skeptical voices, among which the famous historian David Irving and the German Bundeskriminalamt. Their critique introduced a second phase in which the Bundesanstalt für Materialforschung und -prüfung (German Criminal Court Laboratory), by chemical analyses, identified synthetic fibers in the binding as well as paper-bleaching agents in the paper of some volumes, neither of which were used before $1945 .{ }^{8}$ Whereas the historical investigation of the al- 
1

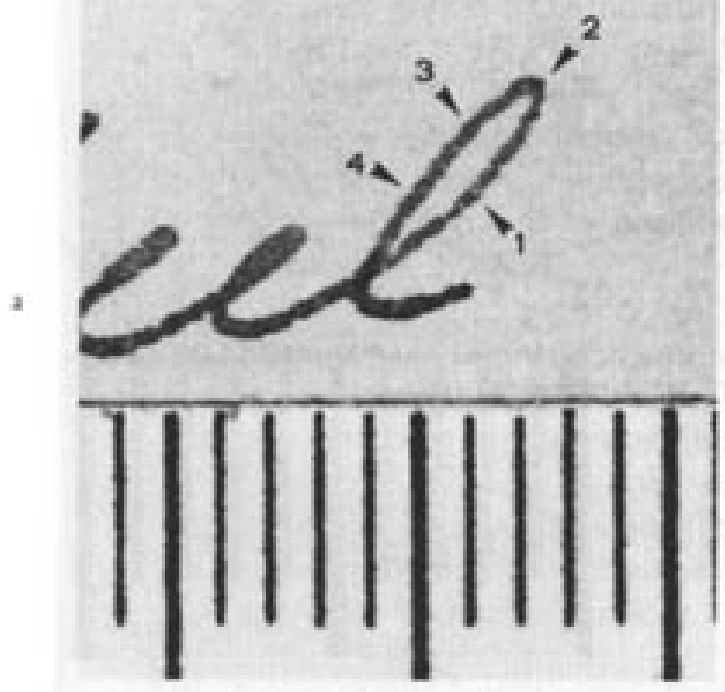

t

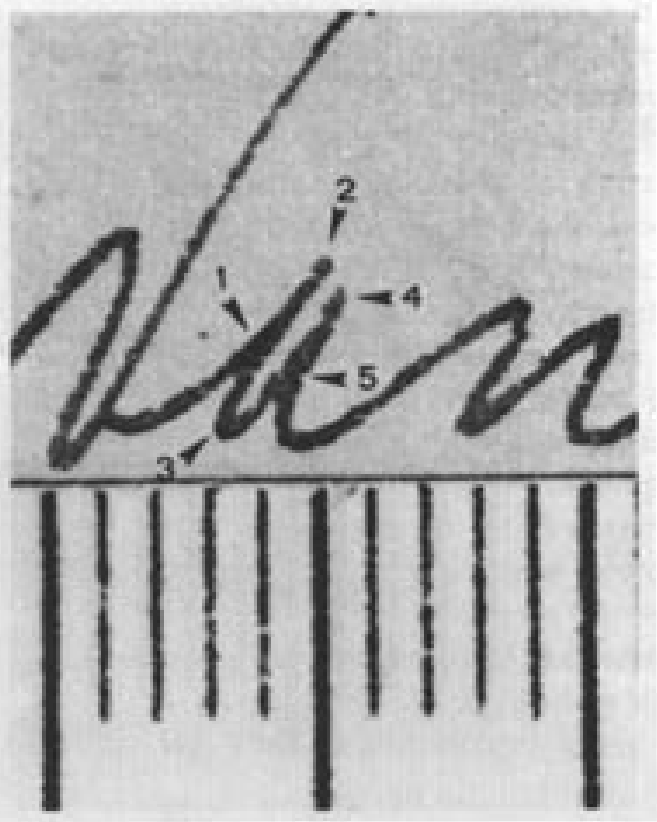

2

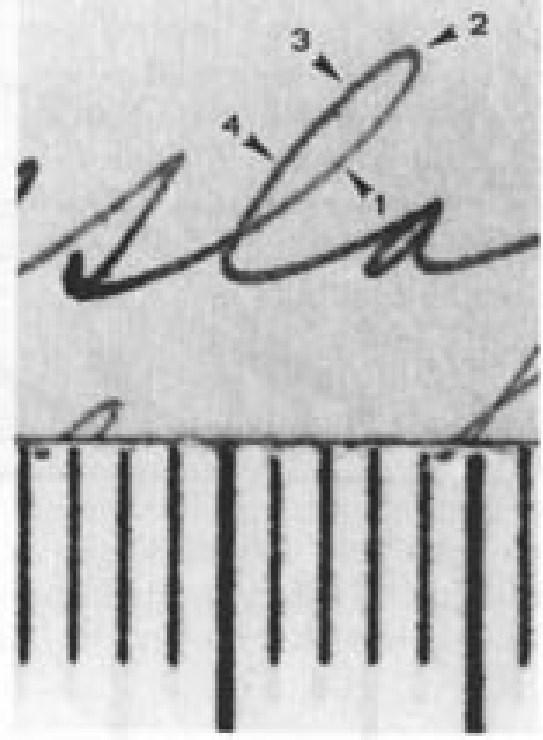

2

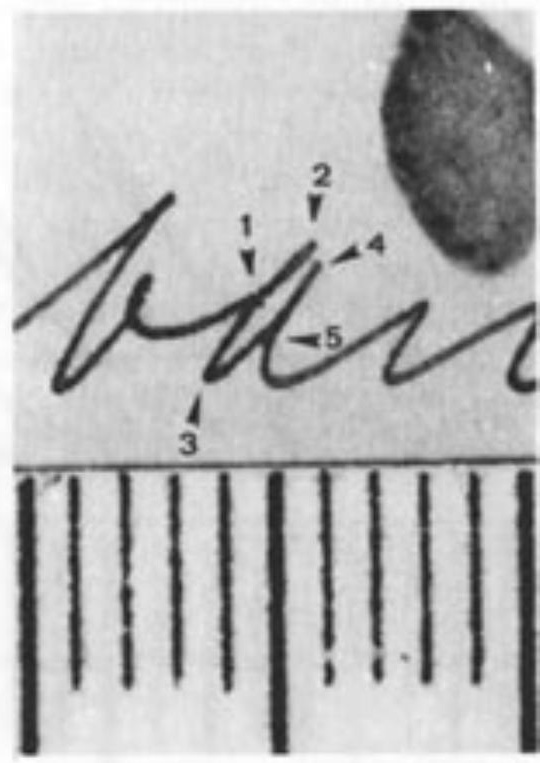

1. Extract from Hardy's summary of the report of the Gerechtelijk Laboratorium in Rijkswijk/ Netherlands (150-151) showing 'micro-characteristics' studied as visual 'points of identification' in the handwriting of Anne Frank. The examples on the left are from reference material, those on the right from the first diary. 


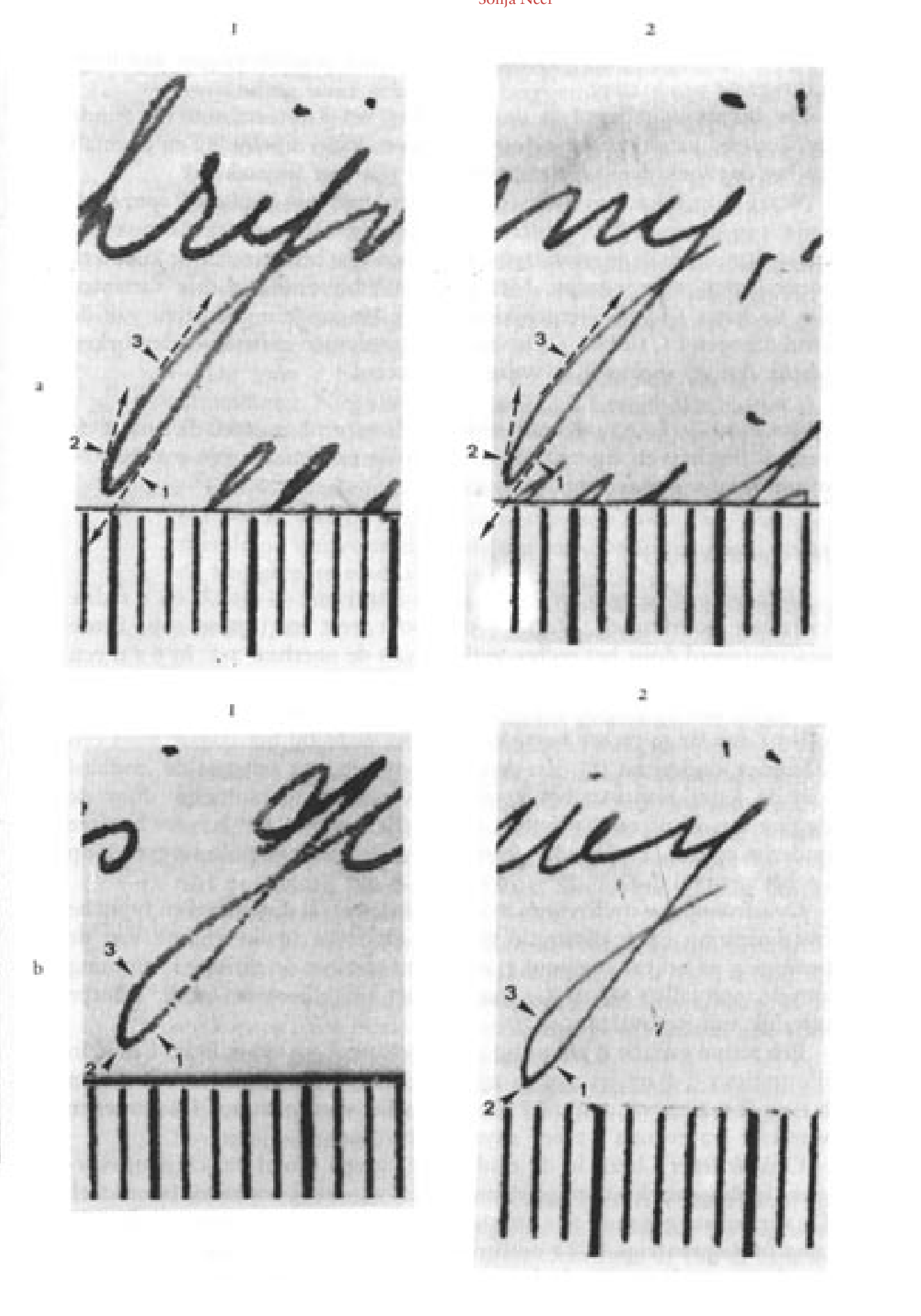


leged Hitler diaries had initially failed, this chemical investigation provided positive evidence that the diaries were fake.

To achieve positive evidence of a document's falseness, historical and technical investigations are undoubtedly effective. To prove the authenticity of an authentic manuscript, however, the evidential value of these methods is limited, since they can only demonstrate a manuscript's falseness. This is what happened in the assessments of the diaries of Anne Frank in which no anachronisms could be found - neither historical nor chemical. 9 To achieve positive evidence of authenticity, the Gerechtelijk Laboratorium (Dutch Forensic Laboratory) investigated the specificities of the handwriting and categorized them in an extensive list of so-called 'micro-characteristics' (microkenmerken) of both the writing movement and the writing pressure. ${ }^{\text {Io }}$ These micro-characteristics were studied as visual 'points of identification' (identificatiepunten) appearing in the extensions of a character (Illustration 1). If they follow a systematic scheme they can be compared with certified reference materials, in this case, with Anne Frank's letters and school albums. This comparative method is based on the idea of the mathematical iteration of differences and resemblances. The result of such an investigation is statistical in nature and is, therefore, expressed in terms of a degree of probability. For Anne Frank's diaries, the result was phrased very carefully as 'probable to a degree bordering on certainty that the diary originates from the producer of the reference writing, Anne Frank'. ${ }^{I I}$

These reports offer ample material for a critical discussion of the concepts of authenticity, copy, and their relation to the ideas of originality and uniqueness. First, it is striking that the chemical analysis can only be performed on an original document, suggesting that a unique 'original' cannot be replaced by a copy without compromising the manuscript's authenticity. Unlike chemical analysis, both historical analysis and handwriting assessments can be performed on a high quality Xerox copy. These methods rely on the idea of writing as iteration, the idea that a written text, like a musical symphony, is repeatable, spellable, or, to echo Nelson Goodman, 'allographic' (Goodman, chapter 3). Historical assessment, which concentrates on a document's content and on the coherence of an archival collection relies precisely on this idea of writing as allography, that is, a writing of which the authenticity is not compromised when the text is copied or re-printed. A manuscript's physical materiality, in this view, appears as a nearly invisible medium. Strictly speaking, the document does not even have to be seen; having the written text read aloud is enough. This 'blindness', in a way, pertains even more to the chemist, who - despite his emphasis on the material original - entirely neglects a document's content. Both the chemist and the text-historical analyst ignore the visual performance of actual specific handwriting. They are either only interested in a manuscript's material originality and singularity, or, conversely, in the iterability of writing as allography. 


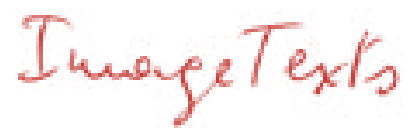

Handwriting, I will argue, opens up a third space between originality and copy, between singularity and iterability. On the one hand, handwriting originates in the physical presence of an individual and corporeal writer who, by a movement of the hand, generates a line, a 'trace', providing it with an un-exchangeable visual quality. The first test a handwriting expert performs when investigating a piece of handwriting is to turn the manuscript upside-down, looking at it as a pure visual 'image' without noticing its alphanumeric dimension as 'writing'. Thus, handwriting, like painting, operates as 'autography' in Goodman's sense, since it does not allow for repetition without being considered a forgery.

On the other hand, handwriting is still bound up with the symbolic system of language; it is still writing and, because the central principle of writing is repetition, indeed allows for copying. Entire libraries were comprised of handwritten transcripts. The archive, however, unlike the library, insists on the originality of a document. It preserves unique records that cannot be found anywhere else (O’Toole, 633). Archival preservation of a manuscript cannot be achieved by a typewriter, but only by those technologies that reproduce its image-quality: Xerox copies or microfiches. Thus viewed, handwriting is both allography and autography, writing and image, what could be called an 'ImageText'. Like Derrida's signature, handwritten ImageText is both unique and repeatable, singular and iterable. Its cultural importance in our contemporary world of print and digital media resides precisely in this paradoxical structure: iterable singularity, singular iterability (Derrida 1988).
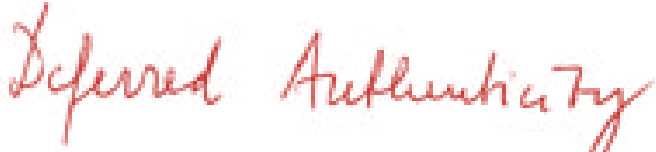

The assessments of Anne Frank's diaries and of the alleged notebooks of Adolf Hitler invite yet another discussion about the relationship between authenticity, singularity, and originality. These assessments show that authenticity cannot be proven on the grounds of a single document because each document is always embedded in an encompassing system of documents constituting the archive. For Frank's diaries, their authenticity was explored by comparing them with documents of which the authenticity was certified. A document's authenticity is thus not a quality in its own right, but is generated by a system, a body of sources, whose authenticity is guaranteed by the archive's unity, homogeneity, and consistency. This system is simultaneously both certifying and certified. The concept of authenticity here takes the shape of a trace, as it cannot be located in a unitary origin; it is, as Derrida would say, always already deferred. ${ }^{I 2}$

For Anne Frank's diaries, the German Criminal Court Laboratory recognized this problem. Hence, for the investigation of the reference material the experts followed the same technical procedures they had used for the diary itself, including examinations of stamps, postmarks and censure seals on the envelopes of Anne's letters. And yet, the evidence that the reference material was authentic was finally 
based - as with the diary itself - on the fact that no anachronistic materials or document elements could be found (Hardy, 125, 133, 139). For the alleged diaries of Hitler, there was only little certified reference material available in the Bundesarchiv. And so it happened - according to Stern reporter Bissinger - that Gerd Heidemann compiled the so-called 'dossier Heidemann', a file containing samples of autographs by Hitler to be given to handwriting experts as reference material. 'This... file', Bissinger writes, 'had just one minor flaw: big parts of it stemmed from the same workshop in which the Hitler diaries were also produced. Thus the experts... found that, indeed, these writings stem from the same pen' (173). ${ }^{13}$

Comparing counterfeit with counterfeit here turns out to be the equivalent of comparing original with original. Kujau's diaries were indeed authentic, and they were originals - authentic and original forgeries. In addition, the documents offered by the 'dossier Heidemann' were partly authenticated by certificates, which were, in turn, manufactured by Kujau. Whatever we come up with, the idea of authenticity can no longer be rooted in a single and indivisible origin, for any certificate of authenticity is as much in need of authentication as the doubted document itself. For Hitler's notebooks, it was precisely the denial of this movement of deferral that led to the initial authentication of the notebooks. Authenticity, with respect to handwriting, can neither be definitively cut off from the concepts of originality and singularity, nor can it be comfortably filed under the idea of an imperturbable originality or a handwriting's singular occurrence in the world.
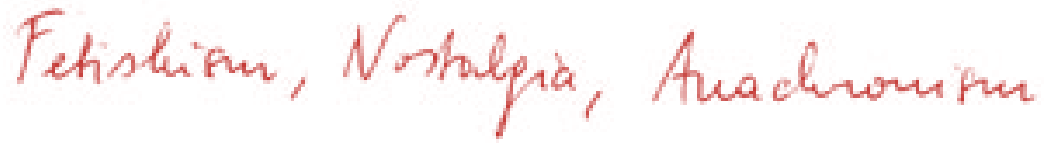

And yet we do desire originals. We desire them so profoundly that the Anne Frank Stichting had to produce two facsimile replications of all six notebooks of Anne Frank. These copies serve to protect the original from damage caused by crowds of scholars and film crews, but also to preserve the 'aura' of the 'original' notebooks for future generations. Due to digital technologies of scanning, analyzing, and reproducing documents, these copies look perfectly authentic. ${ }^{I 4}$ Each of them is fitted with the same tiny details that make the original so distinctive: the red-andwhite cloth binding of the famous autograph album; the photographs, picture cards, pieces of paper, as well as letters and postmarked stamps on envelopes glued inside the book; and the clasp lacking the small metal end that fits into the locking mechanism, yes, even the rust spots on that clasp (Tanja). ${ }^{\text {IS }}$ The more we desire the original, the more counterfeits we get. ${ }^{I 6}$

We desire the original so much that Stern at the time was willing to pay the princely sum of 18.5 million German marks to gain possession of Hitler's diaries. And the bill got paid, because even though this sensational story turned out to be a canard, the magazine's profits increased to 190 million German marks that year (Bissinger, 227). One can hardly avoid the association with the Marxist idea of commodity fetishism here, that is, the idea that in capitalist society a commodity is alienated from its specific qualities and deprived of its actual value. Instead, a commodity is perceived only for its exchange value. ${ }^{I 7}$ For the Hitler diaries, their cul- 
tural role as objects of economic exchange cannot be overlooked. The more money Stern spent, the more convinced they became of the diaries' authenticity. The immense money value was justified by Stern claiming that these diaries had a substantial historical value, necessitating a revision of the history of the Nazi era. The archivists performing the historical authentication were, on the contrary, astonished by the banality of the diaries' historical content, which was reduced to shallow descriptions of public events lacking any private, political, or conceptual reflection (Henke, 309-310).

The value of these diaries as objects of economic exchange did not primarily result from their historical value but, I will argue, from their autographic dimension as handwriting, from - to echo Benjamin - their 'auratic' character, their physical materiality which claims an original and singular 'here and now' that can be conceived of as an indivisible origin which has the potential to unify past and present. This nostalgic desire for auratic handwriting follows the logic of the fetish in still other than economic terms. In psychoanalytic discourse, the fetish is understood as an object that one feels strongly attached to. Freud, in his essay on fetishism (1927), describes the fetish as a substitute for the mother's absent penis. The child believes that the mother's penis was once there but that she was deprived of it. The insight that the mother does not in fact possess a penis leads to a metonymic displacement of the absent penis onto some other part of the body or its continuation, such as a piece of velvet, jewelry, or - as in the Kujau-case - a Word War I uniform and helmet. This logic of replacing a desired yet unavailable object with another object, I will argue, also applies to handwriting. For our cultural desire for the 'auratic' original derives from a (childish) belief in an original and physical presence, embodied in a writing movement conducted by a writing hand which is indivisible from the subject of writing and yet has been deprived of it. But not without leaving a trace in the form of a 'trait' that becomes a metonymic substitute for the writer in a future present.

It is significant that Hitler's diaries were part of an encompassing collection of Nazi relics circulating among dealers and collectors, including not only documents and paintings, but also flags, uniforms and helmets, swords, daggers, and pistols. Moreover - and this seems striking for my argument - Kujau had discovered that his customers were 'always impressed by seals, especially wax seals over silk ribbons' (Hamilton, p. 12). The historical value of these objects seems doubtful. Instead, they are pure fetish; literally the product of a perversion that can be specified as a collecting instinct. This fetishist attitude of collecting is, in psychoanalytical terms, predicated on a separation between subject and object which - and I am following Mieke Bal's theory of collecting here:

makes for an incurable loneliness that, in turn, impels the subject to gather things, in order to surround him- or herself with a subject-domain that is notother. Small children do this, collecting gravel, sticks, the odd pieces that grown-ups call junk but which, for the child, has no quality other than constituting an extension of the self, called for to remedy the sense of being cut-off. 
It is precisely this experience of being separated from a wholeness incorporated by the far-away and long-ago homeland of the Third Reich that slips into a present here and now, that made Hitler's alleged diaries so successful. Thus seen, the Hitler diaries cannot be understood except as a fetish that is the result of both an economic and historical desire that finds its specific shapes in Heidemann's obsession with the Third Reich; in Stern's attachment to a commodity alienated from its empty historical value; and in a global public awaiting the resurrection of either a monster or a messiah.

For the Frank diaries, the concept of fetishism raises a few uncomfortable problems. For one thing, it suggests that one must, paradoxically enough, assume that a similar experience of deep and fundamental lack forms the motive for almost one million people a year to visit the Anne Frank House in Amsterdam as an authentic place where the diaries were written and can now be seen. What, one must ask, distinguishes the ardent desire of the visitors of the Anne Frank house in their search for authenticity from the fetishist desire of the recipients of the alleged Hitler diaries? Is Anne's diary to be considered a fetish, as the Hitler diary is? There is a subtle difference, however - there must be one. But how can we understand this difference? Can it be attributed to the ultimate distinction that classifies Anne Frank's diaries as true and Adolf Hitler's diaries as false? Is it enough to point to the fact that Frank's diaries were not written on demand, as Hitler's diaries were? Rather, they were given to us by Otto Frank when he came back from the extermination camp in Auschwitz and forced upon us as an inconvenient guest. But if they came without invitation, how, then, could they become a monument of shared history, if not by inviting a past to speak back to, and intermingle with, our present? How is this anachronism to be viewed, this conflation of past and present that is put forward by the principle of iteration, of repeating - in a paradoxical gesture - the singular original? And how is it related to the nostalgic desire for Nazi relics rooted in fetishism?

I have thus far located these questions in terms of the archive, with its practices of certifying original and singular records of the past and preserving them for the future, but let us now turn to that other locus of cultural memory: the museum. Like the archive, the museum's capacity for 'exposing' a document as true or false is grounded in the authoritative power of the institution. The archive produces authenticity within the inside of a more or less closed official hall - and this shield against the public is an integral part of archival legacy. Archival semiophores do not only refer to the invisible, but they are also often stored invisibly in the stacks, while the museum's rhetoric is unbridgeably grounded in this very act of 'making

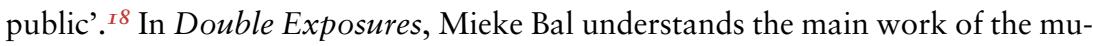
seum as a posture or gesture of exposition:

Exposing an agent, or subject, puts 'things' on display, which creates a subject/object dichotomy. This dichotomy enables the subject to make a statement about the object. The object is there to substantiate the statement. It is put there within a frame that enables the statement to come across. There is an addressee for the statement, the visitor, viewer, or reader. The discourse 
surrounding the exposition, or, more precisely, the discourse that is the exposition, is 'constative': informative and affirmative.... In expositions, a 'first person', the exposer, tells 'a second person', the visitor, about a 'third person', the object on display, who does not participate in the conversation. But unlike many other constative speech acts, the object, although mute, is present. (Bal 1996, 3-4)

In what follows, I will explore the expository strategies of the museum within the theoretical framework offered by Bal. Concretely, I will continue my examination of the concept of authenticity in the Anne Frank Museum in Amsterdam, asking how the museum, as a cultural agent and as a first person in the discourse on the Holocaust, presents the diary of Anne Frank as an exhibit, a third person to be looked at as a public object. I will investigate the discourses proposed by Bal, that is, the museum's statement on the Holocaust as an authentic and representable historical event, and Anne Frank's voice as the object on display. The aim of my analysis is to mobilize the fixed positions of authoritative speech, thus allowing Anne Frank to 'speak back' - both to the museum's documentary discourse and to us, the visitors, the second persons of this speech act.

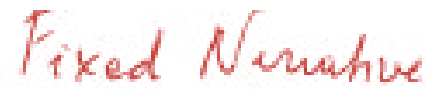

December 2002, a cold and rainy day in Amsterdam. I join the queue of people waiting at the foot of the Westertoren. Freezing and wet, we stand in line for two hours to get into the Anne Frank House. Nobody complains or is impatient. What does our suffering mean, after all, compared to what she went through? Once inside the house on the Prinsengracht, we follow the prescribed route, leading us from the front part of the house via a landing with a movable bookcase to the back part. There is no escape; we climb the same stairway she once climbed to the secret annex to go into, hiding for 25 months until the arrest.

Once past the bookcase it becomes silent in the canal-side house. This is exactly the situation described by Jessica Durlacher in the opening of her novel The Daughter, which tells the story of Max and Suzanne and their second generation Holocaust trauma. ${ }^{I 9}$ 'It is almost a funeral', Durlacher writes. Visitors shuffle through the museum silently or quietly whispering, as if they were observing the requirements for surviving once observed by the people in hiding. 'We have to whisper, and tread lightly during the day, otherwise the people in the warehouse might hear us', reads a quote from Anne Frank's diary printed on the museum's wall.

I step into the small room once occupied by Anne Frank and Fritz Pfeffer. It is dusky in here because the windows are covered with curtains like they were at that time to protect the inhabitants from outside gazes. There are no furnishings here; the entire annex was cleared out by order of the Nazi occupiers. Since then - one can read in the museum's pamphlet - it has remained in its authentic state: authentic darkness, authentic silence, authentic emptiness. 
One of Anne's handwritten letters is exhibited in a display case in Anne's room. The darkness prevents the viewer from deciphering its contents, but there is a transparent accompanying label on the display case. I go nearer and try to read the inscription. However, on approaching the case, my own shadow darkens the letter. I want to try another angle, but I cannot because I have to share this room with others - just as Anne did with Fritz Pfeffer. From my new position, the muted light of an exposition spotlight reflects off the glass in a way that blinds me. I finally manage to read the sign that describes the manuscript as a 'farewell letter written by Fritz Pfeffer to his fiancée Charlotte Kaletta'. The letter itself remains unreadable, though. The feelings of love expressed in this letter remain undecipherable, and its beloved addressee Charlotte Kaletta is ultimately lost.

My tour through the museum leads me to the attic, a large, high and brightly illuminated room. Coming out of the dark secret annex, the visitors blink against the dazzlingly bright light that shines on glossy images documenting the Holocaust and on screens showing videotapes of Westerbork, Bergen-Belsen, and AuschwitzBirkenau. Only after the visitors have passed through this room do they arrive in the adjacent room where the diaries of Anne Frank are displayed in brightly-lit showcases.

Whereas Pfeffer's love letter was displayed vertically on the museum wall - like an image - Anne's notebooks are displayed horizontally, requiring the visitor not only 'to look at', but also 'read' them as text. The museum's topography, moreover, instructs me how to read them, as it shapes the walk through the museum as a narrative with a mono-linear and fixed structure, told by the exhibitor, revealed to us, whom Bal calls the museum's second persons. This story has a preface in the warehouse on the ground floor, its beginning is located on a dark platform with a movable bookcase, it continues with inescapable linearity up a stairway, and ends with the attic documenting the inhabitants' final destinations after they were betrayed. The museum's topography thus metaphorically instructs us how to read Anne Frank's diary: retrospectively. ${ }^{20}$ Any event reported in her diaries is supplemented by a meaning framed within a second history located outside - or literally: on top of - the actual annex: in its attic. We fill in the museum's empty rooms with our knowledge of what is not told in the diary: the arrest, the deportation, the camp. We look back at Anne's 13th birthday party when she was given her first notebook, and we frame this moment within the hermeneutic circle by pasting Anne's future into her past, thus creating a unitary history in which Anne threatens to become objectified into the always already victim of Nazi murder - which she was, indeed. But wasn't she so much more than that?

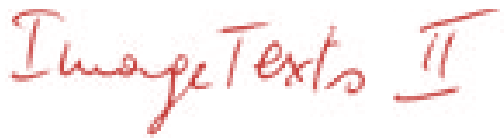

Like Anne Frank's diary, Fritz Pfeffer's farewell letter is displayed in a showcase, but does not seem meant to be read. Its presence there is grounded in a kind of 'cult value' as Benjamin ascribes to 'certain Madonnas [that] remain covered nearly all year round' (225). Their value, Benjamin states, resides in their being there, not in 
their being looked at. The expository gesture of Pfeffer's showcase in the museum is similar to this. As a 'speaking witness' to the past, Pfeffer's handwriting is invisible, or mute. Its value as a museum object, as a third person, does not primarily result from a readability that would give voice to the past. Rather, the letter only expresses material authenticity, physical presence, pure 'cult'. And yet, I would argue, this 'cult' character does not necessarily qualify as fetishism, as the Hitler diaries did in their alienation from historical discursivity. Why not?

The point is that Holocaust reports, if authentic, often risk being unreadable. Conversely, if they speak out the unspeakable and make their reading too easy, they risk being considered inauthentic. They are unreadable to me, a 'second person' visiting the Anne Frank House as a tourist in a foreign country where a language is spoken that I do not understand. And they were just as much unwritable for the inhabitants of this long-ago time who were hiding in the dark. Traumatic Holocaust experiences are difficult to incorporate into narrative memories, for narratives of memory require that the narrator structure events in time in order to become the 'master' of history rather than the object suffering the traumatizing events. ${ }^{2 I}$

Earlier in this essay, I considered handwriting to be an ImageText, and I will now focus on another analytic dimension this concept offers. W.J.T. Mitchell, in Picture Theory, relates the double structure of his notion of 'imagetext' to the concept of memory as both textual and pictorial. ${ }^{22}$ Text, for Mitchell, has to do with storytelling 'in the sense of a temporal sequence of events' (194). Image, on the other hand, has to do with imagery and imagination, which unfolds in space rather than in time. Whereas an image contemplates a private memory located in a region of the unknown, narrative seems to be a mode of knowing and structuring, of making memory writable and readable as public recollection.

For that reason, traumatic experiences often remain within the realm of visuality and cannot become narratives. At best, they become silent testimonies, like this drawing by Armando (Illustration 2), that can be understood as an attempt to write. Yet, as an ImageText, it is cut off from its alphanumeric dimension. Instead, it spatially fixes the temporal movement of the hand stammering across the paper, blindly groping as if writing in the darkness, leaving an unreadable trace that oscillates between the attempt to remember the past and the incapability of mastering it. This 'trait' can decide neither between text and image, nor between past and present.

Pfeffer's handwritten letter is different, however. It would be readable if the museum's expository agent, the first person in the narrative of the museum's visit, did not impede our reading of the letter by covering it with a transparent label and then casting either too much or too little light on it. Whereas Pfeffer's letter is not allowed to unfold its alphanumeric dimension and thus speak for itself, Anne's diary is made readable without any obstructions. It appears as a tourist attraction displayed to re-present a historical past that is literally made tangible in an attic with glossy images, showcases, and videotapes. These 'visuals' serve to emphasize the museum's documentary reliability. Videotapes here are used as semiophores, that is, as documents or objects referring to an invisible, pre-existing meaning which they claim to re-present. For Pomian, the logic of the semiophore originates in its 


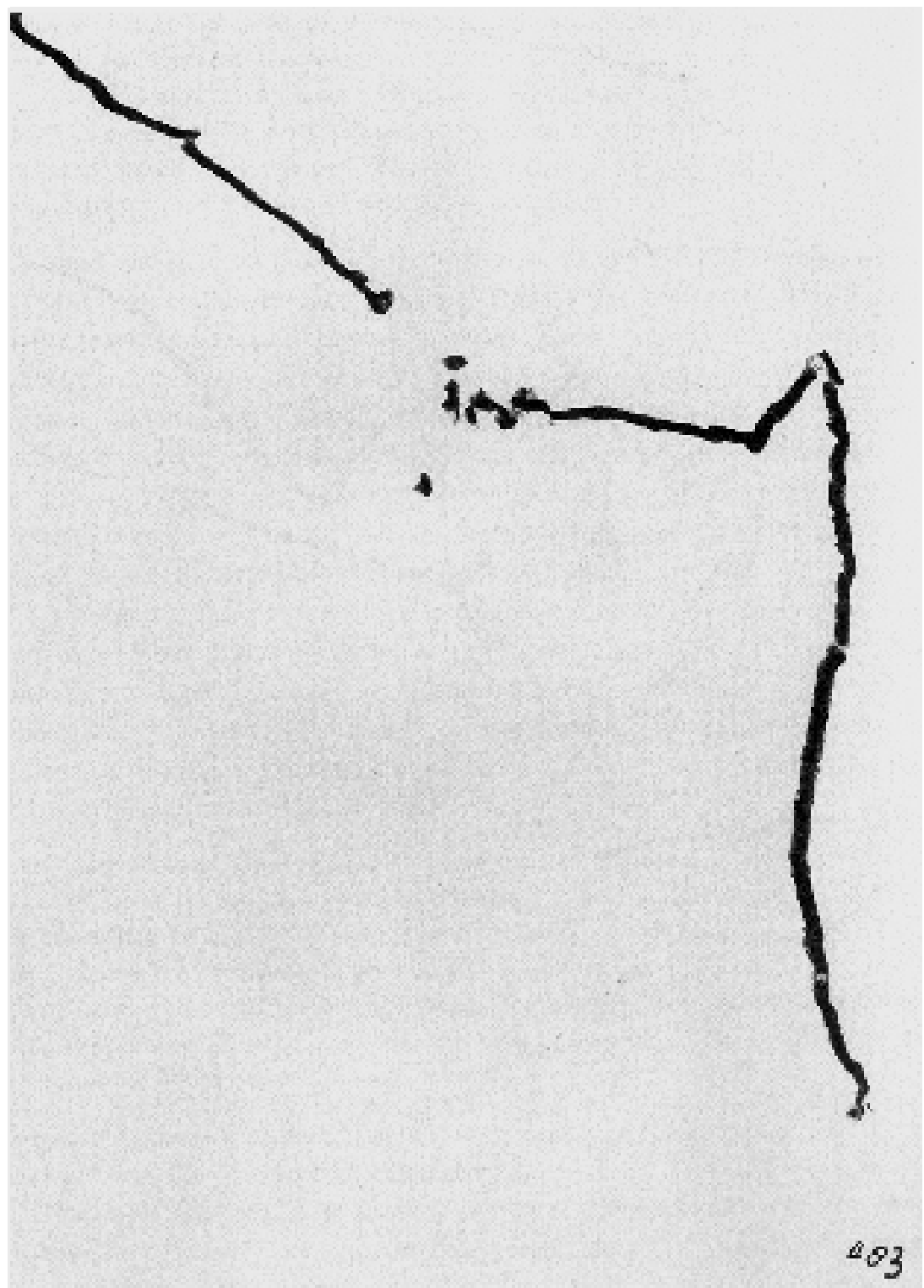

2. Armando, Drawing, 1983; pencil on paper, $18 \times 13 \mathrm{~cm}$

Collection Helen van der Mey, London 
religious function of connecting to the realm of the dead and the divine. ${ }^{23}$ This rhetoric of making the invisible visible turns the museum into a temple, an institution that makes the invisible truth visible, and an apparatus to produce ultimate authenticity. Whereas Pfeffer's letter is underexposed, overwritten by an authoritative act of drowning out his whispered declaration of love, Anne's diary seems overexposed. How much authenticity do we need to rescue us from amnesia? Can there be such a thing as too much authenticity?

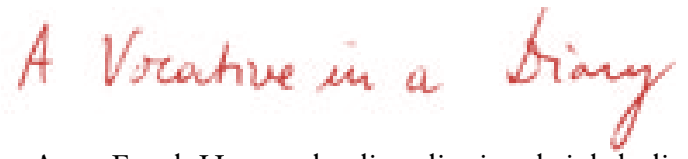

In the attic of the Anne Frank House, the diary lies in a brightly-lit showcase on a transparent lectern on top of a mirror. Thus exposed, the visitor is allowed to study the notebook from every angle, including its - otherwise hidden - hardcover, bound in orange-yellow checked textile, and to examine the notebook in its threedimensional compact and material shape. The diary lies open at a page that is composed like a layered paper collage, consisting of two leaves of paper taped to the diary's densely written pages, one of them containing a series of three portrait photographs of Anne Frank. The various units of text are written in slightly varying styles of childish, school-trained script, and is in some places overwritten with pencil corrections in a more mature, individual script, thus mirroring the process of writing over time. Each script is meticulous and beautiful. ${ }^{24}$

Between the text fragments that remain visible through the overlapping sheets of paper, I discover the phrase 'Beste Kitty' ('Dear Kitty') - a vocative in a diary. Here the practice of diary writing intermingles with that of private correspondence. But who is this Kitty? The museum does not mention her as one of the inhabitants in the secret annex. And yet she seems to be Anne's second person, the 'you' to whom she directs her discourse. The vocative often shows up in Anne's notes. As a pronoun, it is introduced in the very first lines of the diary: 'I hope I will be able to confide in you, as I have been unable to confide in anyone thus far, and I hope that you will be of great comfort to me. Anne Frank. 12th June 1942' (De dagboeken, 197; author's translation). ${ }^{25}$ Later on in the diary, this invented second person is named 'Kitty'. Kitty becomes an integral part of Anne's diary, her ideal reader and best friend. ${ }^{26}$

José van Dijck points out in her article on 'Diary Writing and Web Logging' elsewhere in this book that such dedication to an absent audience is more a rule than an exception in the diary genre, because 'writing, even as a form of self-expression, signals the need to connect, either to someone... else, or to oneself later in life'. Van Dijck concludes that ' [d]iary writing is, to a large extent, a cultural form firmly rooted in rhetorical conventions: intimacy and privacy are effects rather then intrinsic features of the genre'. Such public privacy also pertains to Anne Frank's diary. Anne repeatedly expresses her wish to be a journalist and a famous writer. An appeal from Dutch Minister Bolkestein on March 28, 1944 via the 'Oranje' radio channel in London to the people of Holland to keep diaries as historical documents for the postwar era inspires Anne to rewrite and edit her diary 


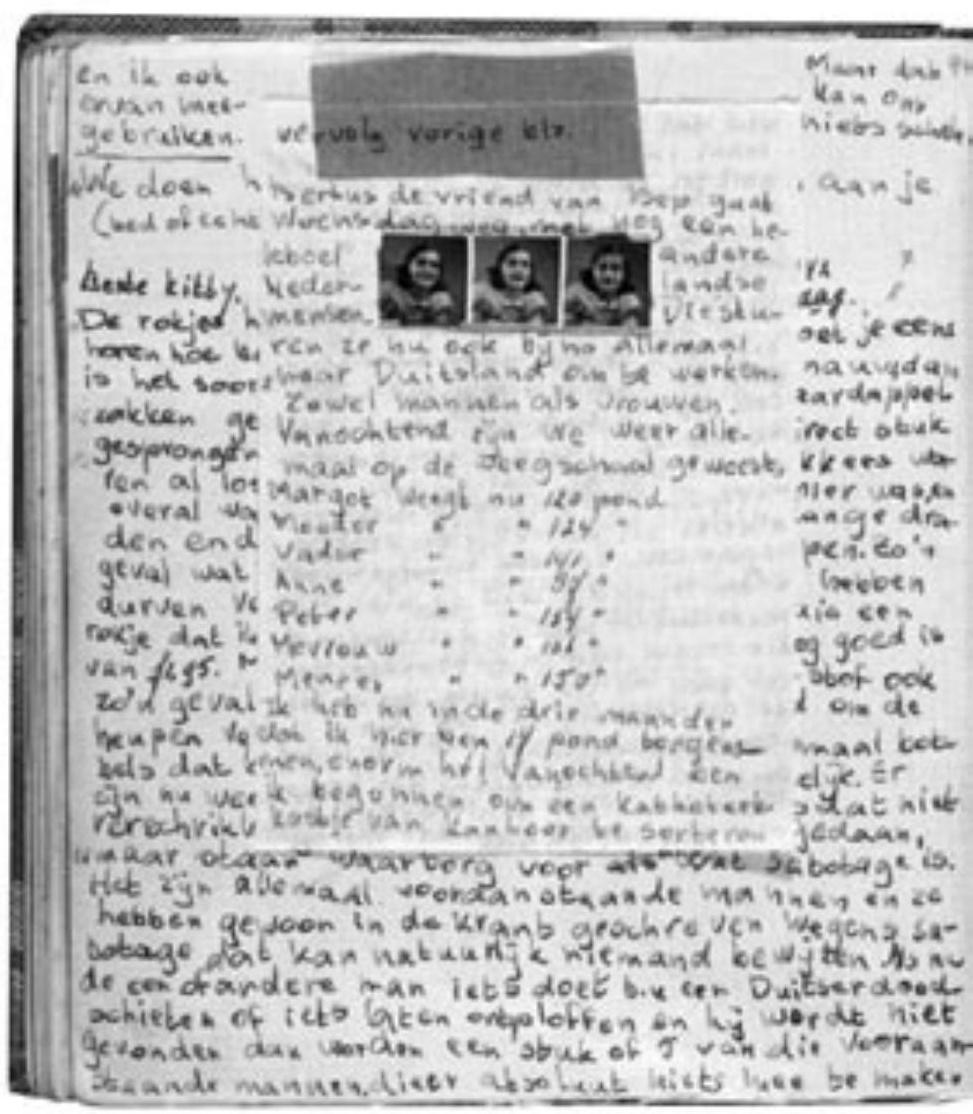

3. Anne Frank, diary page 20 October 1942

into a book called Het Achterhuis (Van der Stroom, 69-71). ${ }^{27}$ 'Dear Kitty', writes Anne one day after this appeal, 'Imagine how interesting it would be if I were to write a novel about the Secret Annex...' (De dagboeken, 594; author's translation). ${ }^{28}$ Her notes were meant to be published, at least partly.

The name Kitty is thus indeed readable as allographic text. ${ }^{29}$ As autographic manuscript in the museum's showcase, however, this vocative is highly ambivalent. On the one hand, the mystery of 'Kitty' impels the visitor to delve into the book's depths to learn more about her. But unlike the allographic print versions in the museum's shop, the autographic original manuscript in the showcase is not meant to be leafed through. Even though it is exposed on top of a mirror as a three- 


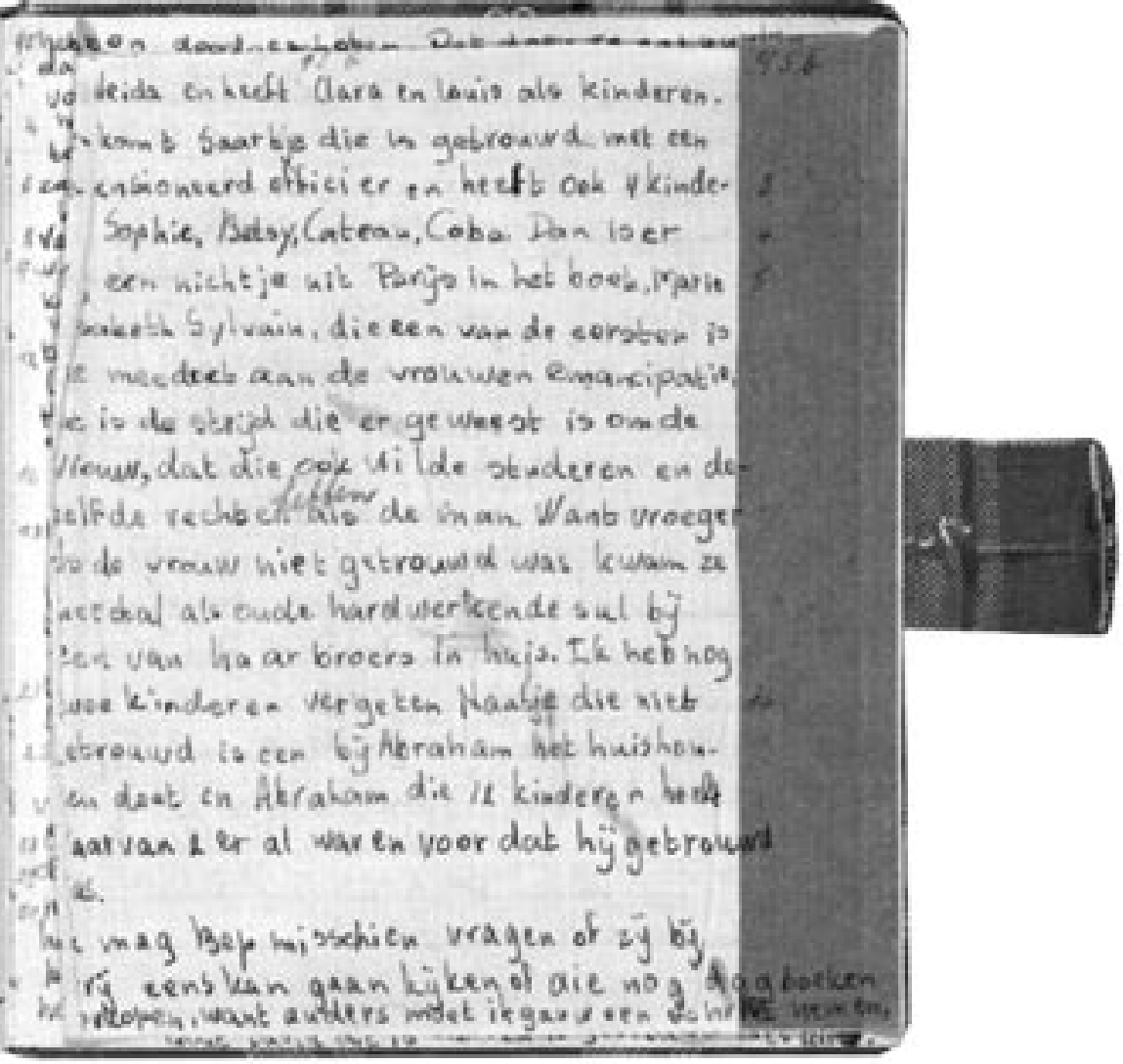

dimensional object, its readability is reduced to this flat double page on display. This volume actually has no volume. The proper name of Kitty cannot function unproblematically as an index pointing at a person. It has neither a referential depth nor a context in which it could become meaningful. The only reduced context it has is the one provided by the institutionalizing frame of the museum: the panopticon of the Holocaust. Thus exposed, the second person addressed by the diary's vocative remains flat and unreadable, deprived of other subject possibilities except as a witness offering evidence of Nazi crimes. Because 'Kitty' is not allowed to have alternative narratives, she is as much in danger of becoming a flat fetish, as I earlier argued regarding Anne. 
The logic of an imagined addressee put on display in a museum is so vastly complex because the addressee is simultaneously erased and produced by the visual rhetoric of the showcase. The more Kitty performs, the more she withdraws. Both as text and as image, 'Kitty' comes to represent something that is by definition absent. This empty signifier has no reference but is only deictic because it indicates nothing but the vacant place of a desired yet absent addressee who is constitutive for the here and now of the speech act. The moment Anne Frank's private diary becomes a public cultural object constituting shared history, we all - as readers and as visitors - participate in this second personhood. The reader or the visitor thus becomes Anne's intimate confidant - at least metonymically.

This metonymic second-person position is not without its problems, however. What is on display in the showcase is, after all, a private diary, and diaries, like love letters, are neither meant to be read aloud, nor to be displayed in public. They belong instead to the intimate private sphere, which deserves protection (see Roessler 2001). The desire of diary writers for a public audience thus appears somewhat exhibitionist. Analogously, the reader or spectator is in such an expository situation is encouraged to slip into the role of a voyeur who, herself invisible, gazes unhindered at an immediately available object. We look at Anne's private diary as an object whose voice is reduced to the notes of a single page. And we look at Anne's flat photo portrait showing her smiling at us from the museum wall with the subtle sadness of a saint to whom the visitor can direct her prayers: 'you who have died for us... forgive us the betrayer's debts...' Do we, as visitors, or voyeurs, expect her to be murdered? Do we thus murder her once more?30

The diary exposed on the mirror, is simultaneously voluminous and flat. Thus far, I have argued that this projection of its third dimension onto a two-dimensional mirror image silences the speech act of the object on display. When analyzing this expository gesture in detail, however, another aspect comes to the fore. Looking at the diary on top of the mirror it is striking to note not only this projection of depth, but the fact that, in the same glance, my gaze is caught by my own mirror image. This gaze hits me, like Barthes' punctum, as an unforeseen detail. It disturbs the museum's authoritative first-person speech act, which forces me to gaze unobstructed at a mute object. Here I am, Anne's second person, exposed in a showcase in her museum. My mono-directional gaze at Anne as the always already victim of Nazi murder is - like a dead letter - returned to the sender. Although I can choose to leave this showcase at any time, I realize that I have always already been included in it. Like Kitty, I come to stand in for a desired friend in a future speech act. For it is the basic principle of all writing, including handwriting, to function as a trace left to be read. It is only this relation of past and future, only this reciprocal gaze that allows for friendship. The speaker, then, can never be that author who is perceived as a pure and indivisible origin in its authentic state. It is only as authentic as the projection of my mirror image, exposing myself looking at Anne Frank, only as authentic as a projection of depth onto a flat surface. 


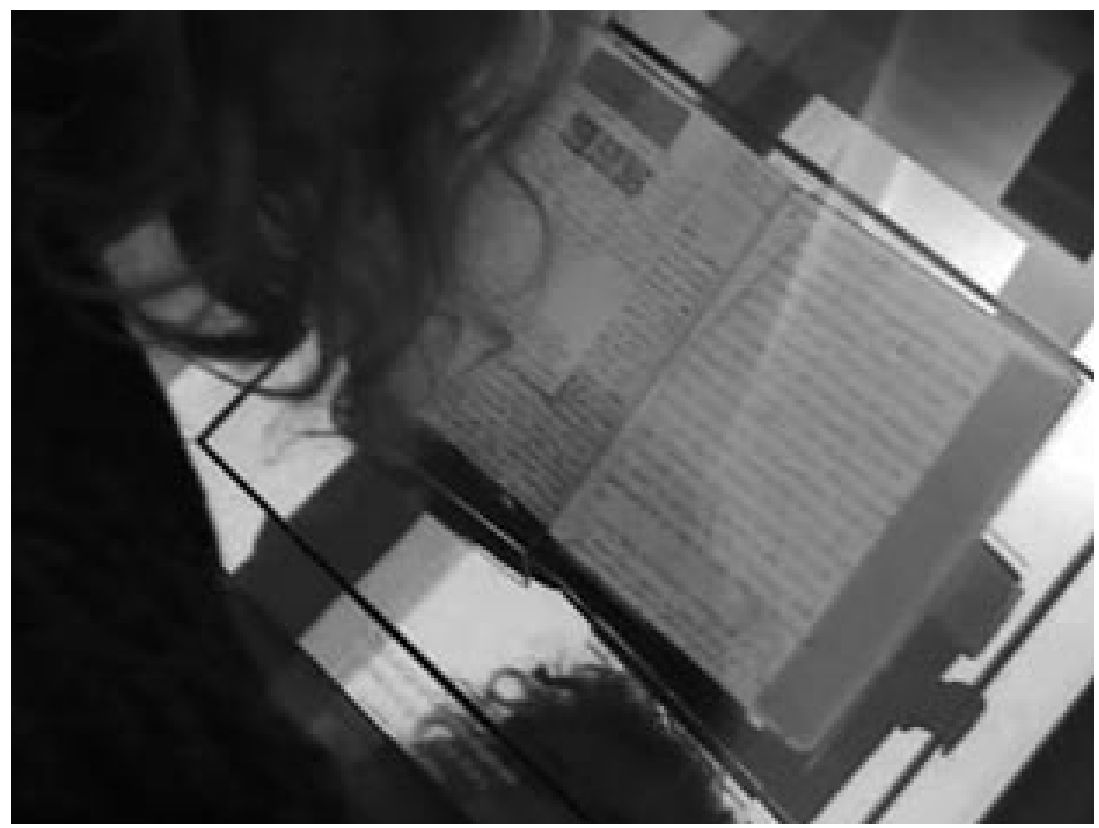

4. Anne Frank's diary
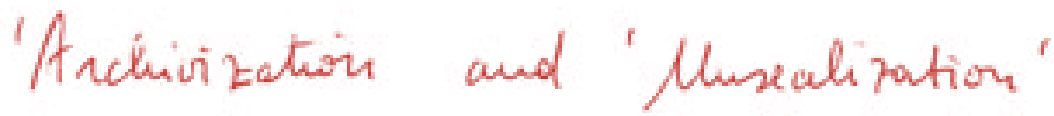

In her novel The Daughter, Jessica Durlacher has her heroine Sabine, an administrator at the Anne Frank House, comment on the museum:

It goes on being the secret annex here. It will never end. Ever. The house is eternally empty. This is the cruelty of it, the tragedy.... Actually, it is emptied out every day over and over again, this house. Every day, they are arrested once again. And every day, every morning when I come here, I find the house empty. And they are gone, once again. A horrible repetition, like in hell. ${ }^{3 I}$

In this passage, Sabine evokes an iteration of history as the return of a past event into her present experience. This miraculous recurrence is based on the logic of the memorial, which - unlike most museums and archives - is an authentic place where actual events really happened. In Sabine's words, this event qualifies, in a way, as a non-event as it is constituted by absence, by an intervention which does not happen. However, the 'cruel' and 'tragic' force of the abandonment of the secret annex as a non-event is at the same time grounded in the idea of presence, of a possible future that has not come true. The experience of loss of this other future, along 
with the knowledge about its possibility, turns into an act of mourning constituting a cultural memory of the Holocaust. The desire to participate in this public ceremony is the primary incentive for almost one million people a year to visit the authentic location of Anne Frank's house and to retrace her route, climbing the same stairs, peering into the darkness, and shuddering at the thought of betrayal.

And yet, I will argue that the recurrence of authenticity is permeated by a dialectics of counterfeit. For the visitor, the museum functions as a stage, and the diary in this play is a prop, in Latin a requisitus, which literally means a required or desired object. Unlike the innumerable print editions of The Secret Annex, the appearance of this musealized textbook has a sensational value. Its materiality physically strikes us. It turns the writing from a sober speech act into a tender whisper, as if the author speaks to us personally, murmuring her secrets into our ears exclusively. Handwriting, here, makes a difference, because it does not only inform us, but affects us. Paradoxically enough, this touch - although sensational and corporeal - is not indulged by material authenticity alone, because the diary in the case is, strictly speaking, not identical to the one manufactured by Anne Frank. As a material object, it is subject to time and to dilapidation; its constitution is different than it was 60 years ago. This is precisely why two perfect facsimile copies were manufactured. What needs to be preserved is, indeed, material likeness, to save the physical touch indulged by the original diary for when it is no longer there. It is only the auratic here and now of handwriting's public performance in the museum that allows the viewer to be affected by catharsis. Whoever wants to evoke the idea of authenticity can only apply for the authenticity of these affectionate and physical effects - effects that are by definition theatrical. On the one hand, these effects result from a putatively inescapable directness and presence that distinguishes the medial space of the stage or the museum from that of film or photography. On the other hand, this stage, as a theatrical setting, can never be the authentic place for a museum visitor as it once was for Anne, because in its recurrence it can be left whenever one feels like it.

The magical force of the memorial is not grounded in a representational practice, for the past can never be made present, neither by authentic documents, nor by authentic places. Rather, the memorial functions as a performative. ${ }^{32}$ In his study on Holocaust Effects in Contemporary Art, Literature, and Theory, Ernst Van Alphen argues that:

the Holocaust is not re-presented, but rather presented or enacted. In terms of speech-act theory I might explain it differently. The Holocaust is not made present by means of a constative speech-act - that is, as a mediated account, as the truthful or untruthful content of the speech act; rather, it is made present as performative effect. Those performative acts 'do' the Holocaust, or rather, they 'do' a specific aspect of if. (10)

As much as we desire authenticity and however urgent it may be in our culture, historical authenticity is always a product of - and I need to use two neologisms 
here - either 'archivization' or 'musealization'. The term 'archivization' is used by Derrida in Archive Fever to indicate that the archive 'produces as much as it records the event' (17). In addition, historical authenticity can also be the result of what Eric Ketelaar calls 'archivalisation'. Ketelaar $(1998,10)$ points out that the English term 'archivization' is a translation of the French neologism 'archivation' used by Derrida in the original version. Following Derrida, Ketelaar $(1998,10)$ distinguishes the term 'archivalisering' (in Dutch) from 'archivization', adding another syllable enclosing the concept of 'value' into the concept of the archive, to emphasize that choices made in archiving are highly dependent upon cultural values. 'Archivalization' is to be seen 'not only in the technological sense, as Derrida understands it, but especially in the socio-cultural sense, as in the examples by Trouillot and Stoler. In one culture they decide that the birth of a baby is an archivable fact and record it, in another [i.e., in slavery] they don't' $(1999,55)$. In his article 'Writing Archival Machines' (elsewhere in this volume) Ketelaar specifies that 'archivalization' means 'the conscious or unconscious choice (determined by social and cultural factors) to consider something worth archiving' (emphasis Ketelaar). The archival adventure, thus considered, commenced at the very moment that Anne Frank decided to keep a diary, while in the Hitler case, it was the failure to make such a decision, which, in retrospect, set the stage for Kujau's forgeries. ${ }^{33}$

The museum, in a way, functions in the same way. I will use the term 'musealization' as analogous to 'archivization' to indicate that the 'musealized' object is not an object, but an event. 'Musealizing' means 'to do' the object. My analysis of handwritten objects in this paper gives rise to a conception of handwriting that does not 'mute' objects in the museum exposed by an authoritative first person, the curator, to a second person, a (voyeuristic) visitor - to echo Bal's theory of exposition. Archival work - despite its efforts to define the difference between true and false - is as performative as the act of musealization. Both institutions aim at dedicating the past to the future; they are - as Derrida has called it - 'an injunction to remember' $(1995,33)$. Thus seen, they both imply two temporalities sliding into another: a past present that is confirmed by the archive, salvaged like in Noah's Ark, and at the same time relaunched en abyme, for a document in the archive no longer fulfils its original function. Cultural or historical memory is an act taking place in the theatrical abyss between past and present.

In the case of the reception of the alleged Adolf Hitler diaries, the anachronistic structure of memory was totally denied, disregarding the fact that these diaries were false. Handwriting served as a fetish to directly and unreflectingly conflate past and present. The situation is more complex in the case of Anne Frank's diary. Not only because her diaries were acknowledged as true, but - I hope to have shown here - because it is not enough to ground our recall of the past in a positivistic understanding of authentic origin. Such an understanding risks harming the past, like certain chemical methods deployed to prove a document's authenticity threaten to destroy this very document. It is not sufficient to shape the act of memory as an act of certification and classification by exposing documents as either true or false, for this reduces the work of the archive and the museum to an act of 
re-'collecting' the past, the fetishist quality of which I have discussed in this paper. Documents can make history present, but never quite so - neither horizontally as documentary text, nor vertically as imaginative image. The only effect they can achieve is re-iteration, as it is inscribed in all writing, including handwriting - that is, iteration with a difference.

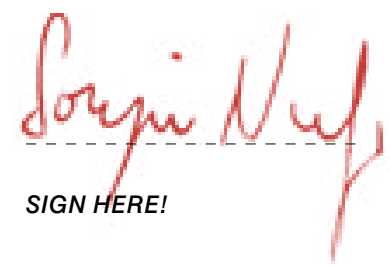


Notes

1. This essay has benefited greatly from Eric Ketelaar's stimulating and inspiring comments and criticism. I would also like to acknowledge José van Dijck and Esther Peeren for their many suggestions, as well as the support received from the Anne Frank House.

2. The immense authority of the archive as a host of authenticity can be illustrated by the term 'official custody', which is defined by Sir Hilary Jenkinson in his Manual of Archive Administration $(1922,10)$ as the basic condition for public archives. Custody implies the acceptance of a certified copy of a document from the body of the Public Record Office in London in any court of law. For a document in the British Museum to receive this credence, however, the judge requires the presentation of the original document.

3. These involve the Stielau case in Lübeck (1960), the Roth case in Frankfurt (1977), the Schönborn case in Frankfurt and the Kunth case in Stuttgart (both 1979), and finally the Römer case in Hamburg (1993). A detailed overview of the protracted debate on the authenticity of this diary is provided by David Barnouw 'Aanvallen op de Echtheid van het Dagboek' in De dagboeken van Anne Frank. 99-119, 1990.

4. The material concerning the scandal of the Hitler forgeries is immense. Numerous articles were published in newspapers worldwide as well as on the Internet (see $<$ http://fortunecity.com/dikigoros/schtonk. htm $>$ and <www.sniggle.net/kujau.php >). The film (1992, Dir.: Helmut Dietl) unravels the story of the canard in detail, and the case has become the subject of information and archival studies (for an overview, see e.g., Picker and In 't Veld).

5. <www.sniggle.net/kujau.php >.

6. For Hitler's diaries, the process of exposing their falseness can be divided into two phases. Josef Henke, archivist of the Bundesarchiv (German National Archive), defines the first phase as running from April 5, 1982 - when Thomas Walde and Leo Pesch from Stern first contacted the Bundesarchiv - until April 22, 1983 when Stern announced the discovery of the lost Hitler diaries. The second phase runs from April 25, 1983 - when three diaries were given to the Bundesarchiv for certification of their authenticity - until May 6, 1983, when the German Minister of the Interior declared the diaries to be forgeries (Henke, 289).
7. Hugh Trevor-Roper, quoted from

$<$ www sniggle.net/kujau.php $>$.

8. More precisely, they identified perlon, also known as polyamide 6 , in the bindings of the volumes dating from 1934, 1941, and 1943 , as well as polyester fibers in the 1941 volume. Perlon was not produced until after 1943, and polyester not before 1953. Moreover, by means of ultra-violet irradiation, they discovered paper-bleaching agents in the paper from the 1941 and 1943 volumes that were not used prior to 1945 (Czichos, 78-79; Franke). Another flaw was that the diaries had a monogram on the front cover that turned out to be plastic. Moreover, it was composed of the letters ' $\mathrm{FH}$ ' instead of 'AH'.

9. Several assessments by acknowledged handwriting experts were carried out on the handwriting of Anne Frank, among which one by the Bundeskriminalamt (the German Criminal Court Laboratory), and, more extensively and authoritatively, by the Gerechtelijk Laboratorium (Dutch Forensic Laboratory) in Rijswijk, commissioned by the Netherlands Institute for War Documentation, or NIOD (Nederlands Instituut voor Oorlogsdocumentatie, the Dutch Institute for War Documentation). 10. In 1986, the complete diaries of Anne Frank and the positive results of the laboratory research were published under the title De dagboeken van Anne Frank (The Diaries of Anne Frank).

11. 'Met aan zekerheid grenzende waarschijnlijkheid afkomstig van de producente van het vergelijkingsschrift, Anne Frank' (164).

12. This idea comes close to Michel Foucault's conception of the archive as epistemological system, as 'the general system of the formation and transformation of statements' (146). The archive 'is that which gives to what we can say - and to itself, the object of our discourse - its mode of appearance, its forms of existence and coexistence, its system of accumulation, historicity, and disappearance' (145-146).

Wolfgang Ernst summarizes this as follows: 'Foucault defines the archive as referring to neither the sum of all transmitted documents, nor to the institution of this transmission. Rather, it refers to the system dominating the appearance and the actual functioning of the utterances.' ['Foucault definiert als Archiv weder die Summe aller überlieferten Dokumente 
noch die Institution ihrer Überlieferung, sondern das System, welches das Auftauchen sowie das weitere aktuelle Funktionieren der Aussagen regiert.']

(16; author's translation).

13. 'Dieses... Material hatte nur einen kleinen Schönheitsfehler: Große Teile stammten aus derselben Werkstatt, in der auch die Hitler-Tagebücher angefertigt worden waren. Die Experten... stellten [also] fest, tatsächlich, die stammen aus derselben Feder' (Bissinger, 173).

14. See also José van Dijck, 'Of Diaries and Weblogs', elsewhere in this book. 15. The reproduction of photographs can be seen as symptomatic of the process of the facsimilation of the diaries in general. In his famous essay, Benjamin regrets the loss of aura and relates it to 'the first truly revolutionary means of reproduction, photography.... To an ever greater degree, the work of art reproduced becomes the work of art designed for reproducibility. From a photographic negative, for example, one can make any number of prints; to ask for the "authentic" print makes no sense' (224). As opposed to this, Benjamin in his 'Small History of Photography' ('Kleine Geschichte der Photographie'), proposes that early photography would be the auratic art par excellence, particularly Atget, who would not fake a false aura in his photographs. Aura, thus considered, does not depend on a unique, irreducible print. Rather, it can unfold in repetition, as long as it remains 'a peculiar gossamer of space and time: a unique phenomenon of distance, as close as possible' ('ein sonderbares Gespinst von Raum und Zeit: einmalige Erscheinung einer Ferne, so nah sie sein mag') (57; author's translation). 16. For the wide cultural implications of this statement see Hillel Schwartz's monumental The Culture of the Copy.

17. Marx's terms commodity and fetish form the foundation of a series of critical studies. Bal (1994, 107-110) develops her concept of collecting by critically discussing the seminal studies of fetishism - for a Freudian approach - by Mitchell (160-208), and - for a Lacanian approach - by Žižek (32-45). For the idea of fetishism as an archival attitude, see Helen Wood, 'The Fetish of the Document: An Exploration of Attitudes Towards Archives'.

18. Pomian has made the concept of semiophores travel from the museum to the archive. For a reinterpretation of Pomian's dialectics of the visible and the invisible in both the museum and the archive, which goes beyond the idea of re-presentability of history, see Ketelaar (2003).

19. Marianne Hirsch describes the memory of the Holocaust by the second generation as 'postmemory': 'Postmemory characterizes the experience of those who grow up dominated by narratives that preceded their birth, whose own belated stories are displaced by the stories of the previous generation, shaped by traumatic events that they can neither understand nor recreate' (8).

20. Or - to echo Freud - 'belated'.

21. The literature on the problem of traumatic recall is immense. Van Alphen (1999) explains the difficulties of giving rise to subjectivity because this would imply confessing one's own guilt. See also Mieke Bal's 'Introduction' in Acts of Memory (1999).

22. Mitchell's focus is not on Holocaust experiences, but on slavery. When applying his conceptualization of 'imagetext' to Holocaust experiences, I do not intend to compare these two historical fields because this would imply a denial of the very specific quality of each of them.

23. Particularly in his chapter 'Collections: le visible et l'invisible' in Pomian, 1987, 15-58.

24. I have discussed the 'drama' of handwriting in detail in my book Kalligramme. Zur Medialität einer Schrift (Calligrams: The Mediality of Writing) by arguing that the kalos of handwriting would turn writing from an almost invisible medium into a vivid, corporeal actor of cultural agency. 25. 'Ik zal hoop ik aan jou alles kunnen toevertrouwen, zoals ik nog aan niemand gekund heb, en ik hoop dat je een grote steun voor me zult zijn. Anne Frank. 12 juni 1942'.

26. For the dialogical relationship between Anne and Kitty see Brouwers, 7-8.

27. This doubling of versions and scripts added fuel to the fire of the assailants of the diary's authenticity. For this reason, the Dutch Rijksinstituut voor oorlogsdocumentatie commissioned the elaboration of a historical-critical text edition containing Anne's first version (A), her second version (B) as well as the final version that Otto Frank edited for publication (De dagboeken van Anne Frank. 1990, Harry Paape, Gerrold van der Stroom and David Barnouw (eds.), Rijksinstituut voor Oorlogsdocumentatie, 189-736).

28. 'Stel je eens voor hoe interessant het 
zou zijn, als ik een roman van het Achterhuis uit zou geven...'.

29. When pasting the text dimension into this image, the name Kitty invites us - for a change - to not only focus on what the diary does not tell us but on what it does tell us. Brouwers (5-7) emphasizes that the main topic of the diary is the processual formation of identity along with a young girl's awakening sexuality and the awakening self-consciousness of a beginning writer. The diary also contains a multiplicity of literary, picturesque, and ironic short stories. Both this intimate and this comical dimension of the notebooks redirect our view of Anne and protect her from being flattened into a two-dimensional image. 30. For the politics of the gaze as an agent of power see Norman Bryson, chapter 5, 'Gaze and Glance' in Vision and Painting. As for the situation of the logic of the museum's gaze, see Bal, Double Exposures, especially chapter 8, 'His Master's Eye'. 31. 'Het gaat hier maar door met achterhuis te zijn. Dat gaat nooit meer weg. Nooit meer. Het is voor altijd leeg hier. Dat is zo wreed, zo tragisch.... Eigenlijk wordt het elke dag opnieuw leeggehaald, dit huis. ledere dag worden ze opnieuw opgepakt.
En elke dag, elke morgen als ik hier kom, tref $i k$ het huis leeg aan. Zijn ze weg, alweer. Een gruwelijke herhaling, net als in de hel' (13; author's translation).

32. The concept of performativity originates from 19th-century ethnology where it describes rites and festivities. In the 20th century, it became prominent in theatre studies as a concept for describing onetime events that could not be covered by the traditional concept of 'text'. In philosophy, Austin (1975) applies the concept to linguistic utterances as acts that 'do' rather than state things. The event-character of the concept was profoundly revisited by Derrida in Signature Event Context by insisting on the citationality or repeatability of the signature as a singularity to be iterated. The idea that repetition involves both similarity and difference, and that it therefore allows for other possibilities giving rise to individual subject interventions, or agency, became the founding principle of gender studies (Butler 1993), and, again, for philosophy (Rössler 2001).

33. Moreover, the archive 'archivalizates', for example, by exposing events and records as either true or false.

Works Cited

Alphen, Ernst van. Caught by History: Holocaust Effects in Contemporary Art, Literature, and Theory. Stanford: Stanford University Press, 1997.

- 'Symptoms of Discursivity: Experience, Memory, and Trauma', in: Bal, Mieke, Jonathan Crewe, and Leo Spitzer (eds.), Acts of Memory. 24-38, 1999.

Austin, J.L. How to do Things with Words. Cambridge: Harvard University Press, 1975.

Bal, Mieke. 'Telling Objects: A Narrative Perspective on Collecting', in: John Elsner and Roger Cardinal (eds.), The Cultures of Collecting. London: Reaction Books, 1994.

- Double Exposures: The Subject of Cultural Analysis. New York and London: Routledge, 1996.

- 'Introduction', in: Bal, Mieke, Jonathan Crewe, and Leo Spitzer (eds.), Acts of Memory, vii-xvii, 1999.

Bal, Mieke, Jonathan Crewe, and Leo Spitzer (eds.). Acts of Memory: Cultural Recall in the Present. Hanover, NH and London: University Press of New England, 1999.

Butler, Judith. Bodies that Matter: On the Discursive Matters of 'Sex'. New York: Routledge, 1993.

Barnouw, David. 'Aanvallen op de echtheid van het dagboek', in: Frank, Anne, De dagboeken van Anne Frank, 99-119, 1990.

Benjamin, Walter. 'The Work of Art in the Age of Mechanical Reproduction', in: Hannah Arendt (ed.), Illuminations. 217-251. Trans. Harry Zohn. New York: Schocken, 1969 (1936).

- 'Kleine Geschichte der Photographie', in: Das Kunstwerk im Zeitalter seiner technischen Reproduzierbarkeit. 45-64. Frankfurt am Main: Suhrkamp, 1977 (1931).

Bissinger, Manfred. Hitlers Sternstunde. Kujau, Heidemann und die Millionen. Hamburg: Rasch und Röhring, 1984.

Brouwers, Ton. 'Anne Frank', in: Zuiderent, Ad, Hugo Brems and Tom van Deel (eds.), Kritisch Literatuur Lexikon. Amsterdam: Martinus Nijhoff, 2002. 
Bryson, Norman. Vision and Painting: The Logic of the Gaze. London: Macmillan, 1983.

Czichos, Horst. Was ist falsch am falschen Rembrandt? Und Wie hart ist Damaszener

Stabl? Wie man mit Technik Kunst erforscht, prüft und erhält. Berlin: Nicolai, 2002.

Derrida, Jacques. 'Signature Event Context', in: Limited Inc. 1-21. Evanston, IL:

Northwestern University Press, 1988.

- Archive Fever: A Freudian Impression. Trans. Eric Prenowitz. Chicago and London: University of Chicago Press, 1995.

Dijck, José van. 'Writing the Self: Of Diaries and Weblogs', in: Neef, Sonja, José van Dijck, and Eric Ketelaar (eds). Sign Here! Handwriting in the Age of New Media. Amsterdam: Amsterdam University Press, 2006.

Domarus, Max. Hitlers Reden und Proklamationen, 2 vols., [s.l.], 1962-63.

Durlacher, Jessica. De dochter. Roman. Amsterdam: Bezige Bij, 2000.

Ernst, Wolfgang. Das Rumoren der Archive. Ordnung aus Unordnung. Berlin: Merve, 2002.

Foucault, Michel. The Archaeology of Knowledge. Trans. A.M. Sheridan Smith. London and New York: Routledge, 2002 (1972).

Frank, Anne, De dagboeken van Anne Frank. ed. Paape, Harry, Gerrold van der Stroom, and David Barnouw. Rijksinstituut voor Oorlogsdocumentatie. Amsterdam: Bakker, 1990

Franke, Werner. 'Papierbezogene Echtheitsprüfung der sogenannten "Hitler-Tagebücher"', in: BAM-Jahresbericht 1983, 1983.

Freud, Sigmund. 'Fetischismus', in: Studienausgabe. Volume 3. Psychologie des Unbewussten, 379-389. Frankfurt am Main: Fischer, 2000 (1927).

Goodman, Nelson. Languages of Art: An Approach to a Theory of Symbols. Indianapolis: Hackett Publishing Co., 1976.

Hamilton, Charles. The Hitler Diaries: Fakes that Fooled the World. Lexington: University of Kentucky Press, 1991.

Hardy, J.J. 'Samenvatting van de resultaten van het handschriftvergelijkend en documenttechnisch onderzoek van wat bekend staat als het dagboek van Anne Frank', in: De dagboeken van Anne Frank, 121-186, 1990.

Henke, Josef. 'Die sogenannten Hitler-Tagebücher und der Nachweis ihrer Fälschung. Eine archivfachliche Nachbetrachtung', in: Friedrich P. Kahlenberg (ed.), Aus der Arbeit der Archive. Beiträge zum Archivwesen, zur Quellenkunde und zur Geschichte, 287-317. Boppard am Rhein: Boldt, 1989.

Hirsch, Marianne. 'Projected Memory: Holocaust Photographs in Personal and Public Fantasy', in: Bal, Mieke, Jonathan Crewe, and Leo Spitzer (eds.), Acts of Memory, 3-23, 1999.

In 't Veld, N. 'De dagboeken van Hitler', in: Dittrich, Naarden, and Renner (eds.) Knoeien met het verleden. Utrecht and Antwerp: Spectrum, 1984, 176-189.

Jenkinson, Hilary. A Manual of Archive Administration. Oxford: Clarendon, 1922.

Ketelaar, Eric. 'The Power of the Past. Visibilities and Invisibilities in Archives, Libraries and Museums'. Keynote address at Kildenes Makt, a conference organized by AвмUtvikling, the Norwegian Archive, Library and Museum Authority, held in Oslo, September 2003.

- Archivalisering en Archivering. (Inaugural address at the accession to the Chair of Archivistics at the University of Amsterdam). Alphen aan den Rhijn: Samson, 1998.

- 'Archivalisation and Archiving', in Archives and Manuscripts. (27), 54-61, 1999.

- 'Writing Archival Machines', in Neef, Sonja, José van Dijck, and Eric Ketelaar (eds). Sign Here! Handwriting in the Age of New Media. Amsterdam: Amsterdam University Press, 2006.

Marx, Karl. Capital. Volume I. Trans. Ben Fowkes, Intro. Ernest Mandel. Harmondsworth: Penguin, 1976 (1867).

Mitchell, W.J.T. Picture Theory: Essays on Verbal and Visual Representation. Chicago and London: University of Chicago Press, 1994.

Neef, Sonja. Kalligramme. Zur Medialität einer Schrift. Anhand van Paul van Ostaijen's De feesten van angst en pijn. Amsterdam: AsCa Press, 2000.

O’Toole, James M. 'On the Idea of Uniqueness', in: American Archivist 57. (Fall 1994), 632-688. 
Picker, Günther. Der Fall Kujau. Chronik eines Fälschungsskandals. Frankfurt am Main: Ullstein, 1992.

Pomian, Krysztof. 'Pour une histoire des sémiophores. A propos des vases des Medicis', in: Le Genre Humain, 14. Paris: Éditions de l'École des Hautes Études en Sciènces Sociales, 1986.

- Collectionneurs, amateurs et curieux. Paris: Éditions de l’École des Hautes Études en Sciences Sociales, 1987.

- Collectors and curiosities: Paris and Venice, 1500-1800. Cambridge: Polity, 1990

Price, Billy. Adolf Hitler: The Unknown Artist. Houston: Billy F. Price, 1983.

Rössler, Beate. Der Wert des Privaten. Frankfurt am Main: Suhrkamp, 2001.

Rühle, Gerd. Das dritte Reich. Dokumentarische Darstellung des Aufbaus der Nation. Berlin: Hummel.

Schwartz, Hillel. The Culture of the Copy: Striking Likenesses, Unreasonable Facsimiles. New York: Zone Books, 1998.

Silva, Teresien da. 'Denial of the Authenticity of the Diary', available at: <http://www: annefrank.org/content.asp?PID=426\&LID=2>, Februari 2006.

Stern. 1983, 18 (28 April); 19 (5 May); 20 (11 May); 21 (19 May); and 22 (26 May). Hamburg: Gruner \& Jahr.

Stroom, Gerrold van der. 'De dagboeken, "Het Achterhuis" en de vertalingen', in: Frank Anne, De dagboeken van Anne Frank. 69-97, 1990.

Tanja, Jaap. 'Anne Frank's Diaries in Facsimile', availeble at: <http://www:annefrank.org/ upload/downloads/auniqueset.doc>, Februari 2006.

Wood, Helen. 'The Fetish of the Document: An Exploration of Attitudes towards Archives', in: Margaret Procter and C.P. Lewis (eds.), New Directions in Archival Research. 20-48. Liverpool: Liverpool University Centre for Archive Studies, 2000.

Žižek, Slavoj. The Sublime Object of Ideology. London: Verso, 1989. 


\section{The Authority of Drawing: Haw Authenticity, and Authorship}

The idea of an 'authentic copy' that forms the focus of this book's chapter, is based on a contradiction; on what is rhetorically termed a contradictio in adiecto: 'authenticity' is generally associated with genuineness, originality, uniqueness. In other words, with something that cannot or should not be reproduced or that could only be 'copied' by losing its character, its specificity; in short, its sense. Thus, we can refer to cultural theories, for example on the decline of media societies - as represented, in a first and superficial impression, by someone like Walter Benjamin that are based on the opposition of, on the one hand, a pre-medial world of authenticity and the aura of the original and, on the other, the realm of media-technical reproduction of what may be called a copy, surrogate, simulacrum, dummy or, nowadays, simply fake.

Conversely, we know that if we try to provide a definition of the originality of the authentic, we enter into a cycle of reaffirmations of the 'one', only now in a chain of substitutions or supplements. I remember a paradoxical formulation I found in an art advertisement from the 1970s, when very expensive reproductions of masterpieces, produced by means of a new printing technique, were offered with the slogan: The absolute authentic reproduction.

We may laugh at this absurdity, but even a short look at the etymological context of the word 'authentic' teaches us more about both the original relationship of the concept and the question of handwriting or, as we say, authentication by palm/ finger: the definition of authentes refers to the concept of a 'master', a 'potentate', someone who makes something with his own hands as originator: an author. This is why it is often used as an adjective for the noun 'auctoritas' (authority). But all this is only representable by repetition, revealed as something identical within difference. Its authority derives from iteration, domination, and force: as a disposi- 
tion by a strategy of application and appropriation. As, for instance, in Kant's definition of authentic theodicy as exegesis, given by the legislator himself, an argument that always comes too late and that refers to a founding, establishing moment in the sense of donating as giving an origin which eternally lacks. And even though this origin is absent, it plays a fundamental role in the presence (Kant 1964, 116).

To cut up the complex and over-determined aporia and at the same time to precipitate forward: we are at once confronted with the abyss of a less mediatic and more metaphysical or, even better, theological question of creation: the monopolizing, monotheistic god who has to split, to differ, to double, or to reproduce himself in what we call the creation as revelation for the sole purpose of knowing about himself as the one and only god. But let us stop our speculation here, before we get sick of Hegelian dialectics.

The passage from the etymological context to the historical development of authenticity is perfectly analyzed in Lionel Trilling's book Sincerity and Authenticity. Trilling argues that in the beginning of the 16th century, the concept of sincerity was the first to claim a kind of moral or political correctness against a world of betrayal and falsification. A look at the word's etymological roots may be informative here:

The word... derived from the Latin word sincerus and first meant what the Latin word means in its literal use - clean, or sound, or pure. An old and merely fanciful etymology, sine cera, without wax, had in mind an object of virtu which was not patched up and passed off as sound, and serves to remind us that the word in its early use referred primarily not to persons but things, both material and immaterial.... But it soon came to mean the absence of dissimulation or feigning or pretence. (Trilling 1972, 12)

On the contrary, the word authenticity enlarges the range of legitimacy of pure origin:

I can rely on its suggesting a more strenuous moral experience than sincerity does, a more exigent conception of the self and what being true to it consists in, a wider reference to the universe and man's place in it, and a less acceptant and genial view of the social circumstances of life.... Conversely much that culture traditionally condemned and sought to exclude is accorded a considerable moral authority by reason of the authenticity claimed for it, for example disorder, violence, unreason. (Trilling 1972, 11)

In this sense, authenticity is attributed more to strategies of transgression and excess, but also, in an aesthetic sense, of artistic creation by destruction. Trilling recalls again the Greek etymology:

Authenteo: to have full power; also, to commit a murder. Authentes: not only a master and a doer, but also a perpetrator, a murderer, even a self-murderer, a suicide. These ancient and forgotten denotations bear upon the nature and intention of the artistic culture of the period we call modern. (Trilling 1972, 131) 


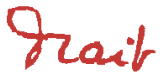

I am concerned with these aspects because I would like to concentrate more on the aesthetic point of view, especially in the form of a comparison between the artistic strategy of producing authenticity within so-called modernity and the juridical analysis of the underlying concept of authority.

And, of course, this is an article about the hand, about what is at hand or to hand.

But first of all I would like to invoke the basic theory of difference as delay, as trace, as developed by Jacques Derrida in his first great book, Of Grammatology. The main argument is - and here I have to be unpardonably short - that sense is not given as the representation of a former and original truth, but that it is produced by a real and material movement in the context of writing as tracing. We quickly see how Derrida subscribes to a long anti-Platonic tradition, to the critique of the metaphysical model of archi-idea and ektypos of sensorial appearance (noumenon and phaenomenon). We know the important stages of a kind of pre-histoire, such as Kant's model of schematism, which is also built on the idea of tracing a line to connect concepts to their apperceptions (a move later recycled in the linguistic turn of Humboldt's theory of human language). But what remains a crucial thesis, which Derrida never stopped repeating for more than 30 years, is the paradigm of iteration as supplement: the so-called archi-trace that is something that reveals itself only in the infinite difference ('differance') from something else, as an effect of dehiscence or, in different but no less confusing words, the trace is testimony only to the absence of which it is an index.

Derrida's writing on art, especially his work on painting and 'architexture', also claimed the difference between the picture as drawing and the act of drawing as the production or path-breaking (as in pushing forth) of visibility as trait. This difference, I will argue, applies for handwriting as much as for drawing, as both practices rely on the activity of a creative hand. The drawn visible mark refers to an invisible potency, a potency (as irreducibly unseen, invisible) of the trait, neither as the opposite of vision, nor as reproduction of an original vision, but rather as the invention of visibility (invention of the other; Derrida 1987). Thus, as Derrida argued in 'Memoirs of the Blind', painting, like writing, is the result of an unconscious, blind process of drawing:

Whether it be improvised or not, the invention of the trait does not follow, it does not conform to what is presently visible, to what would be set in front of me as a theme. Even if drawing is, as they say, mimetic, that is, reproductive, figurative, representative, even if the model is presently facing the artist, the trait must proceed in the night. It escapes the field of vision. Not only because it is not yet visible, but because it does not belong to the realm of the spectacle, of spectacular objectivity - and so that which it makes happen or come about cannot itself be mimetic. The heterogeneity between the thing drawn and the drawing trait remains abyssal, whether it be between a thing represented and its representa- 
tion or between the model and the image. The night of this abyss can be interpreted in two ways, either as the eve or the memory of the day, that is, as a reserve of visibility (the draftsman does not presently see but he has seen and will see again: the aperspective as the anticipating perspective or the anamnesic retrospective), or else as radically and definitively foreign to the phenomenality of the day. This heterogeneity of the invisible to the visible can haunt the visible as its very possibility. (Derrida 1993, 45)

The important step of representation, therefore, will be to re-appropriate the revealed form or figure as possibility, an act that constitutes the so-called authorship as domination of the work, which is always belated due to the withdrawal and inaccessibility of the trait. The signature as appropriation does not belong to the signing subject: what may be called 'deconstruction' in visual arts as a condition of construction implies this experience of decentralization, of the dislocation of the presence in a work of traces. The signature does not belong to the work, it is something different, foreign, a name which is part of a discourse on the work and which constitutes the relationship between the mere existence of a piece of work and the artist as inventor or producer not by the mere act of signing, but by referring to the whole sociological context of reception, publication, attribution, acceptance, recognition, etc. There is no signature without countersignature, as Derrida explained in an interview:

The origin of the work ultimately resides with the addressee, who doesn't yet exist, but that is where the signature starts ... When I sign for the first time, that means that I am writing something that I know will have been signed only if the addressees come to countersign it. Thus the temporality of the signature is always this future perfect that naturally politicizes the work, gives it over to someone else, that is to say, to society, to an institution, to the possibility of the signature. (Wills 1994, 19)

This idea of the artist refers more to his practice as craftsman than to the idealistic tradition of the genius. It is a re-reading of the history of art beyond the dominating perspective of subjectivity, which Heidegger, as one of the first, criticized in $\mathrm{Be}$ ing and Time and The Origin of the Work of Art as a false inversion of the ontological relation. The distinction between 'being at hand' (Vorhandenheit) as existence and 'being to hand' (Zuhandenheit) as utility led Heidegger to the very important assumption that we have no real relation or contact with being as being, but that we can only relate to it by transforming it into 'Zeug' (from 'ziehen': to pull: draft in the sense of the trait). This constitutes an implicit re-evaluation of the hand as the primordial or basic medium of data processing. Our relationship to the world is literally mediated by the hand that tears up the things near us and makes them useful. However, in this way, things are only given when they are framed by utility and as data of perception; they do not exist in themselves.

Contrary to all utilitarian dealings with things as 'Zeug', the mind is confronted with what Heidegger calls the 'Zeigzeug': the toolkit, a reference to what turns 
out, at the moment of the accident to be the annihilation of all readiness or handiness, the withdrawal of the things themselves: to be not at hand, to refuse all utility and to be nevertheless there only as a useless obstacle. In this sense, things are reduced to their pure and mere being-there, their Vorhandenheit, their being at hand, which, strictly speaking, does not at all stand in relation to any hand or handiness. They do not speak to the hand, but to the eye. We are dealing here with the very important occidental difference between the visual and the haptical (a seeing by touching, as when we say that blind men see with their hands). And it is no wonder that this dimension was largely developed in the new technical media, such as photography, where the artist's hand is substituted by a machine, by the so-called apparatus, with whose help 'Nature' inscribes itself as 'Pencil of Nature' (Talbot):

This most exact technique can give the presentation a magical value that a painted picture can never possess for us.... [T] he viewer feels an irresistible compulsion to seek the tiny spark of accident, the here and now. (Benjamin 1980, 202)

But this alienation as distance changes to a transcendental condition of magic, called aura: the aura of authenticity as absence or distance. The aura, as Benjamin argues, is the opposite of the trait, the track as presence:

What is aura? A strange web of time and space: the unique appearance of a distance, however close at hand. On a summer noon, resting, to follow the line of a mountain range on the horizon or a twig which throws its shadow on the observer, until the moment or hour begins to be a part of its appearance - that is to breathe the aura of these mountains, that twig. Now to bring themselves closer - and closer to the masses - is as passionate a contemporary trend as is the conquest of unique things in every situation by their reproduction. Day by day the need becomes greater to take possession of the object - from the closest proximity - in an image and the reproduction of an image. The removal of the object from its shell, the fragmentation of the aura, is the signature of a perception whose sensitivity for similarity has so grown that by means of reproduction it defeats even the unique. (Benjamin 1980, 209)

Derrida was aware of the problematic way in which this ideology of reproduction tried to forget the difference between the trait as witness (as high fidelity) and the artificiality of the made, which Derrida named, in the very context of media studies, artefactualité (after artificial and factual) and which leads to the question of the legitimization of the trace as mise en abyme of authority (Derrida 1996, 11). In Force of Law: The 'Mystical Foundation of Authority' Derrida refers to another text by Benjamin, entitled Critique of Violence. The basic figure of the possibility of justice is here referred to as a promise and repetition - a figure similar to iterability or the countersignature as re-establishing the original by means of the posteriority of visualization, reception, and representation: 
A foundation is a promise. Every position... permits and promises.... Thus it inscribes the possibility of repetition at the heart of the originary... Position is already iterability, a call for self-conserving repetition. Conservation in its turn refounds, so that it can conserve what it claims to found. (Derrida 1992, 38)

The founding sign appears as a given hand, a signature. But it is a performative and not an affirmative act: if we can say that the trace engenders its own origin without proving its legitimacy, the acting or practicing of justice in its own singularity (without generalization) generates authority without decidability, guarantee, or calculability. This double bind or double mark of the mystical ground as the secret of innocence and transgression introduces the bymen of authority, the veil of visibility that cannot be torn without destroying the founding tension of intention as a move towards a forthcoming alteration instead of towards a future as reduplication of the present.

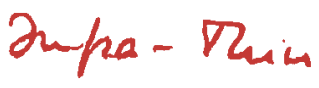

We are also dealing here with a narrative of touch, a narrative of membranes as revived in the well-known anecdote of Balzac's fear that photography may flay the skin. This of course has its roots in Lucretius and late antiquity's theory of eidola, which lives on in the mythical theme of photography as the removed skin of things. It also refers to a narrative of the injured gaze, of scarring, stitching, or the 'suture' mending the gaping hole between the original and the image. The aspects of skin under discussion here concern not only the skin on the surface of objects, but also the sensitive skin of that which touches. With Derrida we can connect this back to the origin of painting, to the story of Dibutade, the daughter of the Corinthian potter, who records the silhouette of her lover on the wall with a stick before he goes off to war:

Unlike the spoken or written sign, it does not cut itself off from the desiring body of the person who traces or from the immediately perceived image of the other. It is of course still an image which is traced at the tip of the wand, but an image that is not completely separated from the person it represents; what the drawing draws is almost present in person in his shadow. The distance from the shadow or from the wand is almost nothing. She who traces, holding, handling, now the wand, is very close to touching what is very close to being the other itself, close by a minute difference; that small difference - visibility, spacing, death - is undoubtedly the origin of the sign and the breaking of immediacy; but it is in reducing it as much as possible that one marks the contours of signification. One thinks the sign beginning from its limit, which belongs neither to nature nor to convention. (Derrida 1976, 234)

The touch, then, lies between denotation and connotation. The trace inscribes itself in the course of a development that includes all three aspects: touch, imprint, 
and its becoming autonomous as an icon. This new autonomy comes about, to put it more precisely, by a process of montage, that is, by construction or mise en scène. It is, however, determined by an implicit double bind, which deconstruction can again make explicit: the double bind between origin and invention. Derrida's designation of this minimal gap as différance has a predecessor in Marcel Duchamp's concept of inframince, which may be translated as 'wafer-thin' or 'infra-small', 'infra-thin' or 'infra-minimal' (as opposed to 'ultra-'):

This is one way in which the 'same' distances itself from itself, becomes dissimilar to itself, diverging from itself by an 'infra-minimal distance'. On the spatial level... Duchamp connects the question of the infra-minimal with the question of 'sameness' - that is, a way of tracking a 'same' back to a prototype, as in serial fabrication, which served as Duchamp's model. (Didi-Huberman 1999, from the German translation of the French original, 173)

Duchamp had already demonstrated this type of shift in the repetition or difference of identity in his ready-mades, which ushered into the artistic arena not only the industrial principle of pre-fabricated products, but also that of the serial production of apparently identical stereotypes. The point, then, about ready-mades such as the Bottle Rack (1914) or the Fountain (1917) that Duchamp apparently found in a department store - is that in the process of aesthetic presentation, individual examples depart from a context which determines them as a whole unit, so that they become totality in themselves, without any reference to what they represent, like an object identical to its image. Thereby, it suggests the absence of an original prototype or object. And here we arrive at the question of the occult, of the cryptic element of the mediality of that which represents, or rather - remembering the minuteness, the imperceptible difference of the gap posited by Duchamp - of the crypticness of infra-mediality.

But what is meant by the concept of infra-mediality? One might say it is an answer to the question of the monadological element of the translatability of data in the sense of their shifting from the authenticity of the drawn trait to the manipulative re-appropriation as authorship. Our attention is now focused not on intermediality's horizontal shifts, but on the vertical compression of heterogeneity; that is, on the relocation of the moments of intermediality - such as 'fault-lines, gaps, intervals or interstices, as well as borders and thresholds, in which their media differential plays a part' - to the interior of a structure with its layerings, dislocations and thresholds: as the potentiality or intensity of an inner, infrastructural change (Paech 1998, 25). According to Leibniz, monads have no windows, which does not, however, mean that they cannot form constellations or represent them. They must do this as monads, i.e., as fragments of a whole that develops (further) within these fragments, autonomously but at the same time synchronously or affinitively. What counts is the media auto-referentiality of the semantics that attains meaning by means of the potentiation of the system.

Infra in this sense, then, implies more than simply the immanent or internal. Rather, it implies an internal inclusion, subcutaneous virtualities, something con- 
cealed underneath that does not, however, pre-exist as anything substantial but reveals itself - like infra-red energy - in its subsequential effects. The use of the term in Duchamp's phrase infra-mince can be applied to Plato's chora as well as to Derrida's différance. The poem of the same title ('If tobacco smoke smells of the mouth it issued from, both scents are married in infra-mince') celebrates this definition of the transition from the possible to the nascent or the difference 'between two serially produced objects (from the same mold)' (Tono 1984, 55). Infra-mediality, then, has to do with the infrastructure of the media as a virtual dispositivity of the medium, the inner work: as an internal act of translation, as a version taking all things into account, as it were. One might also - with reference to Benjamin's essay 'The Task of the Translator' - speak of an infra-linear version which, like Benjamin's 'interlinear version' between the original and the translation, sees itself as a virtual and transcendental subtext, functioning 'underneath' or 'inside' ('infra'), representing something like a dispositive or invisible/unconscious 'matrix' for its intention - but as a symbol for the unconscious of the trait. ${ }^{I}$ Intention as a kind of inner exterior now turns out, in fact, to be an infrastructure - certainly in terms of Benjamin's original phrase, 'in the intention of the asserted', where 'in' indicates how the determination of the kind of assertion occurs 'inside'. ${ }^{2}$ And it is in this sense that Duchamp himself speaks of creation as 'osmosis', 'a transparence from the artist to the spectator... taking place through the inert matter'.

'Intention' as a metaphor could not only replace the tiresome division of index vs. icon, but could also perform the role of missing link between the three aspects of materiality, apparatus and code. One should emphasize the critical valency of this reminder of 'artifactuality', obligated to the subconscious, as well as recognizing it as a new impetus for research: inframediality inquires after every medium's intentions with regard to other media in its 'interlinear version' (Benjamin). The infralinear version, however, reduces each particular mediality by its own computations and representations of, for example, visual knowledge - through the paper or the screen, for instance, as a kind of 'approximate remembrance' of the real, which clears a path for its own memory in the narrativity of the allegorical aspects of the material recording.

The trace or trait thus exhibits its own genealogy, the implements hidden in the actual infra-trait. Actually, what we call 'authentic reproduction' is projection, because the authentic can only become visible as trace of the 'producing' hand in its reproduction and afterwards. Thus we arrive at the final thesis: we cannot close the gap between the drawing and the drawn, but need to stay within the chain of supplementations, as in a film where the next picture sutures the gap of the one before. This whole play of difference, delay, and deviation constitutes the artistic production and the responsibility of the artist as author: the difference between the piece of work in its total materiality and the artist as its first spectator who 'smells' of what he has done in this afterglow of the infra-thin transformation.

The authenticity of handwriting appears precisely as a re-appropriation of this gap. Thus, authenticity does not reveal a reference to a real or ontological origin. Rather, it appears as an intention in the sense of a drift of difference. The so-called 'infra-thin signature' disseminates each trait in a kind of performativity of produc- 
58

Sign Here! / The Authority of Drawing

timon. The act of signing then resembles less a giving hand in the sense of a source of originality that the hand owns and now gives away. Instead, this metaphor of gift or present transforms into the slightly different iconography of the presence of a touching hand, living on the borderline of the seeking and of the meeting impulse of drawing.

Qichoul Clot

SIGN HERE! 
Notes

1. Compare with Lyotard, Jean-François, Discours, Figure. 1971, 339.

2. This phrase, literally Intention vom Gemeinten (from: Benjamin, Walter.

'Die Aufgabe des Übersetzers', in:

R. Tiedemann and H. Schweppenhäuser (eds.), Gesammelte Schriften, vol. 1,
$1974,14)$ is translated into English as 'the intended object' (Benjamin, Walter. 'The Task of the Translator: An Introduction to the Translation of Baudelaire's "Tableaux parisiens"', in: Illuminations, 74).

\section{Works Cited}

Benjamin, Walter. 'Die Aufgabe des Übersetzers', in: R. Tiedemann and H. Schweppenhäuser (eds.), Gesammelte Schriften, vol. 1. Frankfurt am Main, Suhrkamp1974. (English translation: Benjamin, Walter. 'The Task of the Translator: An Introduction to the Translation of Baudelaire's “Tableaux parisiens”', in: Hannah Arendt (ed. and intro.), Illuminations.

Trans. by Harry Zohn. New York: Schocken, 1968.)

- 'A short story of photography', in: A. Trachtenberg (ed.), Classic Essays on Photography. New Haven: Leet's Island Books, 1980.

Derrida, Jacques. Of Grammatology. Trans. and with an introduction by Gayatri Chakravorty Spivak. Baltimore: Johns Hopkins University Press, 1976.

- Psyche. Inventions de l'autre. Paris: Éditions Galilée, 1987.

- 'Force of Law: The "Mystical Foundation of Authority"', in: D. Cornell, M. Rosenfeld, and D. Carlson (eds.), Deconstruction and the Possibility of Justice. New York: Routledge, 1992.

- Memoirs of the Blind: The Self-Portrait and Other Ruins. Trans. by P.A. Brault and M. Naas. Chicago: University of Chicago Press, 1993.

- 'Artefactualités', in: Derrida, Jacques and Bernard Stiegler (eds.), Echographies de la télévision. Entretiens filmés. Paris: Institut national de l'audiovisuel, 1996.

Didi-Huberman, Georges. Ähnlichkeit und Berührung. Archäologie, Anachronismus und Modernität des Abdrucks. Köln: DuMont, 1999. (German trans. of: Didi-Huberman, Georges, L'Empreinte. Paris: Editions du Centre Pompidou, 1997).

Heidegger, Martin.On Time and Being. Trans. Joas Stambaugh. Chicago: University of Chicago Press, 2002.

- 'Der Ursprung des Kunstwerks', in: Holzwege. Frankfurt am Main: Klostermann, 1952.

Kant, Immanuel, 'Über das Misslingen aller philosophischen Versuche in der Theodizee', in: W. Weischedel (ed.), Werke in zwölf Bänden. Bd. XI. Frankfurt am Main: Suhrkamp, 1964.

Lyotard, Jean-François, Discours. Figure. Paris: Klinckseick, 1971.

Paech, Joachim. 'Mediales Differential und transformative Figurationen', in: J. Helbig (ed.), Intermedialität. Berlin: Erich Schmidt, 1998.

Tono, Yoshiaki. 'Duchamp und "Inframince"' in: Duchamp. Köln: Museum Ludwig, 1984.

Trilling, Lionel. Sincerity and Authenticity. London: Oxford University Press, 1972.

Wills, David. 'The Spatial Arts: An Interview with Jacques Derrida', in: Peter Brunette and David Wills (eds.), Deconstruction and the Visual Arts. Cambridge: Cambridge University Press, 1994. 


\section{Authenticity and Objectivity in Scientific}

\section{Communication:}

\section{Implications of}
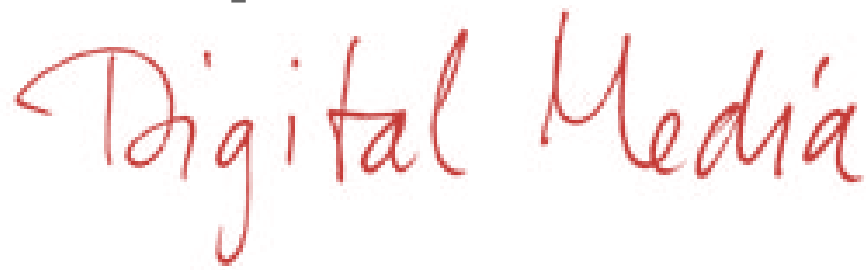

Introduction

Digital media are quite unlike the 'mechanical' reproduction media that figure so often in the literature of cultural and media studies. Both the interaction between author and user through the digital medium, and a number of other intrinsic qualities of many digital media, lead to novel interpretations of the concepts of 'copy' and 'authenticity' in the digital world. Digital media re-introduce some aspects of authenticity that were lost through the use of purely mechanical media. Digital media also lead to new conceptions of authenticity and power, related to shifts of control from author to reader and from publisher to author, and a shift from mechanical production to digital reproduction. In addition, digital media result in a reduction of commonality and shared reading experiences, and a re-definition of the concept of authenticity in terms of readership rather than authorship. These are issues that are of growing importance for scientific communication that is becoming almost totally dependent on digital media.

Scientific communication in the modern sense implies the distribution of multiple copies of an author's work, preferably on a global scale, although generally confined to a specific disciplinary field. It is one of the requirements of the scientific communication system that these copies are 'authentic', i.e., exact copies of a certified original, and that they remain authentic over time. This is not easy in a 
world where digital information can be manipulated with ease, and where digital documents are inherently fluid and unstable. In addition, scientific information is required to be 'objective' and therefore to abstain from devices that enhance selfexpression rather than content. As we shall see, digital media offer both authors and readers various opportunities for self-expression in the communicative act. Since 'authenticity' may refer to an original, but also to originality, the concept of authenticity becomes highly problematic in the context of digital media.

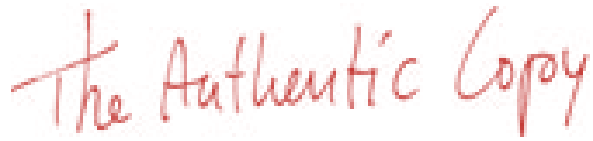

What do we mean by an 'authentic copy'? In the traditional world of print publication, the word 'copy' usually refers to an abstraction: the original text as established by the publisher. This is not necessarily the author's original text (often referred to as 'manuscript' even when it is not written by hand), and in fact it usually is not. As a result of the editorial, certification, and publication processes, the original text (i.e., the submitted manuscript) is changed, often quite extensively. In form and even in content, the publisher (or the publisher's proxy, the editor) has the final say. In this case, 'authenticity' refers to the original published text, not to the original text that the author has submitted. The distinction between author and publisher is therefore relevant with regard to authenticity. Within the scientific community, in the context of legal issues such as copyright, and for the practice of archiving, it is always the publication (rather than the manuscript or pre-print) that is considered to be the authentic work.

Written communication can be regarded as a sequential process in which, as far as publications are concerned, various actors perform specific functions. ${ }^{I}$ In its simplest form, this 'information chain' consists of the sequence Author-PublisherReader (Illustration 1). ${ }^{2}$ Each of these actors exercises some form of control over form and content. But to a large extent it is the publisher who is in control.

Auther

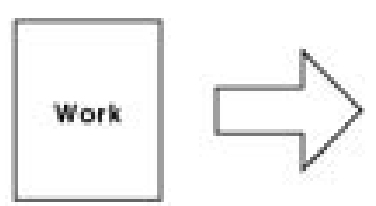

Publisher

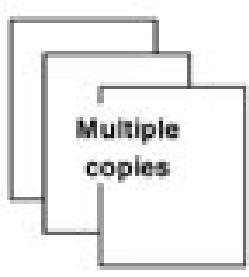

Distribution over time and spuce
Reader
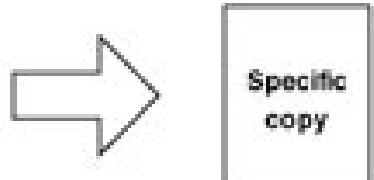
The locus of control regarding authenticity has been with the publisher for a considerable period of time, in fact it has been there since the invention of the printing press. But this is now shifting in two directions as a result of the introduction of digital media: a shift back from publishers to authors, but also a shift from authorship to publishing.

Many publishers now require authors to produce camera-ready copy. This gives authors more control over the final publication. However, this is offset by publishers' 'editorial policies' that put strict requirements on the format to be used by the author. A more conspicuous shift towards author-control is self-publishing (Harnad 1995). Digital media give authors the opportunity to publish their works themselves, usually via the Internet, giving them almost total control over form and content (as well as over copyright - the prime motivation for Harnad's 'subversive proposal').

However, there is also a shift in the opposite direction. Digital media allow readers (or 'users') much more freedom to define the visual characteristics of what they read. They also allow users to create their own, highly individual paths through digital content resources. This implies that users not only have more control over form and structure, but also, in a pragmatic sense, over content. By selecting and linking fragments of information, the user virtually creates his or her own work. The authors - if they exist at all - become mere suppliers of semantic components to the user.

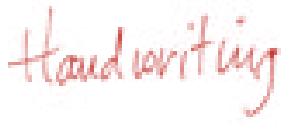

The history of media has often been described in terms of major and minor revolutions. The idea behind such a revolution is that it introduces a new medium and a new communicative practice that emphasizes the characteristics of the new medium, and has little use for the characteristics of the previous one. It is true that in the transition from one medium to another, a certain amount of remediation takes place. But eventually the old medium disappears as a technological artifact, and with it many of the cultural practices, forms and genres that surrounded it disappear as well. There is nothing new in this. The handwritten medium and the Latin language were effectively marginalized by the 20 million books produced by the printing press between 1450 and 1500 (Febvre and Martin 1958; see also Birkerts 1994). Print also destroyed the culture of orality: the practice of reading aloud to oneself and to others was replaced by silent reading.

A later example is the 'revolution' caused by the introduction of the keyboard as a medium for writing. For many, the keyboard has not only destroyed the specific characteristics of handwriting, but even the ability of handwriting. ${ }^{3}$ Now that the mechanical keyboard (typewriter) has been replaced by the digital keyboard (computer), we have moved even further away from handwriting. 


\section{Autograpily and Auttentiaty}

If the typewriter destroys handwriting, it might be thought that the computer must destroy even more. What, in fact, is being destroyed, is authenticity in its most immediate form, and in a sense is very different from that described above in the context of scientific communication. Autography's claim for authenticity (as opposed to typed writing) is based on a conception of handwriting as 'an un-(ex)changeable, unique and authentic "signature" that claims to guarantee the presence of an individual writer during an historically unique moment of writing'. ${ }^{4}$ In contrast, typed writing would have to be characterized as allographic, i.e., iterative and reproducible. The argument, then, is that with handwriting the medium does not only convey information, but also a physical token of identity as an authentic and recognizable expression of the writer as an individual, and that this form of authenticity is destroyed by the mechanization involved in typed writing.

However, handwriting has no claim to this type of authenticity per se. Throughout the ages, the culture and practice of handwriting has been based on standardization and the elimination of personal characteristics rather than idiosyncrasy. In mediaeval manuscripts it is often very difficult to distinguish between the hand of different scribes. Handwriting as learned in the classroom has always been highly standardized. Even nowadays the educational goal remains standardized, 'legible' forms of handwriting, and the Internet offers many solutions for achieving this goal, including computerized, interactive learning aids. 5 Deviations from the standard are commonly described as 'bad' handwriting. ${ }^{6}$

This standardization is quite reasonable and understandable. At the end of the day, writing has a communicative function. Communication theory tells us that it benefits from the use of standardized encodings that are shared by both communicator and receiver. The 'authenticity' of handwriting therefore either stems from a person's inability to comply with the accepted standard (i.e., a lack of communicative skills), or the writer's wish to make a specific statement by not playing the game in the prescribed way. Enter the artist's dilemma: authentic communication requires breaking the conventional rules of communication.

The conclusion for scientific communication is clear. The scientist is not an artist, and the expression of his or her personality is not part of the game. The shift from handwriting to standardized, mechanized writing is but one of the dimensions of the shift towards objectivity in scientific communication over the centuries (Gross et al. 2002).7 For scientific communication, then, the question is whether digital media sustain the required objectivity, or regress towards increasing subjectivity.

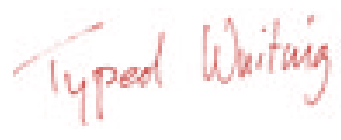

Speed, standardization, and reproducibility are three characteristics of typed writing that are perceived as advantages over handwriting. Standardization guarantees a high degree of legibility and therefore supports the communicative function. Reproducibility in the context of typewriting means two things. First, however often 
and by whomever a text is typed, it will always lead to more or less the same typographical result. ${ }^{8}$ But second, and perhaps more importantly, the typewriter offers the possibility of auto-reproduction: the 'carbon copy'. As such the typewriter is not really different from the printing press or even the scriptorium (as a highly standardized human copying machine). But what might be relevant as a phenomenon in the development of culture is that for the first time the typewriter allows for communication and reproduction of content without the intermediary form of handwriting: the author expresses ideas directly by means of the typewriter. ${ }^{9}$ There is no handwritten original, and this will become especially relevant in the context of digital media. Whereas with typewriting there still remains a physical object that could be referred to as the authentic original, that is no longer the case when a text resides solely in a computer's memory.

If we regard handwriting as a culturally significant phenomenon, then we can argue that the author relinquishes his or her authenticity by submitting to the conveniences of the typewriter: the written object might as well have been created by somebody else. And as far as the medium of writing influences the form and even the meaning of writing, the shift from handwriting to typewriting (and further to keyboard writing) is at the very least significant. ${ }^{10}$

It can be argued, however, that typewriting as a standardized form actually safeguards authenticity to a much higher degree than handwriting. This argument requires a different approach to the concept of 'authenticity'. At the level of connotation, of conveying meaning, handwriting is problematic. The more individual (i.e., 'authentic') the handwriting, the greater the danger is that the reader will misread, and therefore misinterpret the meaning of the author as expressed through the handwriting. As noted above, this communicative problem of handwriting is increased in the context of the 'artist's dilemma'. In other words, the authenticity of the text (its intended meaning) is undermined by the authenticity of the handwriting. In this sense, the typewriter and other systems of mechanical reproduction, including the digital keyboard, offers a greater chance than handwriting that the authentic meaning of the author will be communicated to the reader.

To a certain extent this paradox would seem to be merely semantic, involving two conceptions of 'authenticity':

- Type 1: authenticity with reference to identity ('that's me')

- Type 2: authenticity with reference to connotation ('that's what I mean').

However, the problem is more intricate, and there is a continuing conflict between the internal authenticity of meaning and the external authenticity of signature. The enormous success of mechanical and digital media might indicate that most people find the former more important than the latter. Our rational Western culture values semantic meaning more than the mode of expression. Yet the preference of some people for handwriting in the face of increasing pressure to adopt standardized, mechanical, and networked communication media, might point to the existence of two distinct psychological types (i.e., external/visual/subjective/ artist versus internal/mental/objective/scientist). 


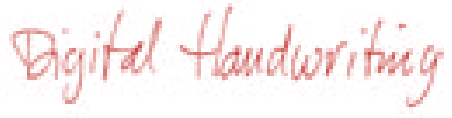

The expression of identity is related to the expressiveness of the medium. The handwriting author has no more than pen and paper to manifest his/her identity. The digital author, on the other hand, has a vast array of means to create a personal signature in the visual construction of the text. ${ }^{I I}$ Here there certainly is an advantage over the typewriter, if not over handwriting.

The main difference lies in the design and parameterization of the signature. Design implies that the visual expression of content on paper or screen is governed by the intentional application of a schema or format that has been created consciously beforehand. In the digital context, this type of design is highly parameterized: it consists of a finite set of attribute/value pairs that determine specific characteristics of the visual appearance of the text. Superimposed on the basic design are the local choices of the writer to vary the parameters by altering their values. So the writer can choose an initial template or style sheet: a schema of layout elements such as font family, size, color, etc. In writing, the author then may or may not deviate from this schema, e.g., by altering the font, size or by using bold or italics to express emphasis.

The typographical variety available in digital typed writing is therefore not necessarily less than that available in handwriting. The main difference is that handwriting uses visual elements as a form of immediate, graphical expression, whereas in digital writing expression is governed by the ability of the writer to command the machine, i.e., to control the application of typographical parameters. In this sense, ergodic reading is supplemented by ergodic writing (Aarseth 1997). As everyone using digital writing tools knows, the flow of thought is continually interrupted by the need (or desire) to control layout and typographical parameters and in general be involved - at least to a certain degree - with the visual characteristics of the text. ${ }^{22}$

Now, one might argue that this is all very well, but that the essence of handwriting has to be found not in typographical elements (e.g., layout, size, color, etc.), but in the letter formation itself. The mechanical or digital writer has to choose from a finite set of prefabricated, industrial fonts, whereas handwriting makes use of highly individual, personal letter formations (Ong 1982). However, the borderline between handwritten letters and industrialized fonts has become more fluid. To begin with, there are now methods to create a personal font based on a person's own handwriting. On the Internet, one can find commercial services that offer to create a personal font based on an example of the client's handwriting. Personal this may be, but it does remain a font in the traditional sense: a fixed set of tokens, where every ' $a$ ' is identical to every other ' $a$ '. However, recent rule-based technologies now allow for a seemingly endless variation of letter forms within a personalized font. ${ }^{13}$ Digital media are re-creating the illusion of personal handwriting while retaining all of the other characteristics of digital writing, including reproducibility and parameter control. One could argue about how echt this form of digital handwriting is. But it is perhaps at least relevant to note that there is apparently a need (and therefore a market) in the digital world for something that 
will at least provide the illusion of personal handwriting. A more recent example of the reverse is a form of digital handwriting recognition that is not based on standardized tokens imposed on the writer (as in most personal digital assistants), but that recognizes individual handwriting. ${ }^{I 4}$

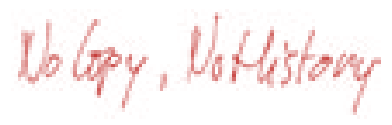

Our thinking about information and media is to a large extent based on the wellknown conduit model that describes information as flowing over a system consisting of at least a sender/origin, a medium/channel and a receiver/destination (Reddy 1993; Day 2001, chapter 3). This model implies, amongst many other things, authorship and authenticity, i.e., that a message as received should be identical to the message sent. ${ }^{15}$ Authenticity therefore also implies that the message must be stable over time (i.e., between sending and receiving). ${ }^{I 6}$ That this is not always the case, is perceived as a communications problem to be resolved by perfecting the communications system. But in the digital world, things can be different. What if authorship and authenticity are intentionally left out?

Much information is generated by processes and systems (e.g., sensors, surveillance cameras). It is difficult to understand who or what the author is in these cases. The concept of 'meaning' in the absence of an author is problematic, as is 'authenticity'. But stability over time can be even more contentious. Within the prevailing concept of a communications system, information is always by definition old information: images of history, of what has been. It pertains to something from the past that is being conveyed over time and space to the reader. However, digital media allow the communication of real-time information that pertains to the exact moment that it is received. A moment later the information is no longer valid, and it has to be refreshed. Here, authenticity depends on immediacy and can be characterized as 'zero-duration authenticity'.

Even traditional media such as the newspaper move in this direction once they go online. Shayla Thiel has devoted an interesting discussion to online newspapers in which she describes the medium as an experience rather than as a product (Thiel 1998). The online newspaper changes from moment to moment, and is also highly personalized. ${ }^{I 7}$ Not only traditional documentary characteristics such as quality markers and context indicators disappear, but the entire concept of 'authenticity' has shifted from the author to the reader. There is no longer an authentic expression of the writer as an individual. What counts is the reader's feeling of authenticity: whether the newspaper (or any other digital format) provides an authentic experience and gives the reader the illusion of active involvement in the dynamics of the world around him. But, as Thiel observes, the online newspaper is 'more concerned with look and feel than with getting the best news and information'. Thiel is skeptical about this development towards ephemeral media, but she also argues that it is well adapted to the postmodern culture of our times, and to future generations of readers. 
However this may be, the example shows that with highly dynamic digital media, there is no author, no original, no copy, no authenticity, nothing to preserve, and therefore no history. It is precisely these characteristics that prohibit such media from playing a role in the world of objective knowledge and scientific communication.

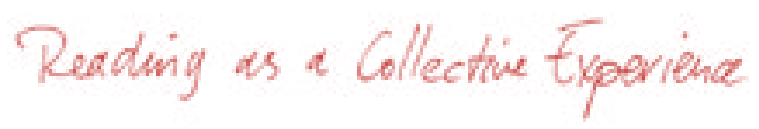

The lack of fixity has more significance than a mere lack of history - if there is nothing to record, who cares about history? However, it also has profound implications for the role of information in providing a cultural context for communication and in creating collective experiences and group identities. To explain this, we must return to handwriting. In terms of communication, from the reader's viewpoint, handwriting is personal in the sense that it is directed towards the intended reader or at least as a member of a limited group of readers. The direct communicative characteristic of handwriting stems to a large degree from the fact that the author usually knows the reader(s), and intentionally communicates with him/her/ them through the medium of handwriting. Typed writing on the other hand, because of its potentially allocutive character, can be directed at a larger group of anonymous readers. Handwriting resembles conversation, whereas typed writing resembles declamation. Handwriting emulates the body language and tacit knowledge sharing of direct interpersonal interaction, whereas typed writing has to revert to rhetorical techniques in order to achieve communicative results.

One of the most fundamental (though often neglected) consequences of typed writing and other allocutive media, including print, is the creation of shared reading experiences amongst groups of people. In the context of handwriting, people can (or have to) tell each other about what has been written to each of them individually. In the context of typed writing, people can discuss shared content (knowing, or at least, assuming that they have all received the same information), or, perhaps unconsciously, act and cooperate on the basis of shared information. Scientific communication is based on this concept of shared reading experiences, assuming access to, if not familiarity with, the shared body of knowledge stored in the 'canonical archive' of science and embodied in scientific texts through a network of references (Rowland 1997). Shared knowledge and - more generally - shared symbolic materials, are the basis of any cultural community and identity, and even of the definition of the 'self' as individuality with reference to a common ground. Thompson (1995) describes the shift from 'local knowledge' acquired in the context of face-to-face interaction to a process of self-formation dependent on access to mediated forms of communication. As long as there exists a relatively limited repertoire of symbolic materials, this shift need not necessarily destroy the common ground for human interaction and coexistence. To a certain extent the rise of mediated forms of communication and informing has widened the common ground to facilitate interaction between individuals and groups who formerly might not have had anything to share. But the culturally significant switch from in- 
dividual to shared knowledge that was brought about by the gradual move from handwriting to typed writing is now being reversed through digital media: reading is becoming a more individual and less collective experience. The disappearance of collective reading experiences, and therefore the elimination of shared contexts, is problematic to say the least. Imagine the difference between a classroom (or board meeting) where all of the attendants have read the same materials with one where all of the attendants have read entirely different materials - not once but for most of their lives.

There are various reasons why this shift towards individualization of reading experiences is happening. One is the use of highly adaptive, interactive, and hypertextual digital media. This means that the information presented to the reader, and the path traversed by the reader through the available information, is highly individual and contextualized. This is not only true in the sense that the reader constructs a reading experience from loosely linked fragments, but also in the way that literary or scientific texts can be traversed once they are available in hypertext form (Svedjedal 2000). Another reason is the availability to the user of enormous amounts of information on any topic, diminishing the statistical likelihood that two people will obtain the same information.

With regard to authenticity, it could be argued that where there is no common ground, every utterance and every experience is 'authentic'. But when everything is authentic, the whole concept loses its meaning. If digital media do indeed destroy authenticity, it is precisely because they destroy commonality. The elimination of shared contexts is one of the most problematic effects of digital media. What it will mean for communication in general, and for self-perception, group identity and even the concept of distinct 'cultures' remains to be seen. When background knowledge cannot be assumed, and when even a reference (e.g., a hyperlink) need not necessarily imply that the reader will encounter the same information as the author, the ideal of objective knowledge might break down, and knowledge will mean little else than what one happens to know.

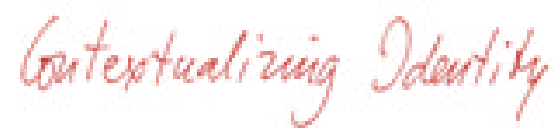

Since the very beginning of printing, there has been a tension between the author and publisher with regards to identity. For the author, the work published is his or her work, and should be recognizable as such. The publisher of the 16th century, however, had little concern for the author, whom he would regard as a mere supplier of raw material. The published text was the publisher's work, and the transfer of copyright from author to publisher that is still common today underlines that in this respect little has changed since the 16 th century.

It is important to the publisher that his work be recognizable, i.e., that it has an identity, and that it can be seen as an authentic work of the publisher. This underlines the fact that, ultimately, a publication is a product rather than a 'work'. The producer seeks a certain profit on any product over the cost of developing, making and selling it. Within these financial constraints, the producer has some degree of 
freedom to vary the ergonomic and aesthetic characteristics of the product. These characteristics often distinguish one product from another, i.e., they define the product's identity (as opposed to its functionality), and by proxy, the producer's identity as well. For the products of a publisher, the aesthetic identity is determined by factors including typography, page design, and citation style. We all recognize a Penguin when we see one, and a doctor will not mistake Nature for the Lancet.

The commercial importance of product identity is one of the reasons why publishers (and their predecessors, the printers) have taken over the locus of control over what constitutes the authentic work. They were able to do so because the copy ultimately has a far greater communicative and economic significance than the original. The singular authenticity of the original had to lose out against the multiplicity and authority of the copy. ${ }^{I 8}$ But at the same time the control invested in the publisher resulted in a tightly organized system of certification, distribution, and archiving that created a Popperian 'World 3' of objective scientific knowledge. Without this center of control, scientific information would have remained 'authentic' in that other sense: the subjective expression of the individual author. Scientific knowledge as we know it would not exist, there would be only scientific opinion.

However, it has already been indicated that the locus of control is now also partly shifting towards the user. ICT solutions allow the user a certain degree of freedom to vary the visual parameters such as font type and size, line length, color, inclusion/exclusion of images, and even style of headings, citation style, link style, etc. This subverts the producer's attempts to force his own aesthetics and identity on the reader. ${ }^{I 9}$ At this stage, we are far off from the authentic handwriting of the author, who has no way of even knowing what his text will look like when it reaches the reader.

On the other hand, the author in the digital world has far more opportunities for the creation of an informational identity than in the earlier eras of print or handwriting. One example is self-publishing on the Internet, which puts the author in control, even though he is still bound to numerous conventions - from the rules of scholarship to the prevailing standards and formats on the Internet. What in fact is happening is that the 'identity' of the author is becoming bound to his or her network presence: 'I know you from your home page'. If we regard the visual expression of network presence as a form of virtual handwriting, then this is indeed a complex concept. It could even include the perceivable links to other authors as a network of mental relationships: 'I am whom I link to'. ${ }^{20}$

In this way, authenticity becomes a matter of contextualization. It is not the text and its form of expression, nor the author's signature that defines authenticity. The author's 'signature' emerges from the context into which the text is embedded, the relationships with other texts and individuals and groups. On the other hand, the reader creates an authentic reading experience through exactly the same process of contextualization, by interactively linking objects in information space to create the unique, personal work that shapes his identity and that nobody else will have access to. 


\section{Digital Futhentiality}

The use of digital media in contexts where information is used to create permanent records has resulted in a great deal of discussion about the concept of 'authenticity' amongst information professionals. For archivists, an 'authentic' document is either the original, unmutated document, or an exact and certified copy. For the author, a document is the expression of his or her ideas, opinions, and beliefs. It is authentic precisely because it relates to his or her identity as an author. That is what modern authorship is about, and why works are not published anonymously. It also explains why plagiarism produces highly emotional reactions and severe penalties: it is not about stealing information, but about negating the author's identity.

But if a document is a personal expression of its author, he may wish it to alter it as he himself changes, otherwise it would not be him/her any more. One of the conspicuous characteristics of self-publishing by scholars and scientists is that they tend to continuously revise their 'publications'. The 'authentic' document is therefore the one that reflects the author's current ideas, opinions, and beliefs, not those he once had and now might want to distance himself from. ${ }^{2 I}$

These considerations allow us to introduce two further connotations of 'authenticity' that are applicable in the fluidity of the digital environment:

- Type 3: original authenticity ('that was me then; it's not me anymore')

- Type 4: current authenticity ('that's me now').

A different way of looking at this is to regard mental work as an ongoing activity, i.e., as a process. Documents themselves can be seen as a reflection of this process, developing, and changing as the mental process unfolds. From this viewpoint there is no 'final' or 'authentic' version. However, one of the dogmas of scientific publishing is that a publication is an 'official record' to be entered into the 'canonical archive' of science. The shift in meaning of 'authenticity' as a result of the way writers make use of digital technology could therefore have a significant impact on the role of publications in the scientific process, and on the way we record and preserve scientific output.

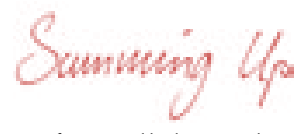

One conclusion that we can draw from all this is that the concept of 'authenticity' is complex and problematic. In fact, it is a multidimensional concept that can refer to:

- either the person (e.g., the author) or the object (e.g., the 'document') and its content

- either the author's 'identity' or his/her 'meaning'

- either the historical moment of creation or the 'here and now'.

We argue that handwriting is an expression of the author's identity at the moment of creation, whereas a continually updated digital document would reflect the author's current authentic self. What is truly authentic is then a matter of purpose. 
Another observation we can make is that the concept of 'copy' is problematic in that it presupposes an 'original'. It could be argued that mechanical systems such as the typewriter produce nothing but copies (or, if one prefers, originals), whereas in a digital context it could even be said that copies do not exist, i.e., every user is looking at the same 'original'.22

At the same time, users have the option to re-create the original, i.e., to impose their own expression of 'identity' and even meaning on the document. They even have (and use) the opportunity to create their own, authentic reading experiences from fragmented materials floating around in cyberspace. This is a result of the shift of the locus of control from the author, via the editor/printer/publisher, to the user. Simultaneously, the author is regaining control over her own work back from the publisher, allowing him/her to use digital media in creating a virtual handwriting within the context of her network presence.

Another observation is related to the idea that the 'authenticity' of authorship has to be seen as the individual deviation from common backgrounds and shared standards. To the extent that digital media destroy this commonality and enforce the heterogeneity of information experiences, the idea of authenticity in this sense may lose its meaning.

Handwriting is the ultimate form of authentic expression through the use of graphical/symbolic devices to the extent that handwriting knows no copies, but only originals. The culture of handwriting has disappeared for two reasons. One is the act of and desire for multiplication and multiplicability that was served in timely fashion by the invention of the printing press (but existed long before that event in the mediaeval copy shops). Multiplication, and the use of technical and digital means that make multiplication possible, isolates both author and reader from the authentic expression that handwriting can be considered to be. That is the price one has to pay for reaching a wider audience than mere handwriting can address. Another reason is the objectivity required by the modern scientific enterprise, which can only be achieved through the centralized, standardized, and certified procedures developed over the centuries by scholarly publishers.

A third reason is the desire to have greater control over the communication process. This is a complex issue. It involves the use of standardized technical means to overcome shortcomings in the technique of handwriting. It also involves attempts to control what happens at the receiver end of the communication chain by preventing false, i.e., non-authentic interpretations. That this attempt is futile, at least in the digital world, is clearly demonstrated by the amount of control the 'end-user' has over layout, structure, sequence and relationships within the body of information available in the digital environment. In this sense, not only 'copy' and 'authenticity' have lost their meaning, but the entire concept of 'authorship' as well. To the extent that the digital medium is rapidly becoming the primary medium, authenticity has shifted from the author to the reader. Perhaps it is a characteristic of a consumer society that the authenticity of reading has taken preference over the authenticity of writing. The tragedy is that whoever wishes to return to the authenticity of handwriting and thus to authorship, must inevitably lose his readership. What we see, then, is a struggle for power between writer and reader: 
the writer attempting to create an authentic expression of ideas, opinions and feelings; the reader attempting to create his or her own, 'authentic' text from the digital resources available. What is clear is that 'authenticity' acquires entirely new meanings in a digital world that empowers authors and readers with more control over form, structure, and content than ever before.

These new forms of authenticity could easily diminish the objectifying role (in terms of certification, standardization, and archiving) of the publisher and might eventually reverse the trend towards objectivity that has characterized the development of scientific communication since the 17 th century. Increasing the authenticity of both writing and the reading experiences through digital devices can only result in a more subjective grounding of communication. However, science has no need for a 'unique and authentic signature' for its authors, and should be capable of resisting the temptations offered by the digital medium for regressing towards a more subjective mode. Preliminary empirical research shows that the specific characteristics of the digital medium that might move science from its objective stance towards more subjectivity are not yet reflected in the primary medium of research: the peer-reviewed scientific article (Mackenzie Owen, forthcoming). But there are many indications that scientific readers do create their own traversals through information space, especially when publications are heavily hyperlinked. It will be interesting to see how this will develop, and whether authors and readers will continue to accept objective and authoritative sources above new forms of digital 'authenticity'.

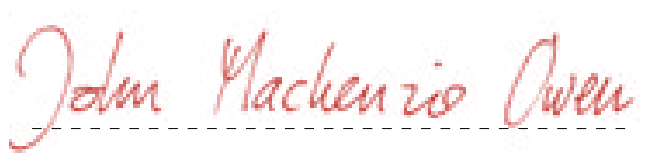

SIGN HERE! 
Notes

1. A publication is defined here as a text that has been made publicly available in any form, allowing undefined users to access its contents by any means.

2. See Duff 1998 and Mackenzie Owen and Van Halm 1989. The publisher can be defined as the actor responsible for creating and distributing multiple copies of the author's work.

3. Often expressed as 'typing has ruined my handwriting'.

4. Quoted from Sonja Neef, Handwriting as a cultural practice in the age of new media. Proposal to NWO.

5. See <http://www.handwritingforkids. com/handwrite $>$.

6. It is interesting to note that the revival of handwriting in the form of calligraphy (e.g., as practiced within the 'Arts and Crafts' movement of the late 19th century) is based on highly standardized historical examples - such as insular, Carolingian and Gothic scripts - for its letterforms. When calligraphy deviates from these historical forms, it often shifts towards a purely visual art form where communicating the original meaning of the text is of less importance, or of no significance at all.

7. Scientific communication serves to transform the subjective statements of the author into certified, objective information, and is, in the field of science, the mechanism behind the transition from 'subjective' to 'objective' knowledge as described by Popper (1972).

8. More or less: i.e., on the same machine and excluding typing errors and deviations in page layout.

9. Before the typewriter, printed texts were of course based on handwritten originals.

10. For instance, various contributors to Guntjahr 2001, argue that the visual representation of a text is a component of its meaning. See also Olson 1994. Ferris 2002 , even argues that the act of writing itself is transformed by digital media, becoming more like a conversational communication act.

11. Jerome McGann even argues that print's material (as opposed to handwriting's bodily) contingencies open up the text in the sense that it can acquire multiple and endlessly possible manifestations (McGann 1991). Digital media are, of course, no different in this respect. 12. Writing tools do exist that purport to free the author from such concerns. These mark-up languages such as TEX, LATEX and SGML are based on the concept of intentionality rather than control, e.g., the writer merely indicates the intention that a textual element should be a heading or should be emphasized. The 'program' then applies typographical rules to create the intended layout and typographical characteristics. Lyx (see www.lyx.org), an opensource word processor based on these principles, advertises itself as 'what you see is what you mean' rather than the WYSIWYG of standard word processors such as Word.

13. See < http://www.vletter.com/design_ visitor.htm > : To make a natural-looking handwriting style, multiple forms of each character need to be used. This is because the shape of each character depends upon the shape and type of connection of each surrounding character. vLetter calls this a contextual font. In addition, each font has a slightly different way of connecting each letter since each handwriting style is slightly different.

14. See <http://pi. parascript.com/piweb/ products/ritescript/rscr_prodinfo.asp $>$. The idea is similar to speech recognition systems. What is interesting is that the computer will understand a person's speech or handwriting, with the sole intent of transforming it immediately into computer code. 'For that's the stuff electronic texts are made of' (Kirschenbaum 2001). 15. This is what Weaver describes as the technical problem: the accuracy with which 'symbols of communication' can be transmitted, as distinct from the semantic problem related to the extent to which a message is correctly understood by the recipient (Weaver 1949).

16. In the context of written, printed, and digital (i.e., documentary) media, the time between sending and receiving, and therefore the required lifetime of the medium, can be extremely long, even spanning centuries. Stability over time is therefore a culturally significant requirement for any system of documentary communication. 17. Although even printed texts 'do not stay themselves', as Matthew Kirschenbaum argues, electronic information 'has a natural inclination to change, to grow and finally to disappear' (Kirschenbaum 2001). 18. For this is another reason for the power of the publisher, especially in scientific publishing: the publication (i.e., the copy rather than the original) obtains its authority from the fact that it is certified by the 
scientific community through the peer review process. The lack of certification is also the weakness of the authentic version as held by the author (Kling and McGim 1999; Rockwell and Siemens 2000), a weakness that is transferred to the digital pre-print repositories that are now multiplying under the 'open archives initiative' (see < http://www.openarchives.org/> and Michael Day 2001; Rusch-Feja 2002). A number of solutions have been proposed, e.g., involving readers in the peer review process (Arms 2002; Harnad 1996; Nentwich 1999; Roberts 1999; Weller 2000). 19. This explains why publishers prefer secure formats such as PDF above HTML. 20. This form of contextualization is becoming increasingly important in the digital environment. It relates to an aspect of 'collective identity' that is not specific to digital media, but that is greatly facilitated within a digital environment, by means of multiple (i.e., collective) authorship. Multiple authorship is the outcome of a number of trends, the most important of which is the growth of international collaborative research projects made necessary by the scale and cost of contemporary scientific problems, and facilitated by the global communication facilities of the Internet and the digitization of the research pro- cess itself, culminating in the emerging concept of e-science (see De Roure et al. 2003).

21. There are various technical solutions that facilitate this. One solution is to allow a document to have an embedded history, i.e., to make it consist of the accumulation of consecutive versions, although an author might not want to be associated with ideas that he or she no longer endorses. There exists versioning software that tracks differences between documents and re-creates a version on the basis of the original and a series of recorded differences. It is, of course, also possible to do this the other way round, i.e., to retain the latest version and to derive previous versions from it. Primitive forms of versioning are a standard functionality of word processors. Wagner and Graham 1997, describe the concept of versioning as an intrinsic functionality of digital documents. 22. A contrasting view is offered by Levy 1999 , who suggests that a digital document should be regarded as a 'mini-printing press' that sends copies of itself to anyone requesting it to do so. He also puts forward the interesting notion that documents are 'objects with the power of speech', things that we send out into the world to tell our story.

Works cited

Aarseth, Espen J. Cybertext: Perspectives on Ergodic Literature. Baltimore: Johns Hopkins University Press, 1997.

Arms, William Y. 'Quality Control in Scholarly Publishing on the Web: What Are the Aternatives to Peer Review?', in: Journal of Electronic Publishing 8, 2002. Available at: <http://www.press.umich.edu/jep/08-01/arms.html>

Birkerts, S. The Gutenberg Elegies: The Fate of Reading in an Electronic Age. Boston: Faber \& Faber, 1994.

Day, Michael. 'E-print Services and Long-term Access to the Record of Scholarly and Scientific Research', in: Ariadne 28 (June 2001). Available at: <http://www.ariadne. ac.uk/issue28/metadata/>

Day, Ronald E. The Modern Invention of Information: Discourse, History, and Power. Carbondale: Southern Illinois University Press, 2001.

De Roure, David, Nicholas R. Jennings, and Nigel R. Shadbolt. The Semantic Grid: A Future E-Science Infrastructure. 2003. Available at: <http://www.semanticgrid.org/ documents/semgrid-journal/semgrid-journal.pdf>

Duff, A.S. 'Daniel Bell's Theory of the Information Society', in: Journal of Information Science 24, 373-393, 1998.

Febvre, Lucien and Henri-Jean Martin. L'apparition du livre. Paris: Michel, 1958.

Ferris, Sharmila Pixy. 'Writing Electronically: The Effects of Computers on Traditional Writing', in: Journal of Electronic Publishing 8, 2002. Available at: <http://www.press. umich.edu/jep/08-01/ferris.html8>

Gross, Alan G., Joseph E. Harmon, and Michael Reidy. Communicating Science: The Scientific Article from the 17th century to the Present. Oxford: Oxford University Press, 2002. 
Guntjahr P.C. and M.L. Benton, Illuminating Letters: Typography and Literary Interpretation. Amhurst: University of Massachusetts Press, 2001.

Harnad, Steven. 'Implementing peer review on the net: scientific quality control in scholarly electronic journals'. 1996. 103-108. Available at: <http://cogprints.ecs.soton.ac.uk/ archive/00001692/00/harnad96.peer.review.html>

Harnad, Steven. 'The subversive proposal'. 1995. Available at: <http://www.arl.org/scomm/ subversive/sub01.html>

Kirschenbaum, Matthew. 'Materiality and Matter and Stuff: What Electronic Texts are Made Of', in: Riposte, 12, 2001. Available at: <http://www.altx.com/ebr/riposte/rip12/ rip12kir.htm>.

Kling, R. and G. McGim. 'Scholarly Communication and the Continuum of Electronic Publishing', in: Journal of the American Society for Information Science 50, 890-906, 1999.

Levy, David M. 'The Universe is Expanding: Reflections on the Social (and Cosmic) Significance of Documents in a Digital Age', in: Bulletin of the American Society for Information Science, 25, 4, 17-20, 1999.

Mackenzie Owen, J.S. and J. van Halm. Innovation in the Information Chain: The Effects of Technological Development on the Provision of Scientific and Technical Information. London and New York: Routledge, 1989.

Mackenzie Owen, J.S. Scientific Communication and the Electronic Journal. Forthcoming.

McGann, Jerome. The Textual Condition. Princeton: Princeton University Press, 1991.

Nentwich, Michael. 'Quality Filters in Electronic Publishing' in: Journal of Electronic Publishing 5, 1999. Available at: <http://www.press.umich.edu/jep/05-01/nentwich. html>.

Olson, D.R. The World on Paper: The Conceptual and Cognitive Implications of Writing and Reading. Cambridge University Press, 1994.

Ong, Walter J. Orality and Literacy: The Technologizing of the Word. London: Methuen, 1982.

Popper, Karl R. Objective Knowledge: An Evolutionary Approach. Oxford: Clarendon Press, 1972.

Reddy, Michael J. 'The Conduit Metaphor: A Case of Frame Conflict in our Language About Language', in: Andrew Ortony (ed.), Metaphor and Thought, 284-297. Cambridge: Cambridge University Press, 1993.

Roberts, R.J. 'Scholarly Publishing, Peer Review and the Internet', in: First Monday 4, 1999. Available at: <http://www.firstmonday.dk/issues/issue4_4/proberts/index.html>.

Rockwell, Geoffrey and Lynne Siemens. The Credibility of Electronic Publishing: Report on Responses to the Questionnaire. 2000. Available at: <http://web.mala.bc.ca/hssfc/ Final/QuestionnaireR.htm>.

Rowland, F. 'Print Journals: Fit for the Future'. Ariadne. January 1997. Available at: <http://www.ukoln.ac.uk/ariadne/issue7/fytton/>.

Rusch-Feja, Diann. 'The Open Archives Initiative and the oaI Protocol for Metadata Harvesting: Rapidly Forming a New Tier in the Scholarly Communication Infrastructure', in: Learned Publishing, 15 (July 2002): 179-186.

Svedjedal, Johan. The Literary Web: Literature and Publishing in the Age of Digital Production - a Study in the Sociology of Literature. Acta Bibliothecæ Regiæ Stockholmiensis. Stockholm: Kungl. Biblioteket, 2000.

Thiel, Sh. 'The Online Newspaper: A Postmodern Medium', in: Journal of Electronic Publishing 4, 1998. Available at: <http://www.press.umich.edu/jep/04-01/thiel.html>.

Thompson, John B. The Media and Modernity: A Social Theory of the Media. Cambridge: Polity Press, 1995.

Wagner, T. and S. Graham. Efficient Self-Versioning Documents. 1997. Available at: $<$ http://citeseer.nj.nec.com/wagner97efficient.html>.

Weaver, W. 'Recent contributions to the mathematical theory of communication', in:

Shannon, C.E., and Weaver, W. The mathematical theory of communication. University of Illinois Press, Chapters 1.2, 4-6, 1949.

Weller, A.C. 'Editorial Peer Review for Electronic Journals: Current Issues and Emerging Models', in: Journal of the American Society for Information Science. 51, 1328-1333, 2000. 


\section{Authenticity in}

\section{Bis and By}
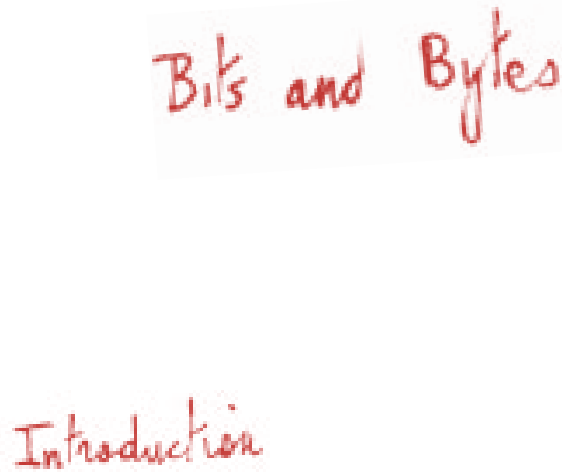

Writing has been instrumental in bringing mankind to where it is today, without it, accumulating the amount of knowledge currently at our disposal would have been unthinkable. Writing has also shaped the legal system into what we know today. The existence of nation-states with national laws, as opposed to local customary law, is to a large extent a legacy of the printing press, which allowed for legal texts to be spread fairly quickly over large regions. ${ }^{I}$ In this legal landscape, handwriting has continuously served as the authentication method of choice.

The development of information technology is a revolution of at least the same magnitude as the printing press. We are only beginning to see its implications for society in general and the law in particular. In this paper, a very small aspect of this revolution will be explored, namely the issues involved in finding a substitute for the handwritten signature suitable for the information society. To narrow the subject down further, the focus will be placed solely on developments in western continental Europe, with Belgian law as a case study.

In the first section, a brief overview will be presented regarding the role played by handwriting in general and the signature in particular throughout history. Subsequently, two practical cases - the private contract and the last will and testament - will be discussed in order to demonstrate the role that handwriting still plays in the current legal system. In the following section, the transition to digital writing will be discussed, starting with an introduction to digital signature technology and continuing with a short exposition on the legislation enacted to pave the way for electronic signatures in the European Union. Finally, a comparison will be made between handwritten and digital signatures as legal authentication methods.

In this paper, I will argue that, although legislation was enacted to accommodate electronic signatures, ample evidence remains that the paradigm of handwriting has not yet been traded for a new one. From a short-term perspective, this does not appear to be problematic. However, in the long term a number of serious issues surface with regard to the durability of digital documents. 


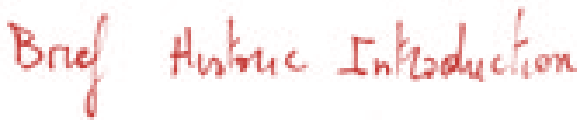

Before diving into the turbulent waters of the digital age, a brief excursion into legal history is in order. Writing has not always enjoyed the privileged status that it currently has in the eyes of the law; in Roman and medieval times, for example, witness testimony was generally preferred, as it was possible to engage in cross-examination. From the 16th century onwards, documentary evidence gradually gained increasing legal standing. In western continental Europe, this evolution culminated in the evidence rules laid down by the Napoleonic codes, which firmly established the prevalence of documents over witness testimony (Macneil 2000, 7-9; Van Eecke 2004, 77). Documentary evidence became mandatory for certain transactions and was the only form of evidence admissible in court, with the express exclusion of witness testimony. ${ }^{2}$

The use of handwritten signatures as a method of authentication is also a relatively recent custom. Any use of documentary evidence - as such or in support of witness testimony - entailed the inclusion of a reference to the person bound by the document in question. Throughout history, seals, signets and handwritten marks were used for this purpose. In Roman and medieval times, seals and signets received more legal credit than handwritten marks (Van Eecke 2004, 86). As literacy increased towards the end of the Middle Ages, documentary evidence was more often drawn up by the parties themselves instead of dictated to a scribe. Gradually, the handwritten subscription which usually accompanied a seal or signet gained more legal weight as, unlike a seal or signet, only the signatory was capable of placing it.

This evolution was compounded by the introduction of surnames at the end of the 10th century. At the outset, the choice of a surname was more or less free, but by the 16th century it was customary to pass on a father's surname to all of his children (Van Eecke 2004, 104; Pintens 1981, 14). From that time, the handwritten signature, much as we know it today, all but replaced the use of seals and signets in western continental Europe (Van Quickenborne 1985; Van Eecke 2004, 87).

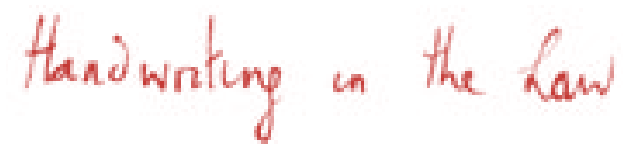

Two examples demonstrate the role that handwriting still plays in the current legal system: namely the private contract and the last will and testament. In terms of the Napoleonic tradition, a binding agreement is concluded when the parties agree on its contents (Article 1108, Belgian Civil Code). An oral agreement as such is valid and binding. However, a document signed by all parties involved must be drawn up for agreements exceeding a specified value. This document is sufficient evidence of the agreement and contradicting witness testimony is inadmissible in court (Article 1341, Belgian Civil Code). The reasons for this preference are pragmatic: the terms of an oral agreement are notoriously difficult to prove in court. The law goes one step further for some exceptional transactions by demanding that a document be drawn up as a requirement for the legal act to exist, in addition to proving its 
terms. It is often the case that certain predefined handwritten notifications must be included in the document as well. ${ }^{3}$

Another legal act that calls for handwriting is the last will and testament. In Belgium, as in many other countries, a will can take the form of either a public instrument; a holographic will; or a so-called international will. In the first two cases, handwriting is an essential requirement for the validity of the will. To this date, a public will requires that the testator dictate his dispositions to the notary in the presence of two witnesses, or alternatively, two notaries (see Articles 971-975 of the Belgian Civil Code). The notary is obliged to record the will in person and by hand. Afterwards, the will must be read aloud before it is signed by all present. The presence of witnesses, by and of itself, is still sufficient evidence of the contents of the will. In the opinion of the Court of Cassation, it is irrelevant whether the witnesses are paying attention to what is being said, as long as they are in a position to verify whether all formal requirements were fulfilled. 4

As the name implies, the holographic will is a document entirely handwritten by the testator (see Article 970 of the Belgian Civil Code). To be valid, it must be dated, signed, and it must mention where it was drawn up. Witnesses are not required and the will does not have to be registered to be valid.

The international will, by contrast, does not have to be written by hand. 5 The will must be handed over to a notary in the presence of two witnesses, either openly or in sealed form. The testator must declare the piece to be his last will and must then sign it or formally recognize his signature if it is already present. Then, the notary must date and sign the will. Finally, the witnesses must place their signature at the bottom of the will. The notary must fill out and sign a form in duplicate, stating that all of the formalities have been fulfilled. The will is sealed in the presence of the testator and both witnesses and is then archived by the notary.

From these examples, it is apparent that the signature is the prima donna of evidence law. Until very recently, this term referred exclusively to handwritten signatures. A manually signed document is traditionally granted such high probative value in light of the properties that legal scholars attribute to it (see Dumortier, Van Eecke, and Anné 1999, 54-56; Gobert, and Etienne Montero 2004, 220-230). Firstly, the signature identifies its author in a unique way. Secondly, the signature expresses consent with the contents of the document. For this reason it has always been insisted upon that the signature is placed by hand directly upon the document itself, the use of carbon paper, seals or stamps is prohibited. ${ }^{6}$ According to some scholars, the signature protects the integrity of the document as it signals that the document is complete (see Dumortier, Van Eecke and Anné 1999, 52; Van Eecke 2004, 152; Van Quickenborne 1985, 5-6). Any additions below the signature or in the margins of the text are to be disregarded unless they, themselves, are signed. Each page of a long document is often signed separately to ensure pages are not inserted or replaced afterwards.

The high regard in which a signed document is held, is most obvious in the difference in status between an original and a copy. Only an original document is sufficient documentary evidence of agreements exceeding a certain value (see Article 1341 of the Belgian Civil Code; Verheyden-Jeanmart 1991, $201 \mathrm{ff}$ ). The essential 
characteristic of an original document is precisely that the signature was placed directly upon it; any document derived from the original, but lacking an original signature, is merely a copy with less probative value. The method used to create the copy, for instance photocopying; use of carbon paper; or scanning is of no importance. The copy is admissible in court, but the adversary may demand the production of the original. However, if the copy is not challenged, the judge must presume it to be a faithful rendering of the original and treat it as such.

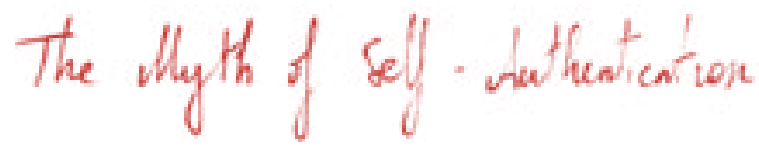

In the hierarchy of evidence, the signed document is the highest form of proof. In view of the properties attributed to it in legal literature, the original signed document appears to be a self-authenticating form of proof. Upon closer examination, this picture becomes somewhat unraveled (Dumortier, Van Eecke, and Anné 1999, 54).

Where the identification function is concerned, handwriting in general, or a signature in particular, only serves as a reliable way to identify the author under the right conditions. When a signed document is presented as evidence in court, the presumed author is usually already known. Without any contextual data, identifying the author of a particular piece of handwriting is like searching for a needle in a haystack. In principle, the signature should consist of the signatory's last name, but this has been interpreted with some flexibility by the courts; the Court of Cassation emphasizes that the signature must be the handwritten mark that the signatory usually places in order to manifest himself towards others. ${ }^{7}$ In this spirit, even signatures that are illegible to the point that they give no clue whatsoever about the signatory's identity are often accepted. The bottom line is that verification by the court in the case at hand must be possible (see Van Quickenborne 1985, 22). Clearly, far from providing instant identification of its author, a signature generally requires the presence of contextual information to perform this function. The necessary context can consist of an acknowledgement by the signatory or the availability of reference signatures.

Even when it is known who the supposed author is, there is still the issue of forgery. So real is the risk of forgery that the law allows the presumed signatory of a contract to denounce 'his' signature as fake, and his heirs may suffice by saying they are not familiar with the testator's handwriting or signature - in either case, an expert witness must be appointed to determine whether the signature is genuine or not (Articles 1323-1324, Belgian Civil Code).

Concerning the second function, inducing consent merely from the presence of a signature is not self-evident either. Clearly, it is only by legal convention that the handwritten signature implies consent with the contents of a document, be it a contract or a will. Moreover, signatures are placed in other circumstances without implying consent, for instance, the witnesses present at the drafting of a public will do not express consent by signing, but only signal their presence at the occasion. 
With regard to the third function - guaranteeing the integrity of documents - signatures are not the most efficient method to use. Primarily, it is not the signature, but the medium of paper, which guarantees the integrity of a document (see Gobert and Montero 2000, 23; Wilms 1995-1996, 839). Although not impossible, it is difficult to alter text on a page in an undetectable way. Documents that are entirely written by hand offer somewhat more protection against alterations by third parties, although they remain vulnerable to alterations made by the original author. The law takes these risks into account by making forgery a criminal offence. Also, to limit the risks in a contractual setting, the parties are required to create as many originals of bilateral agreements as there are parties with a distinct interest. Some very important transactions must be entrusted to a notary, who is responsible for guarding the integrity of his archives.

Even though in reality the handwritten signature does not live up to all its ascribed functions perfectly, these functions were still normative in the search for a substitute suitable for the information society.

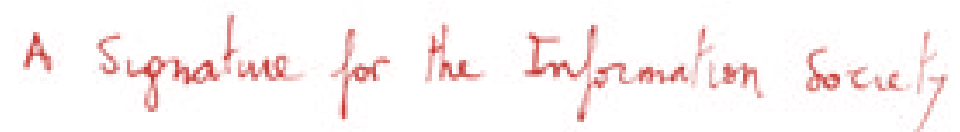

Paper is no longer the medium of choice to record, distribute, and receive information, as, to a large extent, information and communication technology (ICT) has taken its place. Evidently, as this technology is based on electronic pulses represented by zeros and ones, ICT does not accommodate handwriting very well. For legal systems that only recognize manual signatures, the writing was on the wall - an alternative had to be found to fulfill the functions of the signature, and, by extension, of handwriting.

A guiding principle in the adaptation of the law to the digital age is functional equivalence theory, a model first used by the UN Commission on International Trade Law (UNCITRAL) for the development of the Model Law on Electronic Commerce in 1996. This approach starts from an analysis of the purposes and functions underlying traditional paper-based requirements with a view to determining how those purposes or functions could be fulfilled electronically (see $<$ http://www. uncitral.org/english/texts/electcom/ml-ecomm.htm>).

Bearing functional equivalence in mind, the legal world saw great potential in asymmetrical encryption technology as the ideal electronic substitute for the handwritten signature. This technique was promptly dubbed digital signature technology. Asymmetrical encryption certainly presents some useful properties for authentication purposes, although it is not without its limitations.

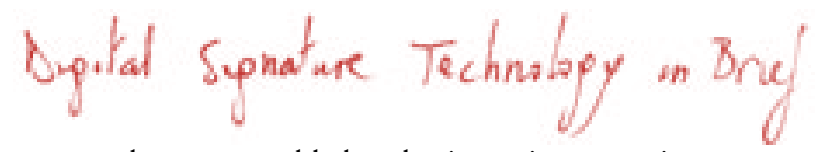

Digital signatures do not resemble handwritten signatures in any way. Where the handwritten signature is a graphical mark with a more or less stable form, the digital signature is unique for each file to which it is appended. The reason for this is 
that the digital signature is derived from the file to which it belongs by means of a series of complicated mathematical computations.

In a first step, a hash value or so-called digital fingerprint is calculated for the file to be signed. Hashing is a technique by which electronic information can be reduced to a unique fixed-length code: if even a single character in the file is modified in transmission or storage, the resulting hash value will change. By comparing the original hash value with the current one, one can determine whether a document has been altered or not. Of course, the original digital fingerprint must be safeguarded against manipulation if it is to be compared later on with a newly calculated hash value. This is where encryption comes in. Encryption entails that a plain text message is transformed into a cipher text that seems meaningless. Symmetrical encryption means that the encryption key is only shared by the sender and receiver of the message. This protects against manipulation by third parties, but both sender and receiver could impersonate the other by using the common key. Asymmetrical encryption avoids this problem by giving each party his own pair of keys, one that must remain secret and another that may be made public. A text encrypted with one of the keys can only be decrypted with the corresponding key from the pair.

Asymmetrical encryption can serve two purposes. Alice can use Bob's public key to encrypt a message and send it on to him and, as only Bob has the corresponding private key at his disposal, no one else will be able to decrypt the message. Confidential messages can be sent safely through open networks this way. Conversely, Alice can encrypt a message with her private key and send it to Bob, who uses Alice's public key to decrypt it. As Alice's key is publicly available, anyone can decrypt this message. The point here is that Bob can be sure that Alice is the author of the message, as only she knows the corresponding private key.

There is one caveat to this story, however. Neither the hash value, nor the private or public key refers to Alice's identity in any way, as these are just numerical values. In this sense, the term digital seal would be more accurate for this technology. Bob must use other means to find out who the rightful owner is of the public key. Perhaps it was created by Carl, who is impersonating Alice. Alice and Bob could exchange public keys in a face-to-face meeting, but this is not always feasible. Also, Carl may have stolen the private key from Alice after this meeting took place. In open network environments, like the Internet, a public key infrastructure must be in place in order to tie public keys to the identity of their rightful owners.

A public key infrastructure offers a number of services related to digital signatures. Firstly the service of creating key pairs with which digital signatures can be placed. A second service is public key certification, whereby the link between a public key and its rightful owner is published for the benefit of recipients of signed documents. Depending on the type of certificate, the owner's identity is checked more or less thoroughly. Typically, a directory is kept of all certificates issued as well as a revocation list, which is important in the case of the theft of a private key. Lastly, time-stamping is an important service, as it is necessary to know whether a digital signature was placed before or after revocation of the public key certificate. To summarize, a digital signature is a small, encrypted file that is derived from the 
file to be authenticated. To validate the signature, the signatory's public key must be used to decrypt the signature and information about the public key's rightful owner must be obtained. ${ }^{8}$

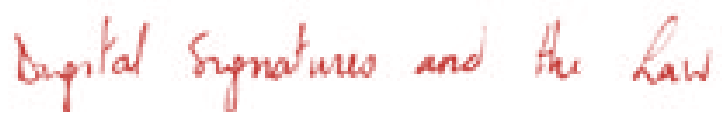

The properties of digital signatures as described above attracted great interest from the legal community around the world. A technology that allows one to identify the sender of a message, guarantee its integrity, and preclude repudiation by the sender after the fact is a prime substitute for handwritten signatures in the information society. In Europe, several legislative initiatives emerged to codify digital signatures. ${ }^{9}$ In order to ensure a harmonized legal framework for the internal market, the European Union issued Directive 1999/93/EC on electronic signatures. ${ }^{\text {IO }}$

Initially, the idea was to enact rules attaching legal value to digital signature technology but this path was subsequently abandoned due to concerns about the longevity of a law tied to one type of technology. Therefore the legislators carefully avoided explicit references to digital signature technology in the wording of the directive. The term electronic signature is used and given a broad meaning in an attempt to create a technologically neutral legal framework.

An electronic signature is any data in electronic form that is attached to or logically associated with other electronic data and serves as a method of authentication (Article 2, $1^{\circ}$ E-signature Directive). A regular e-mail with the sender's name placed at the bottom can be considered an electronically signed document in this sense. The legal value attached to electronic signatures as such is fairly weak. Electronic signatures may not be discriminated against in court just because they are electronic (Article 5, $\$ 2$ E-signature Directive), but they may be dismissed on other grounds.

A stricter definition is given for a subcategory of electronic signatures, namely the advanced electronic signature. The requirements are that the signature is uniquely linked to the signatory and is capable of identifying the signatory, that it is created using means under the sole control of the signatory and that it is linked to the relating data in such a manner that any subsequent change of the data is detectable (Article 2, $2^{\circ}$ E-signature Directive). In the current state of technology, only digital signature technology can fulfill all these requirements.

Advanced electronic signatures, accompanied by a qualified certificate and created by a secure-signature-creation device, enjoy a special status in the directive. In legal literature this type of advanced electronic signature is usually termed a qualified electronic signature. Such qualified electronic signatures must be admissible as evidence in legal proceedings and must receive the same legal consequences as a handwritten signature would in similar circumstances (Article 5, $\$ 1$ E-signature Directive). The benefit of the qualified electronic signature is that it has the same legal value in the whole internal market.

In transposing the E-signature Directive, the Belgian legislator literally copied the definitions of electronic signatures in general and qualified electronic signa- 
tures in particular. ${ }^{I I}$ In contrast to the directive, the advanced electronic signature was given specific legal consequences as well. ${ }^{12}$ For the purpose of creating original proof of a private agreement, the handwritten signature may be replaced by an electronic signature that can be attributed to its author and that guarantees the integrity of the document it is supposed to authenticate.

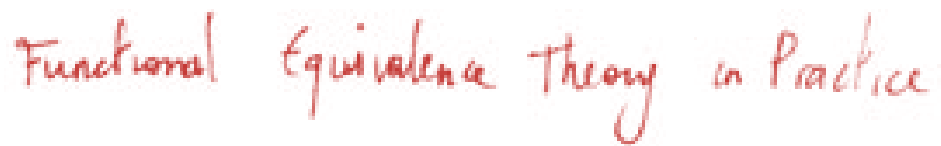

With regard to digital signature technology, two questions arise: How well do digital signatures perform the authentication functions expected of a signature and how do they compare with handwritten signatures?

Concerning the identification function, a digital signature alone does not reveal the signatory's identity, as it is just a computer code. Unlike a handwritten signature, a digital signature does not contain any direct reference to a person. However, depending on the contents and the reliability of the accompanying certificate, the signatory can be readily identified. Thus, both the handwritten and the digital signature require the presence of contextual information in order to be useful, albeit for different reasons.

The theft of a private key is problematic, as is forgery in the case of handwritten signatures. By law, the legal risks of this happening are distributed among the parties involved. The rightful owner is responsible for the use of his private key, until he revokes it (Article $19 \$ 2$ CSP Law). If the owner neglects to do so, the owner can be held liable for any damages under tort law. After revocation, any recipient of a signed document must consult the revocation list. Of course, the person unlawfully using the private key can be sued for damages by either party (Montero, 43-45).

When it comes to the expression of consent, it is but a social or legal convention that placing a signature entails consent to the signed document, as is the case for handwritten signatures. Digital signature technology makes this fact all the more evident, as machines are perfectly capable of digitally signing documents without any human intervention. Already, various automatic processes use digital signature technology for security reasons, with no intention whatsoever of expressing consent on anyone's behalf. Digital time stamps, for example, are nothing more than digitally signed text files containing the hash value of a file and an indication of the time of receipt.

With regard to the integrity function, digital signatures are touted as the perfect way of ensuring the integrity of signed documents. Although not false, this claim should not be taken at face value. Firstly, digital signature technology does not actively protect integrity, it only signals if the integrity of the bitstream has been compromised. Modifications in the bitstream may or may not entail a significant change in the contents of the signed message. If the money owed in a contract is changed from 100 to 1,000 per item, the integrity of the contractual terms is clearly compromised. If one pixel in a photograph changes from one shade of gray to another, this is probably not at all relevant for the message conveyed. Single bits 
'falling over' is a common occurrence and rejecting any file where this has occurred would be overreacting. Digital signature technology is only capable of signaling integrity on the bit level, not on document level. This limitation makes it a rather fragile authentication tool.

Digital signatures make no pretense whatsoever of producing self-authenticating documents. This technology claims to be functionally equivalent to the handwritten signature, which does appear justified, at least if one only takes short-term perspectives into account.

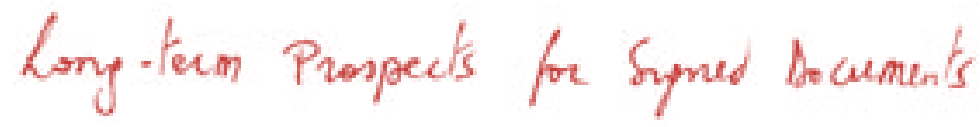

The validity of documentary evidence, notably wills and contracts, usually only becomes an issue a long time after their creation. In comparing handwritten and electronic signatures, the durability of these authentication techniques must be investigated as well.

On average, manually signed paper documents are easy to preserve, even for extended periods of time. Under the right conditions, paper records can be kept for hundreds of years. Several copies of the Gutenburg Bible, printed in 1455, still exist today (see <http://en.wikipedia.org/wiki/Gutenberg_Bible>).

By contrast, the shelf life of digitally signed documents is dubious. As hinted above, the greatest strength of digital signature technology, signaling manipulation of the bitstream, is also its greatest weakness. Validation of a digital signature is only possible as long as the original bitstream remains perfectly intact. The perfect storage of bitstreams, even for a relatively short time, remains a challenge in itself. Even storage media that are specifically designed for this purpose, like CDwORM disks, suffer from bit degradation to the extent that all readers come equipped with software to correct errors (see Boudrez, 10; Starret 2000). Digital signature technology by itself does nothing to prevent any changes to a bitstream.

Storing computer files intact is only part of the story. In itself, a bitstream is of little interest to us as it is not readable by humans. Both the necessary hardware and software must be available to translate the bitstream into an intelligible format on screen or in print. Hardware and software platforms come and go at an alarmingly high rate, resulting in the obsolescence of the file formats that depend on them. An example of what this can lead to is the case of the CNEs (Centre National des Etudes Spatiales, France), which was forced to have documents that were created in 1985, re-typed manually in 1990 and again in 1997, because the newer generations of word processors could not read the original files with sufficient accuracy (Valoris 2003, 38). In order to cope with file format obsolescence, archivists generally put forward two distinct strategies, namely migration and emulation.

Migration entails the translation of a computer file into a suitable archival format. Depending on the specifics of the format chosen, the characteristics of the original file may be preserved or lost. For instance, when converting Ms Word files to flat file, the original look and feel is lost; if the same ms Word files are migrated 
to uncompressed Tiff files, the original look and feel of the document is preserved, but the ability to reuse the content is lost instead. Distinguishing between essential and incidental characteristics is the responsibility of the archivist. Whichever format is chosen, migration breaks any digital signatures accompanying the original file, as the new file is represented by its own distinct bitstream.

With regard to the preservation of digital signatures, emulation appears to be a more promising archival strategy. The functions and behavior of the obsolete platform - meaning the old hardware, software or both - is recreated on a contemporary computer platform, allowing the original files to be accessed. Notwithstanding some exceptions, current emulators are still in a highly experimental phase of development. ${ }^{\text {I3 }}$ Developing emulators is very difficult, especially for platforms that are not fully documented, and as a consequence, the costs involved are considerable. It is highly unlikely that emulators will be created for all possible platforms that exist today and for all future platforms to come. Moreover, emulators developed to run on today's platform will become obsolete in their turn, making either a chain of emulators or constant redevelopment necessary. If this chain is broken or redevelopment is omitted, the original file is lost, as it can no longer be accessed (Boudrez 2005, 83-84). The fact that the digital signature accompanying the file can still be validated offers little consolation.

Even if abstraction is made of these hurdles, the digital signature itself is at risk of becoming obsolete. Asymmetrical encryption works on the assumption that it is practically infeasible to crack the code by trying all the possible key combinations because the necessary computer power is not available. As time goes by, however, ever more powerful computers are developed, and eventually trying all the key combinations becomes a distinct possibility. Alternatively, flaws may be found in the encryption or hash algorithm, opening up new avenues of attack (Libon and Van den Eynde 2000, 22-23). To alleviate these problems, the length of the encryption keys is increased to match the pace of computer development and new encryption algorithms are introduced. Of course, this does not offer a solution for the legacy of digital signatures. Once the key or the algorithm is broken, fake digital signatures, indistinguishable from genuine ones, can be created.

One proposed solution to the weakening of digital signatures, is re-signing old signatures with more recent technology (see Blanchette 2004, 24 ff.; Libon, and Van den Eynde 2000,17-32). Although certainly technically feasible, this scenario quickly becomes very cumbersome. Not only the obsolescence of the digital signature itself must be taken into account, but also of the certificate identifying its rightful owner and any available time stamps. Such certificates and time stamps are no more than small files digitally signed by the service provider.

Archivists feel that the investment in time and effort to preserve fully functional digital signatures is just not worth it. Instead of applying costly procedures to ensure the integrity of the signature and the entire validation chain behind it, they propose to invest only in ensuring the integrity of the document itself. In this scenario, any signatures accompanying a document will be transformed into metadata, which mention who authenticated the document at a certain point in time (Blanchette 2004, 32-36 ff.). 
From a long-term perspective, digital signatures perform poorly in comparison with handwritten signatures. This does not imply that digital signature technology should be abandoned, only that appropriate measures should be taken to counter such shortcomings. Several technical and organizational possibilities are proposed by archivists and engineers, but the question remains to what extent these are accommodated by the legal framework.

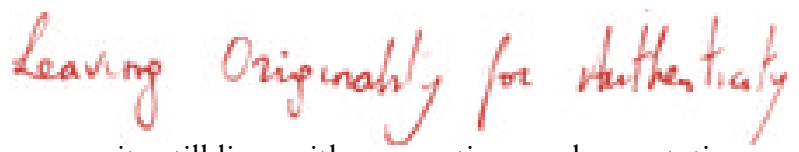

The legal community still lives with assumptions and expectations rooted in the paper environment. One of these is the idea that the original signature must be preserved. For paper documents, this is a reasonable requirement, as a handwritten signature on a paper medium is relatively easy to preserve, even for extended periods of time. Moreover, the signature continues to fulfill its authenticating functions more or less identically throughout its life span. Thus an original document provides the same clues to determine its authenticity on the day of its creation as it does 50 years later.

Originality is a good proxy for authenticity with regards to paper documents, but this is not the case for digital records. As digital signatures are difficult to preserve and lose their authenticating functions after a relatively short period of time, indications about the authenticity of digital records should be sought elsewhere. The most obvious source of clues about document authenticity is the system in which these documents reside. Of course, the reliability of a record-keeping system is a function of the trustworthiness of its keeper.

By way of example, the e-invoicing Directive takes a clear step away from requiring originality in favor of a more direct evaluation of authenticity. ${ }^{I 4}$ Electronic invoices are valid as long as the 'authenticity of their origin and the integrity of their content' remain guaranteed. However, member states may impose invoices to be preserved in the form they were sent, either on paper or electronically and thus may choose to uphold the originality requirement to some extent, but they are not encouraged to do so.

In the e-signature Directive, the originality requirement is still present in both concepts of advanced and qualified electronic signatures. By definition their creation must be under the sole control of the signatory and the signatures themselves must guarantee the integrity of the document they accompany (see Art. $2 \$ 2 \mathrm{~d}$ Electronic Signature Directive). Qualified electronic signatures enjoy a privileged legal status, but their preservation along with the entire validation chain is cumbersome.

A very tentative step away from the originality requirement can be found in the generic definition of electronic signatures as given in the E-signature Directive. Any electronic data which serves as a method of authentication falls within its scope. Hence not only an original electronic signature may qualify, but also reliable metadata detailing validation results of defunct advanced or qualified signatures. However, the legal status of generic electronic signatures is somewhat vague 
and undefined. The member states need only ensure their admissibility before the courts on a non-discriminatory basis; this means that an electronic signature may not be rejected just because it is electronic, though it may be denied legal value because the technology used is unreliable. Still, this concept opens the door for the courts to evaluate the authenticity of the documents presented to them directly by assessing the reliability of record-keeping systems and the trustworthiness of their keepers.

In Belgian law, the admissibility of generic electronic signatures has been introduced as mandated, though the advanced electronic signature has taken precedence in the areas of law where it matters most, for instance, regarding the law on evidence of contractual obligations. The revised Civil Code starts off by broadly stating that an electronic signature must be attributable to its author, but then it goes on to say that the signature itself must guarantee document integrity. In other words, the originality requirement still shines through. There are some preexisting exceptions to the originality requirement that may provide a backdoor for a more direct approach to examining document authenticity. However, this only provides a solution for a limited number of cases.

As it stands, the law favors emulation as an archiving strategy. In practice, there are other factors to be taken into account, specifically cost-effectiveness and risk. In the end, it comes down to the parties involved to decide what costs they are willing to incur in order to preserve their digitally signed documents. Some will opt to preserve their digital signatures complete with validation chain, in order to benefit from their privileged legal status. Others may decide to entrust their signed records to a third party custodian, vouching for authenticity on the basis of the reliability of his record-keeping system. As standard best practices with regard to the preservation of digitally signed documents evolve, the inappropriateness of the originality requirement for digital documents will become increasingly apparent.

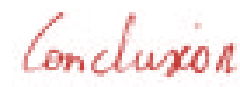

The rise of the information society has forced legal scholars to look at basic concepts from an entirely different angle. One result of this exercise has been the enactment of legislation paving the way for electronic signatures. Upon closer inspection, ample evidence exists that legislators have yet to trade the paradigm of handwriting for a new one. The legal value of the qualified electronic signature is explicitly linked to that of handwritten signatures, which marks it as a transitional concept. The definition of the advanced electronic signature mimics the workings of handwritten signatures. As a consequence, this type of signature is rigidly bound to its original form, making signed documents highly vulnerable to technological obsolescence. The generic electronic signature does not suffer from these constraints, but the legal certainty it provides is low. Basically, citizens can either strive to fulfill the originality requirement for their digitally signed documents or venture into uncharted territory. 
Although electronic signatures are called upon in the legal world to play the same role as handwritten signatures, both authentication methods function according to a different logic. Looking at the long-term perspective for signed documents makes this very clear. Instead of trying to force one into the mould of the other, these differences should be acknowledged in an appropriate way by the legal system. For one, the appropriateness of the originality requirement should be assessed in this light. More generally, a legal frame of reference should be developed for the evaluation of authenticity and authentication of digital documents.

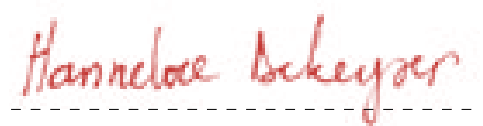

SIGN HERE! 
Notes

1. The influence of the media used for communication on law is the subject of Katsch, Ethan, The Electronic Media and the Transformation of Law, 347. New York: Oxford University Press, 1989.

2. Articles 1320 and 1341 of the French Civil Code of March 21, 1804. Subject to various modifications, this Civil Code is still in force in France and Belgium.

3. Two examples are the unilateral promissory note pertaining to a sum of money (Art. 1325, Belgian Civil Code) and the consumer credit agreement (Art. 14 of the law of June 12, 1991 on Consumer Credit, M.B, July 9, 1991), which must both contain certain handwritten notifications in order to be valid.

4. Belgian Court of Cassation, May 4, 1979, Pas., 1979, I, p. 1047-1048, excerpt available on <http://www.cass.be $>$.

5. The convention providing a Uniform Law on the Form of an International Will (Washington, DC, October 26, 1973) was introduced into Belgian law by the law of January 11, 1983 (Moniteur Belge, October 11, 1983).

6. See Belgian Court of Cassation, June 28, 1982, Pas., 1982, I , p.1286-1292. and Van Quickenborne, M., 1985, p. $23 \mathrm{ff}$.

7. A holographic will inscribed simply with 'mother' was denied any legal value, although there was no discussion about the testator's identity, see Belgian Court of Cassation. January 7, 1955, Pas., 1955, I, p. 456 and Van Quickenborne, M., 'Quelques réflexions sur la signature des actes sous'. In Révue C.J.B., 10, 1985.

8. For a more detailed description of digital signature technology, see Dumortier, J., P. Van Eecke, and I. Anné, The Legal Aspects of Digital Signatures, II, I.c., Gent: Mys and Breesch 1999, 21-48.
9. The German Bundestag passed the Multimedia Law (Gesetz zur Regelung der Rahmensbedingungen für Informationsund Kommunikationsdienste, July 22, 1997, Bundesgesetzblatt, I, 1997, p. 1870) and Italy passed law no. 59 of March 15, 1997 to allow the use of electronic documents for legal transactions. Around the same period, other European nations were preparing similar legislation, see Dumortier, J., P. Van Eecke, and I. Anné, The Legal Aspects of Digital Signatures, IV, Gent: Mys and Breesch 1999, 125.

10. See Directive 1999/93/EC of the European Parliament and of the Council of December 13, 1999 on a Community framework for electronic signatures, Official Journal, January 19, 2000, L13/12 (hereafter E-signature Directive), available on $<$ http://www.europa.eu.int/eur-lex/nl/index.html $>$.

11. See the law of July 9, 2001 on a legal framework for electronic signatures and certification services, hereafter CSP Law (M.B. September 29, 2001).

12. See Art. 1323 as amended by the Law of October 20, 2000, on the use of communication technology and the electronic signature in judicial and non-judicial proceedings (M.B. December 12, 2000).

13. Emulators have been successfully developed for various game consoles, but also for other computer systems, see $<$ http://en.wikipedia.org/wiki/List_of_emulators $>$.

14. Directive $2001 / 115$ amended Directive $77 / 388 / E E C$ with a view to simplifying, modernizing and harmonizing the conditions laid down for invoicing with respect to value-added tax, O.J., L 15/24.

\section{Works Cited}

Belgian Civil Code, Articles 970, 971-975, 1108, 1341 and 1325.

Belgian Court of Cassation, January 7, 1955, Pas. 1955, I, p. 456; May 4, 1979, Pas., 1979, I, pp. 1047-1048, excerpt available at: <http://www.cass.be>; and June 28, 1982, Pas., 1982, I , 1286-1292.

Blanchette, J.-.F. La conservation de la signature électronique: perspectives archivistiques.

Paris: La Documentation Française, 2004.

Boudrez, F. 'Electronic Record Keeping', in: Boudrez F., H. Dekeyser, and J. Dumortier, Digital Archiving: The New Challenge? Louvain-la-Neuve: I.R.I.S., 2005. 
Boudrez, Filip. CD's voor het Archief. Stadsarchief Antwerpen, Antwerp and Leuven: ICRI K.U. Leuven, p. 10, available at: <http:/www.antwerpen.be/david>. Directive 1999/93/ EC of the European Parliament and of the Council of December 13, 1999, Official Journal, January $19,2000, \mathrm{~L} 13 / 12$, Articles $2,1^{\circ} ; 2,2^{\circ} ; 2 \$ 2 \mathrm{~d} ; 5, \mathbb{1} 1$ and $5, \mathbb{} 2$, available at:<http://www.europa.eu.int/eur-lex/nl/index.html $>$.

Dumortier, J., P. Van Eecke, P., and I. Anné. The Legal Aspects of Digital Signatures. II, Gent: Mys and Breesch, 54-56, 1999, available at: <http://www.law.kuleuven.ac.be/ icri/>.

French Civil Code, March 21, 1804, Articles 1320 and 1341.

Gobert, Didier and Etienne Montero. 'La signature dans les contrats et les paiements électroniques: l'approche fonctionnelle', in DA/OR 2000. <http://en.wikipedia.org/wiki/ Gutenberg_Bible>. <http://en.wikipedia.org/wiki/List_of_emulators>. <http://www. uncitral.org/english/texts/electcom/ml-ecomm.htm>.

Katsch, Ethan. The Electronic Media and the Transformation of Law. New York: Oxford University Press, 1989.

Libon, O., and S. van den Eynde. European Electronic Signature Standardization Initiative - Trusted Archival Services. European Commission 2000, available on <http://www.law. kuleuven.ac.be/icri>.

Macneil, Heather. Trusting Records Legal, Historical, and Diplomatic Perspectives. Dordrecht: Kluwer Academic Publishers, 2000.

Montero, Etienne. 'Définition et effets juridiques de la signature électronique en droit belge: appréciation critique', in: Montero, Etienne (ed.), La preuve. Formation permanente, 54, Liège: ULg. Formation Permanente cup.

Multimedia Law (Gesetz zur Regelung der Rahmenbedingungen für Informations- und Kommunikationsdienste), German Bundestag, July 22nd, 1997, Bundesgesetzblatt, I, 1997.

Pintens, Walter. Naam. In: APR, Story-Scientia, Gent, 1981.

Starret, Bob. 'Compact Disc Errors', in: Roxio CD-R Newsletter, April 21, 2000, available at: $<$ http://roxio.com/en/support/cdr/cderrors.html>.

Uniform Law on the Form of an International Will (Washington, DC, October 26, 1973), Intro. to Belgian law by the law of January 11, 1983, Moniteur Belge, October 11, 1983.

Valoris, Comparative Assessment of Open Document Formats Market Overview. Report for the European Commission, DG Enterprise, 2003.

Van Eecke, Patrick. De handtekening in het recht, Van pennentrek tot elektronische handtekening. Brussel: Larcier, 2004.

Van Quickenborne, M. 'Quelques réflexions sur la signature des actes sous', in: Révue CJ B. 1985.

Verheyden-Jeanmart, Nicole. Droit de la preuve. Brussel: Larcier 1991.

Wilms, Wilfried. 'Van handtekening naar elektronische notaris - de validering van elektronische communicatie', in: R.W., 1995-1996. 




\section{Section Two}
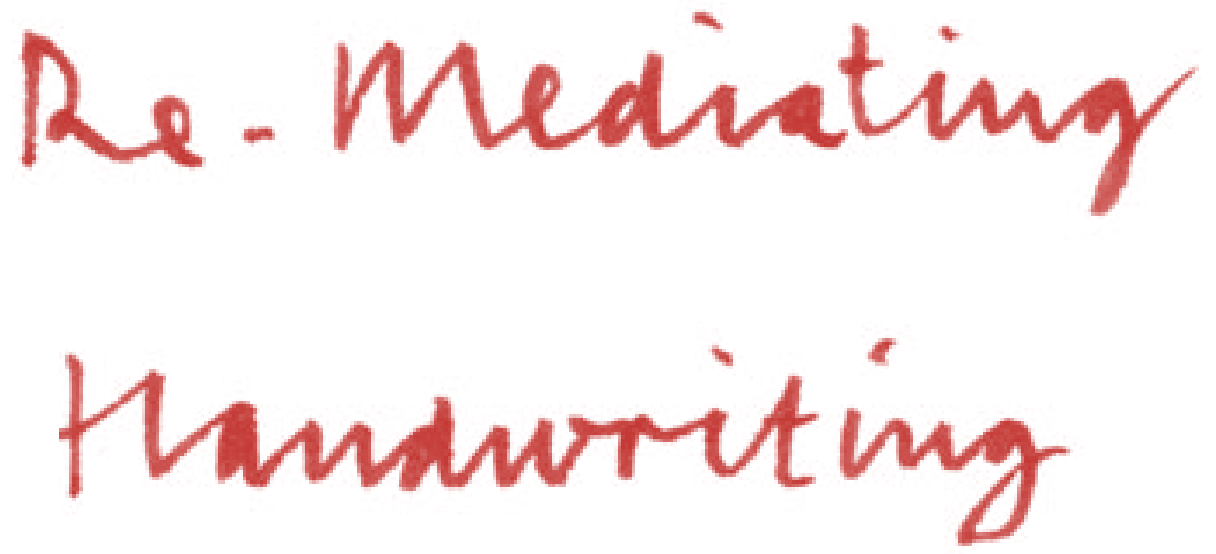



\section{Signature Jdentity Content: Handwriting in an Age of Digital Remediation}

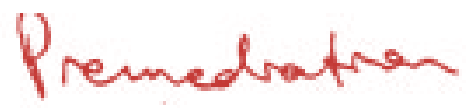

On the morning of December 10, 2003, I opened my Eudora in-box to find an unread e-mail message from a person I had never met, named Sonja Neef, with the intriguing subject line: 'Invitation'. Opening the e-mail, I was flattered to find a letter inviting me to be the keynote speaker at a conference on 'remediating handwriting' planned for Weimar the following June. The letter concluded conventionally with 'Best regards, Sonja Neef'. But because it was an e-mail letter, it lacked the conventional handwritten signature of a printed letter. Instead, beneath the customary signatory formulation on this electronic letter, which (like the letter itself) Dr. Neef had composed specifically for the purpose of inviting me to this conference, was another signature, her 'SIG file', which (like her return address at the top of the e-mail message) had already been composed before she began writing this letter, and was included as part of the automated formatting of her e-mail program. ${ }^{I}$ Underneath this SIG file was an icon indicating the presence of the attachment referred to in the body of the message, a document in Microsoft Word entitled 'ReMediating Handwriting'.

Although in 2003 there was nothing at all remarkable about such electronic epistolary details, in the context of a book on handwriting in an age of new media this unremarkability is worth considering. Ten years ago, an invitation like this one would most likely have arrived in the form of a word-processed and printed letter; twenty years ago it would have probably arrived as a typed letter; a century ago it would have arrived as a handwritten letter. Differences in the materiality of mediation aside, there is one thing that all three of these historically hypothetical letters would have had in common: they all would have come with a signature in Sonja Neef's hand. While the materiality of the medium for composing such a letter has changed in the past century from handwriting to typewriting to word- 
processing to e-mail, it is only with the advent of e-mail that we have witnessed a change in the materiality of the signature from a handwritten autograph to a premediated digital signature file. What are the implications of this change?

One obvious implication is temporal or sequential. In print or handwritten letters, the signature has, historically, come at the end of the process of composing the letter. The last thing that the letter writer writes, the signature marks the letter as an authentic expression of the signatory; it marks the letter as a communication underwritten or authorized by, or accountable to, the signatory. In e-mail the SIG file and return address that authorize and authenticate the communication are written prior to the composition of the letter; they are pre-mediated. In other words, unlike a conventional handwritten signature or return address, the SIG file and return address are already 'written' at the beginning of the process of composing an e-mail, upon the initiation of the command to compose a new message; indeed in some sense they constitute the medial or technological condition of possibility of an e-mail message. In e-mail the SIG file and return address exist prior to anything that the letter writer writes, says, or promises; the letter is marked as authorized by its sender before it is even composed. Of course, one might contend that it is only after the e-mail is written or sent, when the signatory gives the command to send the message, that the signature and return address are actualized. But the same is true of a hand-signed letter sent through the mail; clicking 'send' is only the digital equivalent of mailing the letter. Alternatively, one might counter that in some sense such temporal premediation has always been the case insofar as the epistolary conventions informing handwritten signatures have always pre-existed any specific instance of letter writing. This is undoubtedly true; letter writing has for centuries been a regularized and conventionalized genre. Nonetheless, the premediation of the signature and return address still constitutes a temporal and sequential reversal of the acts of writing a letter and signing it, a material remediation of the handwritten signature whose consequences should not be dismissed or overlooked.

In addition to this shift in the sequentiality of the signature and the letter, there is a difference in what we might call the 'content' between a handwritten signature and a SIG file. As famously cited by Jacques Derrida, in an essay to which my title alludes, J.L. Austin maintains that the handwritten signature functions as the guarantor of the authenticity or originality or uniqueness of one's written expression, standing in the absence of the writer in the same relation to the written utterance as a speaker's body does to the spoken utterance (Derrida 1984, 307-330). As the unique mark or index of the signatory's name, which itself has been seen by philosophers of language to bear an indexical relation to its reference, the handwritten signature does not refer to a group or class of individuals, but only to the particular individual who has done the signing. Derrida challenges Austin's belief that the signature is categorically distinct from other forms of writing. Austin would distinguish the signature (and similarly speech) from writing because writing is iterable and citational but a signature (or a spoken utterance) is not. Derrida, on the other hand, argues that iterability and citationality are the properties of all communicative acts, written or spoken. For Derrida speech acts and handwritten sig- 
natures are both forms of writing insofar as they are iterable and citable like any other written utterance; handwritten signatures do not provide any fundamentally different relation to their authors than do speech acts or any other forms of writing.

At the beginning of the 21st century, iterability and citationality are among the hallmarks of digital media, serving as sources both of creativity and of crisis in contemporary communication and culture - as evident in such practices as sampling, rip 'n' burn, P2P software, piracy, or the proliferation of computer worms and viruses. Unlike the supposed uniqueness and authenticity of the handwritten signature, the SIG file is theoretically infinitely reproducible. The sIg file functions to identify the writer's coordinates within a complex web of heterogeneous institutional, geographic, and telecommunication networks. The handwritten signature has a temporal, indexical function, with the graphic inscription attesting that the signatory had been present at the writing of the letter. The SIG file, on the other hand, has a spatial, locational function, attesting to the institutional position, geographical location, and telecommunicational coordinates of the signatory.

In this essay, I consider how something like the premediation of the signature in one's SIG file speaks to the issues raised by Derrida's critique of Austin. In other words, at the present historical moment, what does it mean for the premediated signature (as constituted precisely by the iterability, citationality, and alienabilty of digital mediation) to take the place of the handwritten signature (as a form of mediation indexically bound to the 'original' context of its performance as an event)? More broadly, I want to explore the ways in which the premediation of one's image, the audio-visual, digital remediation of the self, is beginning to stand along with, if not exactly eclipse, the signature as the juridical mark of one's intentionality and legal responsibility/accountability. I will argue throughout that the regime of handwriting as guarantor of conscious, legal, or political intention is making way for a regime of digital imaging. My aim is not to claim that digital remediation is somehow categorically distinct from writing, or is taking its place, but that writing and remediation alike share features of iterability, citationality, and alienability, even if these features manifest themselves differently in different technical media and at different cultural and historical moments.

Before moving into the body of my argument, I should say a couple of words about what I mean by 'premediation'. Premediation is a concept I have been developing to account for a particular aspect of the logic of remediation that Jay Bolter and I had overlooked at the end of the 1990s, an aspect of remediation that has been emerging for some time, but which has become much more acutely manifest after the shocking events of September 11, 2001. In a recent article, I have defined the threefold desire that motivates the logic of premediation at the beginning of the third millennium (Grusin 2004, 17-39). First, where remediation entailed the refashioning of prior media forms and technologies, premediation entails the cultural desire to remediate future media forms and technologies. This desire is quite visible in recent Hollywood cinema - in the vR-like 'wire' in Kathryne Bigelow's Strange Days; in the heterogeneous networked technology of pre-crime and precognition in Steven Spielberg's Minority Report; or in the high-powered telescope 
that allows one to literally see into the future in John Woo's Paycheck. Second, premediation entails the desire to remediate the future before it happens, the desire to never again let catastrophic events like those of 9/11 catch us unawares. This desire to avoid the catastrophic immediacy of watching a plane crash into the World Trade Center live on television, or the Twin Towers burning and collapsing, is evident in the global news media's increasing emphasis away from reporting on the present or recent past and toward pre-mediating events of the future, events which may or may not ever come to pass. Finally, the desire to premediate the future before it happens is accompanied by a desire to colonize the future by extending our networks of media technologies not only spatially across the globe and beyond, but also temporally into the future. This desire is manifest in the way that the heterogeneous networks of global capitalism work to ensure that the future is always so fully mediated by new media practices, forms, and technologies that it is unable to emerge into the present without having already been premediated in the past.

It is this latter sense of premediation that bears most directly on the issues raised in this essay, particularly insofar as I am interested in thinking through the implications of the fact that in electronic communication the signature is increasingly premediated before the letter or other document it is meant to endorse is even composed. To see the digital signature as premediated is not, I need to insist, to see it as a Baudrillardian simulacrum; despite their apparent affinities, premediation is not simulation. For Baudrillard the

logic of simulation... no longer has anything to do with a logic of facts and an order of reason. Simulation is characterized by a precession of the mod$e l$, of all the models based on the merest fact - the models come first, their circulation, orbital like that of the bomb, constitutes the genuine magnetic field of the event. The facts no longer have a specific trajectory, they are born at the intersection of models, a single fact can be engendered by all the models at once. (Baudrillard 1994, 16-17)

Although it might seem that what I am calling premediation is simply a restatement of Baudrillard's claim that the logic of simulation is marked by a 'precession of the model', in which 'the models come first' and 'their circulation... constitutes the genuine magnetic field of the event', this is not the case. Where I see the premediation of the future as inseparably connected with the remediation of the present and past, Baudrillard sees the precession of the model in the logic of simulation as marking a radical break with the 'logic of facts' or the 'order of reason' that marked human history prior to the advent of the logic of simulation. Baudrillardian simulation is always marked by the apocalyptic rhetoric of 'no longer': simulation 'no longer has anything to do with a logic of facts'; 'the facts no longer have a specific trajectory' (emphasis added). Unlike Baudrillard's logic of simulation, the concept of premediation imagines neither a world of total entropy or absolute mediation, an endless circulation without meaning, nor an absence of the real or its hyper-reality. Rather, in describing the way in which the future has already been pre-mediated, premediation conceives of this mediation as remediation, as always entailing 
the real in relation to prior media forms or previous media formations. In taking up the premediation of the signature at our current historical moment, I do not mean to celebrate or bemoan a radical break with past signatory practices - indeed I refuse to accept Baudrillard's notion of such a break. I mean instead to historicize both the present and the future in terms of their remediation of past signatory practices - in order to understand the specificity and particularity not only of the present moment's differences with the past but also of their continuities.

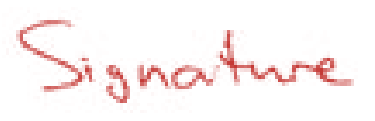

For some time now, commercially available software has allowed an image of one's handwritten signature to be incorporated into a printed/word-processed letter. Bill and Hilary Clinton, Robert F. Kennedy, Jr., Robert Redford, George W. Bush, and John Kerry are among those whose handwritten signatures (or, more accurately, printed versions of their signatures' digital images) have come to me through the mail in recent years. Electronically generated signatures on private and public sector bank checks are now nearly universal. Gone are the days when in 1971-72, as a young cashier at Merrill Lynch, Pierce, Fenner and Smith, my daily responsibilities included typing out in triplicate and co-signing by hand disbursement checks for the brokerage house's clients. Such checks are now generated and signed by computerized processes and nobody thinks anything of it. The same technology that incorporates an image of one's signature into an automated accounts payable system or mass-produced marketing letters from celebrities or other public figures, can now be used for electronically transmitted letters as well. So it is no longer unusual to receive an e-mail attachment in Microsoft Word that has a memo or letter with an appropriately placed digital image of the sender's signature.

Although the e-mail invitation I received from Sonja Neef in December 2003 did not bear her handwritten signature, it could have come with an image of that signature if she had so chosen. The technology to do so has existed for some time now and is readily available on the Web. Indeed, there are any number of different ways in which one can append a signature to one's e-mail or other electronic correspondence. Some websites offer to send you generic handwritten signatures to use as your e-mail's sig file. If you choose, you can have this generic signature animated. For a slightly larger fee, you can have your own signature scanned as a digital image and incorporated into your e-mail. And this signature, too, can be animated. While us and international legislation has made electronic signatures legally binding since October 1, 2000, one online purveyor of electronic signatures, LiveSignatures, denies that the products it sells can be considered legal signatures. In response to the first question on its FAQ page, which asks if it is safe to send one's LiveSignature via the Internet, LiveSignatures implies that because this signature is only an image of one's signature and not the signature itself, its customers should not worry about their digital signatures being stolen or misused by identity thieves because such signatures are not legally binding (see <http://www.livesignatures. com/faq-6.asp $>$ ). 
If a digital image of one's signature is not considered legally binding, what then would constitute a legal electronic signature? ESIGN, the Electronic Signatures in Global and National Commerce Act passed by the us Congress in 2001, legislates that a signature cannot be denied to be valid simply because it is in an electronic or digital format. ${ }^{2}$ Businesses and governments may implement electronic signature technologies that can, with certain exceptions, function in place of written signatures. Because of significant differences between the media of paper and electronic documents, however, ESIGN stipulates some interesting preconditions to the acceptance of electronic signatures, ostensibly with an eye towards protecting the consumer. The ESIGN act requires governments or businesses seeking to substitute electronic for paper documents to ensure that:

the consumer consents electronically, or confirms his or her consent electronically, in a manner that reasonably demonstrates that the consumer can access information in the electronic form that will be used to provide the information that is the subject of the consent. ('ESIGN',101(c)(1)(C)(ii))

Furthermore, the ESIGN act directed the us Department of Commerce and the Federal Trade Commission to conduct a study within 12 months of the passage of the bill on the benefits and burdens to consumers and businesses of the provisions set forth in the consumer protection sections ('ESIGN', 105(b)). This study was based largely on a public workshop consisting of 'moderated round table discussions with representatives from industry, government, consumer advocate groups and other interested parties'. ${ }^{3}$ Among the study's conclusions is that by requiring 'businesses to obtain from consumers electronic consent or confirmation to receive information electronically that a law requires to be in writing', the ESIGN act insists 'that the e-commerce business determine whether the consumer has the ability to receive an electronic notice before transmitting the legally required notices to the consumer' ('ESIGN Consumer Consent', III.A.I). According to several of the publicly solicited comments from the workshop, this consumer protection provision:

ensures that the consumer has access to a computer and to the Internet; ensures that the consumer has access to the software necessary to open the documents that are to be transmitted electronically; and raises the consumer's awareness of the importance of the documents received and the importance of receiving the documents electronically. ('ESIGN Consumer Consent', III.A. I)

As all of these comments make clear, the ESIGN Act marks a recognition by us government, industry, and consumer advocate groups that electronic commerce and electronic signatures cannot exist, nor can they have any legal or commercial efficacy, without having already been premediated. The requirement that consumers consent in advance to doing business electronically (or more accurately allowing themselves to sign electronically and receive legally mandated reports electronically) recognizes that there must be a premediated network in which the consumer 
participates and within which the consumer knows how to act. This confirmation must of course be in advance of the implementation of electronic signatures or delivery of electronic documents that the law already stipulates must be in writing. This legally required premediation does not, as Baudrillard might have it, do away with individual agency or meaning in favor of an endless circulation of digital mediation. On the contrary, it defines the terms and conditions through which juridical and economic agency function in our current historical moment.

The ESIGN Act is generally regarded as technologically neutral; it does not specify any particular technology that can or cannot count as an electronic signature, which it defines simply as any 'electronic sound, symbol, or process, attached to or logically associated with a contract or other record and executed or accepted by a person with the intent to sign the record' ('ESIGN',106 (5)). While this definition is vague enough that it could (under the right conditions) cover such digital images of signatures as those offered by online businesses like LiveSignatures, the ESIGN act has fostered the development and marketing of several different products to capitalize on these new possibilities, including digital certificates authorized by public key infrastructure (PKI), fingerprints, iris scans, and handwritten electronic signatures. All of these electronic signature technologies use algorithms to support some form of certification, encryption, and decoding; the latter three technologies all connect the electronic signature function to the presence of the human body at a particular place and time.

For the purposes of this essay, the most interesting of these technologies is, of course, electronic signature technology, which begins by capturing a handwritten signature via handwriting recognition software, then transforms the captured signature algorithmically into an encrypted file which is bound to the document, stored in a database, and able to be decrypted as visible signature at the end. Current technology not only stores the signature as an image, but also stores biometric data connected with the signature process to provide an extra element of security in guaranteeing that the signature was not forged by another person or otherwise faked. The deployment of biometric verification relies upon the assumption that written signatures are premediated in the body as something like an automatism, not unlike the way in which a sub-routine, or JavaScript, or embedded program can be premediated in a website or other digital artifact. The idea behind these biometric measurements, then, is that the body itself is a medium, or perhaps an engine of mediation; such practices or actions as signing one's name or writing by hand are understood to exist as habitual automatisms that, when triggered, operate in the same way every time to produce the same handwriting, the same signature. ${ }^{4}$ These automatisms are understood to be built into the body, not unlike the way that SIG files or return addresses, for example, are built into your e-mail program.

One us company that is aggressively marketing this new electronic signature technology is Interlink Electronics, which offers a Flash video on its website to tout its new E-Pad product line. 5 The voice-over on Interlink's marketing video dramatically demonstrates the way in which proponents of electronic signature technology seek to preserve the traditional role played by the handwritten signature even 
while capitalizing on the opportunities brought about by newly legalized electronic signatures, contending that: 'Interlink's E-Pad captures the traditionally accepted personal handwritten signature, biometrically transforming it for secure use in the digital domain' ('Electronic Transactions', 2005). The video opens with a more elaborate version of this double gesture, aiming both to preserve the historical role of the signature and to remediate it for the digital revolution:

As individual as DNA, the personal signature has been used for hundreds of years to close letters, finalize agreements, certify applications, and authorize transactions. Recent legislation, however, has sparked a revolution in the way personal signatures are used. Throughout most of the world electronic signatures are now considered to be as legally binding as those written on paper. E-PAD, from Interlink Electronics, is an electronic signature solution, that employs the time-tested handwritten signature, capturing and converting it to a biometrically secure e-signature, that once bound into an electronic document, cannot be removed, reused, or altered.

As one example of the persistence of the handwritten signature in an age of digital remediation, Interlink Electronic's new 'electronic signature solution' reflects the corporate and cultural desire that 'the digital domain' will hold on to the stability of an older techno-juridical regime in which handwriting is seen to have an unalienable relationship to identity.

Because of this supposedly inalienable relationship, which the Interlink video updates for the 21 st century by likening the uniqueness of one's signature to the uniqueness of one's DNA, signatures have played a key role in authorizing or guaranteeing one's legal or economic agency in modernity. Historical understandings of the exact nature of this relationship, however, have changed over the past two centuries or more. The Romantic sense in which handwriting is seen to reveal one's character by proceeding from the unconscious as an automatic gesture, has given way to the more modern sense in which the signature is connected physiologically (or now biometrically) to one's distinct, unique identity not as a representation of one's character but as an automatized guarantor of one's legal, contractual, economic agency (Thornton 1996, chapters 3 and 4). Although in electronic signature capture the distinctiveness of one's handwriting is authenticated by means of a series of networked information protocols, the signature continues to function as a unique marker of identity in these remediated electronic signature technologies. The handwritten signature is biometrically recorded so that electronic signature technologies can continue to provide assurance that the digitally encoded signature has not been stolen or hijacked or pirated by another. 


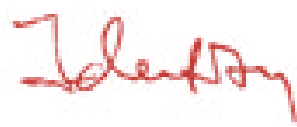

Legislation like the ESIGN Act (and its counterpart, the European Union Electronic Signatures Directive), as well as the technological and juridical practices of electronic signatures that have been developed in conjunction with such legislation, reflect the way in which the notion of the signature as an irreducible and inalienable marker of identity persists in an era of digital remediation. In us (and increasingly global) culture, however, there are many indications that this historical sense of stable identity is making way for a notion in which one's relation to one's identity is much more fluid and changeable, in which one's identity is coming to be marked not by one's handwritten signature but by one's digital image. And it is indeed precisely because digital images (like other digital mediations) are, unlike one's signature, in theory nearly infinitely reproducible and remediable, that technologies like electronic signature capture have been developed to authenticate contractual identity in a digital age.

The coexistence of these two different regimes of identity is plainly visible on the websites of the Motion Picture Association of America (MPAA) and the Recording Industry Association of America (RIAA), both of which include links to the two organizations' jointly authored 'Corporate Policy Guide to Copyright Use and Security on the Internet'. ${ }^{6}$

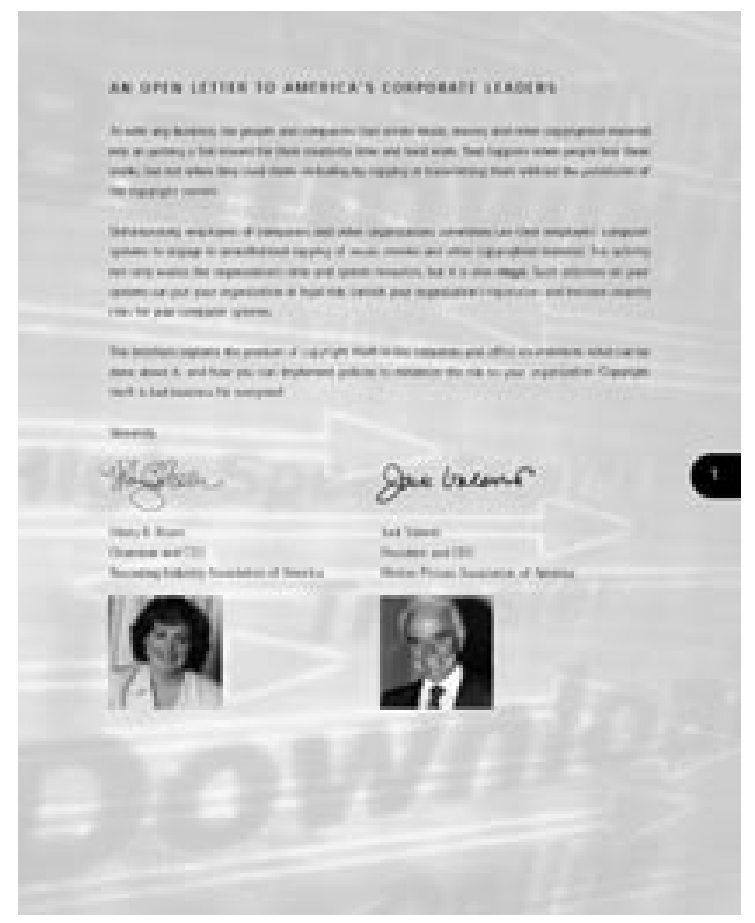


This policy guide is authorized and authenticated by an introductory 'Open Letter to America's Corporate Leaders' from Hilary B. Rosen, chairman and CEO of the RIAA, and Jack Valenti, president and CEO of the MPAA. The letter is signed by Rosen and Valenti, with their digitally reproduced photographs added underneath their names and titles. In their letter, the two ceos explain that the document addresses 'the problem of copyright theft in the corporate and office environment', a problem exacerbated by the fact that digitally encoded films and sound recordings can be illegally copied and reproduced without permission. The MPAA-RIAA document itself is presented as a PDF file, which cannot easily be sampled; for example, the photos of the two signatories cannot, as with most images on the web, be easily copied and pasted elsewhere, nor can the two signatures. Yet the Corporate Policy Guide also includes a sample memo to employees that is meant to be used by corporate management teams to put their own MPAA- and RIAA-sanctioned policies on use of copyrighted material into place; samples of such policies are also included in the online document.

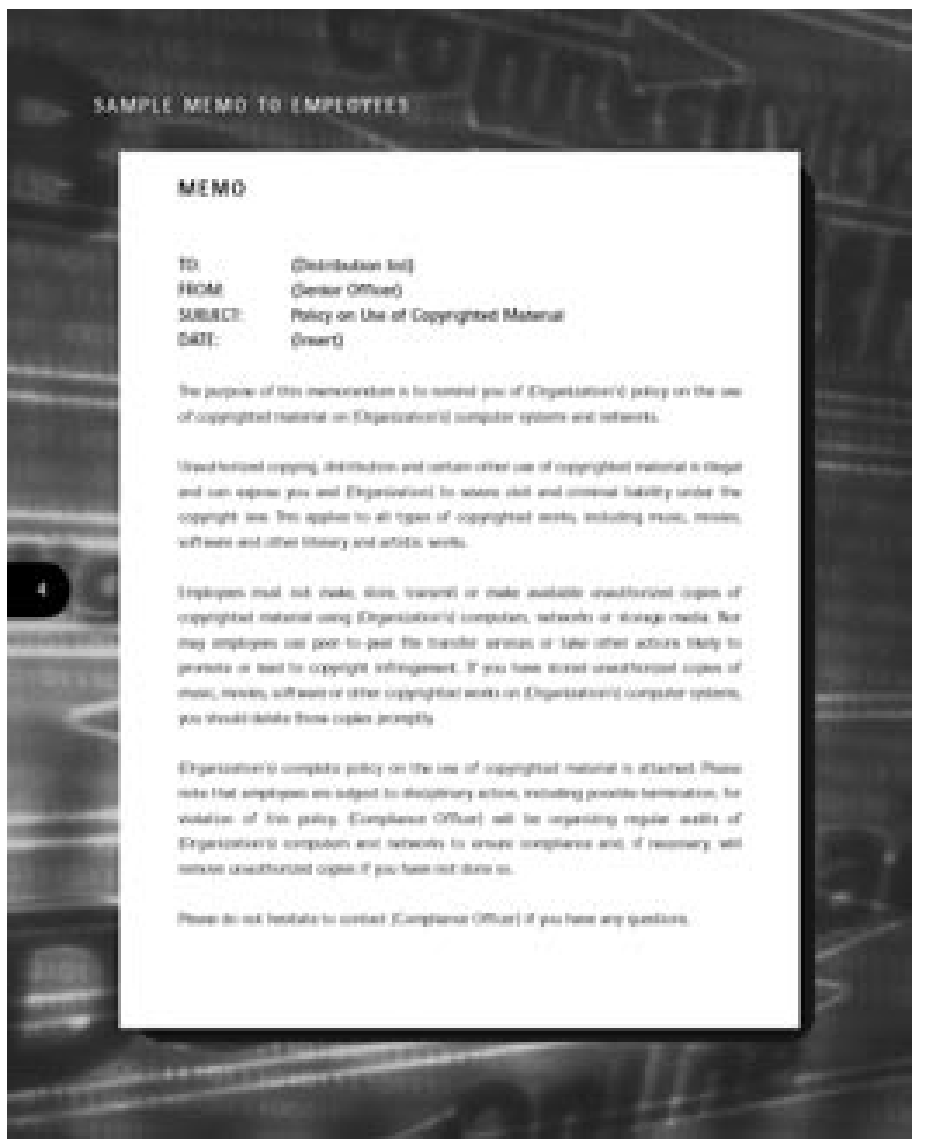


These sample memos and policies, unlike the copyrighted material they are meant to protect, are intended to be borrowed and appropriated, presumably without citation or acknowledgment, from the MPAA-RIAA document - even while being presented in a digital format (PDF) that makes such borrowing more difficult than if the sample memos and policies were available as downloadable Word files, for example. This joint document enacts and promotes one position on borrowing the work of others even while advocating another. And these two positions on expression - as the inalienable private property of its author and as sharable, reproducible common property - are enacted in the doubled 'signatures' of the authorizing letter.

The format of this letter is a fairly typical remediation of the format of the print letter, which adds to the digital images of the handwritten signatures digitized photographic images of the letter's two signatories as another kind of signature or authorization or identification. Indeed we find a very similar format in a letter on the Citigroup website, in which Sandy Weill, chairman of Citigroup, Inc., reports on the company's corporate citizenship efforts for 2003 (see May 23, 2005, <http:// www.citigroup.com/citizen/community/data/citizen03_en.pdf>).

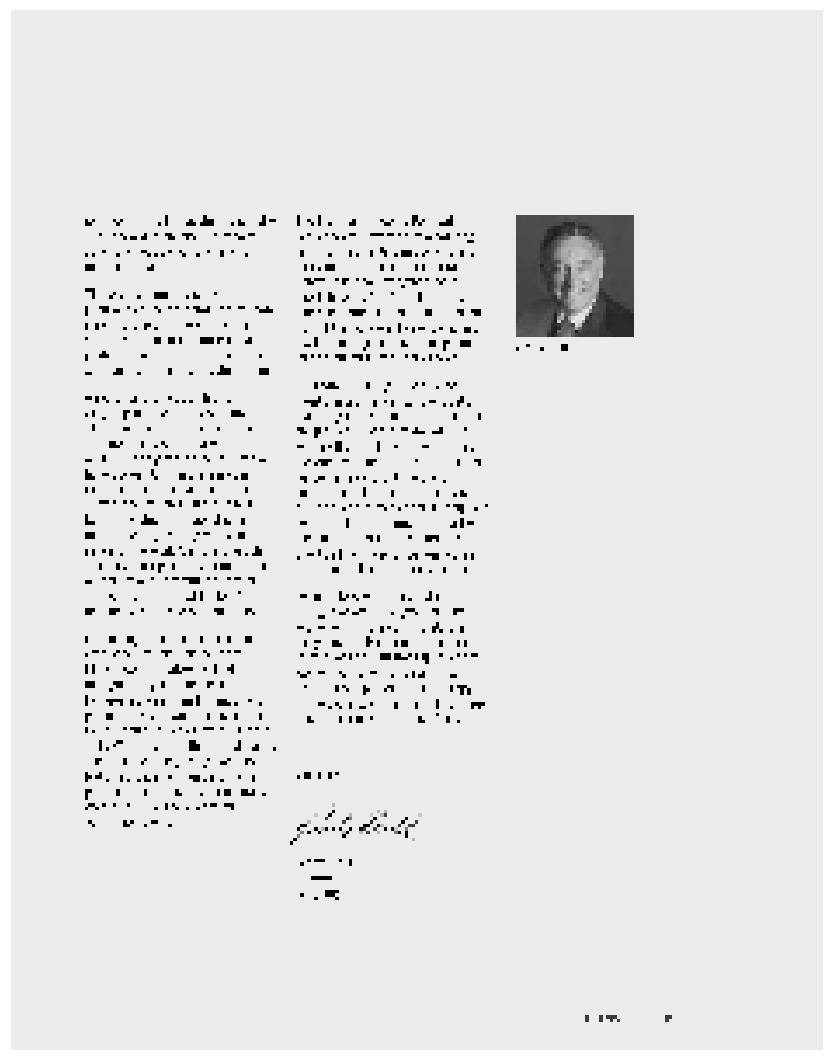


But what makes this joint MPAA-RIAA document particularly interesting is the way in which the open letter's supplementation of the signature with the digital image emblematizes the two regimes of copyright, authorship, or ownership that are contesting one another with regard to current digital media practices - an older regime in which identity is authorized or guaranteed by the uniqueness of the signature, and the current regime of digital remediation in which one's word, one's intellectual property, or one's identity, is guaranteed by means of the replicability (or iterability) of digital mediation. Like one's signature in an earlier regime, one's digital image is able to persist independently of the self to authorize one's action, expression, or communication. But because, unlike a handwritten signature, digital imagery is so easily reproducible and changeable, it poses a threat that corporate interests like those represented by the MPAA and RIAA are determined to resist.

These competing notions of identity in our age of digital remediation are very much at play in a 2004 Citibank ad campaign about identity theft, which cleverly links together identity theft with the theft of one's digital, audiovisual image. Aired primarily in the US, this series of television commercials represents the theft of one's identity not as the loss of one's financial information or the unauthorized access to one's digital data, but rather as the loss of control over one's audiovisual image, the hijacking or appropriation of one's body as if it were a puppet. In these commercials the video image of a person talking is accompanied by an audio track of the words of the person who has stolen their identity. In each case the two identities are cross-gendered. The ads also suggest other differences between the voice of the thief and the body of the victim in terms of age, class, race, and ethnicity. In one commercial, for example, we see a video of a young African-American woman, 'Sandra T.', seated in a beauty salon, drying her nails and soaking her feet in a Jacuzzi; the woman speaks to the camera, however, in the voice of a young white male nerd or geek, bragging about how he broke through her firewall, stole her account number, and purchased a 64 -inch screen plasma television and a 'girl robot' for his prom date. In another commercial, a working-class white man, Jake B., is sitting in a leather chair in what looks like his den or television room; he speaks to the viewer in the voice of a young woman with a New York accent, who brags about emptying his checking account, going to the mall, and buying 'a sexy little outfit', spending '\$1500 for a leather bustier' that 'lifts and separates'. In these Citibank commercials the metaphor of identity theft - the illegal use of another's financial, political, or legal information - is literalized. Identity theft is represented in these ads as stealing and manipulating somebody's mediated image, as one person making another person's self say what the first person wants, making one's self do (in terms of its function as a consumer) what another wants. Rather than depicting what might actually happen in case of identity theft, i.e., an identity thief acting in his or her own body as if he or she possessed the identity of another (consuming or spending or contracting as another), this commercial reverses the 'real' situation, and has the audiovisual image of the person whose identity has been stolen speaking in the voice of the identity thief, who is manipulating that person's body, not its information or its credit.7 Rather than presenting identity theft as the channeling of the victim's information through the thief's body and actions, these 
commercials present identity theft as a kind of body-snatching, a channeling of the thief's voice and actions through the body of the victim.

Fallon, the advertising agency responsible for this television campaign, followed it up with an analogous print campaign, in which still photographs of the victim of the identity theft are portrayed in the situations in which the identity thief has used their credit (see May 23, 2005. <http://www.magazine.org/advertising and_pib/kelly_awards/Winners_and_Finalists/11682.cfm>).
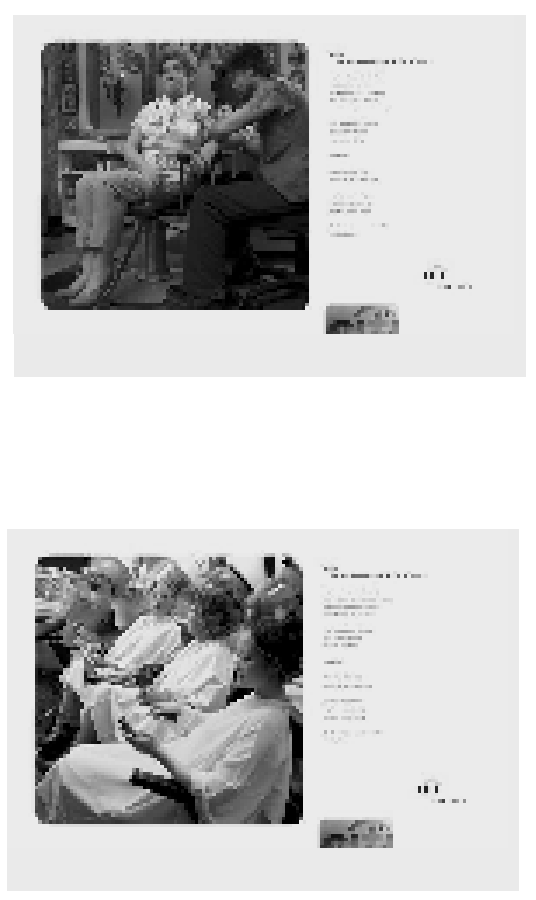
Rather than depend upon the shock value of an incongruity between body and voice, these print ads depend upon the shock value of the incongruity between the body of the victim and the action or situation in which he or she is placed. Thus one ad shows a middle-aged woman being given a tattoo in a tattoo parlor. A second presents a bearded, overweight man in a sleeveless gray T-shirt, holding a can of soda or beer, asleep under a hair drier in a beauty salon, next to three younger women. The tagline for each ad is 'It didn't seem right to us, either', emphasizing Citibank's 'early warning' protection, which looks out for purchases that don't match a profile of a user's purchasing history. Like the television commercials, these print ads work by reversing the identity theft situation, imagining that the identity thief manipulates not simply the victim's credit, but the victim's digital image as well, as if one's identity was in fact the sum total of one's financial information.

Where the Citibank ads seem chiefly concerned with the crime or scandal of manipulating another person's digital image, the 19th-century legal and economic system was preoccupied with the scandal of manipulating another's signature, and was thus largely concerned with the problem of preventing forgery, in order to maintain the economic-juridical legitimacy or uniqueness of one's signature (Thornton 1996, 101-103). In this earlier model, identity and voice or identity and body (like identity and handwriting), were thought to be inseparably tied together. In fact it is the (explicitly schematized) disjunction between voice and body in the Citibank television commercials that dramatizes identity theft; in so doing, the commercials depend for their force upon the conventional link between voice and body, as well as between voice and self. In this older notion of identity, the conjunction of voice, body, and self, like the identity between one's signature and one's self, authorized or attested to the legal responsibility of the rational individual in an Enlightenment-derived modern capitalist society. This traditional model of identity is now being challenged by new information technologies, which allow this bond to be severed, because one's identity is no longer authorized solely by one's voice or one's handwriting, but also (increasingly) by one's digital image, one's audio-visual remediation. Because this bond can so easily be severed, one's identity (as agent of cyber-capitalism, legally and economically responsible for one's words and actions) can more readily be counterfeited or stolen, and thus one must work to protect one's identity; to preserve the link between one's body and one's voice; between one's words and one's actions. While Citibank's ads portray the victims of identity theft as working or relaxing in their local, domestic contexts, the voices of the identity thieves are the voices of mobile consumers, whose chief crime is extravagant spending of a kind that cyber-capitalism has been structured to accommodate (and indeed to encourage - the identity thieves all seem to be having much more fun in these commercials than their puppet victims). These voices control (at least for the duration of the commercials or ads) both the bodies of the identity theft victims and their credit. This redefinition of identity as analogous to credit is a redefinition of identity as always already premediated, as the totality of one's financial and (by extension) other juridical or institutional information, not as the indissoluble connection to one's body via the voice or the hand. In these ads, iden- 
tity is imaged as embodiment even though it is identity as information that has been stolen.

Of course, as Katherine Hayles and others have reminded us, in the digital realm embodiment is information; the audio-visual representation of human bodies is made up of digital code or information (Hayles 1999). This linkage of identity and information informs the Citibank ads, in which the body, or its digitized audio-visual image, is 'forged' or 'stolen', or in certain respects 'played' as something like a character in a computer or video game. In these commercials, to become a victim of identity theft is in some sense to become an 'avatar' for the thief. Indeed, corporations like Citibank understand their customers to exist in relation to their juridico-economic identities in much the same way as game players relate to their avatars. Unlike the distinctive relation between handwriting and character or individuality that was historically imagined to run through the body and the hand, in our age of digital remediation the body (as digital image) is necessarily, by definition, alienable from one's self or identity - and one relates to it as a character or avatar one plays in a game and not as an indissoluble part or expression of self. These ads participate in a broader corporate media effort to displace or exaggerate the nature of the 'theft' to which consumers are vulnerable. Thus, while the mass news media regularly report on the nightmares of people like us having their identities stolen, the threat portrayed by identity theft (charging lots of purchases on another's credit card) is a minimal one - in the us each victim is legally liable for only $\$ 50$ per card. So, by equating this financial loss with loss of control of one's body and one's words to another, Citibank's commercials exaggerate the threat to its customers. Although identity theft is being portrayed exclusively as a threat to the individual consumer whose identity might be stolen, it is in fact equally or more substantially a threat to multinational finance companies like Citigroup. Thus, what is also being displaced in this ad is the relation between multinational corporations like Citigroup and its customers, which is, itself, also very much like a relation between a game-playing agent and its avatars. The unmentioned victims of 'identity theft' are corporations like Citibank itself, whose liability is greater in terms of both current and future economic costs because it has millions of dollars at stake in maintaining an e-commerce environment in which its customers feel secure.

Citibank's identity theft ad campaign portrays credit card fraud in terms of a shift from the fear that someone might forge your signature to the fear that someone might hijack and control your digital image. In the process, these commercials dramatize the way in which new digital media have begun to destabilize the historical relationship between one's handwritten signature and one's veracity, liability, or accountability. To say this, however, is not to deny the powerful way in which the signature still operates in political, legal, and cultural discourse. Signatures still function millions of times every day in legal, commercial, and social transactions. Signatures enact laws, close contracts, and authorize actions of innumerable kinds. Nor is it to claim that the instability of the handwritten signature is solely a phenomenon of our digital age. After all, signatures have always been able to be forged and thus have always been subject to challenge, in whatever medium they may oc- 
cur. Yet, despite (or perhaps in addition to) the ways in which the signature persists in an age of digital remediation, its challengability or revocability has been heightened in our current digital regime. As digital images replace signatures, what it means to authorize or authenticate one's words becomes less and less clear. The supplementation of the handwritten signature by the digital image appears to signal some kind of shift in the way in which human identity, and thus human accountability or responsibility, is currently understood. It is not (as Baudrillard might say) that the signature 'no longer' functions as it has for centuries now, in order to authorize or authenticate individual agency or responsibility, but that the signature (and all of its digital remediations) functions in a variety of media and formats that may not have been available in an earlier medial regime. Changes in the technologies through which identity and property are licensed, represented, and owned bring with them (or perhaps are brought about by) different understandings of the relationships among writing, self, identity, ownership, and property.

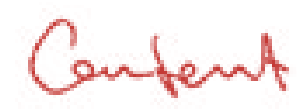

At least since Plato's 'Phaedrus', the question of writing has been tied up with the notion of memory. Indeed this is one of the underlying reasons for the legal efficacy of handwritten signatures, which are meant to stand as written records of an individual's intention that can persist beyond any one or more individual's memories of those intentions. Several recent Hollywood films have dramatized the changing conceptions of the self, not as an internalized essence or quality bearing an inalienable relationship to one's identity, but as a form of digital content, something alienable, made up of, or identified with, a set of mediated, or remediated, audiovisual images and sounds. In these films, memory is increasingly supplemented not only by writing, but also by images. One powerful example is Memento (2000), where the main character's short-term memory disorder forces him to use Polaroid snapshots with handwritten notes on the back of the photos to serve in lieu of his memory of people, places, or promises. A more light-hearted treatment of memory disorder can be found in the romantic comedy 50 First Dates (2004), in which a young woman cannot remember anything past the date on which she had a horrible car accident; at the end of the film her 'memory' is brought back to her each morning by a multimedia presentation that reintroduces her to her husband and daughter, both of whom post-date her memory loss.

The preoccupation with memory and identity also recurs in a number of recent mainstream Hollywood films that have premediated the idea of memory as constituted by a collection of audiovisual images that can be erased and stored by digital technologies. The Final Cut (2004), for example, premediates a society in which parents can choose to purchase a biotechnological memory chip to be implanted in their newborn child, which will record their child's entire life as first-person pointof-view audiovisual content. And Eternal Sunshine of the Spotless Mind (2004) premediates a different kind of future media technology, one that can be used not to preserve but to erase memories, usually those of a beloved person (or a pet). Al- 
though The Final Cut premediates a technology that seeks to preserve memories after death and while Eternal Sunshine premediates a technology that would erase memories during life, both participate in the current Hollywood project to imagine memory and therefore identity as a kind of biotechnological, networked, audiovisual content.

Perhaps the most interesting of these recent Hollywood films is John Woo's Paycheck (2003), in which the question of memory as audiovisual content is also at play. Significantly, the question of memory is here tied directly to the relation between signature and identity. Based on a story by Philip K. Dick, Paycheck concerns a software engineer named Michael Jennings (played by Ben Affleck) who uses reverse engineering to pirate other companies' proprietary technologies, in exchange for a large paycheck, hence the title. To protect his employers, however, he signs an agreement prior to commencing each job to have his memory erased from the day on which he receives the paycheck for his completed work to the day before he signed his contract. The film's plot is built upon disjunctions between his erased memory and several instances in which his intentionality is expressed by his signature, contrary to the memories that he retains.

The first disjunction occurs when Jennings goes to collect on his payment for an unusually long three-year job, stock options worth upwards of $\$ 92$ million. When he arrives at the financial institution to determine how to invest his earnings, he is told that he had voluntarily signed away his stock options four weeks earlier in exchange for an envelope of miscellaneous, apparently random, personal items. When Jennings (after having his memory wiped according to the contract he signed before beginning the job), discovers that he has somehow been tricked or swindled, that he has signed away his rights to the multi-million dollar payoff in stock options in exchange for an envelope of worthless personal possessions that he does not even recognize as his own, the point that the film underscores is that even in the hypermediated society of the near future signatures abide, that signatures, not memory, have legal (as well as cinematic) force. Although the audience is clearly led to believe, as Jennings himself does, that he has been swindled, that somebody must have signed his name or made him sign his name against his will or intention, it becomes evident well before the end of the film that his signature in fact does signify his intentionality or purposefulness irrespective of apparent logic or reason, or of his memory (or lack thereof). More precisely, however, what the audience (and Jennings) eventually discover is that this signature-authorized intention constitutes a premediation of his future actions that provides him with the clues and the necessary tools that will allow him to escape his pursuers and ultimately to destroy the dangerous technology he has just helped to create through reverse engineering.

The second disjunction between Jennings' signature and his memory occurs shortly after he discovers that he had signed away his paycheck, when two FBI agents apprehend him for patent applications he had taken out on technologies that had been illegally stolen from the federal government, applications that were authorized by his signature. 'These are patent applications', they tell him, as the screen shows a digital image of a government form. 'Look whose signature is on 
these documents'. The technology for which Jennings had taken out a patent, and which he was then determined to destroy, was a technology literally to pre-mediate the future, to see the future before it becomes reality. This technology is described by the two FBI agents as a visual laser powerful enough for its user to look around the curvature of the universe so completely that he ends up looking back at himself, not in the present or past, but in the future. Despite its fascination with premediation, PAYCHECK presents this futuristic technology as a dangerous threat that must be done away with. In so doing, the film ends up maintaining the value of the signature as a genuine expression of self, of intentionality, as against a notion of the self as remediated audiovisual content. Indeed, not only does Jennings succeed in destroying this technology, but, in typical Hollywood fashion, he ends up getting his fortune as well, by having 'seen' the lottery numbers of a future drawing, whose winning ticket is one of the items he has left himself in the envelope.

In PAYCHECK the plot elements involving Jennings' signature depend on the legal force of his signature to affirm his intention, independent of his memory or (in the case of his $\$ 92$ million) of any apparent logic or reason. Additionally, the invocation of patents can hardly be accidental, as they are precisely part of the older regime of copyright and intellectual property that is currently being challenged by new digital media technologies and practices. Paycheck thus dramatizes the conflict between the two competing regimes of identity and memory at play in contemporary culture. Although on the one hand, this film characterizes human identity as a kind of media content, made up of alienable memories and experiences that can be erased from one's memory, by the end of the film it turns out that the intentionality authorized by the signature that gives up the stock options is finally fulfilled, insofar as the items that Michael Jennings receives in exchange for them, prove necessary for him to destroy the technology he had created and to receive even more money than he would have received from the stock options. Despite its fascination with the premediation of future technologies and the portrayal of memory as audiovisual content fundamentally alienable from identity, Paycheck finally wants to hold on to an earlier regime of handwriting as key to enforcing or authorizing the intentions of the self independent either of memory or against the audiovisual premediation of the future. Like The Final Cut and Eternal Sunshine, Paycheck participates in Hollywood's (and in a broader sense, multinational capitalism's) double stance towards the cultural practice of remediating identity as digital, or audiovisual, content - a fascination with the idea and with its technological manifestation at the same time that an older notion of the self ends up governing these films' narratives.

I want to conclude, then, by raising some broader questions that need to be explored concerning this double stance towards, or contradictory role of, individual responsibility or accountability in contemporary culture. Does the proliferation of different technologies for authenticating identity in social, cultural, interpersonal, legal, commercial exchange bring with it new and significant implications for our understanding of property, of responsibility, of identity, and other practices that we consider legal or ethical or appropriate? Are ideas of an autonomous individual, of legal rights and responsibilities, of rationalized, consistent positions, of pri- 
vate property, rooted in an outmoded Enlightenment notion of self, private property, or freedom, that is bound up with older technologies of writing or mediation, and thus less viable under our current medial regime? Have such ideas, if not perhaps eliminated completely or replaced, been eroded by the emergence of another model, a model of networked identity and action and events in which the self does not exist prior to its actions, and in which the self is not accountable as the source or guarantor of its actions, but rather exists only as the product of an already premediated network of possible actions? Does the desire to premediate the future, to anticipate structurally all possible futures, many of which will never come about, do away with, or at the very least minimize, the consequences of being contradictory or untruthful?

In an age of digital mediation, my answer to these questions and others like them is inevitably doubled. On the one hand, I feel that the changes in the nature of the signature that I have articulated here, the supplementation of the handwritten signature by the premediated digital image, mark something fundamentally different about the way in which identity functions under the regime of digital mediation. On the other hand, I do not want to be seen as arguing that this difference marks the apocalyptic end of a stable regime of individual agency and responsibility. Instead, I cannot help but suspect that what we are now witnessing is only the latest chapter in an ongoing and constantly changing historical relationship among signatures, identities, and their multiple remediations.

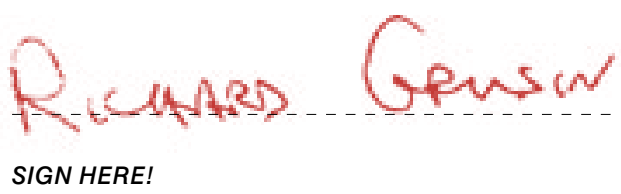


Notes

1. A SIG file is defined as 'A short block of text at the end of a message identifying the sender and providing additional information about them.... A .sig file is [a] small text file (with a .sig extension) that can be automatically attached to the end of email messages. The phrase SIG file is also used to identify blocks of text used for similar purposes through different channels, such as discussion group messages. For personal use, SIG files often include humorous sayings or signature art. These can be automatically rotated so frequent recipients do not see the same message every time. For business use, SIG files often include a mix of contact information and business promotion. This may include the sender's name, job title, company name, phone \#, fax \#, email address, Web site address, tag line and brief benefits of your products or services.' MarketingTerms. com. May 16, 2005. Available at: <http:// www.marketingterms.com/dictionary/sig_ file/>.

2. 'Electronic Signatures in Global and National Commerce Act.' Pub. L. No. 106229, 114 Stat. 464, 2000 (codified at 15 U.S.C. § 7001 et seq.). All references to this act will be cited parenthetically by section number in the text as 'ESIGN'.

3. 'Electronic Signatures in Global and National Commerce Act, The Consumer Consent Provision in Section 101 (c) (1) (C) (ii).' Pub. L. No. 106-229, 114 Stat. 464, Appendix D, 2000 (codified at 15 U.S.C.
$\S 7001$ et seq.). All subsequent references to this publication will be cited parenthetically in the text as 'ESIGN Consumer Consent'.

4. For further discussion of the body as medium, see Bolter, Jay David, Remediation: Understanding New Media. Cambridge: MIT Press, 1999, 236-240.

5. Interlink Electronics is a publicly traded company (Nasdaq: LINK) which describes itself on its home page as 'a world leader in the design and manufacture of intuitive interface technologies and products'. May 23, 2005. Available at: <http://www.interlinkelec.com/] >.

6. When this address was written in May 2004, the document was linked to both organizations' home pages. The MPAA link was still on its home page as of May 2005 at <http://mpaa.org/anti-piracy/ press/2003/2003_02_13.pdf > ; last accessed May 23, 2005. As of May 23, 2005, the RIAA link is buried on its press page: $<$ http://www.riaa.com/news/newsletter $\backslash 021303 . a s p>$ and the link to the joint document is broken: <www.riaa.com/pdf/ brochure2003.pdf $>$.

7. The idea of identity as a form of what I would call 'premediated' credit is one that Derrida explored in a number of places, most recently in his work on the impossibility of the gift. See especially Given Time: 1. Counterfeit Money Trans. Peggy Kamuf, Chicago: University of Chicago Press, 1992.

\section{Works cited}

Baudrillard, Jean. 'The Precession of Simulacra', in: Simulacra and Simulation. Trans. She la Faria Glaser, Ann Arbor: University of Michigan Press, 1994.

Bolter, Jay David. Remediation: Understanding New Media. Cambridge: MIт Press, 1999.

Derrida, Jacques. Given Time: 1. Counterfeit Money Trans. Peggy Kamuf. Chicago: University of Chicago Press, 1992.

- 'Signature Event Context', in: Margins of Philosophy. Trans. Alan Bass. Chicago: University of Chicago Press, reprint edition, 1984.

'Electronic Signatures in Global and National Commerce Act'. Pub. L. No. 106-229, 114 Stat. 464, 2000 (codified at 15 U.S.C. $\$ 7001$ et seq.).

'Electronic Signatures in Global and National Commerce Act. The Consumer Consent Provision in Section 101 (c) (1) (C) (ii)'. Pub. L. No. 106-229, 114 Stat. 464, Appendix D, 2000 (codified at 15 U.S.C. $\$ 7001$ et seq.).

'Electronic Transactions flash movie from Interlink', May 23, 2005. Available at: <http:// www.interlinkelec.com/flash/etmovie center.html>.

Grusin, Richard. 'Premediation', in: Criticism vol. 46, no. 1 (Winter 2004). 
Hayles, N. Katherine. How We Became Posthuman: Virtual Bodies in Cybernetics, Literature, and Informatics. Chicago: University of Chicago Press, 1999.

$<$ http://www.citigroup.com/citizen/community/data/citizen03_en.pdf>.

$<$ http://www.livesignatures.com/faq-6.asp >. Last accessed November 15, 2004.

$<$ http://www.marketingterms.com/dictionary/sig_file/>.

$<$ http://mpaa.org/anti-piracy/press/2003/2003_02_13.pdf>.

$<$ http://www.riaa.com/newslnewsletterl021303.asp >.

$<$ www.riaa.com/pdf/brochure2003.pdf>.

Thornton, Tamara Plakins. Handwriting in America: A Cultural History. New Haven and London: Yale University Press, 1996. 


\section{Writing the Self: Of Diaries and Weblogs}

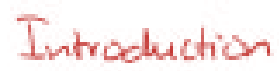

A cartoon that recently appeared in a Dutch newspaper shows a man and a woman lying in bed, smoking a cigarette apparently after having sex. 'Do you keep a diary?' the man asks his partner, and upon her response of 'no', he comments: 'Good. I don't like it when a woman immortalizes her intimate experiences with me on paper'. In the last frame, we see the woman sitting behind a computer screen and typing 'Dear weblog...', while the man snores away on the bed behind her. In this short cartoon, we can detect a number of preconceived notions about diaries and weblogs, but the clue to this joke is the paradox that the weblog is not considered a digital equivalent of the diary and yet it is. ${ }^{I}$

For centuries, the diary has been characterized as a private, handwritten document that chronicles the experiences, observations, and reflections of a single person at the moment of inscription. Although the diary as a cultural form is varied and heterogeneous, it typically represents the record of an 'I' who constructs a view of him/herself in connection to the world at large. Diary writing, as a quotidian cultural practice, involves reflection and expression; it is also a peculiarly hybrid act of communication, always intended for private use, yet often betraying an awareness of its potential to be read by others. Inviting the translation from thoughts into words via the technologies of pen and paper, the old-fashioned diary symbolized a safe haven for a person's most private thoughts. Personal notebooks were often treasured as stilled moments of a forlorn past, and kept in safe places to be retrieved many years later - much like photographs. But what has happened to the diary as we enter the age of digital technologies?

With digitization affecting practically every domain of public and private life, the diary is no exception. 'Weblogs' have become a popular genre on the Internet, as millions of people (particularly teenagers and young adults) are now heavily engaged in the activity of 'blogging'. By the end of 2004, there were about 10 million weblog users in the United States alone. ${ }^{2}$ But can weblogs and blogging be consid- 
ered the digital counterpart of what used to be a paper diary and diary writing? As the cartoon implies, the answer to this question is a paradoxical 'yes and no'. Cultural practices or forms never simply adapt to new technological conditions, but always inherently change along with the technologies and the potentialities of their use. In the case of weblogs, the digital materiality of the Internet engenders a new type of reflection and communication that shows traces of the former analogue genre, but also functions substantially differently.

Richard Grusin and Jay Bolter have used the term 'remediation' to account for the ways in which new media forms consolidate but also change existing forms (Bolter and Grusin 1999). In a critique of this term, Andreas Kitzmann argues that 'remediation' does not sufficiently account for the intrinsic shaping power of technology, and proposes to focus on the wider phenomenon of 'material complexification' to understand the continuities and changes between old and new media, for instance weblogs and webcams (Kitzmann 2003, 48-65).3 Both Bolter and Grusin and Kitzmann have a point. But I would argue that studying media changes is not a question of either cultural form or technology. In this chapter, I suggest examining not two but three dimensions of mediated cultural change: the materiality and technology of (hand)written diaries versus weblogs, the diary or weblog as a cultural form or genre, and the cultural practice of diary writing in comparison to the activities of so-called bloggers. While tracing the transformation of personal records in the face of new digital technologies, I will argue that weblogs are not outcomes but rather signifiers of cultural change as they both reflect and construct new epistemologies.

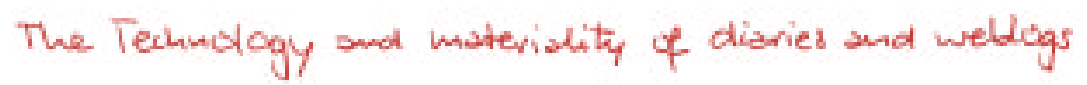

Diaries are commonly valued for their contents rather than for their look or feel. Nevertheless, the materiality of diaries as well as the technology through which these artifacts have come into being are crucial factors in their signification. ${ }^{4}$ Two typical concepts of diaries spring to mind: the empty diary, preformatted for daily use, which we can buy in stationary stores; and the original manuscripts of private diaries which have later appeared in print. The physical appearance of a prefab diary prefigures the functions of its intended use: empty pages, with or without lines, bound or unbound, dated or undated, offer the author stimuli to fill the more or less blank surface with personal inscriptions and thoughts. In some cases, the diary is completed by a lock and key - a potent symbol of its private nature. The preformatted diary has always been, to some extent, a product of contemporary fashion, its design and layout representing a particular style and catering to a specific age or taste. A diary's materiality forms an essential part of its content: pages, cover, key, colors, ink and paper (its look, feel, and smell) are all part of the act of memory. Over the years, diarists often grow fond of the material look of their notebooks fading colors, youthful handwriting, and ink blobs trigger reminiscences in a way that photographs might. The diary's contents, when reread at a later stage in life, may either elicit nostalgic yearning or retroactive embarrassment, in some cases 
even leading to a definitive destruction of the object. A reified memory object of one's past, the diary is the stilled result of a creative and communicative act.

Diary writing is not necessarily inspired by prefab formats: on the contrary, many diaries which were later discovered and subsequently published in print, had first been written in ordinary notebooks or scribbled on individual sheets of paper. The actual manuscript of such a diary, its original form of inscription, becomes a vital sign of authenticity - often stored in special places and only accessible to owners or researchers. In the case of Anne Frank's diary, which consisted partly of notebooks and partly of separate sheets of paper, the gradual discovery and reconstruction of the various 'versions' of the manuscript became part of the Dutch teenager's legacy. 5 The original manuscript, stored in Amsterdam, appeared to be in such demand that the Anne Frank Foundation had two exact duplicates made: one to replace the original on display at the museum, the other to satisfy the many requests from film directors, researchers and documentary makers for pictures of the original. The materiality of the manuscript constitutes an intricate part of the diary's genesis and, later, its controversial claims to authenticity, (uncensored) originality and completeness.

Pivotal to the materiality of diaries, up to the age of computers, has been the notion of script: the concept of a diary is commonly associated with (hand)writing, signifying not just authenticity, but personality. Handwriting has historically been believed to betray the personality of its producer - graphology being the study that yields clues to the writer's character such as age and even personality traits. Regarded as the first 'technologizing of the word', the availability of pen and paper facilitated the need to make oneself legible to the 'other' or to the future self (Ong 1982). Writing is thus intimately tied to a stage in one's personal development: a teenager's scrawls betray his or her inexperience with the prime tool of literacy the immaturity of body or mind. ${ }^{6}$ As Sonja Neef argues, handwriting is an embodied practice: moving a pen onto paper involves a direct connection between body and script, an act in which the eye and hand are intimately interwoven with the technology of paper and pen and the techniques of deploying them; the hand - a body part instrumental to the 'Verkörperung' (embodiment) of thoughts - fixes the inner self to the outside world (Neef 2002). ${ }^{7}$

Since other technologies have gradually replaced handwriting, the tools of a diarist have changed accordingly. When Sigmund Freud wrote his essay 'A Note Upon the "Mystic Writing Pad"', in 1925, he regarded writing and technology as external aids or supplements to memory. Freud described memory in terms of writing, comparing it to the surface of a writing pad that allowed the scribbling of notes that could subsequently be erased and yet remain stored in the 'subconscious' layers of the pad, below its material surface. Jacques Derrida, commenting upon Freud's essay, dismisses his notion of writing as an external memory and emphasizes instead technology's instrumental relationship to language and representation (Derrida 1995). ${ }^{8}$ Technologies, including writing utensils, are machines that engender representations while infiltrating agency. Pen and paper, therefore, produce different modes of writing than the typewriter or the word processor. ${ }^{9}$ Handwriting never simply structures reflections or thoughts, but literally creates them; 
by the same token, a typewriter constitutes a different relation between author, words, and representation. It may not be a coincidence that typewriters never became popular in connection with diary writing; unlike handwriting, the noise of fingers pounding on a machine severed the physical intimacy between body and word. ${ }^{\text {Io }}$ As the technologies for writing change, so does our way of creating self-reflective records. Handwritten diaries are material artifacts that are themselves memorials - traces of a past self. Memory, in other words, is always implicated in the act and technology of writing.

The advent of the personal word processor, as the successor to the (electronic) typewriter, further disembodied the production of written language, as not only the keyboard but also the screen interfered with the continuity between hand and words. Yet two essential features of word processing may have restored some of the intimacy lost with the typewriter. First, the relative silence of word processors refurbished part of the quietude inherent in solitary writing, while speeding up the production of text and maintaining standardized letter output. Even more profound has been the ability of word processors to produce tentative texts, provisional versions of thoughts, forever amenable to changes of mind; the editing of visualized words does not leave a trace in the ultimate print. Words on the screen, stored in digital memory, thus formed a new stage in the trajectory between immaterial thoughts and textual products, allowing for invisible revisionist interferences in one's memory. On top of that, digital files may never materialize into print, and they can remain stored in the black box of the personal computer, without ever being erased or retrieved (by the writer or by others). Diaries produced by a word processor, therefore, are fundamentally different from diaries produced by means of handwriting or typewriters because the personal computer provides an intrinsic textual paintbrush with which to edit one's personal records. The potential of digital editing at a later stage diluted the concept of the diary as a material, 'authentic' artifact, inscribed in time and on paper.

In the 1990s, when individual word processors gradually gave way to networked computers and the Internet became a popular medium for interaction, the diary seemed a doomed genre, incommensurable with the prime demands of ubiquitous connection rooted in digital materiality. The evanescence of the Internet appears at odds with the genre preference for a fixed material output. Moreover, the private nature of diary writing intrinsically conflicts with the connectedness of the electronic superhighway. Between body and words on paper there is no longer just a piece of equipment but a global network of connected individuals and communities. Such technological machinery is likely to hinder, rather than benefit, the privacy and intimacy of self-reflexive writing. And yet, perhaps surprisingly, the web$\log$ is one of the most popular genres booming on the Internet today. ${ }^{I I}$ The weblog appears, at first sight, to be a digital descendant of the paper diary, except that there is no printed output, only a screen-based one; since computers do not smell, and the screen has no particular feel, how can we define what the digital matter of weblogs actually consists of? 
Analogous to the preformatted paper diary and the diarist's handwriting, we can locate the materiality of weblogs in two different areas: weblog software and the signature of its users. Weblogs or webdiaries emerged in 1996, but only in the past four years has their popularity soared. Initially, blogs were typically personal websites operated by individuals who compiled chronological lists of links, interspersed with information and editorialized and personal asides. ${ }^{I 2}$ They later became experiments in self-expression, creating blog-communities where people read each other's weblogs and cross-linked their personal websites. The first weblogs were operated mostly by digirati, but as specially developed software made blogging technically easy, more people without any specific technological skills joined the various kinds of 'blogging groups'. ${ }^{\text {I3 }}$ Since 2000, a large number of software packages have flooded the market, enabling even the clumsiest person to become a sophisticated blogger. Today, users can choose from a variety of different packages, aside from open diaries on the web, such as Opendiary.com and MyDearDiary.com, there are also weblog services for which you need to sign up or even be introduced by a member, like LiveJournal, Blurty, Xanga, DeadJournal, Blogger, and DiaryLand. The formats may differ in layout and digital possibilities, but they all basically serve the same purpose. ${ }^{I 4}$ To some extent, these different designs resemble the preformatted paper diaries for sale at stationary stores. Various software formats attract different crowds, catering to heterogeneous tastes and lifestyles among teenagers, much like the brand names of fashion products. Weblogs are also dynamic material artifacts; they are not meant to be printed out and their contents are constantly evolving. Actually, a blogger may decide to leave its content exactly as it was first posted (and post additional comments) but of course the possibilities for editing, erasing, storing, cutting, and pasting are endless.

Software, however, merely constitutes the technological condition for its varied individualized use. Digital weblogs may, in terms of their materiality, not even remotely resemble their paper precursor, but there is a distinct continuity in their personal signature. If handwriting betrayed a diary writer's character and level of maturity, the typewriter and later the word processor had already erased that trademark of personality, and yet, through word choice, style, punctuation, and the use of emoticons it is remarkable how much the entries give away a person's character. On top of that, the personality of a diarist is even more traceable by means of her prolific choices of cultural contents; the blogosphere is part of a vast reservoir of texts, visuals and sounds, and most software packages support their inclusion via links or sidebars (e.g., to newspapers, fan sites, discussion groups, political lists, etc.). ${ }^{I 5}$ Weblogs seem particularly suited to accommodate the needs of teenagers, whose growth into adulthood is often characterized by quickly evolving loyalties to styles, fashions, and brands. Despite prescriptive software formats, weblogs offer a relatively high degree of creative freedom; users can discover their own taste by cutting, pasting, and commenting, thus exploring the relationship between the self and culture at large. Some weblog software (like OpenDiary.com) allows users to search entries by age group, gender, theme of the week, subject or cultural preferences. 
Although the multimedia weblog looks very different from the preformatted lockand-key paper diary, each materiality gives away clues to an author's personality. Just as paper diaries reflected someone's age, taste, and preference at a particular moment in one's life, the software and signature of blogs seem to accommodate the needs of contemporary teens and young adults to express and sort out their identity in an increasingly wired, mediated world. But technology does not tell the whole story. In conjunction with changing technologies and materialities, we need to pay closer attention to the cultural forms and practices of diary writing, to see how they change along with evolving notions of intimacy, privacy and memory.

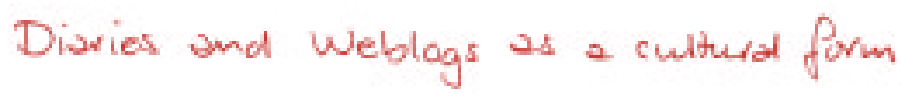

Another paradox surfacing in the cartoon discussed at the beginning of this article, is the idea that diaries are a strictly private genre, whereas weblogs are private yet open to all who want to read. Both assumptions are incorrect. Over the past centuries, the diary as a cultural form has been defined in a number of ways: the diary as therapy or self-help, as a means of confession, as a chronicle of adventurous journeys (both spiritual and physical), or as a scrapbook for creative endeavors. ${ }^{16}$ Beatrice Didier, a French literary theorist, articulates a more general distinction, based on the content of the entries, between the personal or private 'diary' ('le journal intime') and the more public or factual 'journal' (Didier 1976). Another French literary scholar, Eric Marty, classifies diaries by their addressees: are they strictly secret or written for others as well? (Marty 1985). In general, the taxonomy of the oldfashioned paper diary tends to be based either on its contents (personal, intimate self-expressions vis-à-vis daily records of fact) or on its directionality (intended for private reading vis-à-vis public use). But how useful is this genre classification along the axes of self and others, of intimacy and openness? And how does this distinction hold up in the face of new digital cultural forms and publishing practises?

The myth that the diary is a private genre, strictly written for oneself, is as misleading as it is persistent. A binary distinction between the diary as a personal record written for private purposes in contrast to a journal of fact written to show others, is hardly tenable. ${ }^{I 7}$ As Thomas Mallon argues, no one ever kept a diary just for himself; pointing out the continuity between the 'journal' and the 'diary', he concludes that both are directed towards an audience and 'both [are] rooted in the idea of dailiness, but perhaps because of the journal's links to the newspaper trade and diary's to 'dear', the latter seems more intimate than the former' (Mallon 1984, p. xvi). Of all the varieties within the genre, some diaries are written with a reader in mind more than others, but an essential feature of all diaries is their addressee. Whereas some authors directed their diaries to an imagined friend (like Anne Frank's 'Kitty', or André Gide's mysterious addressee), to God, or to the world-atlarge, the notion of addressing is crucial to the recognition of diary writing as an act of communication. ${ }^{18}$ Writing, even as a form of self-expression, signals the need to 
connect, either to someone or something else, or to oneself later in life. William M. Decker, who theorized the evolution of epistolary writing in the United States, observes that letters, much like diaries, carry the aura of a private genre, whereas the genre encodes itself according to public standards: 'What we identify as the private life is a conventionalized and hence public construction' (Decker 1998, 6). Diary writing is, to a large extent, a cultural form firmly rooted in rhetorical conventions: intimacy and privacy are effects rather than intrinsic features of the genre.

Another paradox we can trace in the genealogy of diaries is the belief that their creation is usually associated with individual voice and authorship, whereas in reality the genre has often been deployed as a communal means of expressing and remembering. To many religious congregations, for instance, the diary was a semi-public record, shared within but never outside of a community. Elizabeth Yakel describes in her intriguing account of the Maryknoll Sisters' archive how, between 1912 and 1967, this religious community adapted the genre as a collective means of expression to record and exchange spiritual and intellectual journeys to each other (Yakel 2003, 142-150). Their record-keeping practices suited various goals, from expressing individual beliefs to communicating information across time and space with like-minded congregations: 'The diaries had multiple audiences - they were a means of internal communication within the community and also served as a mechanism for external communication to Catholics and others interested in their mission activities' (Yakel 2003, 143). In the history of diary writing, the genre as a communal means of expression has found many practitioners, from South Pole explorers keeping logbooks to Pows writing their war diaries while held in captivity. As Michael Piggott, archivist at the University of Melbourne discovered, Australian archives contain many such collective ego documents, chronicling important episodes from the 16th to the 19th centuries through the eyes of transient groups (Piggott 2003, 68-75). For many groups bound together by an adventurous ordeal, a joint diary was a means to trust one's personal emotions to a relatively safe medium and share the experience with mates held captive under the same conditions. Diaries have thus historically been produced by both individuals and groups, regardless of their degree of intimacy or their potential to appear in print. Since its very inception, the genre has been dialogic rather than monologic, hence obliterating the line between private and public.

Upon entering the digital era, the diary as a cultural (or literary) form appears to have survived in its many varieties and its layered complexity. Searching on the Internet today, one can find a plethora of digital forms, everything from travel blogs chronicling the climbing of Mount Everest to personal blogs commenting on books or music; from the spiritual journey of a born-again Christian to the intimate exchange of sexual experiences between teenagers, and from outbursts of psychological distress to the quotidian musings of a psychiatrist. If you search Google under 'blogs' you will find travel blogs, sex blogs, personal blogs, political blogs, religious blogs and what have you. The SlowTravelersBlog (www. 
slowtrav.com), including webcam pictures and essays mostly by hikers, could not be more different from the musings on Bitter-Girl.com. It is impossible to characterize some blogs as personal and others as public, and yet we typically characterize all of them as blogs. Obviously, privacy or publicness is not a standard feature of weblogs and it would be misleading to subdivide the genre accordingly.

The digital successor of the diary is as polymorphous as its paper precursor, and yet, when researching the new functions and forms of diary writing in the digital era, the old typology of the diary in terms of content and directionality still often informs the epistemology of the weblog. For instance, a 1998 Japanese study into the formal structures and uses of diaries on the Internet, departs from the notion that they can be classified according to their contents as 'records of fact' or 'expression of sentiment', or according to their directionality as 'written for oneself' or 'written for others' (Kawaura, Kawakami, and Yamashita 1998, 234-45). ${ }^{19}$ This classification along binary axes results in a new typology of diaries on the World Wide Web as 'memoirs', ‘journals', 'narrowly defined diaries' and 'open diaries.' The researchers' attenuated conclusion that writing a web diary is primarily communicative behavior, however, also applies to paper diaries. Digital cultural forms are often erroneously ascribed 'unique' features such as interactivity or communicability. As I argued above, though, paper diaries have always shown a peculiar mixture of intimacy and publicity, of individual and communal effort, of selfexpression and communication. If we look at weblogs, we can observe a similar blend. In order to explain the paradoxes and discontinuities embodied by diaries and blogs, we need to shift our focus away from genre typologies and pay more attention to how these forms are actually used.

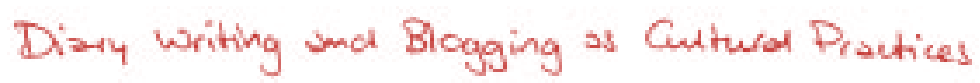

Many researchers, like the ones discussed above, assume that the digital diary is a seamless continuation of the paper diary, thus ignoring the emergence of a new mediating apparatus. The Internet, however, is not simply an amplification of the individual word processor, but is a new tool that also encompasses many features of the old. In our focus on technology, we often tend to underemphasize how social and cultural conditions change along with the apparatus. Both diary writing and blogging are interesting cultural practices - quotidian habits or daily rituals which gradually receive a place in a person's life. Cultural practices, in the past century, have become increasingly mediated: watching television, talking on the phone, taking pictures or writing e-mails are only a few of many potential communicative acts by means of which a person articulates herself. With the introduction of the Internet, some of these daily rituals are gradually changing, often fusing old practices with new conventions. For instance, e-mails can be regarded as 'remediations' of hand-written letters, but, more profoundly, the emergence of e-mail also substantially transformed one's daily ritual of communication and interaction, along with one's sense of physical or psychological presence - just as the telephone 
changed communicative patterns along with notions of proximity and presence a hundred years earlier. It is important to note that these changes always involve both technology and practice, the mutual shaping of which is firmly embedded in culture.

Writing a diary, of course, never happened in a social vacuum; the ritual occupied its own niche alongside other acts of communication, such as talking, listening, reading, etc. As a quotidian habit, diary keeping gives meaning and structure to someone's life. In the case of Anne Frank, writing a journal created a zone of silence and refuge in a small space, densely crowded and heavily trafficked by human interaction. Her daily ritual was an act of self-protection as much as self-expression. By carving out a discursive space, she was able to articulate her private thoughts and define her position in relation to others and the world at large. Diary writers fashion a habit by choosing a medium; the creation of that mediated habit is always inspired by cultural conventions and prevailing fashions. ${ }^{20}$ Quotidian acts such as diary writing should thus not only be regarded as stilled reflections of life, but as ways of constructing life. They always coexist amidst a number of other communicative habits and culturally determined practices.

For the contemporary blogger, the Internet is just one of a host of media through which to express agency, and blogging is one of many competing practices, such as speaking (both face-to-face and phone conversations), writing (letters, sms, e-mail), watching (television, film, photographs) and listening (music, talk). The practices that fill the mediated lives of today's youngsters are deployed concurrently and complementarily; the weblog offers a few amenities that other media lack, such as the ability to combine extensive written comments with pictures, tunes, links and clips, as well as the possibility to post something online to a large anonymous readership; blogging is potentially a multimedia practice - a combination of old-fashioned diary writing, letter writing, the exchange of cultural objects, publication, and even conversation. ${ }^{2 I} \mathrm{New}$ hybrid rituals always emerge in dialogue (and in competition) with already existing practices. Viewed from that angle, it should come as no surprise to find that, while about half of all personal blogs do, indeed, fully exploit the multimedia potential of the Internet, the other half contains no links at all. ${ }^{22}$ As the practice evolves, these numbers are likely to change, but at this moment of transformation we can observe how conventional habits of diary writing coexists with a truly multimedia version of blogging.

The networked computer is instrumental in the way a blogger simultaneously fashions his/her identity and creates a sense of community. Blogging both complements and interferes with everyday 'live' communication: weblog entries are part of a person's ecosystem of various community circles through which they move and shape their lives. Some of these circles overlap, some do not. The by and large reflexive nature of the weblog has its place within the contact zones of everyday life that each individual constructs, and which are usually a mixture of real-life and virtual experiences. Through their LiveJournals or Xangas, teenagers do not only express themselves, but create a communal sense of values and thoughts deemed worthy of being shared. In a weblog, one may blurt out confessions of loneliness and insecurity - behavior inhibited in face-to-face encounters - despite the fact 
that everyone in a peer group can potentially read these outbursts. Bloggers usually do not talk about what they say online, even though in real life they may speak to each other on a daily basis. ${ }^{23}$ Online posts can be read and responded to by immediate friends and relatives, while they may also invoke reciprocity from complete strangers, adding another dimension to the small world of immediate peers. The distribution features of blogging software are a subtler version of the lockand-key-diary; with each posting users can decide to whom they make content available - options ranging from 'just myself' to 'friends only' to 'anyone'. Defining one's readership is bound to define one's sense of inclusion in and exclusion from a community, whatever shape that community may take - actual or virtual, intellectually formative or emotionally supportive. In contrast to the paper diary, the weblog is part of a mediated continuum, a lived world in which the individual is always connected. ${ }^{24}$ Although reciprocity is the default mode of blogging, still half the number of internet diaries turn out to be non-reciprocal (Herring). Apparently, old habits of diary writing coexist with new connected practices, while they become gradually incorporated by a medium that shifts the technological condition from isolation to connection.

The inclusion and exclusion of (potential) readers from one's weblog constitutes an intricate game, the stakes of which are identity formation and community construction. Identity, as Australian media theorist Esther Milne claims, is always, in varying degrees, a performance: 'It is the result of complex cultural, technological, economic and institutional forces rather than being a natural, somatic or psychological process that is fundamentally independent of historical influences' (Milne 2004, 8). Current 'complex forces' are geared towards swift and easy distribution of ideas. In the past, the ability to expose oneself to a wider audience of unknown readers was something for which a paper diarist was previously dependent on a publisher who would print and distribute the diary, usually resulting in a considerable time lag between the moment of writing and of publication. A blogger can make her own decision concerning publication and distribution at the very moment of writing. Sharing intimate narratives with an anonymous readership is no longer a future possibility but an actual choice for webloggers; the effect of this technological option is immediacy - instant distribution, without intervention by a publishing institution. From a survey performed by the MIT Media Lab Sociable Media Group, we learn that $76 \%$ of bloggers do not limit their readership in any way, and they have no idea who their readers are, apart from a core audience (Viegas, 'Blog Survey').

Weblogs or digital diaries are perhaps primarily about synchronizing one's experience with others, about testing one's evaluations against the outside world. Blogging, aside from being an act of self-disclosure, is also a ritual of exchange: bloggers expect to be signaled and perhaps to be responded to. If not, why would they publish their musings on the Internet instead of letting them sit in their personal files? It may be instructive to compare blogs and blogging to the use of the mobile phone. In their study of teenager's use of mobiles, Alex Taylor and Richard Harper note how phone-mediated activities resemble established social practices such as gift-giving - the ritual of gift exchange is now extended to symbolic mes- 
sages (sms or spoken), and, like the material equivalent, rooted in a mental scheme of obligation and reciprocation (Taylor and Harper 2003, 267-296). Through a subtle system of shared norms for exchanging phones, rationing access to personal messages, and obligations to respond, users assign symbolic value to tangible or virtual objects. A similar process can be identified in blogging. Opening up one's secret diary to a selection of friends and relatives, and expecting them to do the same, is an old practice refurbished by webloggers. Attaching cultural items is quite similar to swapping music albums, books, or personal accessories - a system of sharing symbolic meanings with friends that is firmly rooted in the material culture of gift exchange. But the potential to open up this process to an anonymous and potentially large readership is new; bloggers are constantly connected to the world-at-large, and are aware of their exposure to it. Synchronization, however, does not prohibit self-reflection, just as privacy does not preclude openness. Old and new functions of diary writing thus peculiarly merge into a hybrid networked practice of blogging.

At first sight, a prime function of diary writing seems to be virtually absent in the practice of blogging: paper diaries were meant to fix experience in time, to freeze one's thoughts and ideas into words (and perhaps illustrated materials) to serve as a reminder of former experience later on in life. In contrast, blogging seems to be more about revising one's experience over time, allowing one to adjust one's former observations and reflections as time goes by and as personality evolves. This difference in function is all too easily ascribed to a material fixity of paper diaries as opposed to the evanescent quality of software or screen content. Yet if we focus on cultural practices in conjunction with technology or form, we may find this opposition to be quite ungrounded. For one thing, paper diaries were never 'finished' paper products; they were often exercises of writing prone to later revision, because of a changing insight, retroactive embarrassment, or due to a changing ambition or purpose in writing the journal. Anne Frank, for instance, started to write a revised version of her diary in March of 1944, several months before she was deported to a concentration camp. ${ }^{25}$ The two 'versions' of Anne Frank's diary signify how time changes a person's experience as well as her memory of that experience. Revising one's diary entries is inherent in personal growth, particularly at a younger age.

Weblogs obviously meet the revisionist need of a diary writer, as entries can be endlessly edited and deleted. Yet, from the contemporary weblogger's perspective, that does not obliterate the urge to fix experience. Blogging is often considered a transitory cultural practice, comparable to talking on the phone or sending short text messages. But even in the case of telephone conversations or short messages, the desire for storage and retrieval is evident. One of the teenagers included in Taylor and Harper's research admits that she would like to store each sms exchange on a memory card because she wants to recall her experience later: the message's physical properties (form, content, time, and date stamp) all work in combination to instill meaning in the physical (Taylor and Harper 2003). We can see a similar reconciliation of seemingly opposite functions in the use of digital diaries. For one thing, the very fact that bloggers use writing as a basic form of expression indicates 
a desire to secure these symbolic exchanges in some retrievable form. And even if weblogs look more like a written conversation or stream of consciousness, almost every software program contains an 'archive' holding selected entries and comments, going back to the very beginning of a person's weblog. Bloggers tend to value their archives, as their entries gradually turn into interesting memory objects of past experiences. In other words, the assumption that digital materiality inherently favors transience over permanence is hardly tenable in the face of the overwhelming popularity of weblog archives. Synchronizing experience and fixing experience in time are not at all contradictory functions, but they have perfectly merged in today's weblogs.

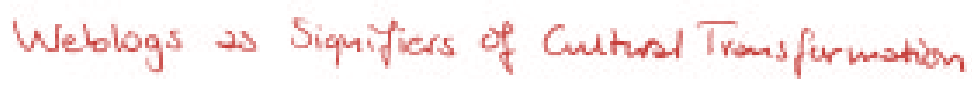

Looking at weblogs and the cultural forms and practices they engender, we can deduce an interesting reinvention of age-old rituals, newly attuned to the modalities of digitization. Like the writing of paper diaries, blogging is a process that helps express and order thoughts through rituals, thus defining a sense of self in relation to others. Diaries and weblogs are both acts and artifacts, in which materiality and technology are interdependent on their changing cultural form, their use and users. Rather than pinpoint differences and continuities, I have tried to signal how functions and features of the analogue and digital genre coexist and co-evolve. Some seemingly conflicting genre features that have always existed are now reconciled in the face of evolving hybrid practices, while other paradoxes persist. Even though pen and paper were gradually replaced by (networked) computers, multimedia materiality still reflects the personality and individuality that was formerly signified by handwriting and paper objects. The classification dilemma of distinguishing diaries as strictly private (written for oneself and by one person) or public (written to be read by others) does not disappear with the advent of weblogs, on the contrary, the ambiguity is amplified by the potential of instant publishing. And finally, the cultural practice of blogging easily blends the need to synchronize experience with the desire to fix and revise experience in time. However, analyzing the evolution of a single case of technology-form-practice has never been a goal in itself; rather, I would like to explain how this particular case signifies a larger techno-cultural transformation that is much more profound than its traces left on the World Wide Web. In tracking how a new hybrid practice of blogging evolves, it is crucial to acknowledge how it sustains old and constructs new epistemologies and how it indicates a transformation of important cultural notions, specifically the paired-off notions individual and collective, privacy and publicness, and memory and experience.

Individuality and collectivity are redefined in the face of a culture that values sharing. Weblog architecture favors a connected exploration of the personal; what the Internet does best is to create a forum for collective discourses. Although reciprocation is certainly not a condition for participating in the blogosphere, the default mode for diary writing has gradually moved from isolation to connection. Of 
all the weblogs present on the Internet today, some still resemble conventional paper diaries while others have morphed into completely new interactive formats, firmly rooted in Internet culture. Through weblogs, intimate reflections and revelations about personal, intellectual, and artistic preferences are consciously shared with both known and anonymous audiences. Weblogs and blogging might be seen as part of a larger participatory turn in culture. In this culture of sharing, the weblog finds its natural habitat so that the digital diary becomes instrumental as its multimedial modality equally allows for the creation of one's personal entries as well as for the exchange of cultural contents (clippings, files, songs). Blogging software and Internet hardware, in this argument, are neither neutral technical conduits nor simple commodities, but they are cultural artifacts facilitating a social process in which generalized exchange and participation are conditions for enacting citizenship.

However, there is another side to this techno-cultural transformation that often gets underemphasized. The culture of reciprocation is not solely based on linking the self to the Net, but also on linking the Net to the self. Tracing cultural or political preferences of other bloggers, one can decide to connect to people with similar tastes and preferences; it is precisely this feature that makes weblogs interesting for outsiders. With the use of fairly simple software applications like AllConsuming.net, it becomes increasingly easy to find a correlation between bloggers and the cultural products they mention via links or sidebars: books, music, television programs, movies, etc. ${ }^{26}$ Tracking software allows a glimpse of the patterns and trends that emerge out of the topics shared by a group. Coupled with vast databases like Amazon and Google, the possibilities for polling and marketing research are endless, explaining Google's eagerness to buy start-up companies like Blogger. ${ }^{27}$ Whereas many diaries (like OpenDiary and DearDiary) started out as small communities of like-minded individuals, many of these services are now owned by corporations. The downside of the culture of reciprocity is instant marketability: personal taste and cultural choices become instantaneously traceable and marketable to commercial ventures. In a networked environment, where information is constantly cached, weblogs have become gold mines for data diggers. For bloggers, social norms concerning individuality and collectivity appear to be in flux; old notions of personality and belonging persist, while the new media reality prompts a keener awareness of manipulative strategies enforcing individual taste and community building.

The same ambiguity applies to the blogger's notions of privacy and openness. As I pointed out above, privacy has always been an effect rather than an intrinsic feature of a paper diary's content, often achieved through one's familiarity with conventions for publication and publicity. Our norms and laws of privacy protection are still based on a strict distinction between ego documents and public records; if boundaries were often crossed in the past, for bloggers they become increasingly fuzzy. Emily Nussbaum notes in her journalistic-ethnographic report that bloggers have a 'degraded or relaxed sense of privacy', depending on your perspective: 'Their experiences may be personal, but there is no shame in sharing... [and they get back] a new kind of intimacy, a sense that they are known and lis- 
tened to' (Nussbaum 2004). There is not only no shame in sharing: bloggers take pride and find purpose in sharing. Privacy is an effect determined by a click of the mouse. Instant publication, however, changes the rules of the game. As the MIT Media Lab survey shows, bloggers are hardly concerned with the persistent nature of what they publish; the overwhelming majority publishes private information about themselves or other people without thinking about legal or moral consequences. Not surprisingly, more than one third of all bloggers have gotten into trouble because of things they have written in their blogs and the majority has little notion of defamation or liability when writing about others in networked environments. ${ }^{28}$ Their notions of privacy and publicness appear full of contradictions: comments are personal yet readable by everyone, intimate yet public. Old and new notions of privacy are contested in the blogosphere; courts and lawyers are currently wrestling with emerging questions like whether entries posted with restricted access can be 'stolen' when they are posted on an open website? Are public officials or state employees free to speak their minds in the 'private' sphere of restricted blog communities? It will take a number of years before this hybrid practice will have stabilized and become grounded in social and legal norms.

Weblogs do not only signal altering notions of individuality and privacy, but also of personal memory in relation to lived experience. The paper diary reflected the idea that the memory object is a petrified, unchangeable relic, stored in its authentic form and retrieved to invoke a past experience. When a diary's contents were still published by means of an intermediate process of editing, printing and distribution, we were mostly concerned with how the 'original' words - assumedly the recordings of experiences - matched the words published in print. The fusion of old and new technologies results in a hybrid tool that seamlessly combines communicative and archival functions; blogging allows for exchanging, storing, and revising entries all at the same time. ${ }^{29}$ Blogging itself becomes a (real life) experience, a construction of self that is always mediated by tools for communication and expression; in other words, the medium is the experience, not the message. If the meaning of experience is slowly changing, so is the meaning of memory. As time proceeds, memories of experiences inevitably evolve, revising one's past inscriptions is a natural part of a process of personal growth. Rather than being fixed in material paper objects, memory mutates by means of digital materiality. Although the Internet is often characterized as a transient, evanescent medium, weblogs have both the ability to fix and the potential to morph; blogging constitutes a new concept of memory, allowing for preservation and erasure simultaneously.

Bloggers are retooling the practice of diary writing, meanwhile creating a new type of cultural knowledge and social interaction via their tools. The reciprocity inherent in networked systems points at a profound reorganization in social consciousness. Media change may be traced through its technology-materiality, as Kitzman proposes, or through its specific cultural forms, as Bolter and Grusin suggest, but as I have tried to show here, it is important to examine technological and cultural changes in constant connection with socio-cultural practices, in order to come to understand larger socio-cultural transformations. In the case of weblogs, I have argued how old and new technologies, forms and practices co-exist and yet 
co-evolve into hybrid practices. These hybrid practices both reflect and construct new social norms and cultural concepts, such as individual and community, privacy and publicness, experience and memory. In a period of transition, these concepts fluctuate and will continue to fluctuate, but unraveling complex transformation may help us sort out newly emerging cultural values. If we look back at the cartoon, cited at the beginning of this article, we now comprehend that the woman who starts typing her weblog just after denying her partner's question whether she keeps a diary, is not necessarily lying. In fact, the three frames of this cartoon perfectly reflect the ambiguous reality in which millions of bloggers find themselves today.

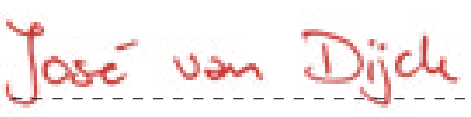

SIGN HERE! 
Notes

1. I would like to thank Eric Ketelaar for bringing this cartoon to my attention and for his constructive comments on earlier drafts of this paper. I would also like to thank Sonja Neef for her help in sharpening my thoughts.

2. Emily Nussbaum, in 'My So-Called Blog', New York Times, January 11, 2004, Magazine (digital version), bases these figures on an October 2003 inquiry by the Perseus Development Corporation, a company that designs software for online surveys.

3. Kitzmann emphasizes that the concept of 'remediation' implies too much linearity and hierarchy, proposing instead to study media change in the context of the much wider phenomenon of 'material complexification', in which change is not cumulative 'but [measured by] structural shifts that may lead to growth, contraction, stasis, or a combination of all three' (51).

4. On the importance of materiality (both paper and digital materiality), see N. Katherine Hayles, Writing Machines. Cambridge: MIT Press, 2002.

5. See also Sonja Neef, 'Authentic Events. The Diaries of Anne Frank and the Alleged Diaries of Adolf Hitler', elsewhere in this volume.

6. Jane Zhang, archivist at the University of Calgary, writes: 'Handwriting is a physical as well as mental activity, directly influenced by any change that takes place in the human body and mind. Therefore, an individual's handwriting is habitually viewed as his own personal mark, which distinguishes him not only from others, but also from his own past and future' (p. 43). Jane Zhang, 'The Lingering of Handwritten Records' in: Proceedings of I-Chora Conference, 38-45, University of Toronto, October 2-4, 2003.

7. Neef studies the 'Verkörperung' (embodiment) of handwriting in relation to the 'Entkörperung' (disembodiment) of computer-mediated writing.

8. See also Eric Ketelaar, 'Writing Archival Machines', elsewhere in this volume.

9. I concur with Belinda Barnet, who argues that we should consider both text and materiality and both representation and technology, as vital and constitutive components in the construction of written memory. See Belinda Barnet, 'The Erasure of Technology in Cultural Critique', in: Fibreculture 1: 1 (2004): 6.

10. Friedrich Kittler (1999), states: ‘Only two things were lost during this mechanization of writing: first, the intimacy of handwritten expression, which nobody is willing to relinquish voluntarily, particularly in personal correspondence; and second, a centerpiece of occidental symbolic systems...' (186). And later in this chapter: 'The typewriter tears writing from the essential realm of the hand, i.e., the realm of the word' (198). This idea does not originally stem from Kittler, but Kittler is referring to Heidegger's Parmenides lecture.

11. Susan Herring, sociologist specializing in computer-mediated communication, quotes in her paper 'Weblog as Genre, Weblog as Sociability' the number of 4.12 million users from the statistics of the Perseus group by October 2003 . This number of bloggers, which is growing exponentially, includes hosted weblog services; $34 \%$ of these logs are used actively (see: <http://ella.slis.indiana.edu/ $\sim$ herring/ssc.ppt $>$ ). Herring uses the following operational definition of a weblog: 'a frequently modified webpage (and associated pages) containing individual entries typically displayed in reversed chronological sequence.'

12. For a short introduction to weblogs, see 'The History of Weblogs' available at: $<$ http://newhome.weblogs.com/historyOfWeblogs > (last checked on March 16, 2004). The personal homepage, which became popular between 1997 and 2000, is generally regarded as the precursor of the weblog. For an interesting sociological analysis of the personal homepage, see Charles Cheung, 'A Home on the Web: Presentations of Self on Personal Homepages', in: David Gauntlett (ed.), Web.Studies: Rewiring Media Studies for the Digital Age, 43-51. London: Arnold, 2000.

13. There are few informative books on weblogs and blogging. One of the few collections of articles on the initiative of weblogging is John Rodzvilla (ed.), We've Got Blog: How Weblogs are Changing Our Culture. Cambridge, MA: Perseus Publishers, 2002.

14. Emily Nussbaum, citing Perseus numbers, notes that $90 \%$ percent of bloggers are between 13 and 29 years old, meaning teenagers and young adults. Susan Herring found the producers of weblogs she included in her sample to be equally divided between male and female, teens and (young) adults. 
15. Susan Herring defines the 'blogosphere' as 'the universe of blogs available (mostly publicly) on the World Wide Web'. 16. Thomas Mallon, author of $A$ Book of One's Own: People and their Diaries (New York: Ticknor and Fields 1984), a standard work in this area, distinguishes at least seven types of diaries, labels he attributes to the profession or characterization of their authors ('chroniclers' 'travelers' 'creators', 'confessors', etc).

17. Philip Lejeune (1993), inventorizes various types of autobiographical writing (diary, letters, autobiography) by their 'morphological' features.

18. Eric Marty $(1985,87)$ argues that the old-fashioned paper diary was always communicative in purpose. He labels the diary's imagined addressee 'l'Autrui' to distinguish him from 'l'autre' or a real, existing person. Susan Herring defines genre as 'a distinctive type of communicative action, characterized by a socially recognized communicative purpose and common aspects of form'.

19. In defense of the authors of the Japanese study of online diaries, suffice it to note the year in which their article was published, 1998, which marked the early beginning of an explosion of online blogging, a development no one could have predicted at that time.

20. As David Chaney, in Cultural Change and Everyday Life (Houndmills: Palgrave, 2002) observes, everyday life is a creative project 'because although it has the predictability of mundane expectations, it is simultaneously being worked at both in the doing and in retrospective reconsideration' (52).

21. 'As mediated human communication becomes more and more non-linear, decentralized, and rooted in multimedia, the distinction between orality and literacy becomes less evident and less important' argues Jan Fernback in 'Legends on the Net: An Examination of Computer-mediated Communication as a Locus of Oral Culture', in: New Media and Society 5: 1, 29-45, 2003. Citation on page 29.

22. Herring concludes from a content analysis of 357 personal blogs that the blogosphere is densely interconnected via links in the sidebars of blogs. However, she still found that more than half of all blog-en- tries contain no links and that most blog entries receive no comments, confirming my assumption that the weblog is a hybrid of the old-fashioned paper diary and the new networked form.

23. Emily Nussbaum, in 'My So-Called Blog' cites a number of teenagers actively engaged in blogging.

24. As British sociologist John B. Tompson has eloquently argued in The Media and Modernity: A Social Theory of the Media (Cambridge: Polity Press, 1995): 'individuals increasingly draw on mediated experience to inform and refashion the project of self. Mediated rituals enable "intimacy at a distance"; for the generation living in the digital age, the continuous switching between real live and mediated communication is quite normal' (233).

25. Inspired by the Dutch Minister of Education, G. Bolkstein, who, on March 28, 1944 urged citizens to keep and save diaries from the time of occupation for publication, Anne Frank decided to revise her diary and turn it into a book. As we can see from this example, the future possibility of a large unknown readership prompted Anne Frank to make revisions, as much as did new insights and personal growth.

26. On the function and technology of AllConsuming.com, see Erik Benson, 'All Consuming Web Services', <http://www. xml.com.lpt/a/ws/2003/05/27/allconsuming.html >. (Last checked October 4 , 2004). Benson favors this kind of weblog tracking over previous method like Nielsen ratings or random polls; the attraction of weblogs is that 'the data is out there, the data is free, and the data is extremely interesting'.

27. Pyra Labs, one of the first start-up companies who designed Bloggersoftware, was purchased by Google in 2003. See Leander Kahney, 'Why Did Google Want Blogger?', Wired News, February 22, 2003, see: <http://www.wired.com/news/ technology $>$ (last checked 4, May 2004).

28. For a website with survey findings, see Viegas.

29. According to the MIT survey (see Viegas), almost $75 \%$ of all bloggers indeed edit their past entries, which varies from punctuation and grammar to contents and names. 
Works Cited

Bolter, Jay and Richard Grusin. Remediation: Understanding New Media. Cambridge: MIT Press, 1999.

Decker, William Merrill. Epistolary Practices: Letter Writing in America before Telecommunications. Raleigh: University of North Carolina Press, 1998.

Derrida, Jacques. Archive Fever: A Freudian Impression. Chicago: University of Chicago Press, 1995.

Didier, Beatrice. Le Journal Intime. Paris: Editions du Seuil, 1976.

Herring, Susan. 'Weblog as Genre, Weblog as Sociability', available at: <http://ella.slis. indiana.edu/ herring/ssc.ppt>.

Kawaura, Yasuyuki, Yoshiro Kawakami, and Kiyomi Yamashita. 'Keeping a Diary in Cyberspace', in: Japanese Psychological Research 40: 4, 1998.

Kittler, Friedrich. Film, Gramophone, Typewriter. Stanford: Stanford University Press, 1999.

Kitzmann, Andreas. 'That Different Place: Documenting the Self within Online Environments' in Biography 26.1 (Winter 2003): 48-65.

Lejeune, Philip. Le pacte autobiographique. Paris: Editions du Seuil, 1993.

Mallon, Thomas. A Book of One's Own: People and their Diaries. New York: Ticknor and Fields, 1984.

Marty, Eric. L'ecriture du jour. Le journal d'André Gide. Paris: Editions du Seuil, 1985.

Milne, Esther. 'Email and Epistolary Technologies: Presence, Intimacy, Disembodiment', in: Fibreculture 2, 8, 2004.

Neef, Sonja. 'Die (rechte) Schrift und die (linke) Hand', in: Kodikas/Ars Semiotica 25: 1, 159-176, 2002.

Nussbaum, Emily. 'My So-Called Blog', in: New York Times, January 11, 2004, Magazine (digital version).

Ong, Walter. Orality and Literacy: The Technologizing of the Word. London: Routledge, 1982.

Piggott, Michael. 'Towards a History of the Australian Diary', in: Proceedings of I-Chora Conference, University of Toronto, October 2-4, 2003.

Taylor, Alex S. and Richard Harper. 'The Gift of the Gab? A Design-oriented Sociology of Young People's Use of Mobiles', in: Journal of Computer Supported Cooperative Work 12: 3, 267-296, 2003.

Viegas, Fernanda. 'Blog Survey: Expectations of Privacy and Accountability', available on <http://web.media.mit.edu/ fviegas/survey/blog/results.htm>.

Yakel, Elizabeth. 'Reading, Reporting, and Remembering: A Case Study of the Maryknoll Sisters Diaries', in: Proceedings of I-Chora Conference, University of Toronto, 142-150, October 2-4, 2003. 


\section{Haxk on the Document: Anold Dreybldt's T Archive}

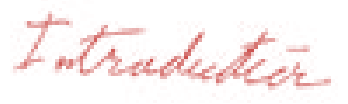

I have been working for many years on the development of an artistic practice that has involved the acquisition, administration and display of historical archival source materials. The 'T Project' is one of a series of ongoing works that looks at the archival traces of the individual as represented in written storage.

Whereas parallel projects, such as my Who's Who in Central \& East Europe 1933 (Dreyblatt, 1991, 1995) are based on biographical data from thousands of people as a representation of the collective, the 'T Project' concerns one individual, a marginal and mostly forgotten Central European historical figure whose multiple identities span three continents (Europe, North America, and Asia) and obliquely touch on many of the most important events of the pre-war period. More importantly, during the last 28 years of his adult life, Mr. T was followed and observed nearly every day by the intelligence services of various world powers.

This enormous effort in collecting documents resulted in the accumulation of thousands of daily reports and various forms of correspondence between 1915 and 1943, forming a vast communication network in which the observation of the activities of one individual becomes a kind of international discussion held over three decades between intelligence agencies, which is, in turn, cross-referenced in my artistic projects with historical events, international personalities, and geographic locations.

In this essay, we will examine the roles of handwriting in this archive of documents about $\mathrm{T}$, and interrogate the ways in which handwriting relates to other material dimensions of the documents. We will write in two 'voices': I, Arnold Dreyblatt, speak in the first person about my work, while Jeffrey Wallen comments on it in the indented paragraphs. 


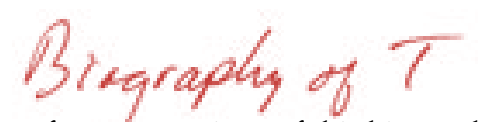

One may look at a series of representations of this biography, which in turn trace the development of this project. Here is a selected list of chronological events, as they would appear in a short biography of a Mr. T:

- born April 4, 1879, Paks, Hungary

- student at Budapest Drama Academy

- conversion to Christianity

- adopts 'name' Lincoln

- Protestant missionary in Montreal

- social research in Belgium and France

- election as member of Parliament in England

- oil speculation in Romania and Galicia

- fraudulent business deals

- under arrest in Bucharest

- arrested in Brooklyn as German spy

- escapes from prison

- rearrested

- deported to England

- imprisoned in England as German spy

- suffers nervous breakdown

- involvement with German rightists

- involvement with Kapp Putsch in Berlin

- encounters Adolf Hitler

- involvement with the White International

- arrested and tried in Vienna

- arrives in Shanghai

- involvement with rebel Chinese warlords

- obsession with Tibet

- abandons sons in China

- ruined in Monte Carlo

- mystical delusions in Ceylon

- second son sentenced to death for murder

- adopts name 'Chao Kung'

- Buddhist missionary in Europe

- heads Buddhist monastery

- letters to Adolf Hitler

- visits Canada

- deported from Liverpool

- establishes 'League of Truth'

- seeks return to Hungary

- calls for world peace

- espionage in Japanese-occupied China

- contacts with Nazis

- dies October 6, 1943, in Shanghai 
While the form is familiar, the seemingly linear format betrays an unstable pattern, jumping geographically and professionally in a refusal to be 'pinned-down' to one historical identity. In fact, Mr. T was not only observed continuously by the intelligence services, he, in turn, furnished a stream of misinformation, countless false identities or aliases such as: Abbot Chao-Kung, Theodor Lakatos, Heinrich Lamprecht, Thomas Langford, Dr. Tibor Lehotsky, Jack Fisher, I.T.T. Lincoln, Thomas Langford, Thomas Lorinz, Vilmos Ludwig, Wilhelm Ludwig, H. Ruh, Henry Fischer, Thomas Tandler, Theodore Trautwein, Dr. Johann Lange, Patrick Keelan, Joseph Schlesinger.

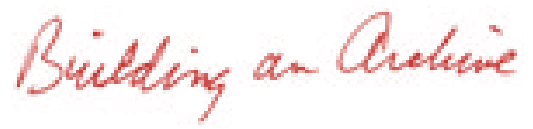

For this work, I created a duplicate or mirror archive, which entailed the acquisition of copies of over 4,000 intelligence documents from State Archives in Europe and North America. ${ }^{I}$ It was through this long process of data acquisition, and then the administration of these data materials, that the focus of the project moved away from and beyond the familiar chronology or biography of T. I became fascinated with issues of fragmentation and loss and with the dynamics of the storage and migration of data through governmental agencies and archival institutions. Within the transformations of the artistic project, these 'originally sanctioned archival copies' were re-scanned, digitized, altered, displayed as paper and projected, re-archived and re-lent.

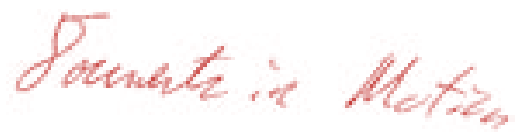

The 'T Project' follows the physical movement of paper as an 'original' passes from hand to hand, being sent and received internally within an agency and externally from agency to agency, often around the globe, by post and by telegraphic cables. Carbon copies are made and forwarded, and in the process are annotated, signed, stamped and initialed, leaving a 'paper trace' or evidence revealing a network or 'biography' of the document itself. Eventually the document becomes inactive, is filed away, migrating in medium from microfilm to photocopy and finally to digital bits and bytes.

Recently, a major news story in the us revealed that the laboratory director of the Secret Service had lied under oath in his testimony during the Martha Stewart trial (Glater, 2004). Stewart was perhaps one of the most powerful and richest businesswoman in the us, and she was on trial for having lied about her reasons for selling her shares in another company after having received inside information that that company's share price was about to collapse. A $M r$. Stewart, the Secret Service agent (and no relation to Ms. Stewart), had testified about the results of his analysis of a handwritten 
annotation to a document in the files of Martha Stewart's stockbroker. Mr. Stewart testified that his analysis of the notation '@60' showed that it was written in an ink different from 'other marks on the documents', and that it was therefore likely that this notation had been added separately, and at a later date, from other handwritten entries on the page. The defense countered with their own ink expert, who testified that the analysis of the ink did not support any of these conclusions.

At stake in the testimony was whether the analysis of certain handwritten annotations to a document can reveal the intention of another party (the intentions of Martha Stewart, and her stockbroker who made the annotations supposedly in response to her orders). Did she, in fact, intend for these shares to be sold automatically if the price ever fell to $\$ 60$ a share? And can the point in time at which this communication of an intention took place be determined? The handwritten mark, from the perspective of both the prosecution and the defense, is privileged, as providing access to an understanding, an agreement, that stood only on the margins of, as an addendum to, the more formal set of transactions, which either confirmed an order communicated verbally over the telephone, or, more sinisterly, from the prosecution's perspective, was intended to give the false appearance of the prior existence of such an agreement. For the prosecution, this handwritten annotation was an illegal, after-the-fact alteration - a falsification of the document.

Ironically, a couple of months after the trial, the Secret Service expert on the authenticity of documents (he had also served as an expert on 'the authenticity of documents used in a Nazi war crimes case' - such comments in newspaper articles are always meant to show us that the person has had direct contact with the most overpowering event of the 20th century) was now being accused of lying about his personal role in the analysis of the ink, and about the point in time at which he gained knowledge of the fact that two of his subordinates were writing a book containing a chapter on 'a certain type of ink analysis'. During the trial he claimed he had performed the ink tests while analyzing the document, whereas now it was revealed that he had not performed any of the tests and had not even participated in the analysis at all (the work had been done entirely by his subordinates); and that, at the time he appeared on the witness stand, he had no knowledge of the book his employees were writing. The authority of the handwriting and document expert is based on the presumption that he can scientifically demonstrate whether someone had been present at a certain point in time (whether the document reveals their presence) and that he can reveal to us facts about the temporal chain of events in the life of a document (whether it was produced before or after a certain time; certain 
marks were simultaneous to or later interpolations on the earlier content). Now the expert was being prosecuted for these identical issues: for lying about having a direct, 'hands on' relation to these documents, and for having a knowledge of the intentions of others at an earlier time than he actually had.

I think this incident, which was 'newsworthy' only because of the celebrity of Martha Stewart, serves as an allegory for much of our current relation to texts and to handwriting. In our era of word processing, telecommunications, and the Internet, handwriting is now likely to be something added later, a note on another text, rather than the mark of the original, the trace of the earliest intention, the first stage in the life of a document. The handwritten is now largely the mark of a reader; it calls attention to the hands through which documents have passed. The 'analysis' of handwriting, rather than revealing to us an individual identity, an original intention, and a specific moment of presence (the moment of the coincidence between the writer's thinking and the written expression of that thought), will instead tell us more about the many different ways in which the hand interacts with the written.

Yet we are also still driven by the desire to establish the priority of the human agent in relation to the transmission, circulation, reproduction, and migration across media of writing. The fear of the reversal of perspective, in which the human becomes merely the servant of the written, is an old one, and is the subject of powerful stories by Melville, Kafka, and many others. 1 In the age of the video replay, the simulation, and the delayed 'live' transmission, we especially want to be able to come into contact with or at least determine the presence of the author, and to be able to fix exactly the point in time in which a written event takes place. The Stewart trials reveal yet again that the bureaucratic institutions charged with preserving the belief that we can read, can retrace, and can fix the precise meanings of the marks left by our hands are themselves thoroughly haunted by the processes of circulation and remediation that undermine any claims to clear and direct knowledge about the presence, moment of action, and intention of an 'individual identity'.

Arnold Dreyblatt's work, and his T Projects in particular, help us reflect on and think through these dynamics. These projects revolve around the remediation and redisplay of a huge collection of documents, gathered from various archives, regarding the life of a marginal world-historical figure (this oxymoron, or tension between 'marginal' and 'world historical', will play out in many ways). Through different media, they present to us or give us access to the archival traces of an individual biography, and help us to re-orient our perspective and to understand more clearly important aspects of our relation to documents, to handwriting, and to remediation. 
Dreyblatt's work is not simply an aid to critical understanding, or a challenge or resistance to the conventional ways of thinking. These projects do much more than mark the shift in perspective from the 'buman' to the 'bureaucratic', from a biographical life and from the generation of a document to the archive, to the transmission and circulation of texts, and to the life and afterlife of documents. Rather, they immerse us in, and encourage us to explore, reflect, and play with, the layers and the traces of the passage of a person through the observation, transmission, and information storage systems of the major world powers.

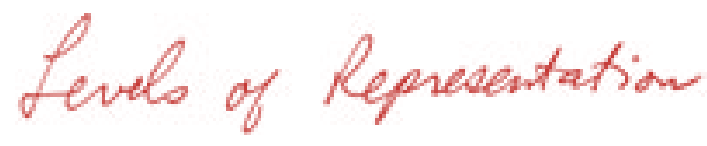

We can move to a second level of representation, in which the chronological list of biographical events is transformed into an administrative, bureaucratic structure that reflects a categorization and systematization performed by the archival institutions upon the documents:

\section{Record Group 59}

11.41T73

Goldstein to Roundtree, 10-11 July, 1914

Record Group 58-137, Book 1, Page 105-70, 4 October 1946

Record Group 65

FBI OG 500\BS202600-1356

State Department Decimal File

862.2.898

Court Extradition Hearing and Related Documents

Records of the Office of the Special Agent

The above represents a frozen moment in the archival process in which the chronological biographical or autobiographical form is fragmented and exploded into thousands of individual files, found under various classification systems and in diverse geographical locations. The identity and credit card databases, which reduce the contemporary individual to a number, find their origins in this administration of archival data.

At a primary level, the T-projects have as their basis the reproduction of several thousand pages of documents concerning ' $T$ ', and the remediation and redisplay of selections from these documents. This is done through newly created 'original' versions of these documents; or through the creation (as one part of a larger 'Memory Arena' project) of an actual working archive (staffed by archivists) 
where one could search, request, and then read a version of some of these documents; or, later, through various computer interfaces. In order to make this less abstract, I will discuss a couple of documents in order to reveal some of the ways that Dreyblatt's projects shift our processes of reading and the processing of this material (selecting and displaying one document in this manner, however, short-circuits the aesthetic mediation and the recontextualization that is integral to the project).

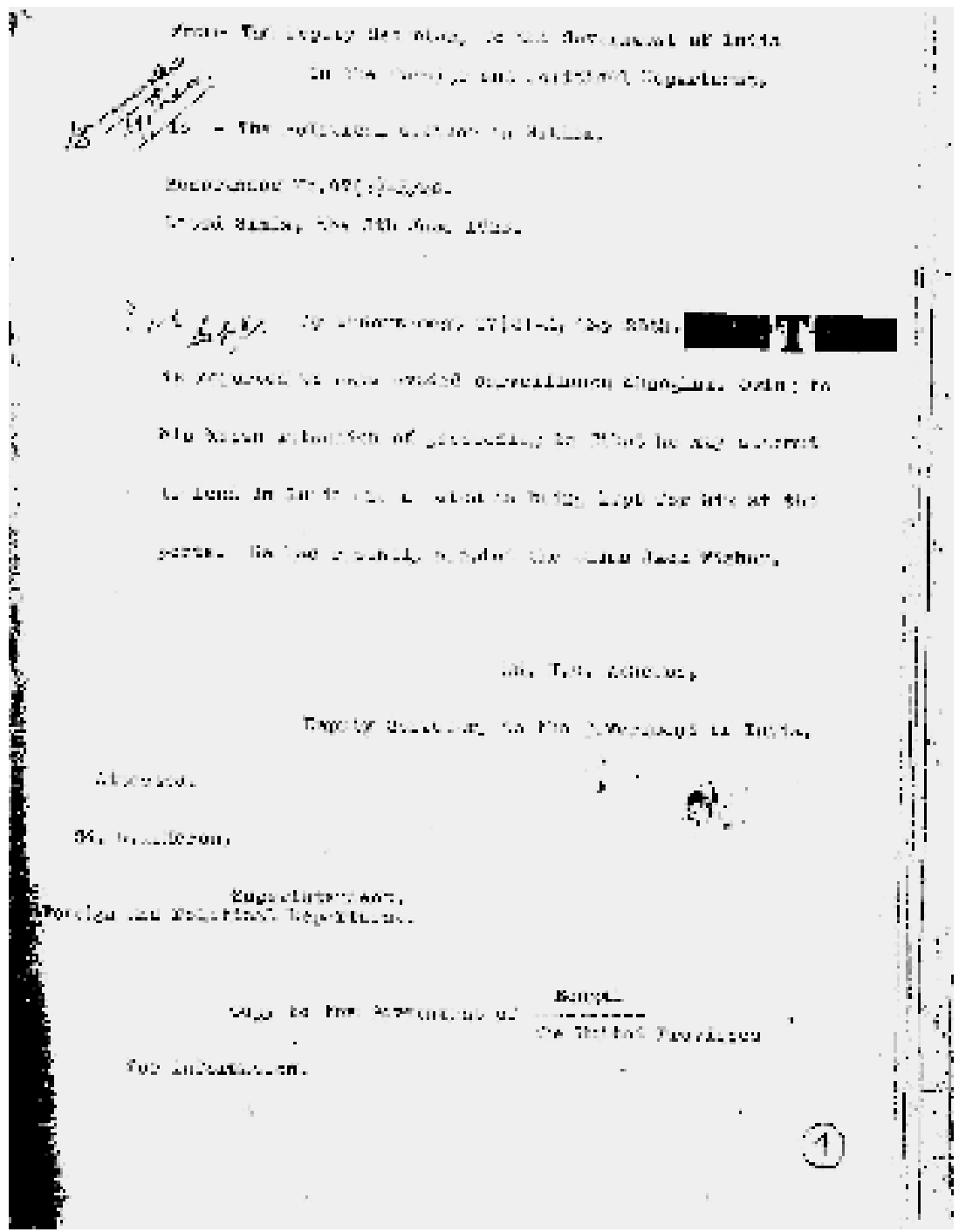




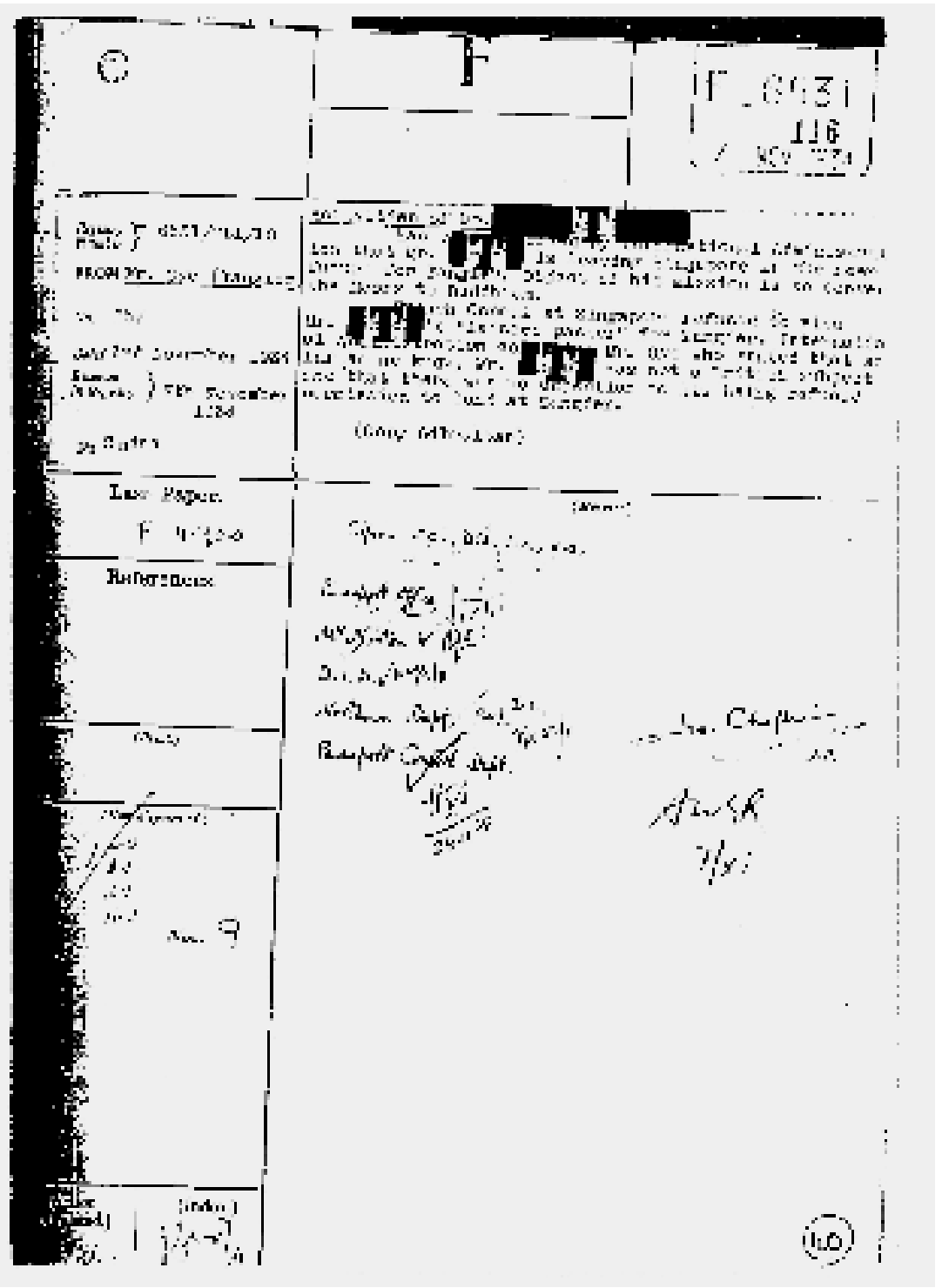

2. Document \#100

What leaps out at once is the immensity of the document-producing structures - Deputy Secretary, Government of India, Foreign and Political Departments, Superintendent, Government of Bengal, and so forth-rather than the figure who is the apparent focal point. Dreyblatt has stamped out the name of the figure with '-T-', adding another layer to this palimpsestic document, both defacing and drawing attention to the apparent connection thread of these papers (though here, the recently adopted alias 'Jack Fischer' remains unmarked). More powerful still are the 'marginal' handwritten notations: 'Communicated by India' - who wrote that? Its literal 
meaning (with the synecdoche here of the whole standing in for the part, 'India' standing for one officer and one office within the imperial governing structure of India) seems clear, but what sort of performative speech act is it to note on this document, 'Communicated by India'? And what follows the content of the message is equally intriguing: a name and title; then 'Attested' with another name and title; and especially the 'copy to' with its wonderful fill-in-the-blank quality of another imperial province (not a person or office), here 'Bengal'.

The scope and craziness of this 'mission' is mind boggling, but there is also a euphoria (and a vertigo) that opens up on the surface of the document itself. The 'content' here occupies only a small rectangular box. The other compartments ('registry number', 'from', 'received in registry', 'minutes,' 'References', 'Last paper', ' how disposed of)'; the stamps acknowledging receipt and processing of the document; and the various hand-written annotations under 'minutes' and 'how disposed of' (marking the different offices to which the document has been sent, and/or the offices and people who have reviewed the document?) draw our attention and our imagination inexorably to all the circuits of transmission, review, and reproduction through which information is being passed, and to our own relations to and implication in these circuits through this further 'remediation'.

I want to make a few fairly straightforward points about some of the shifts in perspective that Dreyblatt's work call forth. A basic transcription of these documents, focusing on their 'content' and on simply following the target ('-T-'), would filter out many of the layers and traces of circulation in order to sharpen the picture of the story of an individual, and his passages through 'world history'. Dreyblatt's 'remediation' gives us a different standpoint: the 'life' of the document. Rather than drawing us toward trying to ascertain or understand a precipitating event, the true happening, some actual moment in the 'life' of a person that generates these series of observations (what was $T$ really trying to accomplish in November of 1934?), this redisplay of material displaces our attention from an origin to an afterlife, and from the individual to the archival, to the now public traces that continue to circulate. Any desire to recover an original moment of intention or of action or of observation or of inscription or of transmission (and the multiplication of possible starting points already testifies to a crisis of determination) gives way, when one becomes drawn in to the 'T Project', to other fascinations. By reorganizing, cutting up, reconnecting, and/or redisplaying the material, Dreyblatt helps make visible to us the rever- 
berations - the further movement, circulation, and connection - of each point of contact between an individual and state networks of power.

A passage from Jacques Derrida's 'Plato's Pharmacy' provides an interesting perspective on Dreyblatt's use of the archive:

The 'outside' does not begin at the point where what we now call the psychic and the physical meet, but at the point where the mnemè, instead of being present to itself in its life as a movement of truth, is supplanted by the archive, evicted by a sign of re-memoration or of com-memoration. The space of writing, space as writing, is opened up in the violent movement of this surrogation, in the difference between mnēmē [living, knowing memory] and hypomnēsis [re-memoration, recollection, consignation]. The outside is already within the work of memory. (p. 109)

Derrida argues that Plato's hope for a 'mnēme- with no hypomnēsis, no pharmakon' is an impossible dream, and that the 'living, knowing memory' is always already being 'supplanted by the archive'. Dreyblatt's work shifts our perspective, so that the categories of 'inside' and 'outside', 'living' and 'recollected' (and one might add 'handwritten' and 'machine printed'), lose their ground in the 'simple alternative presencelabsence'. The archive itself is for us no longer 'hypo-,' no longer 'beneath', 'under', 'in a lower relation'.

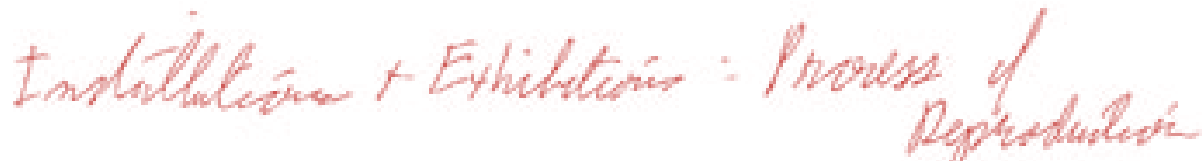

I will discuss three differing instances involving this mirror $\mathrm{T}$ archive in my artistic practice over the last twelve years.

The installation T-Docs (1993) playfully contrasts the nature and authority of the 'original' as found in the archival institution with the display of the original in art. In T-Docs, 110 original archive documents (officially sanctioned institutional copies) have been digitized and faked by specially developed printing techniques utilizing diluted inks and applied to the reverse side of postwar East German archival material, which poses questions about the identity of both the subject's personality and the authenticity of the documents themselves. The documents are presented in chronological order as both archival and 'art' originals (which are ironically not signed by the artist). All occurrences of 'Mr. T's' name are digitally removed and hand-stamped with a large red ' $T$ '. The reprinting process attempts to retain the 'hand', in this case, as the 'typing hand' along with subsequent handwritten commentary and markings which later appeared, including this 'real' hand stamp. 
Exhibitions of this work often lead to viewer irritation because they raise the question: were these illegally displayed 'real' documents actually stolen from an archive? Yet the biographical content is too improbable to be 'real' and therefore 'original'. Are the documents faked or is the content 'original' in terms of being 'thought up' and thereby 'faked'? The art world is immune to these problems, being unconcerned with 'real' but rather with the 'original hand' of the artist, and with the artist's 'originality'.

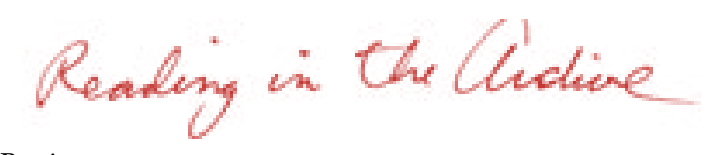

\section{The Reading Projects}

In a second instance, the document is no longer isolated and displayed on a wall but is now found in its rightful home in a file folder in an archive box, within a filing system and inside a 'real' archive. Here the 'original' and its copies are stored, protected, categorized, according to archival practice.

Influenced by my experiences in document collection at various archives for the 'T Project', I began a series of projects in which I became interested in simulating the living environment in which data is stored with the intention of making this process transparent and interactive within an installation and performance context. All of the documents contained in the archive could be searched for in a cata$\log$ (card or digital) and ordered and lent out to be read in a special reading room. Copies of the ' $\mathrm{T}$ ' documents were among the archive holdings.

re: Card Catalog

The Archive contains the following types of Files:

1. Class 'D' Files: copies of all Files that are read in the Arena.

2. Class 'QI, QII' Files: Personal Biography Data Files of Visitors and Readers and from Memory Arena I and II.

3. Class 'QIII' Files: Personal Biography Data Files for Visitors and Readers from Memory Arena III; (entered into the Archive as they are filled in).

4. Additional Archive Material including: texts by Archivists (Class 'A' Files); copies of Original 'T' Files (Class 'T' Files), etc.

Sections 2 through to 4 of the archive are available for reading by visitors for a limited time at tables within the Reading Room Area. The archive personnel assist visitors in the use of the card catalog and in the selection and ordering of files.

The viewer is now a participant in a temporary functioning archival system. One cannot browse the 'stacks', one must know what one is looking for beforehand. One is only allowed to browse the catalog, where one must imagine 'content' from the bureaucratic classification of numbers, dates, and short titles (which may be designed to protect rather than to reveal!). One must wait for a functionary to locate and deliver the file. One has a period of time in which to hold the 'original' in one's hands before returning it to its home in the 'archive', and one must of 
course sign out the document with one's signature. The participant, filling out a request form for documents by hand, and then reading them, re-enacts the role of those in the various agencies who had earlier reviewed these same documents. The reader handles the documents, and leaves behind the traces of his or her own pathway through the archive- the handwritten requests and signatures for documents. In addition, participants in the readings and in the archive were invited to fill out biographical questionnaires and to donate them to the archive's administration, thereby taking part in a process of autobiographical, bureaucratic description.
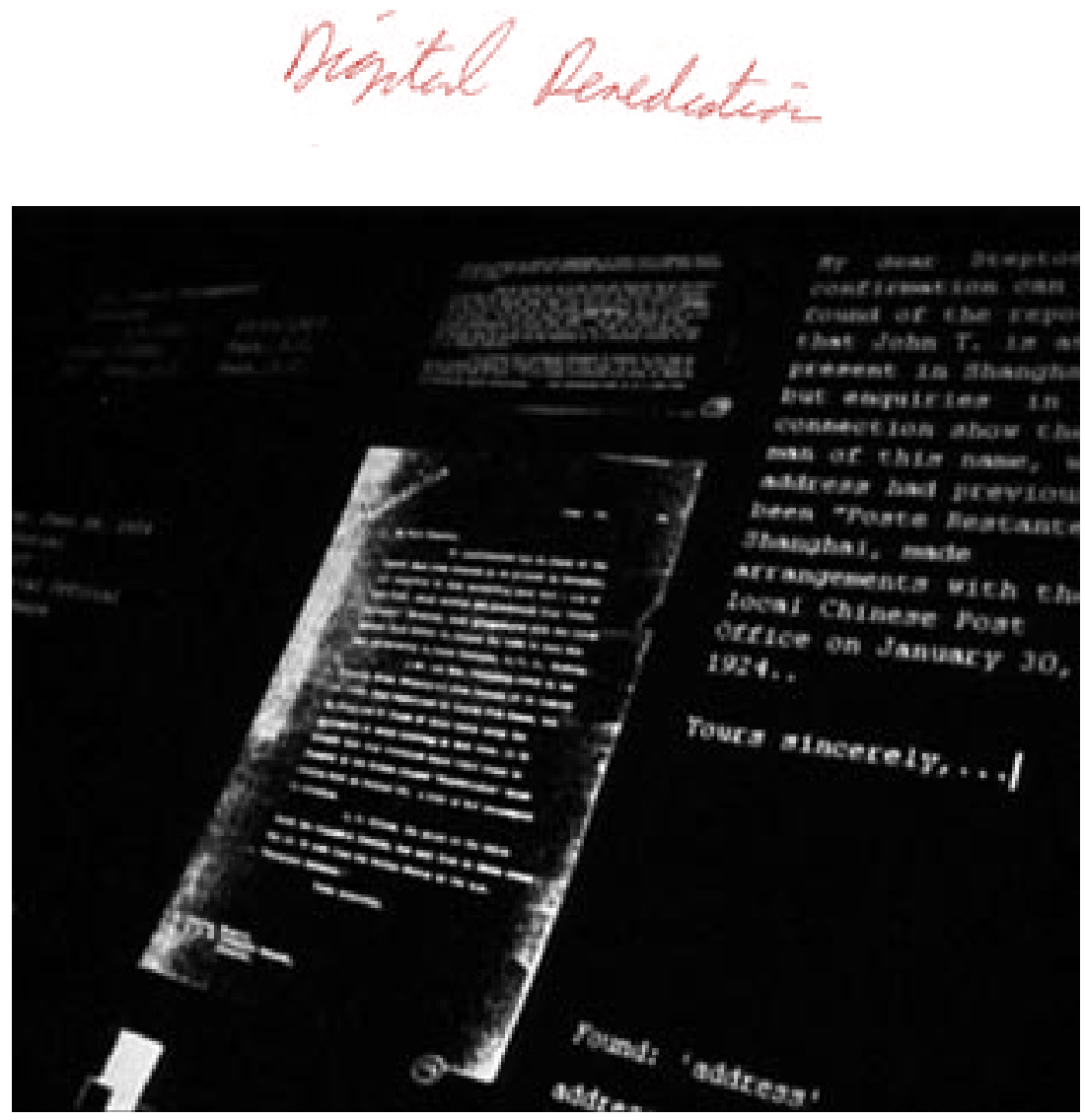

3. T-Mail

In a third stage, T-Mail, in the migration of the T Documents through my various projects, the data is digitized and automated. One thousand documents were selected from my larger mirror-archive, in which the identity of a sender and receiver is clearly visible. I consider the collection as a kind of pre-war e-mail, composed of a network of international communications. The content of the selected documents was transcribed into a growing database over several years. The database form allows multiple realizations by searching and hyperlinking details of content. 
In the automated display of 'T-Mail', new documents are chosen randomly from the database, a scan of the next document gradually slides into view as various thematic categories and cross-links are activated. Text writings are simultaneously emitted sonically as Morse code, in five different sine wave frequencies that change with each subsequent paragraph. The viewer becomes unnecessary here. Even without a public presence, the computer continually searches and updates information in the database, hereby functioning as a form of 'automatic writing' in which history seems to 'write itself', without further human intervention. Much as the Internet saves and preserves our information traces without our knowledge, scanned handwriting and ASCII text are equally displayed without concern for differences in authenticity, source, or receiver.

The mark of the hand here leaves a public mark, not merely the outward sign of personal, inner, or unique intention. We are presented with the intersections of a person with the bureaucratic, in some sense 'public' systems of recording information. What we also have here, in Dreyblatt's work, is the gesture, and the performance, of making public. These documents were originally parts of non-public, government files, but in the democratic state systems of government (and in the us and the uk, in particular), the government record, often after a 'suitable' delay, becomes the public record, accessible, (potentially) to anyone. Some of the most important political battles going on in the us today involve the current Bush administration's attempts to radically redefine the nature of government, so that what is open to the public will only be what the president explicitly wants the 'public' to see. At one level, Dreyblatt's 'remediation' makes these documents public: it retrieves them from the archive, from a closed, guarded, often locked room, for anyone to see. It also makes visible to us some of the recording and circulating processes that mark the lives of individuals. But more than this, it also exposes to us, and crosses for us, the threshold that separates the closed or 'secret' from the open or public. And it is this very act of crossing the threshold that is at the essence of any construction of a public.

Dreyblatt's Memory Projects, which often have as their point of departure excerpts that he culled from the book Who's Who in Central and East Europe (1935), confront us with a juxtaposition of different moments in time; a juxtaposition of interpretive frameworks, (as biography, history, and other theoretical groundings no longer function as they once did); and a juxtaposition of information collection and display systems (the 'remediation' of the contents of a book by means of a multitude of old and new modes of presentation). A haunting dimension of the project, of course, is our knowledge that so many of the lives whose fragments we read or hear were violently ended in the ensuing years. The various ' $T$ 
Projects' center more on making visible to us some of the enormous systems for observing and recording the traces of a life. While these projects focus on written texts, the various practices of artistic display, from the 'forging' of 'original' documents (using old paper and old typewriters to present documents that look as if they were taken from an archive) to the latest hypertext programs, also belp focus our attention on the hands that type or write or write on or read these documents (reading the printed document is an activity of the hand as well as the eye). Dreyblatt's project, like his musical works, calls attention to the overtones, to the often-unnoticed vibrations and movements that take place 'above' or around the dominant line of information flow. The hands on the document produce overtones, which Dreyblatt now helps us to hear and see.

Artistic practice, in relation to bureaucratic information systems and in relation to new media, often attempts to interrupt a flow of information or to draw attention to the medium (or media) through which information is passing. The attempt is often to obstruct, at least minimally, some operation of power, and to make people more aware of all the shaping and controlling forces that determine what we are presented with. The hope of 'media literacy', for example, is that if the 'consumers' of media are made more aware of the technical, economic, and political processes that determine what they see and hear, they will be less susceptible to its influences. Art is now often a symbolic substitute for the seeming ineffcacy of most 'political' action. Dreyblatt's projects, in contrast, operate less by interrupting or calling attention to the medium (which does not take much artistic skill) than by opening up new possibilities. The power and beauty of new and old media of display fascinate us, but here they also provoke us to ask further questions, and to seek further responses. The works, that engage us in the flow of information and invite us to participate, are unsettling: we continually want to know something more, or to reflect further on some point of connection.

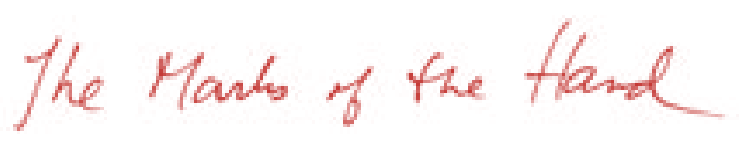

The document in the archive is often seen as a repository of the past, kept and protected for the future. The material seems to be frozen and secure, but the situation is ultimately quite unstable. We find it difficult to choose - as in the preservation of ruins and historical or monumental sites - which document state is actually authentic. The document is migrating through media and technologies much as $\mathrm{Mr}$. $\mathrm{T}$ is migrating geographically with his unstable identity.

Furthermore, the handwritten annotations and signatures marking the documents cannot lead us back to a hand or to a sign of human intention or to the inter- 
document relations that had previously existed. The imprint on paper caused by handheld bureaucratic rubber stamps does not bring us closer to the sender or receivers of these documents, nor do they help us to control their authenticity.

A reconstruction of the life of $\mathrm{Mr}$. T was and is no longer possible, not for the intelligence agents who have to contend with T's multiple identities and continuous issuing of misinformation and downright lies or 'noise', not for the archivists who have to contend with these often conflicting and fragmentary documents, not for the historians who try to make narrative sense of it all, not for me as an artist, and not for the viewer/reader.

The marks of the hand do not function like precious bits of original, authentic material. They will not allow the crime scene investigator to recover the evidence that will determine exactly who is responsible for the death. They will not someday allow us - like the scientists in Jurassic Park, who bring dinosaurs back to life after the discovery, decoding, and regeneration of bits of their genetic material - to reconstruct either a unique life, or the social network that observed and noted a person's activities.

Handwriting no longer betokens the unity of the hand and the individual life, or the intimate connection between the work of the hand and human identity. In the age of technical reproduction, in the archive, in the T Projects, the handwritten indicates Nachträglichkeit rather than original intention. We can no longer hope to reconnect all the varied movements of hands, documents, and identities into an image of a human life, or into a reflection of ourselves.
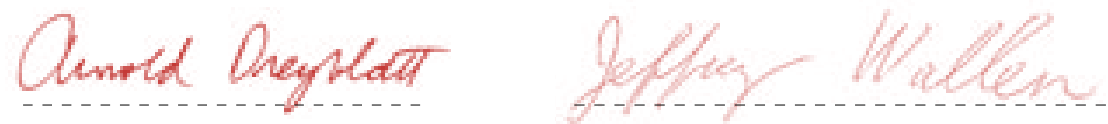
Notes

1. Documents were acquired from the following archives: The Public Record Office and The British Library, London; The National Archives, Washington, DC; Bundesarchiv Koblenz; Politisches Archiv des Auswärtigen Amts, Bonn.
2. See Herman Melville's 'Bartleby the Scrivener' and Franz Kafka's 'Ein Traum' or 'In der Strafkolonie'.

Works cited

Derrida, Jacques, 'Plato's Pharmacy', in: Dissemination. Trans. Barbara Johnson, p. 61-171. Chicago: University of Chicago Press, 1981.

Dreyblatt, Arnold. Who's Who in Central and East Europe 1933: A Hypertext Opera Berlin: DAAD Inventionen '91, 1991.

- T-Docs. Galerie Ozwei, Berlin, 1992.

- Who's Who in Central and East Europe 1933. Berlin: Janus, 1995.

- Memory Arena. In collaboration with the University of Lüneburg, Germany, 1996. Available at: <http://dok.uni-lueneburg.de/memory>.

- T-Mail. Hamburger Bahnhof Museum for Contemporary Art, Berlin, 1999.

- Aus den Archiven // From the Archives. Heidelberg: Kehrer, 2003.

- T.Mail. In collaboration with the University of Lüneburg, Germany, 2005. Available at: <http://www.uni-lueneburg.de/tmail>.

- Inschriften // Inscriptions. Frankfurt am Main: Jewish Museum, 2005.

Glater, Jonathan, 'Stewart Stock Case Is Jolted by Charge that an Agent Lied', in: New York Times. May 22, 2004. Available at: <www.nytimes.com/2004/05/22/business/media/ 22MARThtml? ex=1086262448\&ei=1\&en=d01237fda1 add69e>.

Kafka, Franz, Sämtliche Erzählungen. Frankfurt am Main: Fischer, 1977 (1935).

Melville, Herman, 'Bartleby, the Scrivener: A Story of Wall Street', in: Melville: Moby-Dick, Billy Budd, and other Writings. New York: Library of America, 2000.

Who's Who in Central and East Europe. Stephen Taylor (ed.), Zürich: Central European Times Publishing, 1935 (1933-34). 


\section{Faithfully Submitted:} The Logic of the

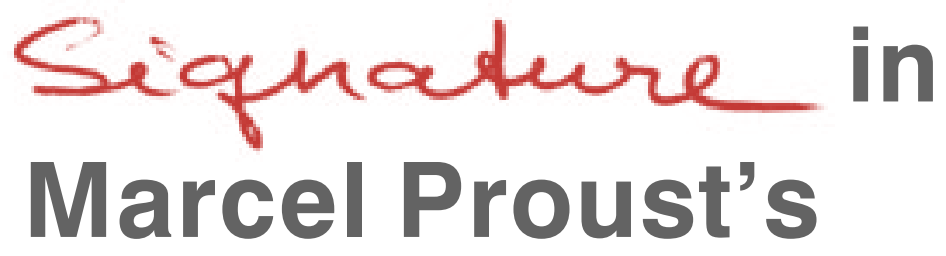

\section{A la recherche}

Proust, known for having handwritten most of the 4,000 pages of his A la recherche $d u$ temps perdu, an essential work of modernist narrative prose, lying in bed on loose pieces of paper, had terrible handwriting. Small, densely written on any scrap of paper that he could lay his hands on, full of corrections, the augustly named 'manuscripts' make the task of putting these scraps in printed form a daunting one. The editors of the two successive Pléiade editions, one in three volumes in 1954 and one in four volumes with ample editorial notes in 1987-89, have accomplished a seemingly impossible task, monumentalizing what once threatened to disappear for want of takers.

It is not as if, oblivious of posterity, Proust was indifferent or oblivious to the adventure of the lines and curves produced by pen on paper miraculously becoming 'language' and, in the best case scenario, literature. At a few key points, this handwritten novel ponders the status of handwriting: its aesthetics, its readability, its delayed arrival. Together, these three aspects of handwriting - the way it looks, its capacity to communicate, and the time it actually takes to be read - which Proust explores in his musings on the signature of a friend, lie at the heart of narrative's extraordinary ability to create visual images. This ability constitutes more than the contested area between poetry and painting. Under Proust's hands, it becomes an emblem of what matters in the artistic endeavor beyond such rivalry. In this paper, I will briefly outline these stakes by attending to the signature that the Proustian narrator reads, misreads, and retrospectively incarnates.

Proust's work loosely centers around the relationship with two would-be lovers, the love of his young years, Gilberte, daughter of Charles Swann and Odette, 
and later, the somewhat vulgar beach girl, Albertine, who lived with him as a virtual prisoner, until she ran away and died in a riding accident. In no other passage of Proust's novel are the paradoxical implications of the literary image made clearer than in the repeated mise en abyme in which the signature of a childhood friend of the hero of La recherche, Gilberte, is described graphically. What is a written image, how can it be read? This paper is dedicated to this question of method, which is developed by means of a 'visual' reading of handwriting in Proust. I will explore the relations between text and image in this author's work through a key image of their complexity; yet, there will be no reduction of the gulf separating the properly visual domain from the domain of language.

The first feature of the signature of the narrator's childhood sweetheart Gilberte - herself the image of the taboo placed on the name in that she rejects and changes her own - is an aesthetic one. Gilberte writes both badly, in other words illegibly, and well, since she creates a beautiful form. Emblematic of the difficulty of seeing, which only grows with desire and closer inspection, the first description of this signature suggests an extreme illegibility due to an excess of pen strokes. The effect of this signature is characterized by a delayed action, an incomprehensible Nachträglichkeit where the joy that the narrator should have felt in receiving a note from his dear friend is not instantaneous. Much later, when the narrator is in Venice with his mother, the same signature leads him to think that Albertine, whom he knows to be dead, has been resuscitated. All of this would come close to being incoherent if it were not precisely for the question of what is involved in image-writing, that is, in a 'flat' writing. This is a writing that neither carves nor scratches, as in etching, nor obeys the modernist norms of aesthetics, readability, and temporal delay mentioned above. Instead, flat writing is something that Proust appears to envision as placed between writing proper and abstract visual expression, detached from the writer's intention and inaccessible to its addressee.

Gilberte's signature - or is it Albertine's? - is the emblematic instance of flat writing. It is, indeed, its exemplary representation. The hero has just recently come back into contact with Gilberte, having brushed against her, watched her, and then been introduced to her without having recognized her as his childhood friend. When he receives the telegram in Venice, logically speaking he does not see the signature of his correspondent, as is the case with all telegrams. He first attributes the telegram to Albertine, his long-term obsessive love object and focus of jealousy who, at that point, is already dead. Again, Nachträglichkeit intervenes and it takes him some time to 'recognize' the telegram as coming from Gilberte. Nevertheless, and despite his earlier failure to recognize her face, he is able to describe Gilberte's signature, which he saw once as a child, without seeing it, and in such minute detail that all concerns for plausibility are suspended (3.671/ IV 235). ${ }^{\text {I }}$

With a keen sense of the primary property of writing, he acknowledges in this passage that the signature is the paradoxical sign that guarantees the authenticity and the originality of the subject by virtues of the latter's absence. In this respect, it is the most characteristic index, seductive as this type of sign tends to be. The signature is one of those 'traces' of a human individual that sets in motion the desire to trace. This desire is the motor of narrative, but one that also informs the persistent 
presence of narrative in sciences such as history, medicine, and psychoanalysis. Thus, a signature is in and of itself a mise en abyme of narrative.

Carlo Ginzburg's seminal article on clues remains key to our understanding of how the kind of thinking based on a narrativity that is informed by the index, and of which the authenticating value of handwriting is a significant instance, has been a long-standing presence in the world of the sciences and the humanities alike (1983). In this article, the historian analyzes the kind of reasoning that underlies the notion of the signature itself, and in its wake, the discourse of the 'hand' that continues to predominate in art historical connoisseurship. Quoting from the 17th-century medical doctor and art collector Giulio Mancini, Ginzburg establishes a parallel between painting and writing:

And these parts of a painting are like strokes of the pen and flourishes in handwriting, which need the master's sure and resolute touch. The same care should be taken to look for particularly bold or brilliant strokes, which the master throws off with an assurance that cannot be matched; for instance in the folds and glints of drapes, which may have more to do with the master's bold imagination than with the truth of how they actually hung. (Mancini, 1956-57, 134, quoted in Ginzburg, 1983, 96)

And Ginzburg develops this parallel between painting and writing into a persuasive theory of reasoning by way of the symptom or involuntary index. Thus, the detail is more decisive than the overall content. Mancini focused on ears, fingernails, and other anatomical details that could easily be overlooked when copying a painting because a certain automatism is in operation. Reading in detail becomes reading 'flat writing' when this automatism comes to stand between the reader and comprehension, and the written text becomes an (abstract) visual image, rather than a readable message.

Yet the signature, like all handwriting and painting, is also capable of being falsified. This turns the index into an icon. This possibility is in the nature of the sign, and distinguishes signs from their referents. By first attributing the telegram erroneously to the dead Albertine, Proust foregrounds that inherent capacity to deceive. By means of the graphic signature of Gilberte/Albertine, the imaginary graphics and the image of grammè, the importance of the visual for Proustian poetics is sketched out and 'signs itself'. To sum this up briefly: handwriting possesses the virtue of flatness.

To understand the significance of flatness, we must look at what is said about the effect of Gilberte's name, which is invested with all the charges associated with naming. Gilberte's signature transforms into a dis-figure of writing. By extension, the name itself, so heavily invested with memory in Proust's universe, transforms from the label of the person into the latter's breezing presence-absence in transitory, fleeting, ungraspable alterity. In the following passage, this function of the name is quite clearly evoked: 
The name of Gilberte passed close by me, evoking all the more forcefully the girl whom it labelled in that it did not merely refer to her, as one speaks of someone in his absence, but was directly addressed to her; it passed thus close by me, in action so to speak, with a force that increased with the curve of its trajectory and the proximity of its target... (1.428/I 387)

In the first part, the description of the name resembles a pragmatic theory of the sign in general; when 'in action' the name becomes a projectile, a weapon, the relevant characteristic of which is its curved flight. But a projectile presupposes a distance, 'a depth'. The object projected comes from the inside out, propelled with force. If Gilberte's name stemmed from the inside, this dimension of the name would place in peril the whole delicate enterprise in which the text is involved. But as signature - authenticated through the flourishes and excess of pen strokes that escape legibility - the name can serve the philosophy of flatness lying over the literary work.

The narrator of La recherche 'explains' the philosophical implications of this poetic in an often-quoted passage: 'How often, when driving, do we not come upon a bright street beginning a few feet away from us, when what we have actually before our eyes is merely a patch of wall glaringly lit which has given us the mirage of depth!' (2.435/II 712). If depth is a mirage, the visual image, with its two dimensions, which make it in one sense 'flat', holds a truth of a quite different depth. It is worth exploring the consequences of such a paradoxical vision of $A$ la recherche $d u$ temps perdu. Such a reading is based upon the hypothesis that the references to visual images, the frequency and the importance of which for this text have been pointed out many a time, suggest a significance of particularly rich, even fundamental value for the poetics of this work, without, of course, this being thereby the only possible meaning.

The motivation for this significance is not a simple aesthetic preference, nor does it suggest a simple exploration of a particularly rich domain of perception and sensation. The 'flat' image combines multiple stances, multiple needs of not only an affective order, but also a perceptual, epistemological and poetic order. All aspects of Proust's poetics of flatness, such as the scopic pulsion, the obsessional voyeurism, the recurrent interest in visual art, the numerous and frequent metaphors borrowed from the domains of optics and photography, the practice of narrative ambiguity in the descriptions, the visual fantasies, the fascination with flatness and the absence of volume, all these well-known aspects of Proust's work come together within a homogeneous framework.

The term flatness sums up and conceptualizes handwriting in Proust in its insistent and ambiguous quality. On the one hand, it designates by inference the absence of volume and of a third dimension. Thus it emphasizes the disappointing and deceptive nature of fiction as well as of the humanistic illusion: 'mirage of depth'. A literal and concrete flatness is the price paid for a visualization of the diegetic universe of the novel; but it is also the pay-off that buys almost total freedom for the imagination. On the other hand, far from being systematically associated with the exalted aesthetics of art, flatness also tends towards a sense of platitude or banality, 
such as is found in Charles Bovary's conversation which is 'flat like a sidewalk', and in the salons of Mme. Verdurin and the Duchesse de Guermantes where the conversation is also flat in the metaphorical sense of the term.

This particular flatness is the other side of the poetics of the work, which would otherwise suffer irrevocably from 'elevated language'. The principal point is, then, that the tension and the inharmonious resolution of the two meanings of the word 'flatness' constitute a central impulse to Proust's literary project. Handwriting, in its quintessential image of the signature, embodies this project. It is, in the terms of Jean-François Lyotard, an emblematic case of the production, in situ, of the concept of the wordimage, or the figural: a force that 'erodes the distinction between letter and line' (Rodowick 2001, 1)

But literature is a verbal art. The visual domain can only be present within it by means of different subterfuges. The principal means of visualization is, of course, metaphor, which causes something 'to be seen' in a way not revealed by the literal meaning, but only accessible through visualization. Furthermore, represented space is very often depicted by using visual images. The narrator describes what he sees or what he saw when he was younger, and this gives a particular importance to the subject of the gaze, which I refer to as the focalizer. ${ }^{2}$ But, in a doubling of this visualization, that which is described is often not a space or a vision but a visual representation: an image, a painting, an engraving or a photograph. On other occasions, the visualization is not doubled, but, instead, is underlined or intensified. The thing seen is described as if it were seen through a magnifying glass or a telescope, or as a projection from a magic lantern, or the framing of a shot seen through the lens of a camera.

My particular concern is one specific aspect of this composition. It involves a visual image, artistic or banal, explicit or implicit, that becomes the mechanism around which the writing will form or deform itself, such that we can think of this writing as properly visual writing. Rather than endorsing the Lyotardian term of the figural, I wish to foreground the generating aspect of the novel more than the kind of signification or the type of sign itself. This generating aspect that stems from the visual, I call figuration.

While I thus approach La recherche first and foremost as a visual novel, it is also a novel in which the subject is threatened with failure. The signature's vagaries embody the connection between these two aspects. And it is through the bond between visuality and the failure of the subject to sustain itself that, I contend, Proust's text lays out the relevance of the reflections on handwriting to which this volume is devoted. The variability, the pluralization and the breaking-up of the subject leads, as Hubert Damisch would say, to a situation in which 'the subject hangs only by one thread' $(1987,354)$. It is to this thread that the limbs of Proust's puppet-like characters are attached. The novel is written in the first person, but, as we know, je est un autre, 'I is an other'. And by way of a rejoinder to this otherness, it quickly becomes clear that the other is 'I'. The narrator's identification with the three key figures of different masculinities - Charles Swann, an epitome of heterosexual obsession and a model of jealousy, Robert de Saint-Loup whom the narrator invests with idealization, and the often ridiculed yet closest to the 'out' homo- 
sexual, Baron de Charlus - is of the same order as the strange 'flatness' of the character Albertine, who moves on the waves of the hero's desire without ever expressing the slightest will. Like them, she is a projection of the narrator, which remains nonetheless unknowable to him. For although the other is 'I', I remains irreducibly other.

The subject is, then, constantly in danger of being absorbed into the other. Here is where figuration comes to do its work. This threatening of the subject is figured, is given form, in the flat image that the narrator, as focalizer, can both contemplate fully and never know. Reduced to being a flat surface, the image confronts the subject at the limits of vision. From a distance, the spectacle loses all life, the colors disappear and the movement of the sea is stilled. Close up, everything becomes muddled. When the hero leans to kiss the cheek he so desires, he is unable to feel any pleasure in it because he can no longer see it. As Bachelard has already said, in the visual domain, there is a rift between minute detail and clarity.

Flatness is given philosophical 'depth' in its bond with the signature, simultaneously unique and imitable. The problem for the subject is that he wants to develop himself by brushing up against the other, represented in both the external world and human beings. But the other in Proust's work always flees, thus creating a conflict that possesses and defines the subject. This existential predicament is figured in the near-obsessive ponderings of the difficulty of vision. Sometimes shade is a better guarantee of visibility; sometimes volume cannot be grasped, while a flat, even banal, surface has more substance and thus offers a more solid base for the narrator's sensualist epistemology. Philippe Hamon's fundamental work on the notion of exhibition (1989), conceived as a sort of textual architecture, traces this epistemology to the 19 th century. He writes notably:

The world of Michelet's 'brilliant trinkets', of paper, signs, advertisement was also a place where objects were beginning to lose volume and depth. In such a world the great projects of historical and philosophical synthesis and of the collation of the document and the monument no longer seem capable of deploying their principle of all-embracing legibility. Before Marx and Benjamin, Baudelaire... equated this incapacity with the loss of memory's or culture's 'halo' or 'aura'. (125)

Proust, as Antoine Compagnon has felicitously phrased it, is situated 'between two centuries' (1989). At both ends of the dialectical movement, which is itself fleeting, lies the two-dimensional image, the flatness of which appears as a permanent temptation to the subject who desires the total 'possession' of his prisoner, a representation of the subject-desiring-knowledge. But at the same time, in figuring the bond between figuration as writing and the tenuousness of the subject, he can also be read as (proto-)postmodernist.

Thus, Proustian flatness and its figuration in descriptions of signatures has a 'deep' philosophical meaning. More can be made of this; let me spell it out. The two-dimensional image is also, simply, flat; it is also a platitude. I call this characteristic of the image its 'flatness', but constantly keeping in mind the conjunction 
between visuality and banality, which Proust will reveal in all its sublimity. As such, 'flatness' is an image of writing. And it is, at the same time, a model for writing, not as an aesthetics of visual art, but as a literary aesthetics based on 'flatness'. It is also the major issue at stake in writing, its principal difficulty and an inextricable interweaving of affectivity and epistemology. The central desire in this novel, which tells of a coming-to-writing, is to ground the subject. The question is, then, how to resolve the insoluble conflict that makes 'I' other, while all the time rooting, as if in a transplantation, the other in the 'I'. Lastly, 'flatness' is also the metaphor for writing as a graphic art.

Armed with this insight, I return to the detail, since that is what first triggered reflection on the signature. The type of detail that emanates from Proustian flatness, and of which the illegible and detached signature is an emblem, can quite simply be called a dis-figure. It is disfiguring as a result of an excess of form, and as such it is related to denial that is absolutely not necessary and is, therefore, excessive: effective negative surplus, the effect of which is proven by the theorist's insistence upon non-sense. A dis-figure is the visual equivalent of a Freudian denial in that it is a figure of negativity produced by excess. The dis-figure is diametrically opposed to what Georges Didi-Huberman has termed pan or patch (1990). With this term, the French art historian refers to blots in painting that elude form, such as the confused patch of red spilling out of a cushion on the bottom left in Vermeer's Lacemaker.

For Didi-Huberman, the pan is a self-reflexive detail. But, as I have proposed elsewhere, self-reflexivity is not the unified phenomenon Didi-Huberman seems to make it out to be (1991). Lest it remains handicapped by the generalization of that term, my analysis cannot endorse this term without proposing its opposite as well. Hence, I propose to reserve the term patch for more restrictive use on the occasions when self-reflexivity effectively takes on a formless form, as opposed to an excess form. Both of these possibilities are deforming, both are no doubt self-reflexive, but the hypothesis creeping in here is that Proust's text, which is after all an irreducibly literary text, even though it is charged with visuality, needs dis-figures more than it needs patches. Moreover, these needs are over-determined in visual terms.

Hence, now is the moment to reconsider the notion of 'literary visuality'. Figuration is clearly distinguished from figurativeness, which is the usage of tropes and which has been so well analyzed by others. I am attempting, quite differently, to ignore as much as possible the distinction between 'literal' and 'figurative'. Sometimes it is in metaphors or comparisons that figuration begins, and this is the case when a visual image is invested with the figuring function or the power to give form, that is, visual form, to that which follows. But it is not because it is a trope that a word, an evocation or the representation of a thing, will serve as a blueprint for the writing. So where is the visual situated in a literary text, an allographic work that is supposedly independent from its material shape as either handwritten, typed, or printed? How can we read 'visually'? Proust's description of the signature and all the misunderstandings caused by its lack of readability, together 
with its excess of 'beauty' and erratic temporality, entails the need to take this question seriously on the level of literature itself, handwriting's seeming opposite. I am considering Proust's text in this respect under the sign of the Wittgensteinian idea of 'language games'. The concept of image - which implies generally, but not always, an idea of likeness, of resemblance, or of similitude - has in the past been analyzed according to its use in five domains (Mitchell 1985, 9-31). The graphic domain is that of painting, most frequently alleged when Proust's novel is considered in relation to visuality, and photography, which I contend is much more crucial for an understanding of Proustian (dis-)figuration. The optical domain is that of mirrors, projections, lenses, and glasses, the recurrence of which in Proust is utterly significant. A third domain is that of sense data or appearances, which are related to perception. Then there are mental images that are encountered in dreams, memories, ideas and fantasies. Finally, at the other end of the spectrum, is the verbal domain, related to which is the domain of metaphors and descriptions. These domains could be broken down even further. It is a 'family' of concepts.

These five domains are not only strongly represented in Proust, they are also thematized in his work, both as part of the theoretical reflection, the essayistic dimension of the novel, and in the collection of fictional representations that constitute the novelistic dimension of the novel, which I contend to be essentially figuration-based. Their presence is at once strong, constant, and confused. The graphic domain alone 'fills' the work: it causes it to 'swell up' with all the descriptions, evocations, references and allusions related to visual art. But this domain is not only relevant in terms of this semantic or thematic network. Its importance is transformed from being semantic to being syntactic, so to speak, when the images, which are visual objects in this domain, figure the text, informing and forming it by imposing upon it certain developments that, without this link to the visual, would lose much, if not all, of their meaning and their richness. These are the images on which the dis-figure superimposes a self-reflexive dimension.

Gilberte's signature is a mise en abyme of the poetics of flatness. As a condensation of the notion of handwriting, this dis-figure is over-determined by the overwhelming bond with another figuration of handwriting, another instance of Ginzburgian trace as narrative. This emblem is 'inflated with meaning' through the mediation of a category of detail that circulates throughout the whole work, namely allusions to the biblical text, recycled through Racine's play about Esther. The presence of the Book of Esther in La recherche is almost obsessive, and with good reason. It can be explained principally by the insistence upon writing in this particular biblical text. As we know from the Bible, Esther manages to prevent the catastrophe that threatens her people by first concealing her identity, then by revealing it at the right moment, and finally by making the most of the delay implied by writing in order to 'countersign' the death sentence with a decree that nullifies the effectiveness of the time bomb that was Aman's first decree.

In her first appearance in Proust's text, Esther is the figure of yellow, which is the color of her dress in the stained glass in the church at Combray ('the yellow of her dress was spread so unctuously, so thickly...' 1.63/I 60). We know the signifi- 
cance of yellow, especially in this buttery incarnation, since it is the painterly substance that kills the writer Bergotte. But in addition to being a life-saving writer, this mythical figure derives her primacy from the bond between writing and the tenuousness of the subject. For Esther is also the figure who keeps secret the fact of belonging to a particular group, and Proust will develop this in his notion of 'being of them' (en être) which serves as a principle of selection for the elite of the 'accursed race', be it of Jews, artists, or homosexuals. Esther is the figure in the closet that signifies a combination of secrecy and choice (Sedgwick 1990). The third dimension of 'Esther', text and character, but also character-text, is the less well-known but equally relevant dimension introduced by her being the incarnation of the signature as speech act that 'does things with words'. Writing, in this case, is words with a power to initiate action, but the action is delayed. The signature is the word of a specific person 'put down in writing'.

It is because of this aspect of Esther that Proust is able to use this character-author-text in order to integrate aspects of his own literary enterprise with the visual mechanism that underpins it. The poetics of his work must be understood and appreciated in the effects produced by the integration of epistemology with affectivity, sexuality with aesthetics, sensuality with poetics. Writing integrates these elements, and it can do so because of the belatedness that it entails. Significantly, visuality is again key to this insight. In the episode of the 'dance breast to breast', where the narrator is confronted with Albertine's potential lesbianism, the poison, the delayed action of which is related specifically to writing in the Book of Esther, is generated by a problematic of vision that is embodied in failing eyesight.

Proust is, here, a preposterous student of Ginzburg. The almost artificially drawn-out slowness requires that the medical knowledge of the focalizer - the doctor Cottard, both an expert and stupid - from whom the narrator is to learn, is also shortsighted, in all senses of the term. Having forgotten his pince-nez, and being less of an expert on feminine beauty than Marcel, he asks 'Are they pretty, at least? I can't make out their features'. He is, however, the professional of medical clichés that enable him to know, rather than see, that 'they are certainly at the climax of their pleasure'. He underlines that his conclusion is based upon prior knowledge and not upon perception when he adds: 'It is not sufficiently known that women derive most excitement from their breasts.' In the gap between perception, for which the necessary optical instrument is missing, and knowledge, which allows a certain deduction, lies the hesitancy upon which the epistemology of jealousy depends, like a parasite. The details of Odette's account of her activities also play within this gap.

One of the narrator's role models, Charles Swann, who does not believe himself to be duped by the poison administered by his mistress, also does not see the semiotic implications of Odette's speech. Neither true nor false - and is that not the essential characteristic of aesthetic discourse? - Swann accepts it nonetheless in his heart of hearts, and hence becomes more and more worried, uttering the vague statement that grounds truth in coherence when he says: 'that doesn't fit with the fact that she didn't let me in'. But this acceptance fails to acknowledge the force of 
the 'surplus matter and the unfilled gaps', words that echo back in poetic terms to the glass jars in the Vivonne when the surplus itself filled in the gaps (1.183/I 166). These glass jars are very clearly presented as poetic: they are a mise en abyme of the poetics of to-ing and fro-ing associated with what I termed in my book on the subject, the poetics of 'mottling'. They oscillate between being container/signifier/ point-of-comparison and content/signified/thing-compared, which already reveals the distinctions between epistemology, semiotics and poetics, all of which are implied in the scene with the glass jars, as they are in that with Swann and Odette. The text only mentions the first aspect verbatim - the aspect of container/content - and makes allusion to the third aspect with the use of the word 'image'. But the poetic aspect of the glass jars is definitely the one that projects its trace in what follows. As the text continues, the subject, like Rousseau in the Reveries, is substituted for the glass jars. He puts to the test the poetic potential of drifting: 'How often have I watched and longed to imitate... a rower who had shipped his oars and lay flat on his back in the bottom of his boat, letting it drift with the current, seeing nothing but the sky gliding slowly by above him, his face aglow with a foretaste of happiness and peace' (1.186/ I 168). This is the poetic potential of the detail that neither Odette nor Swann can see. Having neither the choice, nor the imagination to do otherwise, the latter can only play the same game as his mistress, pitting the same weapons against her:

and making opportune use of some detail - insignificant but true - which he had accidentally learned, as though it were the sole fragment which he had involuntarily let slip of a complete reconstruction of her daily life which he carried secretly in his mind, he led her to suppose that he was perfectly informed upon matters which in reality he neither knew nor suspected... (1.391/I 353).

'Accidentally learned' is here equal to the unfilled gaps elsewhere. Swann thinks he can do battle with Odette's surplus matter using the complete life that she supposes him to know. Brilliantly playing out their interchangeable and disconcerting roles of author and reader, Swann and Odette reveal the impossibility of finding a resolution by means of details.

The semiotic battle between Swann and Odette will later provide a model for Marcel's jealousy concerning Albertine. The difference is, however, that the latter is a much less substantial character than Odette. From the beginning, long before her escape and her death, this 'fleeting being' is truly in flight. Consequently, the search for details becomes a caricature of Swann's actions. Marcel, who depends upon others' help as he hardly ever leaves his house, embarks upon an interminable to and fro between a suspected detail and a confirmed certitude, only to lose that certitude, and thus have to set off on another expedition. The source of the Nile remains always beyond reach.

And that is all for the better. For, as Doubrovsky puts it, this is how the narrator writes: the writing does not 'reflect' nor does it 'wed' jealousy's movements; it 
is jealous $(1974,90)$. It follows, then, that in order to understand the poetics of the detail in this novel, it is necessary to see which detail determines this jealous quest, this voyage of discovery in search of female pleasure that is always hidden and resistant. The gap that must be filled during this quest is, in fact, that gaping hole, those 'huge blanks' (3.93/III 605) that leave a truth without any leftover fragments, without any kernels hidden deep down, from which the narrator could reconstruct something. The huge blanks are like those on a map that designate uncharted land. Archeology is not a helpful model because the fragment cannot come to the rescue of the detail.

The signature thus traced contains the secret of the hidden identity of the self that is projected onto all the others in whom this 'I' discovers himself. Hence, also, the taboo placed on the firstname of the 'I'. The navel of dreams points towards the outside, towards the future of writing, as opposed to the inside of the maternal body and the time passed by the child in this inside. To write, by drops - of ink, milk, sperm, oysters, sweat, and blood - is an act that introduces 'flatness', an act of 'pressing together', of applying the essence of the self onto a flat leaf base.

The trace of writing is, thus, constantly associated with vital forces, the essence of the self, the spurting outwards that make this male, individuated subject; one who writes the reversible surface onto which he applies himself in successive layers. It leads to the infinite spreading-out of this immense work, the base of which is provided by the book. This book is flat, but it is inflated with meaning by means of these residues of observation.

Thinking back to the illegible writing, the unformed form, the figure-figuration that resembles the idea of the dis-figure most closely, despite radical differences, we keep being reminded of Gilberte's signature, that dis-figurer of all figurations. As a name 'put down in writing', with its delayed impact and its capacity to visualize text, this dis-figured signature forms the trace of the visual conflict posed by the detail. Seen from close-up, it is broken into letters, which are in turn fragmented into different strokes. Seen from afar, scattered throughout the work and contained within the illegible name of Marcel's two great loves, now both dead, this signature remains irreducibly broken. It is a tenacious dis-figure, and, as such, like a mise en abyme it inscribes the needs of the novel.

In order that the detail function in a way other than by detailing; in order that it help enlarge or insert - rather than detach - the infinitely small into general laws, there must also be a certain delicacy of form and quivering of flatness and mobility, of light and fragility. It is visual, it is an image, but it is also something else. This something else we find in the realm of photography, an art that is both flat and banal, that is able to enlarge the detail, to capture the past, and to figure movement.

In the scene from the Duchess of Guermantes's salon in 'La fugitive/Albertine disparue', at the moment when the narrator, suffering from amnesia, is to be introduced to the very person who was the great love of his youth, he reflects on the deceptive and disappointing nature of both writing and photography. In the 1954 Pléiade edition of La recherche, the sentence is as follows: 
Our mistake is to present things as they are, names as they are written, people as photography and psychology give an unalterable notion of them. (III 573)

Or according to the Tadié edition:

Our mistake lies in supposing that things present themselves habitually as they really are, names as they are written, people as photography and psychology give an unalterable notion of them. (3.585/IV 153; translation adapted $)^{3}$

This reflection is offered as an explanation for the bizarre fact that the narrator had 'mis-corrected' the name that the concierge had already misheard and written incorrectly. The fact that Gilberte had stared at him, and that he had brushed up against her, taking her to be a tart without recognizing her, appears in no way strange to him. For this reflection to take place, it is forgetfulness, rather than the contrary, that is necessary.

Unexpectedly, the deciphering efforts of the successive generations of editors of Proust's challenging handwriting received profound philosophical relevance. In the first Pléiade edition, the verb 'présenter' was not reflexive, making it a question of presentation, that is, of photography as a means, as a medium of communication. In the Tadié edition it becomes a question of the belief that the frequent use of the medium has been established as a routine element of culture. Between these two versions we can see the difference between a behavioral pattern and a conviction, between a culture and a religion.

The patch that in-forms is superimposed in this work with the dis-figure that de-forms. The way in which invisibility also becomes a mise en abyme in Gilberte's signature, which is illegible because it is too detailed, is explained by the fact that this signature signifies too much at one time. It provides too many forms and thereby shields the sign from the desperate search for meaning. In this, the signature, far from authenticating a subject who has no substance and lives only off the rubbing against alterity, dis-figures subjectivity through excess as denial - through a postmodern dis-belief in authenticity that pre-posterously confirms Derrida's critique of the signature. Rather than inscription of uniqueness, then, the signature signals a flatness that, in turn, gives shape to the fabric of the culture in which it occurs.

If, then, Ginzburg ends his article on the conceptual metaphor of inquiry as fabric, he inevitably arrives at a critique of the politics of that kind of tracing that handwriting stands for. He describes the protection of civil society by means of securing individual uniqueness and its recognizability, that, I may add, is today re-invigorated by the us's fingerprinting practice that polices all foreigners. On the final page, he mentions Proust as 'a rigorous example of the application of this conjectural paradigm' (109). What he does not seem aware of is how Proust applies the paradigm without illusions of individuation. Instead, the signature, and the enter- 
I 62

Sign Here! / Faithfully submitted

prise of jealousy for whose failure it stands, is firmly illegible - in all senses: as name, as authenticity, and as depth. Flatness, instead, lays out a sociality beyond these traps of humanism.

SIGN HERE! 
Notes

1. I am quoting from the four-volume French edition of $A$ la recherche du temps perdu. edited under the direction of JeanYves Tadié. Paris: Gallimard, Bibliothèque de la Pléiade, 1987-89. (Trans. by C.K. Scott-Moncrieff and Terence Kilmartin as Remembrance of Things Past. London: Penguin Books, 1981).
2. See Bal (1997) for this term, which refers to the perceptual or interpretive relation between subject-seeing and what is seen.

3. The Tadié edition, which has incorporated new manuscripts, appeared after the English translation.

\section{Works cited}

Bal, Mieke. Reading 'Rembrandt': Beyond the Word-Image Opposition. New York: Cambridge University Press, 1991.

- The Mottled Screen: Reading Proust Visually. Trans. Anna-Louise Milne. Stanford: Stanford University Press, 1997.

- Narratology: Introduction to the Theory of Narrative. Trans. Christine van Boheemen. Toronto: University of Toronto Press, 1997.

Compagnon, Antoine. Proust Between Two Centuries. Trans. Richard E. Goodkin. New York: Columbia University Press, 1992.

Damisch, Hubert. The Origin of Perspective. Trans. John Goodman. Cambridge: MIT Press, 1994. Originally published as L'origine de la perspective. Paris: Flammarion, 1987.

Didi-Huberman, Georges. 'Appendice: question de détail, question de pan', in: Devant l'image: Question posée aux fins d'une historie de l'art. Paris: Editions de Minuit, 271-318, 1990.

Doubrovsky, Serge. La place de la madeleine: Ecriture et fantasme chez Proust. Paris: Mercure de France, 1974.

Ginzburg, Carlo. 'Clues: Morelli, Freud, and Sherlock Holmes', in: Umberto Eco and Thomas A. Sebeok (eds.), The Sign of Three: Dupin, Holmes, Peirce, 81-118. Bloomington: Indiana University Press, 1983.

Hamon, Philippe. Expositions: Literature and Architecture in Nineteenth-Century France. Trans. Katia Sainson-Frank and Lisa Maquire; intro. Richard Sieburth. Berkeley: University of California Press, 1992.

Proust, Marcel. A l'ombre des jeunes filles en fleurs, A la recherche du temps perdu vol. 1. Edition établie et présentée par Pierre Clarac et André Ferré. Paris, Gallimard, 1954 [orig. 1918]. (English: Within a Budding Grove: Remembrance of Things Past vol. 1. London: Penguin, 1954).

- Contre Saint-Beuve (including Pastiches et mélanges and Essais et articles). Edition established by Pierre Clarac with the collaboration of Yves Sandre. Paris: Gallimard, Bibliothèque de la Pléiade, 1971.

- A la recherche du temps perdu. 4 vols. edited under the direction of Jean-Yves Tadié. Paris: Gallimard, Bibliothèque de la Pléiade, 1987-89.

Sedgwick, Eve Kosofsky. Epistemology of the Closet. Berkeley and Los Angeles: University of California Press, 1990. 


\section{(Hand)writing Film History: Saul Bass} Draws Martin

\section{Scorsese in a Title Sequence and writes} his name underneath

Something starts to hatch in the upper right-hand part of the screen. White lines on black. No pen, no hand. Just a movement and a sound. The movement is controlled, it is up to something. A curve, it is now drawing an ear. Figurative drawing. A forehead (the hatched elements are the hair), again hatching: an eyebrow, nose (dot, dot, comma, dash), stop - handwriting, we follow the words: 'A personal journey', underlined (this is a headline), music starts, new paragraph, 'with Martin Scorsese', new paragraph, 'through American Movies'.

Martin Scorsese's contribution to the series of national cinema documentaries 'celebrating the moving image' for the cinema centenary, begins with a title sequence that reflects on the status of his signature. Even before the first quote, before the first clip from Minelli's The Bad and the Beautiful, in which the producer (played by Kirk Douglas) states 'To be a director, you must have imagination', and the director (played by Ivan Triesault) answers 'Whose imagination, Mr. Shields?', Scorsese has been introduced to the viewer by someone else's imagination. Somebody else has provided Scorsese's signature for his film. The personal statement that is about to follow, made of materials from other films, takes off with the display of hybridity as the very core of filmic authorship.

In the $1994 \mathrm{TV}$ production on cinema history, that somebody-else-who-signs is Saul Bass, the most famous designer and director of film title sequences in film history: 
I've had the honor and the opportunity to work with Saul and Elaine Bass on three of my pictures - GoodFellas, Cape Fear, and The Age of Innocence. We're now working on a fourth, Casino. It has been a dream of mine to work with Saul Bass ever since I tried to capture his style in my own 'imaginary' movie titles which I drew at ages 12 and 15 in a composition book I kept hidden at home. I feel very fortunate to have had that dream realized. (Scorsese 1996, 3)

Scorsese's dream of his own self-designed signature in the style of another, which he has kept hidden at home since puberty, really comes true in the latest of his series of collaborations with Saul Bass, the fifth one, which, in a film taking stock of the past, is, not without irony, also Bass's last title design in general. That the truth differs slightly from the dream - it is Saul Bass's style that tries to capture Scorsese's signature in an on-screen composition sketch - does not matter that much. Getting personal in this film is a triumph in itself. The very project of the film commissioned by the British Film Institute (BFI), the largest film archive in the world, places Scorsese at the center of the center, the number one filmmaker in the world's leading film industry: the one who comes to mind first, both as Hollywood's main representative and its best informant. Film and its reading coincide:

[T] he British Film Institute commissioned The Century of Cinema, an ambitious series of documentaries in which the world's leading filmmakers were asked to interpret their native country's cinema. From day one there was no question that the American segment belonged to Martin Scorsese. (Wilson, 8)

The film we are about to see is a film about belonging. We will see the writing of film history, and the making of its writing. To get the job done, the handpicked director handpicks three close collaborators: an established film historian and documentary filmmaker, a famous composer and a famous title designer. ${ }^{I}$

Why does a television production attach that much importance to the title sequence? ${ }^{2}$ That Saul Bass designs a television title sequence (which happens to be his only work for television aside from the title design for the television series Alcoa Premiere in 1961) for a documentary about the history of Hollywood appears at first sight to be one of the ironies of film history, taking into account that the phenomenon of independent designers such as Saul Bass came into being in the late fifties, above all as the result of the media rivalry between cinema and television. At that time, film studios tried to compete with television by raising the production values of their productions, inter alia, by increasingly favoring the more expensive independent graphic designers for the title design of their films over designers from the studio-owned title houses that had provided most of the title sequences for the film industry up to then. Now, years later, it is television that keeps the memory of cinema alive. Hollywood itself had already discovered the structural importance of title sequence design at a very early stage. The first company specializing in film title design dates back to 1902 and around 1904 'the practice was adopted as commonplace' (Allison, 115). 
Classical narration usually begins before the action does. True, the credits sequence can be seen as a realm of graphic play, an opening which is relatively 'open' to non-narrational elements.... Yet the classical Hollywood film typically uses the credits sequence to initiate the film's narration. Even these forty to ninety seconds cannot be wasted. Furthermore, in these moments the narration is self-conscious to a high degree. (Bordwell, Staiger, and Thompson 1985, 25)

Saul Bass's film-within-a-film of 1994 capitalizes on this second moment, which has become more self-evident for today's movie-going audiences. His introduction to Scorsese's documentary flashback of US cinema history is made in a way that reflects on the very linchpin of auteur criticism, which is the concept of the 'signature' of a film that was adopted by film critics such as Andrew Sarris in the United States at the same time that Scorsese was just beginning his film career. In the early 1960s, the talk of the 'signature' of a film had both an emancipatory and a classificatory function. On the one hand, it endeavored to put film on a level with the traditional arts. It distributed value and it did so by making use of the traditional parameters of the literary system. The various forms of decision making in a filmic structure, which is fundamentally based on the division of labor, are to be traced back to one origin: the director:

The second premise of the auteur theory is the distinguishable personality of the director as a criterion of value. Over a group of films, a director must exhibit certain recurring characteristics of style which serve as his signature. (Sarris, 662)

With this move, working for an industry ceases to be an obstacle to creating art. Hollywood is capable of generating masterpieces and genius as well:

His [Andrew Sarris's] writings led me to see the genius in American movies at a time when the cinema was considered a mindless form of entertainment, worthy of serious attention only if it came from Europe or Asia. Sarris's... wonderful ability to articulate for me and many others as well what we unconsciously felt about the styles of various directors played a truly pivotal role in my life. (Scorsese 2001, vii)

It is not by chance that it is Scorsese who provides the foreword to a collection of essays in honor of Andrew Sarris, a short message of greeting with the title 'The Fundamental Film Teacher', as it is Scorsese who provides the foreword to the 'Master Series' catalogue of works by Saul Bass:

Saul Bass's reputation as a designer of film is legendary. He has left his indelible signature on a number of pictures by Preminger, Hitchcock, Kubrick, Wyler - among others. It was exciting growing up on movies during the 50s and 60s. That 'growing' entailed a great deal of learning, too. Part 

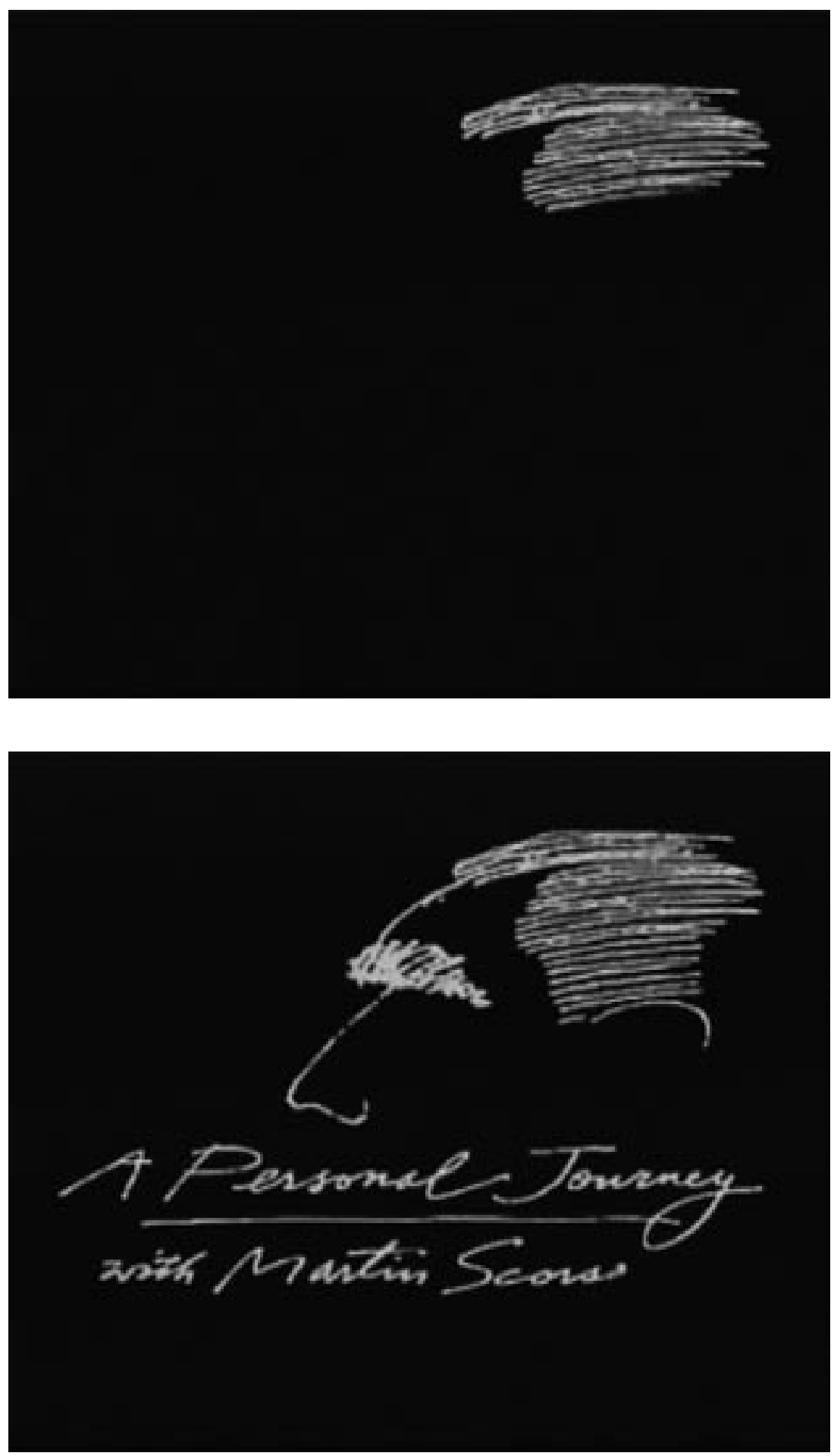
of that excitement and learning was due to the remarkable contribution of Saul Bass in some of the greatest films of those periods. (Scorsese 1996)

The indelible signature of the signature specialist behind each on-screen signature marks the fundamentally schizoid structure of the filmic title sequence. In order to put one's own signature on display one has to hire somebody else whose rendering of your signature also serves as his own: 'Up until his recent death, Saul Bass continued to carve his artistic signature on the movies' (Haskin, 11). Of course, this has major consequences for the notion of authorship. In the movies we are confronted with an author, who cannot even write his own name. The title sequence introduces the name and celebrates it but it never stops at this point. Because there is no single act of signing, the parade of signatures designed to establish authorship contradicts the importance of the single name. With respect to author criticism, the notion of the 'signature' can become a pun: 'It was the only time I ever met him, but I came away from the session with... the auteur's autograph ("Sincerely yours, Andrew Sarris")' (Bordwell 2001, 165).

In what follows, I will attempt to decipher the handwritten signature of that exclusive part of the title sequence that actually names the film A Personal Jour$n e y$, leaving the other credits, which are just white printed characters on black, out of the picture. I will try to follow instead some of the windings of the writing of the name, its various contexts, detours, and allusions because the title sequence of a film is where the metaphorical talk of its 'signature' can be read literally.

Of course, to avoid any misunderstanding, 'handwriting' in film is handwriting in name only. First of all, the single letters are based on contemporary letterforms and '[s]econd, any handwriting that can be transposed into reusable typeface functions fundamentally as a mechanized script' (Kittler, 259).

In the beginning there was the boy's dream of fathering a film, of devising an unambiguous signature of his own for everyone to read. To have his dream of making the dream factory. What do we make of this dream? How does one make films out of dreams?

\section{Screen memories}

One day, in the Interpretation of Dreams, Freud goes to the movies to get some ideas on the right way to bury his father. He sees himself confronted with the paratext:

During the night before my father's funeral I had a dream of a printed notice, placard or poster - rather like the notices forbidding one to smoke in railway waiting-rooms - on which appeared either

'You are requested to close the eyes'

or, 'You are requested to close an eye'. 
I usually write this in the form:

'You are requested to close ${ }_{\text {an }}^{\text {the }}$

... In a few instances the difficulty of representing an alternative is got over by dividing the dream into two pieces of equal length. (Freud, 352-353)

Some time before the start of the home movie, before his father's burial, Freud passes the time by staring at a framed space upon the wall on which an inscription of the law unfolds. It is night time, the setting is the auditorium where one can see L'Arrivée d'un train - the fantasized primal scene of cinema, in which the object leaves the screen and enters the space of the spectator. ${ }^{3}$ Freud is familiar with these kinds of accidents. They triggered his Studies on Hysteria a few years previously. 4 Now he is waiting in the darkness and sees the projection of an alternative version of the story in the parade of letters before the story unfolds. For the burial of his father, Freud has asked cinema for help. What he is dreaming of are different modes of representation. What he finds is the simultaneity of two conflicting versions represented by the closing of (an) eye(s). Dreaming, imagining, focusing, allowing, Freud dreams of the funeral in alternate versions before the actual funeral. He dreams up a new editing process. Closing father's eyes, overlooking general demands, opening alternate points of view. The printed letter as dream content? The letter is a rebus?

One day in the 1940s in A Personal Journey Through American Movies, Martin Scorsese goes to the movies for the first time without his father. He sees himself confronted with the paratext:

I remember quite clearly - it was 1946 and I was four years old - when my mother took me to see King Vidor's Duel in the Sun. I was fanatical about Westerns. My father usually took me to see them, but this time my mother did. The movie had been condemned by the Church. 'Lust in the Dust', they dubbed it. I guess she used me as an excuse to see it herself. From the opening titles I was mesmerized. The bright blasts of deliriously vibrant color, the gunshots, the savage intensity of the music, the burning sun, the overt sexuality. A flawed film, maybe. Yet the hallucinatory quality of the imagery has never weakened for me over the years.... It was all quite overpowering. Frightening too..... I covered my eyes through most of it..... I didn't know by then, but in 1946, Hollywood had reached its zenith. (Scorsese 1997, 14)

In the film that reveals his authorship at its zenith, Martin Scorsese gets personal by telling the story of how he was taken by the hand for the first time. The construction of both the national film canon and his signature begins with a series of images that reflects on the relationship between writing, reading and the cinema. 
The opening titles had already given us the name, handwriting, and an author portrait as a first hint. This is followed by the first filmic quote of the compilation film from Minelli's The Bad and the Beautiful, which furnishes a motto concerning the ethics of production. Then we see the narrator (Scorsese) sitting in a chair, the author portrait from the beginning is translated into a frame narrative, a point of view. This is a documentary. We are listening to that person's story now. In the beginning there is a lack. Nothing is written:

there was nothing really available that I could find written about film.... [A]ll I had at my disposal to experience then were these black-and-white stills. I would fantasize about them, they would play into my dreams. And I was so tempted to steal some of these pictures. (Scorsese 1997, 14)

Nothing is really moving yet (this is still the story being told.) Flicking through the stills of a film book from the public library, zooming in on one, Scorsese arrives at his primal scene. It is another title sequence, this time in color. We're almost back again to where we have already been. It is at the place of the filmic imprint (outside of the diegesis) that the film gets personal.

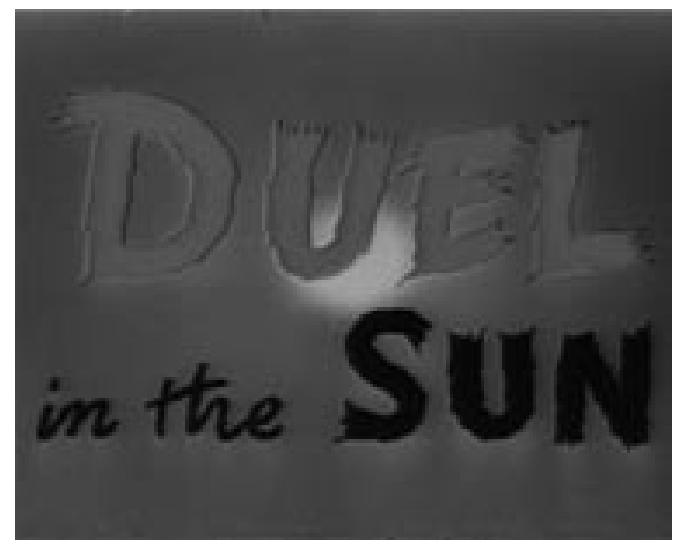

2. Duel in the Sun 1946

What four-year-old Little Martin sees within this scene of oedipal nostalgia is not so much horses, but something that is too much for him. It is not the first time he has been to the movies ('I was fanatical about Westerns' (Scorsese 1997, 14) but this time he literally does not know where he is. Is he on screen or is he in the audience?5 Little Martin is overpowered even before the diegesis unfolds. He is already paralyzed by the title sequence, by the force of its various elements. How is this 
possible? The title sequence merely consists of letters on a background, scrolling upwards. It looks quite simple. It works because it anticipates and doubles the instability of the agencies of identification. On the one hand, the opening credits that disrupt the dark space of the movie theater address Martin as a spectator in the usual way: 'Get ready. Time to identify'. On the other hand, this credit sequence stages projection itself by generating a metaphor for it: the screen seems to reflect the projection backwards. What Little Martin is staring at, sitting in the cinema: shiny spot in the background of the image, the source of light, the 'burning sun', blurry as if behind glass, the bulb of the projector. Seeing the projection and keeping the name in mind become one:

[T] he first film I remember seeing by name: Duel in the Sun. The safety of the darkness of the theater was suddenly shattered by a bright blast of deliriously vibrant color followed by gunshots - the opening credits of Duel in the Sun. (Scorsese 1995, 141)

The first 32 seconds about this film that one could see in movie theaters - the teaser - had already introduced a form of violence that is specific to the beam of light: Coming Soon! BANG! The shiny spot, which looked safe while sitting in the background, is now suddenly animated and projected into the foreground like a projectile, only to present the encapsulated letters of the titles of the film. The underlying gunshots reinforce one's sudden subjection to the moving image. This metaphor is more about a technical than a narrative device but with the projectionist's beam of light metaphorically reversed, it is Martin who is on the screen. ${ }^{6} \mathrm{He}$ is seen. And the parading of the letters and the law plays out across his face, shrouded in orange. The mirroring screen has itself become an indicator, a litmus paper turning 'orange'.7 BIG ORANGE: Hollywood! (At its zenith). ${ }^{8}$

What the opening credits of Duel in the Sun accentuate is not so much the film's indebtedness to Gone with the Wind or the 'heat' of the scene, the famous 'shooting directly into the sun... which had spotlights shining right into the camera' (Higham, 262), but the 'heat' of projection (which might even destroy the film.) The Dimitri Tiomkin's soundtrack climaxes exactly at that moment when the credit 'Color Director: Natalie Kalmus' appears, thus emphasizing the coloring of the film. 'Technicolor' has already had a dramatic entry before: 'DAvid o. SELZNICK presents his production in TECHNICOLOR OF KING VIDOR's.'

The title-sequence of Duel in the Sun becomes delirious, vibrant, savage, intense, burning, sexual, hallucinatory by means of the mirror-effect that is dramatically dyed. What it says is that everything that follows is the effect of projection and of Technicolor. And this is not just part of a story. In every film the first duel in the sun of the projector bulb is the duel between the title sequence and the film. Freud's 'either-or' way of representing, characteristic of dream work, can be found here. A relation that is filled with tension. Each film is divided into at least two parts and has a 'making-of' film in its beginning. Each film has its own story already told and read, before the story unfolds. Each film provides us with a version of itself as something to start with: 
While the film systematically speaks of something other than itself (of an anecdote for instance), the title sequence speaks of nothing else but the film, thus exposing what the film has carefully kept hidden. A scandalous competition. (Gardies, 86)

The names of the main cast members in the Duel in the Sun-title are neither printed nor handwritten. They are painted. While the frayed typeface dramatizes the plot, illustrating the inner conflicts - 'Duel' and 'Sun' compete in size, with 'Duel' in red, one hears the sound of shooting beneath - it also directs attention to the variations and hierarchies in the parade of credits, which is led by the producer and finished off by the director. That which brings 'Duel' and 'Sun' together is written in script ('in the'), locating the projection, making it personal. This is not by chance.

Hollywood has always specialized in reflections of the signature. How does this work? How does film visualize signatures? Let us not harbor any illusions about it. In Hollywood's classical cinema, the person who signs the film first, quite unambiguously, is the producer. His name is the one that starts the film. The development of more flexible production units in Hollywood in 1931 had triggered certain stylizations of the figure of the producer in title sequences such as, among others, variations in the typeface of the producer credit. ${ }^{9}$ (The Samuel Goldwyn signature is just one example of this). But there have also been title sequences that challenged the prominent position of the producer credit. King Vidor was one of the first Hollywood directors who entered into a creative rivalry with the producer, by individualizing his own credit also via handwriting, in contrast to the printed characters of the other credits (i.e., The Texas Rangers, in 1936). In 1946, there was no opportunity for a stylized signature yet: ' $[\mathrm{t}]$ here was Directors Guild arbitration before Vidor emerged with sole credit’ (Durgnat, 239).

What about A Personal Journey, which displays this title sequence shortly after its own title sequence? Who is actually signing this film? After all, it is the handwriting that is supposed to reveal the film as personal. The handwriting transcribes the 'personal' of the title. The signature on the main title itself simply reads 'Martin Scorsese'. But is this a signature? A name emerges from the handwriting that we follow with our eyes. It is part of the title. The writing of the name, however, takes place in a specially allocated space, right underneath a line that sets it apart from the personal journey. That a name is introduced by 'with' does not irritate us. We know this from actors' credits (diary titles may also look like this). The combination of an identifiable author image and witnessed handwriting suggests both that it is and is not a signature. Who is writing here? The handwriting resembles that of Saul Bass. We remember his handwriting from Why Man Creates, the Academy Award-winning short film from 1968 . We can identify it, yet we see neither hands nor writing implements. Perhaps somebody else is imitating Bass's handwriting. Scorsese? Is this the director's credit at all? A Personal Journey with Martin Scorsese through American Movies is made by two directors. ${ }^{\text {IO }}$ And there is still the director's credit to follow. The personal journey through American movies begins with a signature that writes itself. In place of the little pictograms between the segments of the Duel in the Sun title that make explicit the genre or the field of spe- 
cialization - a guitar, a pair of pistols, a pistol - this film presents a face. Physiognomy substitutes the ironic bull skull above King Vidor's name (which is both a genre and a property sign - 'you are entering the property of King Vidor'). The main title card of the BFI film is organized like an emblem. The pictura is an author portrait, a sketch, a caricature - (it takes only one-half of the face to recognize it) - the subscriptio is the title of the film. ${ }^{I I}$ The upper part of the frame with the upper part of the face supplies an ironic commentary on the author's function in the film. This is where the brain is, this is where the eyes are (taken literally, it is also a pun on the notion of the 'frontispiece'). The fact that the main title card and the director's (possessory?) credit coincide is rare in film history. The practice goes back to the main title design of early cinema that provided all of the information on a single card. The characterization of the star by his or her physiognomy is also typical of that time:

Before 1917, films commonly introduced characters in ways that called attention to the act of narration. An expository title would name and describe the character and attach the actor's name; then a shot might show the character striking a pose in a non-diegetic setting (e.g., a theatre stage). After several characters were introduced this way, the fictional action would begin. After 1917, such signs of narration diminished. Characters would be introduced upon their first appearance in the action. Overt commentary in the titles ('Max, a Bully') would be replaced by images of the character enacting typical behavior (e.g., Max kicking a dog). (Bordwell, 27)

In 1994, in the personal journey through American movies, one comes across a myriad of famous actors, but only one star: Martin having his name written. Not many directors could make it as stars (and in so personal a manner) in a title sequence. Only very few are immediately recognizable by a larger audience. ${ }^{\mathrm{I2}} \mathrm{By}$ presenting the director as the star and main attraction, Bass's title builds up a lineage. In the context of television history, it quotes (and inverts) the famous stylized profile that opened the Alfred Hitchcock Presents television series of the 1950s and 1960s. The quotation is ambivalent, however because it does not start only one family. Instead, it introduces Hitchcock not only as the director, and Scorses's predecessor, and other seminal Hollywood figures that come to mind, it also introduces Hitchcock as title designer (the position he began his film career with) as both the predecessor of Saul Bass ${ }^{I 3}$ and his most famous employer. ${ }^{I 4}$ The sketch that opened Hitchcock's television show was actually from a Christmas card that Hitchcock designed himself while he was still living in England. As an ironic means of authorization, its eight lines adopted more and more the gesture of a signature, like a grapheme in its own right. The 32-cent Hitchcock postage stamp that appeared as part of the us Postal Service's 'Legends of Hollywood' makes this perfectly clear by repeating the black-and-white photograph of Hitchcock with the stylized profile in the upper-left-hand corner. Hitchcock is able to sign his product with the wavy contours of his own profile. 


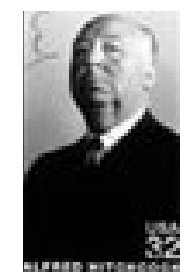

3. Alfred Hitchcock

commemorative stamp,

32-cent,

US Postal Service 1998

Hitchcock, the big rival in who-comes-to-mind-first-with-respect-to-Hollywood, was not particularly on Scorsese's mind during his journey through American film:

At first, we planned to include almost every director that had inspired Marty before he had embraced filmmaking. But there was no room to do justice to them all.... [E]ven today Marty laments the sites we never visited or geniuses we didn't discuss, from Ernst Lubitsch to Alfred Hitchcock. (Wilson, 8)

Unlike Alfred Hitchcock Presents, where each episode would begin with Hitchcock's silhouette profile filling in with black, the lines in the making of 'Scorsese' are white on black. The profile remains transparent. Nothing is filled in. Leaving the Scorsese profile transparent, Bass makes the hidden presence of Hitchcock even more felt: it is a pun that marks an absence. The cursoriness of the sketch that is not allowed to become substance gives the portrait a ghostlike quality.

To celebrate the century of cinema, we have been sent back to school again with a black background, white lines, the scratching sound of the invisible chalk as we learn to read and write all over again. The blackboard on the screen identifies the series of images that is about to follow as readable text. The screen is a slate is a mystic writing pad. All these various writing materials have in common their basic relationship to the concept of the trace. Each sign that shows up on them plays with the relationship between absence and presence, each emerging sign is almost already wiped away at the very moment it shows up. ${ }^{15}$

What does it mean to return to hand-drawn images and handwritten letters on a blackboard in the age of digital composition? On the one hand, it is without a doubt a nostalgic gesture in itself: 


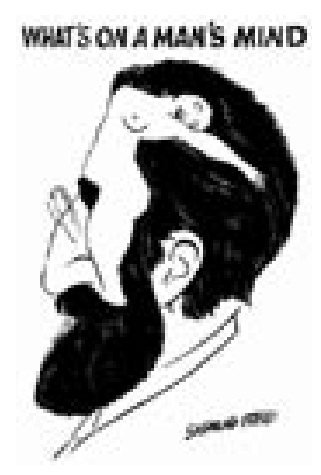

4. What's on a man's mind

There are many threads running between the films, with... Saul and Elaine Bass doing the titles.... [T] he style of their work was extraordinary and we wanted the audience to be very aware of the lineage of this type of film. The sadness for me is that I can't make films in the old style, the studio system style, because I'm a product of a different world and society. (Scorsese, in: Thompson, 174)

On the other hand, the title design of A Personal Journey can also be seen as a pointed move away from the camera and the photographed image as the basis of the filmic image, which is characteristic of digital cinema:

[T] he manual construction of images in digital cinema represents a return to the pro-cinematic practices of the nineteenth century, when images were hand-painted and hand-animated. At the turn of the twentieth century, cinema was to delegate these manual techniques to animation and define itself as a recording medium. As cinema enters the digital age, these techniques are again becoming commonplace in the filmmaking process. Consequently, cinema can no longer be clearly distinguished from animation. It is no longer an indexical media technology but, rather, a subgenre of painting. (Manovich, 295)

The animation both of writing and drawing in this sequence pays tribute to a classic in the history of animation film: the Inklings series by Dave Fleischer. Inklings, issue 12 from 1925 reminds one most of Bass's particular approach to the main title design of a film on film history: Here we see the painted sketch of a bearded man in white brush strokes on a black surface turning into Rin Tin Tin once it is turned 
upside down (which is again reminiscent of another famous picture puzzle: the portrait of Sigmund Freud 'What's on a man's mind?').

The hand-drawn face and name of Martin Scorsese are the focal points of A Personal Journey with Martin Scorsese through American Movies. Due to its emblematic character, Bass's personification of the filmic force as a head looking to the side, with the signature underneath, alludes to the history of the studio logo, in particular, the MGM lion. 'Scorsese' is an American, is a lion, is a myth. Turning the studio 'look' into a personal style, he manages to become a trademark of 'Hollywood'. ${ }^{\text {I6 }}$

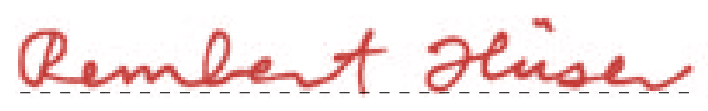

SIGN HERE! 
Notes

1. 'A poet or a painter can be a loner, but the film director has to be, first and foremost, a team player. Particularly in Hollywood' (Scorsese 1997, 20).

2. That it's a work for a television production is probably the reason why this title sequence is not as well known as most of the other Saul Bass title sequences. 'Martin Scorsese's most recent film, Casino, features... the last title sequence that Bass would make before his death' (Haskin 1996, 11).

3. Perhaps the oldest cliché of film history is the reputed reaction of the first audiences to the Lumières' L'Arrivée d'un train en gare de La Ciotat. Spectators were said to have jumped from their seats in terror at the sight of the train coming toward the camera and running beyond its purview (in three-quarters' view) logically 'into' the space of the spectator. Descriptions of such a response in France and elsewhere survive in film history as rhetorical indices of film's initial novelty and the naïveté of the spectator confronted by a two-dimensional, dynamic representation. Gunning sums this up: 'Thus conceived, the myth of initial terror defines film's power as its unprecedented realism, its ability to convince spectators that the moving image was, in fact, palpable and dangerous, bearing towards them with physical impact.' He goes on to refer to this myth as a 'primal scene' informing film theory's view of early film history' (Kirby, 62).

4. 'The railway accident as an agent of traumatic experience occupies an important place in the history of mid- and late19th-century medical and medico-legal discourses over trauma and traumatic disorder. In fact, it can be argued that systematic medical theorization about psychological trauma in the modern West commenced with the responses of midVictorian medical practitioners to the socalled railway spine condition, which was characterized by the manifestation of a variety of physical disorders in otherwise healthy and apparently uninjured railway accident victims. The investigation of this condition led many 19th century surgeons to examine the role of psychological factors - variously referred to as "fright", "terror", or "emotional shock"

- in provoking physical disorders some thirty years before Freud and Breuer considered the matter in Studies on Hysteria' (note: 'Freud's own earliest writings on hysteria and trauma were prompted by the debates over railway accident cases to which he had been exposed in Paris and Berlin in the 1880s' Harrington, 31-32). See also: 'Essentially, the idea that the train image inspires a threatened response terror - and does so as a film image, returns us to the whole history of train travel as a paradigm for cinematic spectatorship based on shock' (Kirby, 62).

5. Little Martin, who is viewing the forbidden movie with his mother, in the first ten minutes sees the mother who is cheating on the father getting shot by the father along with her lover. The father is then hanged for the crime. For Little Martin, who has left his father home alone, there are still 128 minutes of sitting beside his mother left.

6. The trailer, which adds both the producer's logo and little silhouettes in front of a sun that has become bright red, concentrates on the brand name. 'A shot of the Selznick Studios logo (a shingle with the 'Tara' building that served as the studio headquarters in the background) is first seen, accompanied by an epic trombone fanfare. Then a narrator announces, "The studio that made Gone with the Wind brings you...", after which there is a cut to a graphic of the sun with jagged brown lettering inside it that zooms slowly into the foreground until "Duel in the Sun" can be read. Visually, the graphic (on the heels of a picture of Tara) echoes the famous "Gone with the Wind" title silhouette of Scarlett O'Hara on the hill at Tara with the sun behind her (which was also a key image in the GWTW trailer)' (Kernan, 108-109). The title sequence of the film still echoes 'Gone with the Wind', but is stripped of all direct thematic allusions besides the 'sun'. More abstract, it stands by itself.

7. It is Duel in the Sun that inspires Laura Mulvey to 'afterthoughts' on her concept of female spectatorship: 'The fantasy of "action" finds expression through a metaphor of masculinity. Both in the language used by Freud and in the male personifications of desire flanking the female protagonist in the melodrama, this metaphor acts as a strait-jacket, becoming itself an indicator, a litmus paper, of the problems inevitably activated by any attempt to represent the feminine in patriarchal society' (Mulvey, 37). In this film, the metaphor of masculinity no longer works for male spectatorship either. 
8. 'The postwar era ... 1946 closed with three even bigger hits, The Jolson Story, Duel in the Sun, and The Best Years of Our Lives. These hits capped off what was by far Hollywood's biggest year ever in terms of box-office revenues and studio profits' (Schatz, 289-290).

9. 'In 1931 the film industry moved away from the central producer management system to a management organization in which a group of men supervised six to eight films per year, usually each producer concentrating on a particular type of film. Like other changes in the mode, this introduced greater specialization, in this case in the upper-management levels. In fact, over the period of the central producer system, specialization in the producer function was already occurring' (Bordwell, 320).

10. 'When Marty and I embarked upon this project, we never expected it to be so emotional. Originally, the format agreed upon was two 52-minute programs. However, as soon as we started charting our vast subject, conventional standards fell away. We had to create our own parameters, without fear of being selective or subjective' (Wilson, 8).

11. 'This trailer [for the animation film Shark Tale] showcases... a very entertaining exchange between De Niro and Martin Scorsese as Sykes, a puffer fish with the instantly recognizable Scorsese bushy eyebrows' (The Codfather).

12. See Rotten Tomatoes' General Discussion: 'Scorsese's eyebrows vs. Tarantino's forehead'.

13. 'Hitchcock's first job in the film industry was as a title illustrator for the British production branch of the most powerful of American companies - Paramount Pictures' (Ryall, 85).

14. '[I]t was for Alfred Hitchcock in the early Sixties - in Vertigo, North by Northwest and Psycho - that Bass evolved his most exalted, sophisticated and altogether disturbing form of abstraction' (Romney). 15. Something that relates to another aspect of The Interpretation of Dreams: 'Titles and credits thus consolidate an a priori impression in which the final sequence prevails over the first: the story will advance in a rectilinear fashion. They establish what Freud would call a "purposive idea", working beneath the development of the fiction as a structuring absence' (Vernet, 5).

16. ' $[D]$ uring the thirties each studio... typically developed a distinctive house style when it produced the most important films on its roster at the level where differentiation would normally be most effective.... The famous MGM look was created mainly by one individual, Cedric Gibbons. "The nearest thing to a movie star that Hollywood art direction ever had", Gibbons was "one of the most powerful personalities in America's most powerful studio for thirty years"' (Balio, 87). His appearance in MGM title sequences of that time was guaranteed: 'Joining the studio as head of the art department in 1924, he had a clause inserted in his contract stipulating that his credit would appear on every picture the studio produced, a stipulation that the studio respected with few exceptions until his retirement in 1956' (Balio, 87).

\section{Works Cited}

Allison, Deborah. 'Promises in the Dark: Opening Title Sequences in American Feature Films of the Sound Period.’ Ph.D. Diss. University of East Anglia, 2001.

Balio, Tino. Grand Design: Hollywood As a Modern Business Enterprise 1930-1939. Berkeley, Los Angeles, and London: University of California Press, 1993.

Bordwell, David, Janet Staiger and Kristin Thompson. The Classical Hollywood Cinema: Film Style \& Mode of Production to 1960. New York: Columbia University Press, 1985.

Bordwell, David. 'Sarris and the Search for Style', in: Emanuel Levy (ed.), Citizen Sarris, American Film Critic: Essays in Honor of Andrew Sarris, 165-173. Lanham and London: The Scarecrow Press, 2001.

- 'The Codfather'. Empire online. 17 May 2004. Available at: <http://www.empireonline. co.uk/site/news/NewsStory.asp?news_id=15850>. 
Durgnat, Raymond, and Scott Simmon. King Vidor, American. Berkeley, Los Angeles, and London: University of California Press, 1988.

Freud, Sigmund. The Interpretation of Dreams. Transl. and ed. by James Strachey. New York: Avon Books, 1965.

Gardies, André. 'Génèse, générique, générateurs ou la naissance d'une fiction'. Revue d'Esthétique 4, 86-120, 1976.

Harrington, Ralph. 'The Railway Accident: Trains, Trauma, and Technological Crises in Nineteenth-Century Britain', in: Mark S. Micale and Paul Lerner (eds.), Traumatic Pasts: History, Psychiatry, and Trauma in the Modern Age, 1870-1930, 30-56. Cambridge, and New York: Cambridge University Press, 2001.

Haskin, Pamela. “ "Saul, can you make me a title?” - Interview with Saul Bass'. Film Quarterly vol. 50 no. 1, 10-17, 1996.

Higham, Charles, and Joel Greenberg. The Celluloid Muse. New York: Signet, 1969.

Kernan, Lisa. Coming Attractions: Reading American Movie Trailers. Austin: University of Texas Press, 2004.

Kirby, Lynne. Parallel Tracks: The Railroad and Silent Cinema. Exeter: University of Exeter Press, 1997.

Kittler, Friedrich A. Discourse Networks: 1800/1900. Trans. by Michael Mettner, with Chris Cullens. Stanford: Stanford University Press, 1990.

Manovich, Lev. The Language of New Media. Cambridge and London: Miт Press, 2001.

Mulvey, Laura. 'Afterthoughts inspired by Duel in the Sun', in: Visual and Other Pleasures, 29-38. Houndsmills, Basingstoke, Hampshire, and London: Macmillan, 1989.

Romney, Jonathan. 'Let it roll! He wrecked "popcorn time" and turned the humble credit sequence into a film-goer's fetish: All hail Saul Bass, the Matisse of the movies...'. The Independent. July 18, 2004. Available at: <http://enjoyment.independent.co.uk/ low_res/story.jsp? story $=542645 \&$ host $=5 \& \operatorname{dir}=21>$.

Rotten Tomatoes, General Discussion: 'Scorsese's eyebrows vs. Tarantino's forehead', March 3, 2004. Available at: <http://www.rottentomatoes.com/vine/showthread. php?t=300692>.

Ryall, Tom. Alfred Hitchcock and the British Cinema. London and Atlantic Highlands, NJ: Athlone Press, 1996.

Sarris, Andrew. 'Notes on the Auteur Theory in 1962', in: Gerald Mast and Marshall Cohen (eds), Film Theory and Criticism, 650-665. New York and Oxford: Oxford University Press, 1979.

Schatz, Thomas. 'Boom and Bust: American Cinema in the 1940s. Berkeley, Los Angeles, and London: The University of California Press, 1997.

Scorsese, Martin. 'Duel in the Sun', in: The American Filminstitute (ed.). Private Screenings: Insiders Share a Century of Great Movie Moments. Atlanta: Turner Publishing, 140-143, 1995.

- 'Saul Bass as a Designer of Films'. The Masters Series: Saul Bass. March 11 to April 5, 1996. New York: Visual Arts Museum at the School of Visual Arts, 3, 1996.

- 'The Fundamental Film Teacher', in: Emanuel Levy (ed.), Citizen Sarris, American Film Critic: Essays in Honor of Andrew Sarris. vii, Lanham and London: Scarecrow Press, 2001.

Scorsese, Martin, and Michael Henry Wilson. A Personal Journey With Martin Scorsese Through American Movies. London: Faber and Faber, 1997.

Thompson, David, and Ian Christie (eds.). Scorsese on Scorsese. London and Boston: Faber and Faber, 1996.

Vernet, Marc. 'The Filmic Transaction: On the Openings of Film Noirs', in: The Velvet Light Trap 20 (Summer 1983): 2-9.

Wilson, Michael Henry. 'Preface' in: A Personal Journey with Martin Scorsese Through American Movies. 8-9, London: Faber and Faber, 1997.

Films Cited

Duel in the Sun. Dir. King Vidor. Selznick Pictures Corporation, usa, 1946.

Inklings: issue 12. Dir. Dave Fleischer. Inkwell Studios/Red Seal Pictures Corp., USA, 1925.

A Personal Journey With Martin Scorsese Through American Movies. Dir. Martin Scorsese and Michael Henry Wilson. British Film Institute / Miramax, GB/U SA, 1994. 



\section{Section Three}

Handwriting

and<smiles></smiles>

Embodiment 



\section{Writing on Archiving hachines}

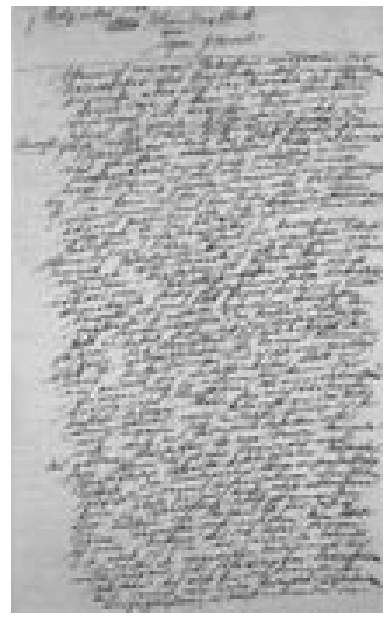

1. Freud's Notiz über den

'Wunderblock', 1924

\section{Exergue}

A paper on archiving machines has to start with a Freudian impression - the subtitle of Jacques Derrida's Archive Fever. ${ }^{I}$ Derrida distinguishes three forms of impression. The first Freudian impression is an inscription (Niederschrift), such as Freud's own Notiz über den 'Wunderblock', handwritten in 1924, printed in 1925, and translated in 1940 as 'A Note upon the "Mystic Writing Pad"', reactivated in 2000 by artist Arnold Dreyblatt in his installation 'The Wunderblock' (Freud quoted in Draaisma, 7-9; Dreyblatt).

This first Freudian impression evokes a second one. With his Note, Freud made an impression - he continues to make an impression - 'on anyone, after him, who speaks of him or speaks to him', among them Derrida who carried Freud's discussion on the mystic pad further. Arnold Dreyblatt, too, showed with his Wunderblock and other installations how he is impressed by Freud, while at the same time making new Freudian impressions. 
A third meaning of impression is the undetermined notion. 'Archive' is such an impression: 'only a notion, an impression associated with a word and for which together with Freud, we do not have a concept. We only have an impression...' (Derrida 1996, 29). It is this third form of impression, in conjunction with the first one, the scriptural impression, that is the subject of my paper.

\section{Pleamble}

According to Freud, memory traces (Erinnerungsspuren) of perceptions are not stored permanently in the perceptual system, but in memory systems underneath. He demonstrated this with the mystic writing pad. This is a child's toy, consisting of a pad covered with wax, protected by two sheets, one of wax paper and another of celluloid. Writing with a stylus on the celluloid sheet leaves traces on the wax paper, visible through the celluloid. The text can be erased by pulling the paper loose from the wax layer. The celluloid sheet corresponds with our perception of consciousness that observes the stimulus, but does not preserve it. The permanent traces (Dauerspuren) of the stimulus/stylus are apprehended in the wax layer of the mystic pad, analogous to the unconscious mnemic system behind the perception-consciousness. Writing on the mystic pad, as described by Freud, does not depend on material being deposited durably upon the receptive surface: the writing leaves permanent traces in/on a mnemic system which is invisible to the observer.

From the human mnemic system, pulses are sent to the porous perceptive layer - to and fro. But it is precisely this capacity - reproducing the once-faded writing from within - that the mystic pad is lacking. Consequently, the mystic pad is not a true archiving machine which both stores and retrieves and (re)produces memorytraces.

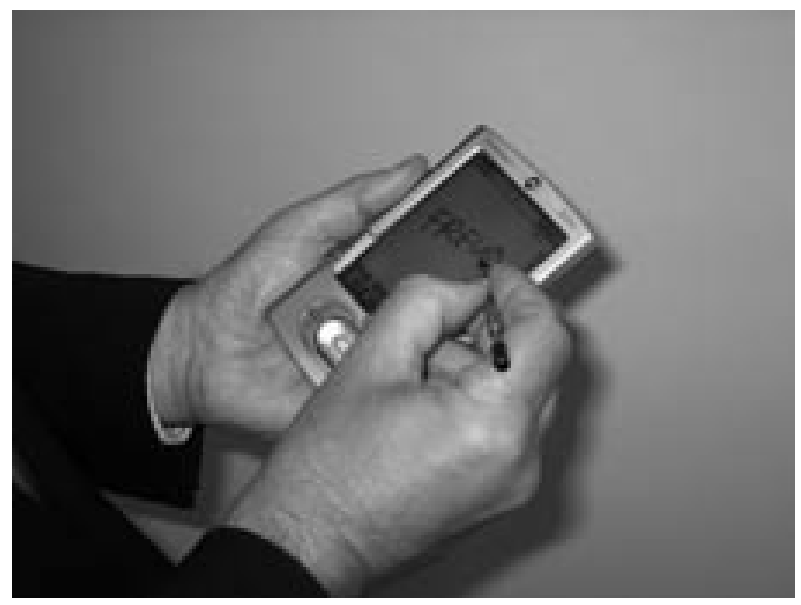


The modern mystic writing pad - and a true archiving machine - is a keyboardless tablet PC or PDA. We write with a stylus on its surface (the screen). The writing leaves no mark on the substrate; it looks - to use the poetic expression that the Flemish philosopher Ann van Sevenant adopted from Plato - like writing with water (Van Sevenant). The stylus does not penetrate 'the virginity of the receiving substance' (Derrida 1978, 251).

Can we still call such writing on a PDA handwriting? Was it our hand? To what extent is the hand that held the stylus identifiable? Writing with a stylus on a tablet PC or PDA produces, in some applications, handwriting just as if the screen were a piece of paper marked by a pen or a pencil. Such handwriting can subsequently be reproduced from the machine's memory, but only because the impressions on the screen have been translated by software into bits, which the machine can 'read', store, retrieve and re-translate into handwriting. The immediacy of writing on a screen is an illusion, as is its corporeality. Does this render the tablet PC and the PDA useless as a modern mystic writing pad, as prosthesis of live memory?

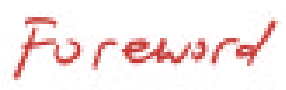

The issue here is not so much that digital writing and storage are metaphors for human memory, but that (hand)writing can be seen as a means of archiving. Derrida's main question with regard to memory is how the psychic apparatus is:

affected differently by all the technical mechanisms for archivization and for reproduction, for prostheses of so-called live memory, for simulacrums of living things which already are, and will increasingly be more refined, complicated, powerful than the 'mystic pad' (microcomputing, electronization, computerization, etc.)? (Derrida 1996, 15)

The metaphor of writing haunts European discourse (Derrida 1978, 197). Writing, Schreiben, schrijven are ambiguous words, referring to both the process and the product. The process is defined in the Concise Oxford Dictionary as marking 'paper or some other surface by means of a pen, pencil, etc. with symbols, letters, or words.' The product of such writing is 'that which is in a written state or form', 'a group or sequence of letters or symbols'. ${ }^{2}$

In German and Dutch the double meaning is even stronger: man schreibt ein Schreiben, men schrifft een schrijven: one writes a writ (a written formal document). The German and Dutch verb and noun schreiben, schrijven come from the Latin scribere (as do the English scribe and scrivener). ${ }^{3}$

The distinction between writing as a process and writing as a product has an interesting parallel in the distinction between archive as a process and archive as a product. The archive-product, as we will see, is reconstructible by means of the archive process. Before we can assess the extent to which handwriting is equivalent to writing on, in, or with archiving machines, we have to understand the recovering features of the archive process. 


\section{Theres}

\section{The Archive}

What is the archive? Is it 'only a notion, an impression'? Whereas most English and French archivists always use the plural 'archives', scholars outside the archival profession have started to use the singular: the archive. For most anthropologists, sociologists, philosophers, cultural and literary theorists the archive is the Foucauldian archive: 'the general system of the formation and transformation of statements' (Foucault, 145-146).

In archivists' terminology, in German and Dutch, het archief/das Archiv (singular) is used both for the institution and the building that houses the archives (plural). This corresponds with the origin of the word archive, coming from the Greek archeion: 'the residence of the magistrates, the archons, the place where official documents are filed' (Derrida 1996, 2).

Today's archeion is an archive(s) like the National Archives, an institution that manages and preserves archives. Archives, the totality of documents, are defined in archival terminology as recorded information, regardless of form or medium, created, received, and maintained as evidence by an agency, institution, organization or individual in pursuance of legal obligations or in the transaction of business (Walne). The qualification is important 'regardless of form or medium', indicating that neither the mode of inscription (handwritten or typewritten, painted or printed) nor the carrier (clay tablet, parchment, paper) are essential. The archival bond is essential, which binds the documents to the context of their creation.

\section{Dynamic Archiving}

Archivists and historians use archives as sources, yielding documentary evidence about the past. Moreover, good archivists and historians

have always studied not just what is said, but how the message is expressed: the language, the medium, the technology of production, the genre of the document, the historical circumstances and the context of writing (who the author is, who the intended audience is). (Heald, 93)

Disciplines other than history have also discovered the archives. Anthropologist Ann Stoler explains that the 'archival turn' in anthropology is leading her and her colleagues to critically reflect on the making of documents and how we choose to use them, on archives not as sites of knowledge retrieval but knowledge production, as monuments of states as well as sites of state ethnography. This is not a rejection of colonial archives as sources of the past. Rather, it signals a more sustained engagement with those archives as cultural artifacts of fact production, of taxonomies in the making, and of disparate notions of what made up colonial authority. (Stoler 'Colonial Archives and the Arts of Governance', 90-91; Stoler 'On the Content in the Form', 85)4 
Scholars in other disciplines too, are looking $u p$ from the archival document and through the archive, looking beyond - and questioning - its boundaries (Blouin and Rosenberg). In doing so, the focus shifts from the actual archival document to its functional process or context of creation (Yakel); from the archive as a product to archive as a process; from the physical artifact to the 'very act and deed' which first caused that artifact to be created (Taylor); shifting the emphasis from the analysis of the properties and characteristics of individual documents to an analysis of the functions, processes, and transactions which cause documents to be created (Cook, 47).

In this new approach, an archive and archiving are much more than the storage of a document (Webster's: 'to file or collect as records or documents in or as if in archive'). Archiving encompasses all the activities from the capture of documents into the system, to their management, use, and disposal. Archiving implies interactions, interventions, interrogations, and interpretations by creator, user, and archivist; these are activations that co-determine the archive's meaning (Ketelaar 2001).

This implies that the archive is not static, but a dynamic process (Ernst 2002, 138-139). Quite appropriately, a recent German collection of essays is entitled Archivprozesse. Die Kommunikation der Aufbewahrung [Archival processes. The communication of storage]. Jürgen Fohrmann, in the collection introduction, explains that the archive should be seen as a dynamic process:

If everything that originates from the archive, is modeled by the work of the user, then again put into the archive, to be activated over again etc. then the archive is not only to be understood as thesaurus, as place, as Wunderkammer, but as process. (Fohrmann, 22)

The archive product is also formed by the archive process because any use of the archive retrospectively affects all earlier instances of use, or, to put it differently: we can no longer read a text as our predecessors have read it (Ketelaar 2001). Let me give an example. The records created and used by German and Dutch agencies during World War II to account for the looting of Jewish assets, continued to be used, after the war, by German and Dutch agencies in the processes of restitution and reparation. The same record was activated again and again by different societal powers, for different purposes and for different audiences, as it continues to be activated today in the search for looted and lost works of art and other Holocaust assets. The looting and the registration of the looted property were, of course, an appalling event, but it was through the subsequent reemployment of the record that the primary registration really became a record of a traumatic experience. This is an application of Freud's Nachträglichkeit (retrospective causality): events that occur later may not only change the significance, but the nature of prior events as well (Van Zyl, 53-55). Archives do not have 'a single past, but an unbroken sequence of past times leading backward from the present moment.'5 
Archives, however, are not only leading backward: they secure the present for the future, transmitting authentic evidence of human activity and experience through time (Ernst 2002, 120-122). Archives, libraries, museums are all in the 'memory business', ensuring 'time future contained in time past' (Ketelaar 2004). But what distinguishes archives from all other memory institutions is that the individual, organizational, and collective memories they preserve are not defined primarily in terms of a cultural heritage because they have been created as 'process-bound information': '[I]nformation generated by coherent work processes and structured and recorded by these work processes in such a way that it can be retrieved from the context of those work processes' (Thomassen, 374).

Archives are memory because they are evidence. They are not only evidence of a transaction, but also evidence of some historic fact that is either part of the transaction itself, or that may be traced via the transaction, or that which is otherwise embodied in the record, or in the context of the archiving process.

\section{Archivalization}

Archiving is mostly understood to be the activity that follows upon the creation of a document. Archival theory, however, carries archiving one step forward because at the front end of a record-keeping system, documents are captured, that is, accepted by the system. Derrida has introduced the notion of archivation (archivization in the English translation). ${ }^{6}$ Archivization extends beyond capture, it includes the creative phase before capture, it is consigning, inscribing a trace in some external location, some space outside. Paul Ricoeur uses the term archivation too, referring to writing down the oral testimony and then setting aside, assembling, and collecting these traces (Ricoeur, 166-168).

Before archivization, however, before the impression of a document, there is a 'moment of truth' (Stuckey, 270) which I propose to call archivalization, a neologism, meaning the conscious or unconscious choice (determined by social and cultural factors) to consider something worth archiving (Ketelaar 1999; 2000, 328329). Archivalization precedes Derridean archivization and archiving. The searchlight of archivalization has to sweep the world for something to illuminate in the archival sense, before we proceed to register, to record, to inscribe it, in short, before we start archivization and archiving.

We are looking at the Pompeii fresco of a girl writing (Illustration 3). Is she writing? Is she thinking? About what? I would suggest that we are looking at someone in the process of archivalization, pondering her choices of whether to inscribe her thoughts or not, whether to take the next step or not, toward archivization - handwriting the trace and then to archive it.

By differentiating archivalization from subsequent inscription or archivization, which is then followed by archiving, we gain a better understanding of the tacit narratives of the archives (Ketelaar 2001). These tacit narratives, the meanings of archives, must be inferred from the content, structure, and form of the archival documents. 


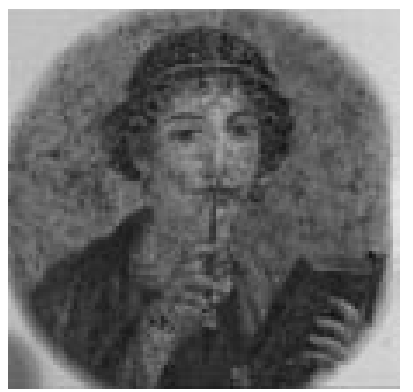

3. The process of archivalization

\section{Digital Records}

In paper records, content, structure, and form are physically present in the document and its physical arrangement. Digital records, however, do not present their content, structure, and form in or on a physical medium, but rather in a digital representation that serves as a generator for various ways in which the document can be made visible (Simons, 57). Digital documents are potential documents, that emerge only by virtue of software that understands how to access and display them (Rothenberg). Moreover, the circumscription of a digital document is different from a physical document because it often includes links to other documents, and it is variable and changeable, fluid and unstable. ${ }^{7}$ An original no longer exists because, intrinsically, each recording or representation (on a medium, a screen or as a printout) is a representation or rather a reconstruction made by the operating system and the application software. ${ }^{8}$ As David Levy notes, digital materials are made up of both the digital representation and the perceptible forms produced from it. The digital archive can only be communicated through space and time by being continuously 'manufactured just-in-time, on the spot' (Levy, 152). 'Ensuring the technical and intellectual survival of authentic records through time', as ISO standard 15489 requires, means reconstructing the content, form, and structure of a record through time, every time making an 'authentic copy' of an original that never existed in reality but only as a virtual construction.

The original in the digital age has disappeared, it has to be reconstructed time and again by means of copies: the original is inscribed in its copy (Ernst 1988, 515). The copy permits a (re)construction of the original. Retrieving the original by reconstruction is like the reconstruction of information stored in human memory. As neurologist Antonio Damasio explains, the memory you have of someone you met is not stored as a lifelike copy, not like a Polaroid picture, but rather as 'dispositions', a set of codings for reactivation or reconstruction:

And once you apply the reconstruction mechanism, you have a chance of bringing to your mind some kind of image that approximates the image that you actually had in perception. (Damasio and Mulder 2003) 
The 'disappearance of the original' in the digital age has led to a major paradigm shift in archival science, as Hugh Taylor foresaw in as early as 1988 (Taylor). Archival science can no longer utilize many of the concepts that were applicable in the world of paper and parchment. ${ }^{9}$ Its object is no longer a tangible document or file in a logical and partly physical context that can be arranged and described, used and preserved, as in the paper world: the object is the archive process rather than the archive product.

\section{Duplex Writing/Reading}

A digital document is not a thing in and of itself. It is 'no more than an interpretive moment in a never-ending conversation with the texts'. ${ }^{\text {IO }}$ The digital document speaks in a dialogue with the reader/viewer, who may be outside the visible text, but never outside its invisible narrative. ${ }^{I I}$ In our digital world, as Mark Poster affirms 'Texts become "hypertexts" which are reconstructed in the act of reading, rendering the reader an author' (Poster, 188). The text becomes an interactive dialogue between 'the writer and her readers, and the reader could be asked to share the responsibility for the outcome' (Bolter, 112). Writing becomes part of a dual process of writing/reading. The writer and the reader sit at their keyboardless computer screens and connect with their archiving machines via a stylus or their voices or the heat of their fingers as they touch the screen. The hand of handwriting has been transformed into a part of the machine, entailing not only 'a profound transformation of the body and of our relationship to our own body' (Derrida and Stiegler, 96), but also embodying handwriting with a new functionality.

\section{Digital Handwriting}

The hand that is writing on a keyboardless tablet PC or PDA connects the human body with a machine body, leaving no permanent mark on the substrate, but transferring from one body to another the potentiality of reconstruction. Contingent to that reconstructibility are the features of conventional handwriting: immediacy, singularity, iterability and corporeality - or rather the digital equivalents of these features. Immediacy and corporeality of digital handwriting are an illusion. The archiving machine stores a simulacrum of what has been 'written' (i.e., constructed by the hand interfacing with the machine), it may even be able to 'remember' (i.e., reconstruct) the writing hand. Moreover, even the writing mind can be reconstructed by means of an archaeology of the mise-en-abime of continuous archivalization and archivization. From the archival machine behind the screen we can recall a 'copy' of what we wrote. The copy permits a (re)construction of the original. To quote Aleida and Jan Assmann refuting Walter Benjamin, the original is not displaced by its copies, but is constructed by them and can thrive with them' (Assmann and Assmann, 156). Similarly, handwriting as a product may be displaced by writing on archiving machines, but handwriting as a process can be constructed through the archiving process and can thrive. 


\section{Archival Constructs}

This 'new' writing (hands-on or hands-off) on archiving machines constitutes one of what Derrida calls archival technologies that determine 'not merely the moment of the conservational recording, but rather the very institution of the archivable event' (Derrida 1996, 18). Archives do not merely serve to preserve for the future an archivable content of the present. Rather, that present and its relation to the future are constructed through archiving: in Derrida's words 'The archivization produces as much as it records the event' (Derrida Archive Fever, 17; 'Archive Fever. A Seminar', 35). The Amsterdam Achterhuis (the Secret Annex) - I do not mean the brick and mortar one, but the Achterhuis as a lieu de mémoire - exists only because of its archivization in Anne Frank's Diary. Another example: according to Dutch law, the fact of a child's birth only exists by virtue of its registration. Therefore, when a baby dies before its birth has been registered, a birth certificate is not issued, only a certificate stating that the child was not alive at the moment of declaration. In other words, the birth has never happened, because it was never registered (Ketelaar 1999, 57). A photograph or a video is not just a recording: it constitutes the event. Think of home movies: the happy moments in the movie are staged, they are produced by their archivization in the movie (Roepke). Even what is transmitted on television 'live' is 'produced before being transmitted' (Derrida and Stiegler, 40).

Archivization systems are active creators of categories because people in these systems, through archivalization, 'classify away traces that they know to be relevant but which should not be officially recorded' (Bowker and Star, 279, 321). It is impossible to die of old age, because the International Classification of Diseases does not recognize 'being worn out' as a cause of death (Bowker and Star, 90, 276). Bowker and Star give many other examples of the way in which life is constructed and ordered by classification, labeling and categorization. Their study affirms Derrida's assumption that 'the mutation in technology changes not simply the archiving process, but what is archivable - that is, the content of what has to be archived is changed by the technology' (Derrida Archive Fever 6, 17; 'Archive Fever: A Seminar', 46; Ketelaar 1999, 2000). Technology conditions not only the form or the structure, but also the content of the writing. Of course, technology is not the only factor that determines form and content, because cognitive and cultural agencies also play an important role, as demonstrated by the following examples.

Until quite recently, Dutch people were instructed to deal with only one subject when writing a letter to the government - the governmental filing system, with one file for each subject, could not cope with a citizen's letter that included more than one subject (Ketelaar 1997, 214-215). ${ }^{\text {I2 }}$ The technology of archivization conditions the archivalization (the choice of a citizen to deal with a particular topic in her letter), which in turn, conditions archivization and archiving because when incoming letters deal with only one subject, they can be dealt with in a relatively simple work process, sustained by a relatively simple document management system.

When one uses Sms (Short Message Service) on a mobile telephone, the form and content of the message will be different from a message sent by e-mail or a 
message conveyed in a handwritten letter. This difference is due to the technology in terms of a complex interplay with social and cultural norms governing what to SMS and how to SMs. ${ }^{\text {I3 }}$

Likewise, the technology of handwriting on a tablet PC or PDA conditions the content of the writing, which to a large extent will be different from the content of a handwritten text on paper.

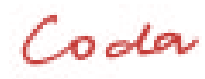

In the preceding sections I have argued that the archive-as-a-process works in two ways. Looking backward - before the impression or archivization - we encounter archivalization. And looking the other way, we see that the archive is never closed, it is shaped by the anticipation of the future - the future anterior (Derrida Archive Fever, 18, 68; 'Archive Fever: A Seminar', 40). But this power of the archive is limited by the paradox of anticipation: the archive fever which, by anticipating death, is already 'deadening death [à amortir la mort] to the point where it can't even happen anymore' (Derrida and Stiegler, 106). Here and now, the 'impression' produces as much as it records the event, embodying the event with meaning, while staying receptive to future activations of the archive which will add to and change its former meanings: 'a perpetual endgame of inscriptions' (Brothman, 192).

The handwriting on, in or with an archiving machine that is kept in the machine's archive memory is only decipherable and understandable in terms of the processes of archivalization, archivization and archiving through which the archive product was constituted and subsequently activated and re-activated by ever new impressions.

It does not matter by what technology these impressions are inscribed, as long as the archiving machine keeps a trace of what has been traced on the surface.

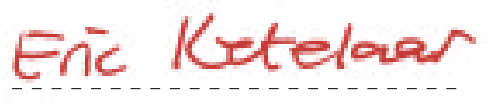

SIGN HERE! 
Notes

1. Derrida's translator Eric Prenowitz writes: 'Archival machines. I prefer archiving machines as a translation of machines à archiver. Reversely, the machines à archives in Derrida's L'Écriture et la différence (translated in Derrida's Writing and Difference as 'machines for storing archives') might be called archival machines (Archivmaschinen).

2. According to the Oxford English Dictionary: 'The action of one who writes, in various senses; the penning or forming of letters or words; the using of written characters for purposes of record, transmission of ideas, etc.' 'That which is in a written (now also typewritten) state or form; something penned or recorded; written information, composition, or production; literary work or compilation.'

3. The word was adapted at a time when the Germans adopted the Roman way of writing, replacing the scratching of runes (krassen, German: reissen, old Dutch rijten, compare writing). See N. van Wijk, Franck's Etymologisch woordenboek der Nederlandsche taal, 2nd ed. 's-Gravenhage: Martinus Nijhoff, 1912.

4. See also Betty Joseph, Reading the East India Company, 1720-1840: Colonial Currencies of Gender, Chicago: University of Chicago Press, 2004.

5. See Kenneth L. Ames, 'Introduction', in: Alan Axelrod (ed.), The Colonial Revival in America, Henry Francis du Pont Winterthur Museum, Wilmington, DE, 1975, 6, quoted by Spencer R. Crews and James E. Smis in 'Locating Authenticity: Fragments of a Dialogue', in: Ivan Karp and Steven D. Lavine (eds.), The Poetics and Politics of Museum Display, 160, Washington and London: Smithsonian Institution Press, 1991.
6. The term archivation was first used in the nineties by the French philosopher Bernard Stiegler, see Chabin, 66. French archivists, however, used the term before as the equivalent of archivéconomie or archivage, Delmas 2001, 28.

7. See John Mackenzie Owen, 'Authenticity and Objectivity in Scientific Communication: Implications of Digital Media' in this volume, p.60.

8. In modern diplomatics, an original is 'the first complete and effective record', see Duranti; MacNeil; and Duranti, Eastwood and MacNeil. Following Delmas 1996 , I contend that every (digitally created) view (or instantation) of a (digitally created) 'original' is a reconstruction. 9. In other disciplines, the concept of an 'original' is also challenged: 'Most medieval scholars would be hard pressed to correlate the term "original" with any given manuscript... Text-editors would reject outof-hand the notion of "original" applied to a specific manuscript...' (Nichols, 264). 10. See Dodge, 19. Also see Cook 2001 and Ketelaar 2001, 10.

11. See Harris 1997, 136; Harris 2000, 20; Harris 2002, 65.

12. 'The limits inherent in writing and typing on paper to some extent dictate the kind of policy which can be effectively pursued... the early indexed filing systems allowed for the solution of limited, reactive problems but did not facilitate long-term planning based on coordinated information' (Taylor).

13. Young people tend to use 'SMS language' not only in writing an SMS but in e-mail and letters as well - an interesting form of remediation of a cultural practice.

\section{Works Cited}

Assmann, Aleida, and Jan Assmann. 'Air from other Planets Blowing: The Logic of Authenticity and the prophet of the Aura', in: Hans Ulrich Gumbrecht and Michael Marrinan (eds.), Mapping Benjamin: The Work of Art in the Digital Age, 147-157. Stanford: Stanford University Press, 2003.

Blouin, Francis X., and William G. Rosenberg (eds.). Archives, Documentation, and Institutions of Social Memory: Essays from the Sawyer Seminar. Ann Arbor: University of Michigan Press, 2005.

Bolter, J. David. Writing Space: Computers, Hypertext, and the Remediation of Print. 2nd ed. Mahwah, NJ: Lawrence Erlbaum Associates, 2001.

Bowker, Geoffrey C., and Susan Leigh Star. Sorting Things Out: Classification and Its Consequences. Cambridge: MIT Press, 1999. 
Brothman, Brien. '[Review of] “Jacques Derrida, Archive Fever”, in: Archivaria 43, 191-92, 1996.

Chabin, Marie-Anne. Je pense donc j'archive. Paris and Montréal: L'Harmattan, 1999.

Cook, Terry. 'What is Past is Prologue: A History of Archival Ideas since 1898, and the Future Paradigm Shift'. In: Archivaria 43, 17-63, 1997.

Damasio, Antonio, and Arjen Mulder. 'The Memory as Living Archive. Interview with Antonio Damasio', in: J. Brouwer, A. Mulder, and S. Charlton (eds.), Information Is Alive: Art and Theory on Archiving and Retrieving Data. Rotterdam: v2 Publishing/ NAIPublishers, 2003.

Delmas, Bruno. 'Manifesto for a Contemporary Diplomatics: From Institutional Documents to Organic Information', in: American Archivist 59, 438-452, 1996.

- 'Archival Science Facing the Information Society',in: Archival Science 1, 25-37, 2001.

Derrida, Jacques. Archive Fever. Transl. Eric Prenowitz. Chicago and London: University of Chicago Press, 1996.

- 'Archive Fever. A Seminar...', in: Carolyn Hamilton et al. (eds.), Refiguring the Archive, 38-80. Dordrecht, Boston, and London: Kluwer Academic Publishers, 2002.

- Writing and Difference. Transl. Alan Bass. London and New York: Routledge, 1978.

Derrida, Jacques, and Bernard Stiegler. Echographies of Television: Filmed Interviews. Transl. Jennifer Bajorek. Cambridge: Polity Press, 2002.

Dodge, Bernadine. 'Across the Great Divide: Archival Discourse and the (Re)Presentation of the Past in Late-Modern Society', in: Archivaria 53, 16-30, 2002.

Draaisma, Douwe. Metaphors of Memory: A History of Ideas about the Mind. Cambridge and New York: Cambridge University Press, 2000.

Dreyblatt, Arnold. Aus den Archiven / From the Archives. Heidelberg: Stadtgalerie Saarbrücken and Kehrer Verlag, 2003.

Duranti, Luciana. Diplomatics: New Uses for an Old Science. Lanham, MD and London: Society of American Archivists and Association of Canadian Archivists in association with Scarecrow Press, 1998.

Duranti, Luciana, Terence M. Eastwood, and Heather MacNeil. Preservation of the Integrity of Electronic Records. Dordrecht/Boston/London: Kluwer Academic Publishers, 2002.

Ernst, Wolfgang. ' (in)Differenz: Zur Extase der Originalität im Zeitalter der Fotokopie', in: Hans U. Gumbrecht and Karl L. Pfeiffer (eds.), Materialität der Kommunikation. 498-518. Frankfurt: Suhrkamp, 1988.

- Das Rumoren der Archive. Ordnung aus Unordnung. Berlin: Merve Verlag, 2002.

Fohrmann, Jürgen. “"Archivprozesse” oder über den Umgang mit der Erforschung von “Archiv”. Einleitung', in: Leander Scholz and Hedwig Pompe (eds.), Archivprozesse: die Kommunikation der Aufbewahrung, 19-23. Köln: DuMont, 2002.

Foucault, Michel. The Archaeology of Knowledge. Transl. A.M. Sheridan Smith. London and New York: Routledge, 2002.

Freud, Sigmund. 'Notiz über den "Wunderblock", in: Gesammelte Werke, 3-8. Frankfurt am Main: Fischer Verlag, 1948.

Harris, Verne. 'Claiming Less, Delivering More: A Critique of Positivist Formulations on Archives in South Africa', in: Archivaria 44, 132-141, 1997.

- Exploring Archives: An Introduction to Archival Ideas and Practice in South Africa. 2nd ed. Pretoria: National Archives of South Africa, 2000.

- 'A Shaft of Darkness: Derrida in the Archive', in: Carolyn Hamilton et al. (eds.). Refiguring the Archive. Dordrecht, Boston, and London: Kluwer Academic Publishers, 61-81, 2002.

Heald, Carolyn. 'Is There Room for Archives in the Postmodern World?', in: American Archivist 59, 88-101, 1996.

Ketelaar, Eric 'Recordkeeping Systems and Office Technology in Dutch Public Administration, 1823-1950', in: Jahrbuch für europäische Verwaltungsgeschichtel Yearbook of European Administrative History 9, 213-222, 1997.

- Archivalisation and Archiving', in: Archives and Manuscripts 27, no. 1, 54-61, 1999.

- 'Archivistics Research Saving the Profession', in: American Archivist 63, no. 2, 329-330, 2000. 
- 'Tacit Narratives: The Meanings of Archives', in: Archival Science 1, 143-155, 2001.

- 'Time Future Contained in Time Past: Archival Science in the 21st Century', in: Journal of the Japan Society for Archival Science 1, 20-35, 2004.

Levy, David M. Scrolling Forward: Making Sense of Documents in the Digital Age. New York: Arcade, 2001.

MacNeil, Heather. Trusting Records: Legal, Historical and Diplomatic Perspectives. Dordrecht/Boston/London: Kluwer Academic Publishers, 2000.

Nichols, Stephen G. 'The End of Aura?' In: Hans Ulrich Gumbrecht and Michael Marrinan (eds.), Mapping Benjamin: The Work of Art in the Digital Age, 256-268. Stanford: Stanford University Press, 2003.

Poster, Mark. What's the Matter with the Internet?. Minneapolis: University of Minnesota Press, 2001.

Ricoeur, Paul. Memory, History, Forgetting. Chicago: University of Chicago Press, 2004.

Roepke, Martina. 'Feiern im Ausnahmezustand. Ein privater Film aus dem Luftschutzkeller'. In: montagelav 10 February 2001, 59-66, 2001.

Rothenberg, Jeff. Avoiding Technological Quicksand: Finding a Viable Technical Foundation for Digital Preservation. Washington DC: Council on Library and Information Resources, 1999.

Simons, Johannes A.A. Interface en Cyberspace. Inleiding in de Nieuwe Media. Amsterdam: Amsterdam University Press, 2002.

Stoler, Ann L. 'Colonial Archives and the Arts of Governance', in: Archival Science 2, 87-109, 2002.

- 'Colonial Archives and the Arts of Governance. On the Content in the Form', in: Carolyn Hamilton et al. (eds.), Refiguring the Archive, 83-100. Dordrecht, Boston, and London: Kluwer Academic Publishers, 2002.

Stuckey, Steve. 'Record Creating Events: Commentary', in: Archives and Museum Informatics 11, 269-270, 1997.

Taylor, Hugh. " "My Very Act and Deed": Some Reflections on the Role of Textual Records in the Conduct of Affairs', in: American Archivist 51, 456-469, 1988; reprinted in Cook, Terry, and Gordon Dodds (eds.), Imagining Archives: Essays and Reflections by High A. Taylor. Lanham and Oxford: Society of American Archivists and Association of Canadian Archivists in association with The Scarecrow Press, 2003.

Thomassen, Theo. 'A First Introduction to Archival Science', in: Archival Science 1, 373-385, 2001.

Van Sevenant, Ann. Met water schrijven. De filosofie in het computertijdperk. Antwerp and Baarn: Hadewijch, 1997.

Van Zyl, Susan. 'Psychoanalysis and the Archive: Derrida's Archive Fever', in: Carolyn Hamilton et al. (eds.), Refiguring the Archive. Dordrecht, Boston, and London: Kluwer Academic Publishers, 39-59, 2002.

Walne, Peter. Dictionary of Archival Terminology: English and French, with Equivalents in Dutch, German, Italian, Russian, and Spanish. 2nd rev. ed. Munich and New York: K.G. Saur, 1988.

Yakel, Elizabeth. 'The Way Things Work: Procedures, Processes, and Institutional Records', in: American Archivist 59, 454-464, 1996. 


\section{Blood Samples and Fingerpint Files Blood as Artificial Matter, Artistic Material, and Means of the Signature}

\section{mbrotuction}

All handwriting leaves a trace, even if it is only the trace of an absent body that produced this trace. This trace can be anything from a drawn line or a scribble to a short note, a sketch or a signature, and it can mean anything, ranging from mere 'non-sense' produced in the process of a bodily automatism while thinking of something else, to a meaningful act of leaving a message or even of authenticating oneself through the act of signing.

Therefore, writing, handwriting, and drawing may always commence with a playful act without definite purpose: a face drawn in the sand with one finger by a child and then signed by the first letters it is able to write - or able to imagine as her or his own name. From there on, the technique is ever improved, as are the tools extended where the hand learns to control its movements, and where one finger that left a trace in the sand is extended and replaced by the tool of a pencil or pen. The index (or thumb) which so often left an immediate stain on a piece of paper or textile, or a temporary impression on the skin of another person, begins to lose direct contact with the bearer of traces - be it paper, parchment or skin.

The connection that my text reflects on is the seemingly obvious one of (hand-) writing-signature and trace-impression/print, which also implies the dialectics of presence/absence of a body. That presence/absence may be less manifest when we 
regard the liquid normally used within the writing process but which immediately gains another (symbolic) quality when it undergoes a substitution of, for example, ink for blood.

My approach is based on Mieke Bal's and Norman Bryson's article 'Semiotics and Art History' and their reference to Jacques Derrida. According to Bal and Bryson the signature:

is the most typical case of the Derridian 'trace', the indexical sign that refers by contiguity, not simply to the past (the maker of the image) but, more importantly, to the future, the reading of it. The act of writing, which for Derrida is much more than graphic writing alone, is precisely the production of traces, and painting, drawing, and, most emphatically, etching are also acts of writing in this sense. (Bal and Bryson, 190)

When, therefore, considering the relation between the trace and the impression, or print, one also has to keep in mind the footstep, the fingerprint, the mark, the cast and - last but not least - the copy. Georges Didi-Huberman, in his essay on the impression ('L'empreinte'), uses the example of the footstep to clarify their relatedness: in order to produce a footstep, the foot has to sink into the sand, but one has to remove the foot in order to render the footstep, or its impression, visible. ${ }^{I}$ In other words, the impression (empreinte) is dependent upon a dialectics of presence and absence. A main condition, or requirement, is, of course, a form of contact, a touch. The remains of such a touch - the trace or print - is characterized by its 'indexicality'. But, as we will see, this indexicality turns into iconicity when the liquid used for handwriting is consciously changed or even intentionally supplemented.

Therefore, I will make a few theoretical remarks on the index and the icon before I start with a short overview of the use of blood in contemporary arts and then look a bit more closely at a work by Jenny Holzer.

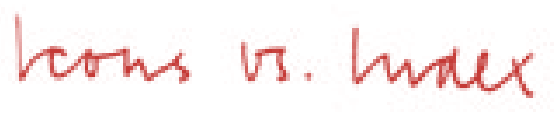

Charles Sanders Peirce, in his typology, defined the sign as tripartite, comprised of icon, index, and symbol. I shall confine myself to icon and index in this context. According to Peirce's definition:

an icon is a sign which would possess the character which renders it significant, even though its object had no existence; such as lead-pencil streak as representing a geometric line. An index is a sign which would, at once, lose the character which makes it a sign if its object were removed, but would not lose that character if there were no interpretant. Such, for example, is a piece of mould with a bullet hole in it as a sign of a shot; for without the shot there would have been no hole; but there is a hole there, whether anybody has the sense to attribute it to a shot or not. [A symbol is a sign which 
would lose the character which renders it a sign if there were no interpretant. Such is any utterance of speech which signifies what it does only by virtue of its being understood to have that signification.] (Peirce, quoted in Bal and Bryson, 189)

Mieke Bal and Norman Bryson, in their article 'Semiotics and Art History', do not only warn against identifying the icon with the entire domain of the visual (although I ignore this warning since I shall, as you will see, concentrate on the visual!). On the contrary, Bal and Bryson emphasize that the icon can be:

best seen as a sign capable of evoking nonexistent objects because it proposes to imagine an object similar to the sign itself. Iconicity is in the first place a mode of reading, based on a hypothetical similarity between sign and object. (Bal and Bryson, 189)

Bal and Bryson stress Peirce's emphasis on a symmetrical opposition of icon and index insofar as:

while the icon does not need the object to exist, the index functions precisely on the ground of that existence. His [Peirce's] example suggests that real, existential contiguity between indexical sign and object (or meaning) is indispensable. (Bal and Bryson, 190)

To put my conclusion in terms of a question: Can (or shall) we regard this symmetrical opposition between icon and index to be in any way analogous to the opposition that Walter Benjamin establishes in the notions of aura and trace ('Spur') in his 'The Work of Art in the Age of Mechanical Reproduction'? If the aura represents an absent presence and the trace a present absence, can this be said vice versa of the index and the icon?

The fingerprint (as pen) and the human skin (as writing-surface) will, in the following, serve as 'media' to further develop these questions.

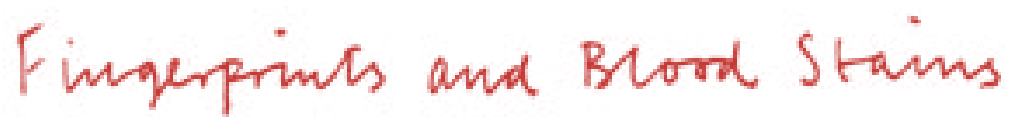

When Iraqi citizens, during the presidential elections in Iraq in the Summer of 2002, wanted to express their absolute loyalty to Saddam Hussein, they did so in a peculiar way. The voters pressed their (right) thumb, impregnated with their own blood, on their ballots. The gesture was certainly meant to emphasize the unconditional faithfulness of the voter: the fingerprint stood for, or replaced, the signature (or the voting ' $\mathrm{X}$ ') and the sentiment was confirmed by the substitution of the voter's own blood for normal ink. Because that 'very particular juice' ('ein ganz besonderer Saft', according to Mephistopheles in Faust) that is blood serves here as a means of reinforcing authenticity by placing emphasis on the fingerprint as abso- 
lutely authentic: both the blood and the fingerprint belong to a particular person and, moreover, are part of his body or at least an immediate body effect. Moreover, in direct relation to this reinforcement, the gesture made with the bloodsoaked finger expresses a certain readiness, namely the readiness to die as a martyr.

For the last 120 years or so, we have, mainly within legal discourse, come to regard the fingerprint as an unmistakable - and 'unfakeable' - characteristic of the individual. In order to develop a typology of the criminal subject, men of science like Alphonse Bertillon and Francis Galton developed several procedures to examine the face and the fingerprint. The latter in particular gained a prominent position in the detection of crimes and the question of identity. As Georges DidiHuberman notes with the fingerprint in mind, it is remarkable, how a singularity with all its improbability became the valid symbol for identity (Didi-Huberman, 1999, 194). And yet, any identification is deeply dependent on the copy, in other words, authenticity is dependent on reproduction because identity can be confirmed by comparing it with several copies, for example from an archive.

The fingerprint is also closely related to the signature, and not just because in both cases ink leaves a trace or stain. As Francis Galton, Charles Darwin's cousin and one of the fathers of the scientific version of racism called eugenics, pointed out in his book on the fingerprint published in 1892, this personal characteristic was an esteemed way of signing letters in China and Bengal. Carlo Ginzburg, in his wide-ranging essay on traces, makes a connection with prophecy and fortune telling. He assumes that a culture that deciphers the drawings on a tortoise shell or the traces of birds must have also been able to decipher a fingerprint as a kind of writing (Ginzburg, 89). It was, however, the British colonial administration that took advantage of this practice in order to identify those subordinates of the Commonwealth who all seemed to look the same to them. As we all know today, this was the beginning of the fingerprint file - and, in connection with the archived photographic images of delinquents and suspects, of modern criminology (Ginzburg, 90).

The fingerprint, the face, and the signature are all still considered as the major characteristics used for identification (if we ignore extraordinary characteristics like scars). All three acquire their authentication, that is to say, their authorization, from a kind of 'mother copy'. Every signature and every fingerprint has to be more or less an identical copy of another one. But is there any original? Our faces change over the years, our signature always varies a bit. Authenticity, therefore, seems to be established in a process of constant repetition and constant comparison. During that process, identity is proven by similarity, which is proven by repeated visual examination. The substance used in order to obtain all these copies (including the printing of photographs of faces) - ink - is regarded more or less as neutral. In other words, the main quality of the substance that allows traces like footprints, signatures, and personal photos on passports, to be rendered visible is its 'invisiblility'. 


\section{Art and Brord}

In the following sections, I would like to slightly skew the picture a little bit by bringing in a different substance that is not at all neutral: blood. Compared to ink, blood seems much more auratic, even frightening, because blood is directly related to the body, either to the body of someone who voluntarily gives blood or has a blood test, or someone who has been wounded.

Blood has almost vanished from our everyday life. This has not always been the case, as the Italian social anthropologist Piero Camporesi has shown in his study on the 'Juice of Life'. In the Middle Ages and in early modern times, blood was much more a part of the public sphere. Today, in a society almost obsessed with privacy, and especially in times of HIV, blood seems too close to death and is therefore hidden behind the walls of hospitals and medical practices. This is confirmed by the pervasiveness of blood in action movies and crime or splatter movies. Blood evokes what in German is so aptly called 'Berübrungsangst' - a fear of touching, a fear of contact.

In contemporary art, the use of blood, of real blood - be it human blood or 'merely' animal blood - has almost always been regarded as a taboo-breaking act. It will thus come as no surprise that blood in art has often provoked a scandal. And I should add that I am referring to the Western Judeo-Christian art tradition. However, I cannot account for traditions such as Afro-Cuban syncretist rituals, for example. As 'material' - and one might even hesitate to call it a material in the usual sense - blood has been used mainly in performances, sparingly in Ana Mendieta's bodywork, while extremely lavishly, even wastefully, in Herman Nitsch's 'OrgyMystery-Theatre'. Not accidentally, most of these performances are interpreted as, or associated with, cult-like practices, and the inherent symbolism ranges from wounds and healing rituals to sacrifice and collective profligacy.

But there is more to the combination of blood and art than mere mimicry of religious undercurrents. What is at stake here is a revitalization of the aura, a re-auratization of the arts in the age of mechanical reproduction, namely in a way that relates art to Benjamin's notion of the 'Kultwert' (cult value) of the work of art. The definition of the aura as 'einmalige Erscheinung einer Ferne, so nab sie sein mag' (Benjamin, 1936, 142) [as the unique phenomenon of a distance, however close it may be (Benjamin, 1969, 222)] reconnects the cult value of the work of art to categories of apperception of time and space, and thus to the experience of an observer.

When Benjamin speaks of the 'Kultwert' of the art work, he seems to do so with the implicit intention of establishing it as a third term, in opposition or at least in calculated distinction to Marx's notions of Gebrauchswert (value in use) and Tauschwert (exchangeable value). This is made clear when Benjamin opens up his Work of Art essay, with reference to Marx's analysis of capitalist production. Production, as an economic practice, thus becomes the starting point for any reproduction or copy. Whereas, of course, the cult value is rooted in the ritual: 'Der 
einzigartige Wert des “echten” Kunstwerks hat seine Fundierung im Ritual, in dem es seinen originären und ersten Gebrauchswert hatte' (Benjamin, 143-144).

Given the fact that rituals are, by definition, acts of repetition, it is the object used in the ritual that gains its aura both through its singularity and its presence. In other words, the work of art derives much of its aura from its singularity or, at least, its rarity, and presence - which might sound somewhat banal. Let me first give an example. During the 1950s, the artist Yves Klein performed his so-called 'Anthropometries', a series of body prints on paper, carried out by naked female models 'dressed' only in colors. ${ }^{2}$

It is reported that Klein also used ox blood in this manner during one private performance. When he signed the paper rolls of bloody imprints of the models' bodies, he purportedly pricked his index finger with a needle and stamped the work of art with a fingerprint dipped in his own blood.

None of those pieces survive, however. According to his friend, the art critic Pierre Restany, Yves Klein destroyed them all shortly after the performance. $\mathrm{He}$ commented on this self-destructive act of iconoclasm by saying that he had the feeling that he had come too close to the devil (Wagner, 228-229). I mention this because of the somewhat strange echo of Benjamin's definition of the aura as 'a distance so near'. All the more so because Yves Klein once admitted, not surprisingly, that his 'Anthropometries' were inspired by a film on Hiroshima (showing, among others, the shadows of victims left on a wall) and the (lost) image of the Vera Icon, also known as the legendary sudarium of Veronica. This refers to the myth of the supposed actual image of Christ's face, called the Mandylion or the sudarium of Veronica, appearing as a 'print' on a piece of cloth. The original was said to have been preserved as a relic in Constantinople until 1204, and was then lost during the plundering of the city.

One tale regarding the origin of the Vera Icon relates the portrait to a woman by the name of Veronica (of course, an anagrammatic name) who is said to have been among the mourning women under the cross who, during Christ's journey to the Cross, gave him a piece of cloth to dry his face. According to this legend, the combination of sweat and blood from the suffering Christ created the first and only true portrait of Christ. And thus, the use of blood in combination with the imprint of body parts, in this case the face of Christ, can be traced back to this Vera Icon.

The relationship between the following example and the Vera Icon is predicated on the notion of blood-ink and the inherent symbolism of victim and sacrifice.

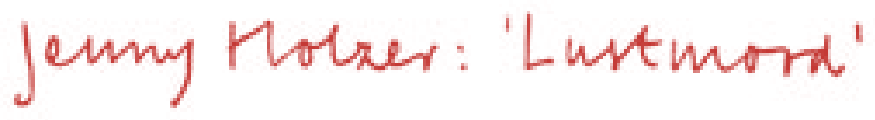

Jenny Holzer's 'Lustmord' (rape and murder) was a work commissioned by the German newspaper Süddeutsche Zeitung for their weekly supplement Süddeutsche Zeitung Magazin. Conceived in 1993-94 during the civil war in the former Yugoslavia, it is a reaction at that time to the constant news of the violation and 


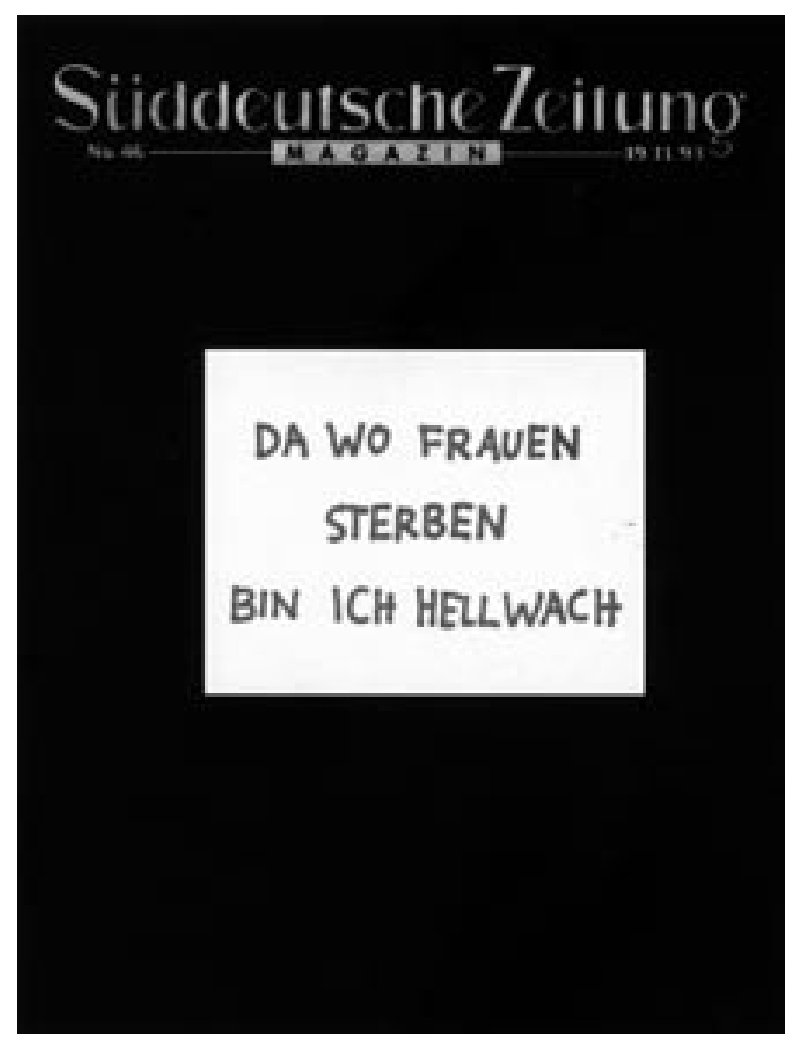

1. Frontpage Süddeutsche ZeitungMagazin, No 46, 1993.

rape of women. The result, however, is in no way a simple reaction, in terms of taking a position. 'Lustmord' presents three different viewpoints of a rape, namely the perpetrator's, the victim's, and the observer's. ${ }^{3}$ It is important to note that it is impossible to distinguish which position the artist herself has assumed.

Holzer uses cropped photographs of naked skin upon which she wrote various texts. We are not told whether these skin surfaces belong to a victim, or a perpetrator, or to some uninvolved bystander. I will not go into the details of the texts' content nor discuss the issue of violence and language, which is one of the topics of this piece. Instead, I would like to concentrate on the question of handwriting, print, and reproduction. Although written in neutral capital letters, it is clearly recognizable that Holzer's words are written by hand. The handwriting was carried out by means of a pen or a felt-tip marker. Some of the texts look like a tattoo, some appear blurred by sweat. The colors used are mostly red, blue, and black. It remains unclear, however, if the different colors represent, or are in any other way connected to, the aforementioned perspectives of victim, perpetrator, and observer. Nor is it possible to ascertain which part of the body has been used as a writing surface.

These handwriting samples have been reproduced twice: first, during the pho- 


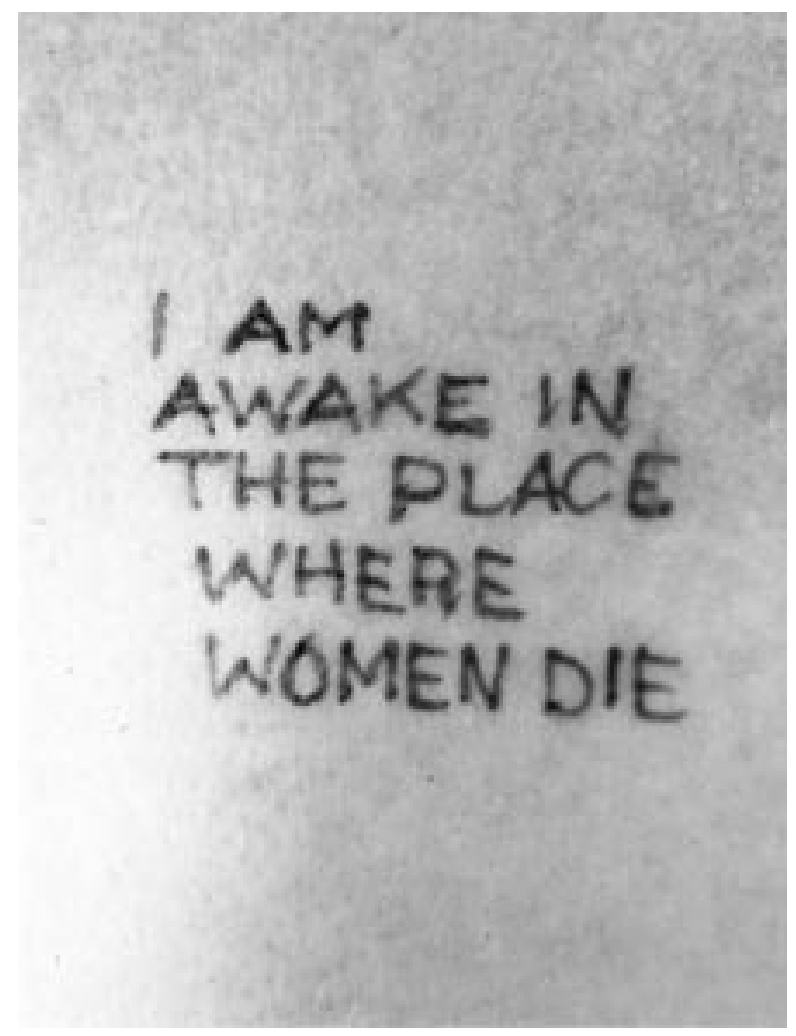

tographic session with the artist, and second, during preparations for the printing process of the insert. This is of some importance, as I will point out in a moment. The cover of the magazine with the inserted photographs again shows a text in scarlet red, but this time it is not written on skin. A white sheet of paper positioned over the black background of the magazine contains the following text: ' $D a$ wo Frauen sterben bin ich hellwach' ['I am wide-awake where women die']. This sheet of paper with the red handwriting was printed separately and glued to the cover. Then human blood donated by women volunteers from Yugoslavia was added by the artist to the red ink - not the pen ink, but the printing process ink. The beholder is not informed whether these donors were victims or not. Of course, this mixture of blood and ink was made public and of course it did cause a scandal, and, of course, the scandal was a calculated one.

Nevertheless, by reproducing handwriting, at least partly, and by using blood, the artist manages to rephrase the question of physical authenticity in a new way that reformulates the question of copy/reproduction and difference. While the original handwriting 'contains' only ink, the reproduction contains both ink and blood. Although we are unable to distinguish this difference, the implication, nevertheless made public knowledge here, is also meant to produce an auratic effect. 
The distant echo of the Vera Icon returns as a trace - the trace of handwriting, and of a hand writing. It is the trace of a 'blood toll' which has not been demanded in terms of heroic sacrifice, i.e., the martyrdom (of men), but is the result of permanent victimization (of women). ${ }^{4}$

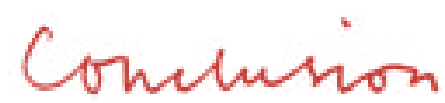

I conclude by recalling Bal and Bryson's presentation of the index-icon symmetry:

the iconic is a quality of the sign in relation to its object; it is best seen as a sign capable of evoking nonexistent objects because it proposes to imagine an object similar to the sign itself. Iconicity is in the first place a mode of reading, based on a hypothetical similarity between sign and object. (Bal and Bryson, 189)

The iconicity in Holzer's piece is therefore evoked via its association with blood stains, although there is no equivalent in terms of a real object that exists or has existed. There is no real blood in the game either. But, nevertheless, there is also an indexical procedure at work here insofar as the 'handmade' copies point to an absent 'original' that has left an impression.

In conclusion I would like to formulate a possible hypothesis: in all of my examples blood, this highly symbolic liquid, establishes an irritating oscillation between indexicality and iconicity. In terms of an artistic strategy, this serves as a kind of reinsertion of 'authenticity' into the realm of the copy. I conclude with the observation, formulated after Hal Foster, that the observer always witnesses some kind of uncanny return of the real.

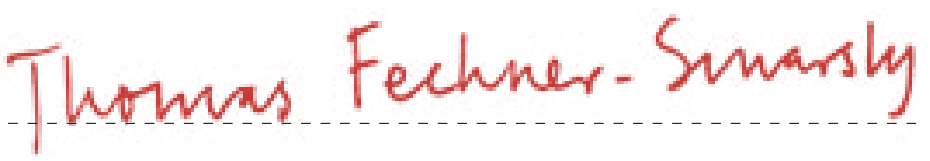

SIGN HERE! 
Notes

1. See Georges Didi-Huberman, Ähnlichkeit und Berührung, 190.

2. For Klein's Anthropometries, see Stich, 171-186.

3. This tripartite 'structure' seems crucial to me to properly understand violence and its images in a media society. As far as I know, there exists no systematic analysis of this constellation. See Susan Sontag's essay Regarding the Pain of Others. For the case of the Holocaust, see, for example, Raul Hilberg, Perpetrators, Victims, Bystanders. The Jewish Catastrophe 19331945.

4. The German term Opfer leaves it open to context and interpretation if a deed, a person, etc. must be regarded as sacrifice or victim.

\section{Works Cited}

Bal, Mieke and Norman Bryson. 'Semiotics and Art History', in: The Art Bulletin, June 1991, vol. LXXiıI, no. 2, New York: College Art Association, 174-208.

Benjamin, Walter. 'The Work of Art in the Age of Mechanical Reproduction', in Hannah Arendt (ed.), Illuminations. 217-251. Transl. Harry Zohn, New York: Schocken, 1969 (1936).

Camporesi, Piero. Juice of Life: The Symbolic and Magic Significance of Blood. London: Continuum, 1995.

Derrida, Jacques. Of Grammatology. Transl. and intro. by Gayatri Chakravorty Spivak. Baltimore: Johns Hopkins University Press, 1976.

Didi-Huberman, Georges. Ähnlichkeit und Berührung. Archäologie, Anachronismus und Modernität des Abdrucks. Cologne: DuMont, 1999. (German translation of DidiHuberman, Georges. L'Empreinte. Paris: Editions du Centre Pompidou, 1997).

Ginzburg, Carlo. 'Clues: Morelli, Freud, and Sherlock Holmes', in: Umberto Eco and Thomas A. Sebeok (eds.), The Sign of Three: Dupin, Holmes, Peirce. 81-118.

Bloomington: Indiana University Press, 1983.

Holzer, Jenny. Lustmord. Ostfilder: Cantz, 1996.

Stich, Sidra. Yves Klein. Stuttgart: Cantz, 1994.

Wagner, Monika. Das Material der Kunst. Eine andere Geschichte der Moderne, Munich: Beck, 2001. 


\section{Writing Over the body, Writing With the body: On Shirin Allah Series}

A close-up in black and white. A larger than life-size portrait of a woman covered in black, with a black veil. The veil only leaves her eyes and nose visible whereas her lips and neck are hidden beneath the black folds that frame her face and expands across the image's surface. The woman, with her heavily 'Oriental' made-up eyes, looks at the viewer directly; yet it is a fleeting glance. It is as if the camera's shutter was clicked just as the woman was passing by, capturing the very instant she glimpsed at the camera; this moment is further emphasized by her aslant posture. In her frozen stillness she does not challenge the viewer's gaze; her look does not force the viewer to avert her eyes. It is perhaps because she is facing the viewer through the Arabic letters written across the visible parts of her face, leaving only her eyes exempt from the scribbled lettering. Written in black-and-red ink, the text flows in circles in a loop left bare by the veil and ends (or begins?) just amidst her eyebrows, becoming as hypnotic as the eyes looking at the viewer (Illustration 1).

Another monochromatic portrait of a veiled woman: Here the veil renders her face visible but covers her from head to shoulders. Like the first woman, she stares at the viewer directly, yet hers is a confrontational gaze. It is a straight look that will not be interrupted; she is not going to blink. She holds a gun right in front of her face in her invisible hands (outside the image frame, presumably under her veil). The gun splits her face in two, as well as the Arabic text inscribed on it. Unlike the first one, the text in this image is written in black ink in a linear horizontal manner, again leaving her eyes untouched (Illustration 2). 


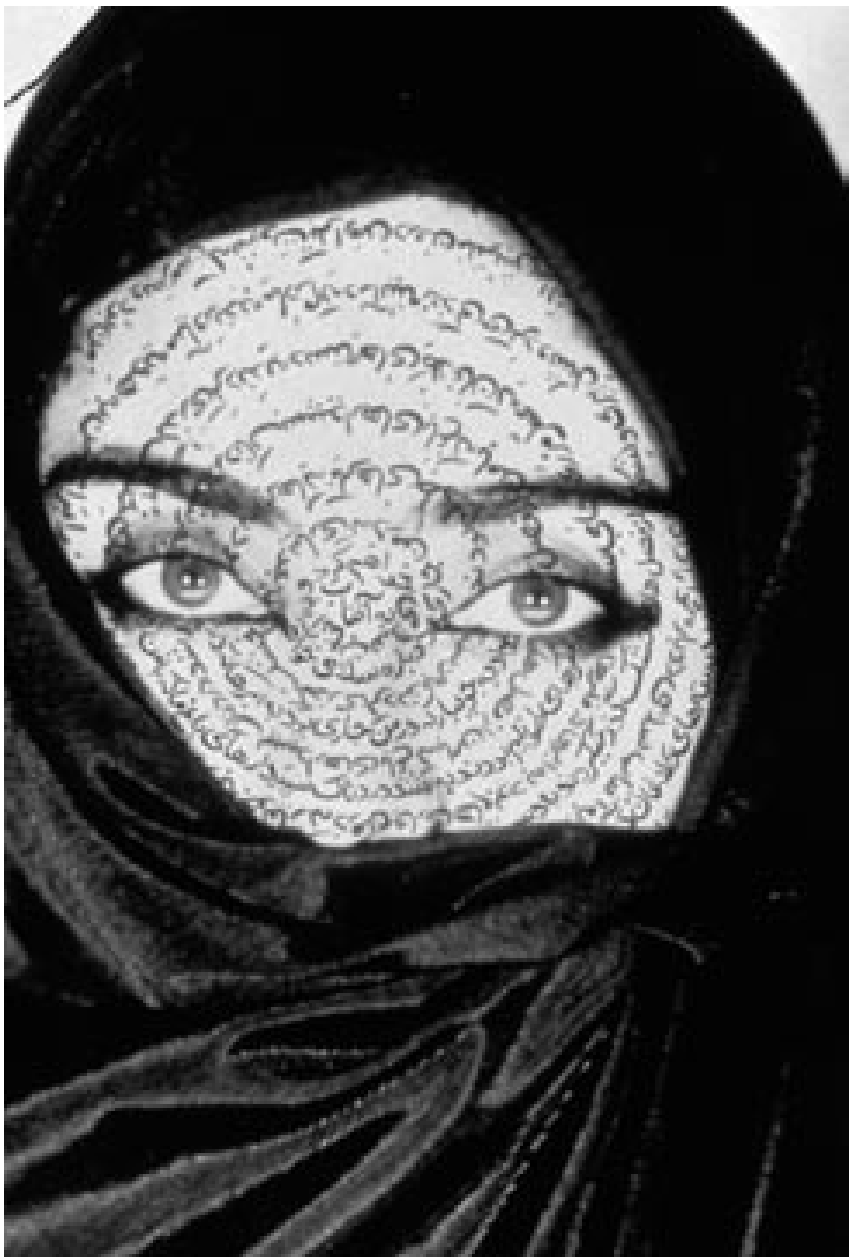

1. Shirin Neshat, I Am Its Secret, 1993

These two images, I Am Its Secret and the latter Rebellious Silence, are part of Shirin Neshat's photographic series Women of Allah. Neshat is an Iranian-born artist living and working in New York. According to Hamid Naficy, Neshat is among a group of 'intercultural artists and filmmakers in the West whose lives and works intersect artistic domains (in her case, photography and film) and cross boundaries of style, genre, gender, race, nationality, ethnicity, language and culture' (2000, 43). Neshat left Iran well before the Islamic revolution in 1979, and returned for the first time in 1990. Three years after that visit, she started the monochromatic photo series Women of Allah and kept the project going until 1997. In Neshat's words, the series focuses on 'the Islamic revolution in Iran and particularly on the subject of women in relation to violence and politics' (Desai 2003). The photographic series is composed of images of women, mostly of Neshat herself, wearing veils and carrying guns with Farsi calligraphy written all over them. Most of the 


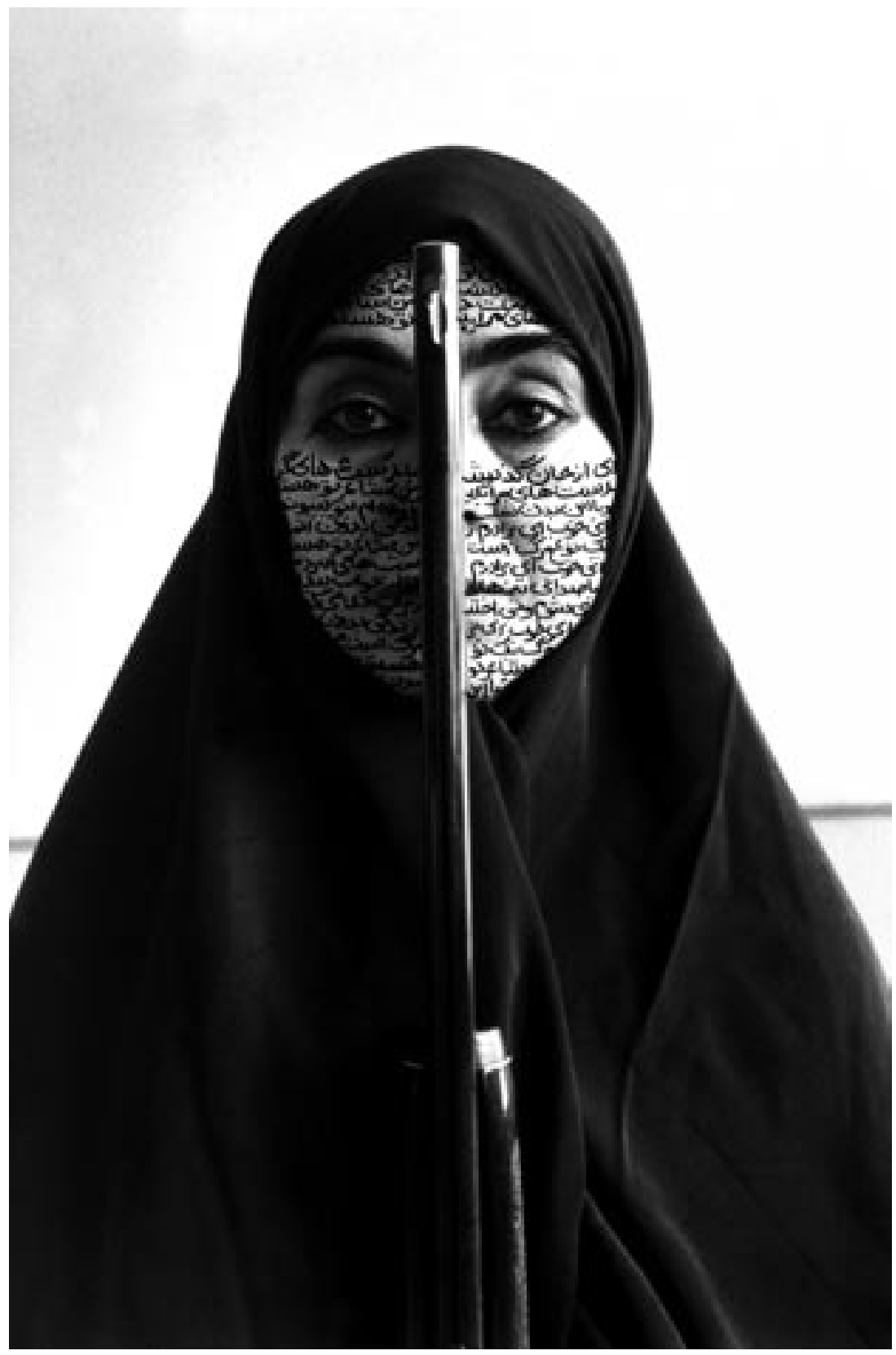


time, the texts are written across the visible body parts, especially on the face, the hands, and the feet - the only parts of a woman that are allowed to be visible according to the mandatory dress code. Yet, contrary to the potential first impression that might place the handwriting immediately on the body, the text in the series is written over the images, not on the bodies of the photographed women. As such, the handwritten inscriptions open up a medial space between the body and the writing that provides the images with multi-layeredness. Within the Women of Allah series, it is among these layers that handwriting as a cultural practice reclaims its aesthetic, cultural, and political reminiscence and constructs the core of this article.

Given that Neshat is an artist working and producing in the heart of the contemporary art scene, it is plausible to argue that her works are on display primarily for the Western viewer. ${ }^{I}$ Veils, guns, and Farsi inscriptions are highly loaded signs that invoke in the viewer a historically constructed, multi-layered image of Muslim cultures. Viewed from the present - especially in the aftermath of 9/11 in the us and its global reflection - the veil does not refer to the 'exotic' unknown body of the Oriental women anymore; instead it refers to the hotly disputed issues of Muslim women's emancipation and to the dangerous female 'terrorist' carrying a hidden bomb on her body. Guns are not only a reminder of local conflicts in the Middle East but are also an echo of Islamic upheaval, global terrorism, and jihad and thus a 'war on terror'. ${ }^{2}$ Such echoing is further re-enforced by the Arabic inscriptions which may carry different significations such as references to the Quran, sharia, or to the 'repressive' Islamic states, or to the captions accompanying images on the famous television network Al-Jazeera and daily news images from Iraq on the global or national media.

The Women of Allah series does not only refer to Iran and Iranian women but are also generic images for the 'Muslim Other', materialized in the female body as the site of visual symbols made to represent geographically and historically diverse Muslim societies. On the surface, the images do nothing but reproduce the historically constructed fantasy and fear of the Orient by employing the overused signs of the Orient. However, I interpret these images as a series that problematizes this generic and immediate reading and challenges the Orientalist gaze by appropriating these very Islamic symbols in a deconstructive manner. By masking/ornamenting her culturally hybrid body with the veil and the Arabic script of the Muslim Other, Neshat questions the Western viewer's already constructed viewing position and attempts to turn the gaze upon itself to reflect on the 'cultural screen' upon which these images are encouraged to be seen. ${ }^{3}$ Yet, the images remain stereotypically Oriental for the viewer regardless of the artist's intentions. The viewer can only see what she already knows in accordance with the frames made available for interpretation. In their stillness, these images do not invoke such critical self-reflective readings. Yet, images do not stand still; they metaphorically move and along their movement they encourage the viewer to move with them. Thus, I argue that the Farsi calligraphy written across each image in a rhythmic fashion has the capacity to tickle the eye of the viewer and in that capacity encourages an engaged reading 
of the image of the Muslim Other, albeit in a problematic manner that I will discuss below.

The handwritten text on the Women of Allab images comments on the possibility of cross-cultural viewing positions at the intersections of the visual and the verbal, of looking and reading, of translation and unreadability, all of which convene on the body of the artist that is inscribed with calligraphy. At the crossroads, the super-imposed writing opens up multi-layered interpretations by creating productive tensions first by appropriating and remediating the Islamic calligraphy tradition on the visual plane, and second, by reclaiming the body both as a text that is culturally overwritten and as a medium that self-reflectively overwrites itself.

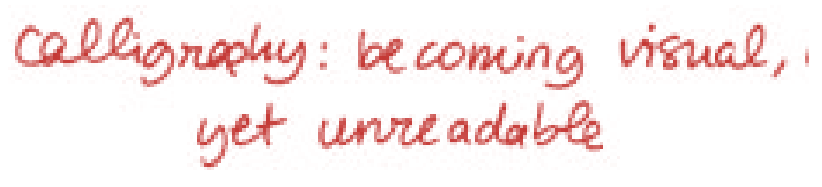

What is, perhaps, most immediately striking about the series are the texts in Farsi calligraphy super-imposed over the images. Delicately written in circular forms opening out or perhaps closing in, almost hypnotizing in its effect, like a swirling black-and-white wheel, the text on I am Its Secret (Illustration 1) pulls the viewer into the depth opened up on the surface of the image. Similarly, the floating letters on the face of the woman in Rebellious Silence (Illustration 2) tease the viewer to tackle the text, just behind the rifle, on the inside of the veil, in front of the white background.

What catches the eye is the additional visual dimension, a supplementary space attached to the flat surface of the photographic image. This new space expands from the tactile materiality of the ink, by means of its thickness striking out of the image, beyond its textual character breaking up the interaction of the visual signs that disturb the continuity of a comfortable viewing. In this mixed space, the viewer is tempted to look, to read, and to touch the calligraphic texts. By appropriating the Islamic practice of calligraphy and by attaching an 'othered' cultural medium of writing onto the surface of the photographic image, Neshat also reappropriates the synaesthetic experience of the calligraphic tradition. What I find intriguing in such an invitation of viewing is the juxtaposition of two modes of seeing, that of looking and reading, both on the level of the image as a whole and over the written space alone.

The confusion between modalities of looking and reading expands outwards from the calligraphic text, in front of which the non-Farsi reader/viewer finds herself unable to read, towards the image, and thus merges visual signs into verbal ones and vice versa. This intermingling of modalities is one of the recurring traits of an encounter with the Islamic calligraphy tradition. Calligraphy has been the most important practice of the visual arts within Muslim cultures, and is one that populates the visual field in forms ranging from manuscript writing to architectural facades and building interiors carved onto stone, almost like an ornamental 
graffiti, or framed and hung like a painting. Regardless of the surface it ornaments, the main objective of calligraphy within Muslim cultures is to convey a message of a strictly linguistic order. As a practice of manuscript reproduction, calligraphic writing definitely communicates the written in a legible form. Even practiced as 'pure' architectural decoration, calligraphic texts may still be 'informative inscriptions' (Grabar 1978, 116-117) that completely fulfill their linguistic function of transmitting a message, often a religious one. Nevertheless, as Valerie Gonzales states, calligraphic inscriptions can also go beyond 'this universal function of writing and completely lose their function of objective linguistic signaling by transforming themselves into illegible forms, sometimes even into meaningless epigraphic type called "pseudo-Arabic"” (Gonzales 2001, 99) where beauty, complexity and sophistication gain prominence. In this sense, most of the decorative Islamic calligraphic works, whether on an architectural space or on the surface of a simple object, tend to subordinate the linguistic and semantic functioning to a visual function that invokes the aesthetics of writing and also that of the pleasure of looking.

In such works, the exaggerated rhythm, the excessive decoration and the overstated figural manifestation of the lettering overrides the intentional content of the text. This transformation from verbal to visual, then, interrupts the interplay between the signifier and the signified on the scriptural level and encourages an iconographic reading brought on by the highlighted visuality of the inscription that makes the text illegible, yet visually stimulating. When calligraphy is so elaborated or distorted and manipulated that it can hardly be read, it suggests to the pious Muslim a well-known Quranic text or literary work, or a religious phrase, and it lets the viewer's memory and imagination supply the rest. In this respect, the experience of the viewer of the calligraphic works is beyond a simple act of reading; it is an aesthetic experience that is collectively shared on the basis of a performance of cultural memory and as an enactment of individual revelation. 4

In her Women of Allah series, Neshat complicates the visual tradition of calligraphy in a very similar manner. Neshat here imposes the aesthetics of calligraphic writing onto the photographic image and the very corporeality of the letters opens up an aesthetic scriptural space on the visual surface. As such, the images suggest a viewing position that oscillates between reading and looking at the image. As stated before, this intermingling of modalities of seeing is one of the main traits of Islamic calligraphic practice. Yet, in Neshat's series, the juxtaposition of the modalities gains cross-cultural significance. If the photograph represents the triumph of the Western eye by confirming the perspectival laws which so long constituted the Western norm of vision, as argued by Comolli and others (quoted in Silverman 1996, 127), Neshat reclaims this primacy in Islamic cultures of the visual over the written word in which the divine 'invisible' truth reveals itself by means of the spatio-temporal materiality of speech and scripture. In the series, then, two different signifying systems carrying their respective cultural connotations conflate, or, are translated into each other's language, by highlighting the visual character of the written and infecting the visual with the stain of the verbal. 
However, it is less the act of reading than that of looking that overrules the image as the non-Farsi viewer/reader of these images is left helpless in front of them, primarily because, in her exhibitions, Neshat usually does not provide translations of the texts. ${ }^{5}$ Although the script is written in clear and legible Farsi script, it remains completely unreadable to the non-Farsi reader. The scripted letters ask to be read, as it were; the viewer is invited to be the reader of the text while the reader's inability to read them is already presumed. The act of reading is both promised and prevented, which creates a desire to read that will not be fulfilled. From such a paradoxical viewing position, the calligraphic inscriptions function in a manner similar to that of the highly manipulated and distorted traditional decorative Islamic calligraphy that becomes illegible. Through Neshat's remediation of the calligraphic tradition, where unreadability is emphasized in the absence of a readable text, the inscriptions on the photographic images encourage a diverse mode of signification rather than referring strictly to the linguistic signifiers. For the viewer of these images, confronted by their unreadability, the calligraphic text becomes a generic visual sign, much like the veil and the gun now do in a homogenous manner. Resembling the experience of the Muslim in front of the illegible calligraphic texts in which the illegible signifies a well-known religious text or familiar phrase, Neshat's handwriting encourages the viewer to interpret the images in a similar way. The immediate 'reading' of the unreadable Farsi text relates to the Quran in a way similar to a Muslim standing in front of an illegible text. In an interview, Neshat stated that she has often been asked if the inscriptions were taken from the Quran and, according to curator Igor Zabel, this seems to be a natural question, 'given the prevalence of stereotypes about Islamic fundamentalism, in which the Quran is compulsory - the only possible - text' (Zabel 2001, 22). For the Western viewer any reference to Quran, as the 'only text' governing the Muslim world, would invoke the notion of oppression, backwardness, and eventually religious terrorism in relation to contemporary Muslim societies. Especially since the texts are written over the female body, the viewer may assume that the texts were quotations pertaining to the status of women and the association would invoke that of the suppression of and the violence against women, as well as evoking issues of freedom and equality within Muslim societies. ${ }^{6}$

However, Neshat plays with the potential signification that is at work here. The texts that Neshat quotes - visually and literally - are taken from famous Iranian feminist poets' works and most of the time they are critical of the masculine ideology of contemporary Iranian society.7 Certainly, most viewers are excluded from the meaning of the text because they are unable to read the Arabic script and understand the Farsi language. Yet, I believe that this potential viewer disability is exactly what Neshat is hinting at, supported by the unavailability of the translated texts in the exhibitions. The viewer who fails to read the image in the absence of a translation interprets it through an Orientalist discourse that defines the Muslim Other by means of historically constructed culturally mediated stereotypes. In fact, this encouraged 'misreading' implicitly whispers to the viewer that rather than the veil concealing the body, it is the Western discourse about the Muslim 
world that obscures the viewers' eyes. From the unreadable text of the so-called Orient written on the female body, the silence turns into a rebellious scream that expands towards the veil, to the gun, to the non-white female body and questions the dynamics of the cross-cultural viewing positions and the possibility of visual translation.

In fact, the female body, mostly that of the artist herself, demands interpretation as much as the calligraphic texts do. The body in these images is an effect of translation and transformation from the 'natural' feminine body to a bearer of culture; from the site of submission to a surface overwritten by resistance; from the generic anonymous Oriental body to the culturally marked female body. And all these translations take place at the convergence of the handwritten texts that contour and fill the images with the bits and pieces of a fragmented female body.

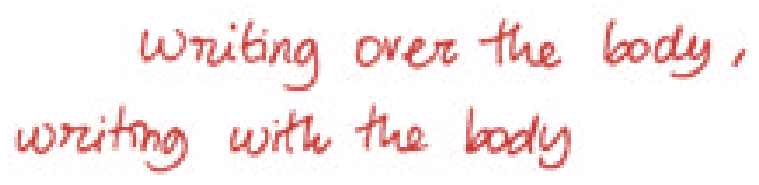

The body, as Foucault argues in Discipline and Punishment, has been historically and discursively constructed as a site of contesting power structures. Throughout his analyses, Foucault shows how the body, in its very materiality, does not stand in external relation to power, but is 'marked, stamped, invested, acted upon, inscribed, and cultivated by a historically contingent nexus of power/discourse' (Yeǧenoğlu 1998, 113). Accordingly, in the history of Islamic societies, the body, particularly the female body, has constantly emerged as a controversial symbolic site of these contestations of power structures, primarily by attempting to regulate its activities by prescribing clothing practices. In Iranian society, while the unveiled female body was turned into the carrier of the 'modern' Pahlavi regime, the re-veiling of this body in the context of the Iranian Islamic revolution served as a compulsory statement of its ideology. However, it is almost impossible to understand the 'internal' politics of the veil in isolation from its colonialist and neo-colonialist construction and politics, as in the cases of Algeria and Afghanistan, for example, where stripping women bare from the veil is seen as a form of emancipation. In both cases, the veil becomes a visual sign of difference that demarcates the 'Muslim' women from the 'Western' other in a similar manner that it severs inside from outside, private from public. From the Western perspective, the difference of the veiled body is constructed by means of the formulation of a lack, the lack of agency, as well as the passivity of a silenced and oppressed invisible body contrasted with the presumable truth and naturalness of the unveiled Western body. ${ }^{8}$

However, in the Women of Allah series, the veiled feminine body as the object and target of power gains a different signifying power through the calligraphic inscriptions, which translate the body into a culturally engraved site of resistance. The quoted inscriptions, in a subtle way, suggest that the feminine body has multiple cultural skins with multiple significations: the material veil, which both 'erases 
and enforces embodiment' (Moore 2002, 3) and the textual skin that overwrites the body that both suppresses and enables the subject with creative agency and cultural identity. It is between these skins - including the skin of the image - that the body is transformed and translated at the very moment when the non-Farsi reader/ viewer fails to give a voice, and hence meaning, to the text that repels the undemanding cross-cultural viewing position. As I suggested before, the handwritten inscriptions that the viewer fails to interpret or 'read' as verses from the Quran, are, in fact, excerpts from the works of contemporary Iranian writers, such as Forugh Farrokhzad, Tahereh Saffarzadeh, and Monirou Ravanipur, whose works have feminist overtones and are mostly in stark opposition to the ideology of the Islamic Revolution. The handwritten quotes from these 'militant' writers, then, imply that the culturally inscribed feminine body is not only a site for subordination and coercion but is also a space for creative and subversive processes. In this capacity, the images suggest that the viewer read the female body as a cultural text that is entirely overwritten, yet it is not the body of the victim anymore; it is a culturally inscribed body that is also a site of memory that remembers the repressed stories and does not shy away from showing it. However, the visible part of the text that is exposed by the frame of the veil does not communicate with the viewer, who, in turn, questions the universality of the body as text that can be read crossculturally.

Aside from problematizing the cross-cultural reading of the generic anonymous body of Muslim women, Neshat also challenges her culturally hybrid multiple skins by writing over the image of her own body. It is less by means of (dis)guising her body as the veiled cultural other than by transcribing texts in her mother tongue over and over onto the image of her body that Neshat negotiates and appropriates her diasporic, or exiled, Iranian cultural identity. Writing is what carries culture in much the same way that handwriting identifies individuals. Nehsat's work deals with both the personal and the cultural. By writing in Farsi she reclaims herself in terms of a culture that pays homage to the written word. Like the humble calligrapher who writes the 99 beautiful names of Allah, or basmalab over and over again, Neshat fills in her blank face, the face without cultural remarks, with the roundness and loops of the Arabic letters. In this respect, I read the artist's deployment of the practice of writing over the body, and especially on the face, as an implicit appropriation of the repressed tradition of Hurufism (harf, pl. huruf: letter) that sublimates the divine character of the Arabic alphabet and the practice of its inscription. ${ }^{9}$ The Hurufi sect reworks the Islamic belief of the Divine Pen (al-Qalam) by writing the realities of all things (al-haqa'iq) upon the Guarded Tablet (al-Lawh al Mahfuz) and upon the pages of that archetypal book that is none other than the Quran, and further believes that the human body, and especially the face, is a text that is already written by the divine. According to Hurufi belief, the main elements of the face contain the mystical and the divine letters of the Arabic alphabet. By 'reading' the divine text on the face, the Hurufi unravels the divine truth, Allah. As important as unraveling the divine truth, the Hurufi practice of reading and interpreting the face is a practice of knowing the self. In line with this understanding, the Hurufi calligraphic tradition asserts itself as the ultimate figuration of writing, 
or vice-versa, where the human face is twice written: first with the divine letters, and then using calligraphy, in the search for the self and the divine creator. ${ }^{I O}$

Reinterpreting the Hurufi tradition, I read Neshat's works as a search for the self through the cultural practice of writing with the body over the body. By writing the letters of the alphabet of her mother tongue, the artist negotiates Iranian cultural identity and traces her hybrid body with the cultural text of Iranian heritage. This is to say that, instead of searching for unification with the divine by means of individual revelation, Neshat's practice of writing is directed more towards appreciating her diasporic difference through the performance of writing as an established cultural practice. As a result, handwriting becomes both the artist's individual signature and a sign of her cultural hybridity.

However, Neshat does not apply the text directly over her body; she does something quite different. Neshat first photographs herself and then writes over the images of bits and pieces of her body. By doing so, she manages to keep the handwriting authentic and directs attention towards the materiality of writing, and hence towards the body at work, the 'hand' of writing. As such, the lost body of the artist as the photographed or the photographer is brought back by the handwriting that carries the trace of the writing subject on the mechanically reproduced images. The subjectless gaze of the camera that turns the female body into an object of looking is countered by the writing subject and by its corporeal energy, stimulated by the rhythmic inscription of Arabic letters. The formality of calligraphic writing, that is, the flow of letters from right-to-left, the consistency of linear movements, which are always horizontal, directional, and dynamic, becomes an indexical sign of the artist's body executing the text. The manual gestures of the author that oscillate between visibility and invisibility while connecting one letter to the other or separating them from each other, expose the kinetic impulses underlying the act of inscription. From the hand that holds the pen, the movement of writing expands outwards toward the wrist, the elbow, and to the shoulder of the inscriber, and embodies the artist while her hand mimics the shape of Arabic letters. The rhythmic pulses of the letters, their morphing over time, the pressure of the pen, and the thickness of the ink that it leaves on the surface of the photographic image, refer to the artist's body in labor in durée of its practical activity. The writing body of the artist that fills the image with temporality also reclaims the generic disembodied image of the Muslim body with an embodied one that is culturally submitted to a regime of gestural/ scriptural training.

As Foucault writes in Discipline and Punish, good handwriting 'presupposes a gymnastics - a whole routine whose rigorous code invests the body in its entirety, from the points of the feet to the tip of the index finger' $(1979,152)$. In the Women of Allah series, Neshat's hand, endowed with proper orthography, almost struggles with the practice of writing that has disciplined her body. The viewer is left to observe countless lines flowing in every direction in each image, in which the meticulous process of writing almost appears as a bodily torment where the artist negotiates the Iranian cultural heritage at pains. Within such negotiation, the calligraphic texts mediate between the artist, as a unique individual, and her relationship with the cultural community to which she belongs. In this sense, the script as a cultural 
indicator that ornaments the visible parts of the fragmented bodies in the images generates a productive space in which the artist deconstructs and reconstructs her cultural identity so as to appropriate her hybrid body text.

Moreover, the artist's writing body is further emphasized by the direct inscription of the texts over the images. Authentic handwriting, in contrast with the endless reproducibility of the photographic image, confirms the writer's individual uniqueness, distinguishing her as a signature and signing her presence at the unique moment of writing. The handwritten text represents the willingness of the artist to communicate with the viewer/reader on a very personal level; the act of writing is almost a confession of the artist as a way of revealing her identity by means of the inclusion of the autographic marks on the images of herself. By doing so, the artist unifies the mechanical image of herself with her bodily self through the act of writing, exchanging blood for ink, fingerprint for pen.

The authentic handwriting not only signifies the uniqueness of the writer but also provides the images with a uniqueness of their own, which otherwise they would not have had. Of the many possible copies, the overwritten ones become the original that distinguish the images from any other photographic work. The photographic negative as the original source of infinite copies hence loses its primacy and is replaced by the authenticity of the written images. In this respect, each and every image in the series becomes unique and repudiates its reproducibility and reclaims the authority that is lost with the prospect of reproduction. As is well known, in his essay The Work of Art in the Age of Mechanical Reproduction, Walter Benjamin asserts that, by means of the process of mechanical reproduction, the work of art loses its aura, that is, its historical uniqueness, as well as the unique viewing experience of such works. Benjamin does not mention handwriting or calligraphy as an aesthetic experience that would re-invoke the concept of an aura that is lost because of its reproducibility. Although he hints at lithography and print technologies with regard to writing, it is obvious that he does not consider handwriting as having aesthetic qualities and hence capable of inducing aura. As Sonja Neef and José van Dijck state in the introduction of this collection, this lapse in Benjamin's article is revealing, since it neglects the unique visual aspect of handwriting on the one hand and fails to notice the effectiveness of the mechanical reproducibility of handwriting on the other. However, once I have invoked the imageness of calligraphy at work, I find the discussion of the concept of aura productive with regard to Neshat's calligraphic inscriptions.

As I mentioned earlier, the materiality and the plasticity of the unreadable calligraphic text attract the viewer's eye almost in a hypnotic manner towards the scriptural plane it opens up. However, in contradiction to other familiar cultural signs, such as the veil, handwriting presents itself as intimate while keeping its distance. This intimacy, or closeness, is brought on by the images provided by the media from different parts of the Middle East while the letters enjoy the distance of an 'unheard-of symbolic system' as Roland Barthes $(1982,2)$ would say. Yet the distance does not stem from viewers' failing to read the text but from the sudden embodiment of the artist potentially inscribing verses from the Quran like a talisman, evoking the ritualistic and mystical dimensions of writing, which subordinates the 
'emancipated' photographic image to the cult-like value of the written. In this capacity, the handwriting re-enchants the demystified photographic image that provides the image with inapproachability, in line with Benjamin's definition of aura as a 'unique phenomenon of a distance however close it may be' (222). Moreover, handwriting pulls the photographic image towards the domain of tradition both by emphasizing handwriting's historical function and by invoking the tradition of calligraphic practice. Taken together, the images in the series are covered with an auratic shield that puts them at a distance from the viewer, making the images difficult to consume all at once. The physical distance one has to maintain in front of the larger than life-size images is, then, accompanied by a psychological and cultural distance enforced by the handwritten texts. The imposed distance alters the experience of the contemporary viewer for whom the Muslim women, brought close by the relentlessly repeated media images, are seen through the Oriental/colonial camera that shoots them as powerless veiled victims. Instead of conjuring up the closeness of the already known that would only make the veil transparent in order to expose the submitted female body, the auratic distance encourages the viewer to see beneath the opaque veil in order to read the unheard feminine voice that resists. It is along these lines that I interpret the titles such as Speechless (1996) or Rebellious Silence; even if Muslim women speak and write in their own language, their words remain mute, not because they are unreadable but because they are unheard. In the way that the right-to-left lines of text written across the Rebellious Silence speaks out: 'stories of your martyrdom / like martyrdom of the people / remain unheard. / They have no voice, no image, no date, / they are unannounced'. ${ }^{I I}$

In the Women of Allah series, Shirin Neshat tries to give a voice, an image, and a date to the Oriental woman by writing over the cross-cultural tensions. Yet, this time, the viewer remains silent because she cannot give a voice to the unreadable handwriting.

\section{Beguim Orden Firat}

SIGN HERE! 
Notes

1. Shirin Neshat has exhibited widely in major European and North American cities. Among her most recent solo exhibitions are those held at the Miami Art Museum (2003), Houston Museum of Contemporary Art (2003), Museum of Modern Art, Mexico City (2003), Castello di Rivoli in Turin, Italy (2002), and the Walker Art Center, Minneapolis (2002). She has been included in numerous international exhibitions and film festivals, including Documenta 11 in Kassel, Germany (2002), Sundance Film Festival (2003), Tribeca Film Festival (2003), Locarno Film Festival (2002), 48th Venice Biennale (1999), 2nd Johannesburg Biennale (1997), and the 5th Istanbul Biennale (1995). Conversely, although her works deal with Muslim societies in general, and with Iranian society in particular, they have never been displayed in Iran or in most of the other Muslim societies. With such an audience profile, I find it plausible to argue that Neshat not only produces in the West but also for the Western viewer. Therefore, throughout the article, I use the term 'the viewer' to refer to the Western viewer, the main consumer of Neshat's art.

2. As Jaqueline Larson (1997) points out, in some of the photographs, like Stories of Martyrdom (1994), the brand Remington, an American firearms company, is clearly visible on the rifle. For Larson, the visibility of the label in the image refers to the US production of the images such as that of the US-produced rifle, and she interprets the image in relation to the persistence of the American gaze and to what America expects to see. Aside from this possible interpretation, I think that the visibility of the label also comments on the global arms trade and on the US's role as the exporter of arms and the source of conflict in certain parts of the world, in particular the Middle East.

3. I use the term 'cultural screen' in the way Kaja Silverman interprets the Lacanian visual field. The cultural screen, as understood by Silverman, is 'the culturally generated image or repertoire of images through which subjects are not only constituted, but differentiated in relation to class, race, sexuality, age and nationality' $(1992,135)$. In the Lacanian model of the field of vision, the screen stands between the subject (of the look) and the infamous gaze. Therefore, wherever the subject looks, she sees through the framework of the cultural screen including herself and the cultural other.

4. Since the written, as a transmission of the divine words of Allah, has been given primacy in Muslim culture, calligraphic writing, as well as its experience by the viewer/reader, is usually explained in terms of individual revelation. See, for example, Annemarie Schimmel, Calligraphy and Islamic Culture, London: Tauris, 1990 and Mystical Dimensions of Islam, Chapel Hill: University of North Carolina Press, 1975; where she discusses the mystical dimension of Arabic letters at length. See also Sayyed Hossein Nasr, Islamic Art and Spirituality, New York: State University of New York Press, 1987.

5. To my knowledge, the translations of the texts were not included in most of the exhibitions, although there have been exceptions like The Serpentine Gallery, London (July 28 - September 3, 2000) where the translations were provided in the exhibition hall, or the Women of Allah exhibition in Artspeak Gallery, Vancouver, Canada (April 25 - June 7, 1997) that included the translations, not in the exhibition itself, but in the catalogue. Neshat also does not provide the subtitled translations of later video works that followed the Women of Allah series.

6. As I was writing this article, Theo van Gogh, a Dutch film director, was assassinated, by a 'fundamentalist' Dutch-Moroccan Muslim. The media claimed that he was killed because of the film Submission (2004) that Van Gogh made in collaboration with Ayaan Hirsi Ali, a member of the Dutch Parliament and the conservativeliberal party VVD. The ten-minute film deals with the oppression of and violence against women in the Muslim world and narrates stories of women beaten and raped by their family members. It includes shots of women with their faces halfveiled, wearing a transparent black chador-like clothes that makes the overwritten Quranic verses - dealing with the situation of woman on their bodies, visible. Moreover, one hears invisible whip lashings while the camera wanders through the naked bodies overwritten and freshly wounded by lash strokes. Judged as highly controversial at the time of its broadcast on Dutch television, I believe the film is very superficial in how it discusses women's rights in Muslim societies. Resembling the Women of Allah series in the way 
it deals with signs of Islam, Submission offers a rather simplified stereotypical view that is far from complicating and discussing the issues at stake. As such, contrary to Neshat's work, the film remains mere provocation that does not lead to a productive dialogue. For a discussion of the phenomenon of Ayaan Hirsi Ali and the film Submission in Dutch socio-political context see, " "Word alsjeblieft wakker": Submission, het fenomeen "Ayaan" en de nieuwe ideologische confrontatie' by Marc de Leeuw and Sonja van Wichelen and 'De obsessie met Hirsi Ali en de broodnodige kritiek op het neo-liberalisme' by Ingrid Hoofd in Tijdschrift voor Genderstudies 2005 (4).

7. The text that overwrites Rebellious Silence is a poem by Tahereh Saffarzadeh (1936) entitled 'Allegiance with Wakefulness' (1980). The quote over the surface of I Am Its Secret is an excerpt from Forugh Farrokhzad's (1933-1967) poem titled 'I Will Greet the Sun Again'. Farrokhzad's poetry had an immediate impact in preRevolutionary Iran because of its seductive tone and subject matter. In contrast to the emotional and sexual characteristics of Farrokhzad's writings, Saffarzadeh's poetry celebrates the martyrs in the Iran-Iraq war and is emphatically prorevolutionary Iran. She was also one of the few women in the Iranian parliament to represent women's issues after the revolution.

8. For a theoretical discussion of the relation of the veil and embodiment see, Meyda Yeğenoğlu, Colonial Fantasies, 112-120. For an analysis of how the veil is articulated in nationalist projects in Algeria and Turkey, elaborated through Partha Chatterjee's framework provided in Nationalist Thought and The Colonial World, see, again, Yeğenoğlu, Colonial Fantasies, especially the chapter entitled, 'The Battle of the Veil: Woman Between Orientalism and Nationalism'.

9. Hurufism or Hurufiyya is an unorthodox Muslim sect of gnostic-cabalistic tendencies founded by the Iranian mystic Fadl Allah of Astarabad, who was flayed to death for his heretical beliefs in circa 1401. According to The Encyclopaedia of Islam, one of the tenets of the Hurufi doctrine is the idea that God reveals himself in the Word and that words that are made up of sounds are always associated with letters. The total number of letters (and their numerical value according to the abdjad) is the total of all emanating and creative possibilities of God and is God himself made manifest. For the relation between calligraphic tradition and the Hurufi influence, see Annemarie Schimmel, Calligraphy and Islamic Culture.

10. Annemarie Schimmel notes that the lasting influence of the Hurufis, 'who had systematized the equations between letters of Arabic alphabet and features of the human face or the human body' on the scriptural pictures of the Bektashi order, has strong Shi'i influences. Schimmel gives examples of scriptural pictures that show human faces comprised of a mirrored Ali and the names of Panjtan (Muhammad, Ali, Fatima, Hasan, and Husayn) and Allah. See, Annemarie Schimmel, 'Calligraphy and Sufism in Ottoman Turkey', 247-248.

11. The translation of Tahereh Saffarazdeh's poem 'Allegiance with Wakefulness' is taken from Artspeak Gallery's exhibition catalogue Shirin Neshat 'Women of Allah' on page 28 .

\section{Works Cited}

Benjamin, Walter. 'The Work of Art in the Age of Mechanical Reproduction', in: Hannah Arendt (ed), Illuminations. 217-251. Transl. Harry Zohn, New York: Schocken, 1969 (1936).

Barthes, Roland. The Empire of Signs. Trans. Richard Howard, New York: Hill and Wang, 1982.

Desai, Vishakha. 'A Conversation with Shirin Neshat and Shahzia Sikander', 2003, in: March 2004. Available at: <http://www.asiasource.org>.

The Encyclopaedia of Islam, New Edition, vol. III, H.A.R. Gibb, et. al (eds.), Leiden: E.J. Brill, 1960.

Foucault, Michel. Discipline and Punish: The Birth of the Prison. Trans. Alan Sheridan. New York: Viking, 1979. 
Grabar, Oleg. The Alhambra. London: Allen Lane, 1978.

Gonzales, Valérie, Beauty and Islam: Aesthetics in Islamic Art and Architecture. London: I.B. Tauris, 2001.

Larson, Jacqueline. 'What if the Object Should Shoot? - "Women of Allah" As Veiled Criticism', in: Susan Edelstein (ed.), Shirin Neshat 'Women of Allah'. 5-25. Vancouver: Artspeak Gallery, 1997.

Moore, Lindsay. 'Frayed Connections, Fraught Projections: The Troubling Work of Shirin Neshat', in: Women: A Cultural Review, vol.13, no.1, 1-17, 2002.

Naficy, Hamid,.'Parallel Worlds, Shirin Neshat's Video Works', in: Shirin Neshat, Vienna: Kunsthalle Wien and Serpentine Gallery London, 42-53, 2000.

Nasr, Sayyed Hossein Islamic Art and Spirituality. New York: State University of New York Press, 1987.

Schimmel, Annemarie. 'Calligraphy and Sufism in Ottoman Turkey', in: Raymond Lifchez (ed.), The Dervish Lodge: Architecture, Art and Sufism in Ottoman Turkey. 242-252. Berkeley: Berkeley University Press, 1992.

- Calligraphy and Islamic Culture. London: Tauris, 1990.

- Mystical Dimensions of Islam. Chapel Hill: University of North Carolina Press, 1975. Shirin Neshat. 'Women of Allah'. Susan Edelstein (ed.), Vancouver: Artspeak Gallery, 1997. Silverman, Kaja. Male Subjectivity at the Margins. New York: Routledge, 1992.

- Threshold of the Visible World. New York, London: Routledge, 1996.

Wendt, Selene. 'Beyond Orientalism', in: Shirin Neshat, Høvikodden, Norway: Heine Onstad Kunstsenter, 1999.

Yeğenoğlu, Meyda. Colonial Fantasies: Towards a Feminist Reading of Orientalism. Cambridge: Cambridge University Press, 1998.

Zabel, Igor. 'Women in Black - Islamic Fundamentalism in the Photographs of Shirin Neshat', in: Art Journal (Winter 2001), 17-25. 


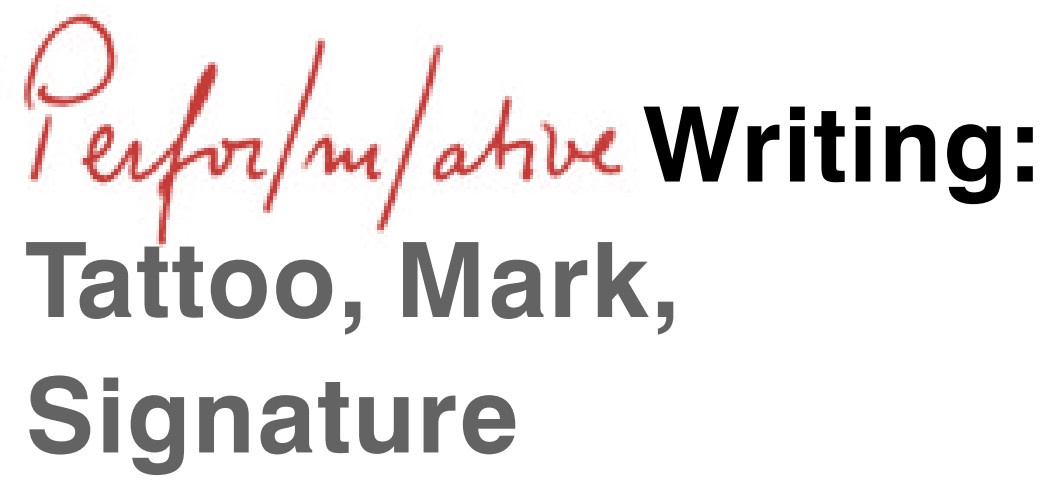

His masterpiece: an extraordinary tattoo with which he is covering the back of his wife while making love to her, from behind, having understood that such was the condition of his 'ductus'. He is seen pushing in his pin while his wife, who is lying flat on her belly, turns a suppliant and pained face towards him...

She cannot see the masterpiece she is wearing, not directly, and not without a mirror, but it subsists directly on her, at least for some time - lodged [á demeure] for a limited time, of course.

(Jacques Derrida, Monolingualism of the Other, 78)

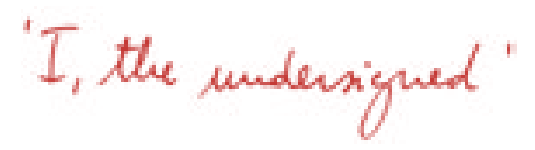

When Jacques Derrida in the early 1970s sent the written text of his lecture 'Signature Event Context' to the Association des sociétés de philosophie de langue française, he signed his text and accompanied his signature by remarking: 'That dispatch should thus have been signed. Which I do, and counterfeit, here. Where? There. J.D.' His signature is printed in facsimile in the book on the right side of this comment.
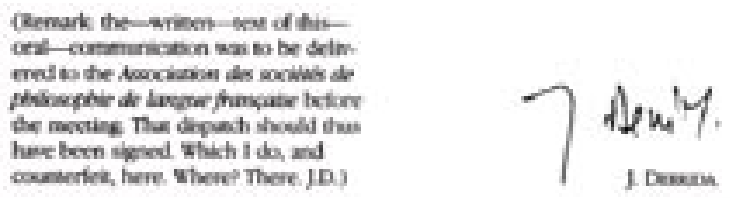
This enactment of the signature as copy gets to the heart of Derrida's critique of an all too positivist view on a metaphysics of presence. Derrida resists the idea of writing as retracing the origin, that is, the presence of the sender. For Derrida, writing has, rather, to do with absence, especially the absence of the addressee, to whom the signature is directed, but also the absence of the sender, 'from the mark that he abandons, and which cuts itself off from him and continues to produce effects independently of his presence..., indeed even after his death, his absence, which moreover belongs to the structure of all writing...' $(1988,5)$.

All writing?

The implications of this statement are already challenging for the handwritten signature because it postpones the presence of its writer's authentic and un-exchangeable physical body from a historical here and now on which the logic of the signature relies so much, towards a future in which corporeality is dissolved in the trace of writing. However, if not only the moment of performing a signature is based on corporeal presence, but if also the material to be written on is a physical body, like in the signature piece in illustration 2, how can we then understand Derrida's theory of the signature without falling back upon a philosophy of immediacy?

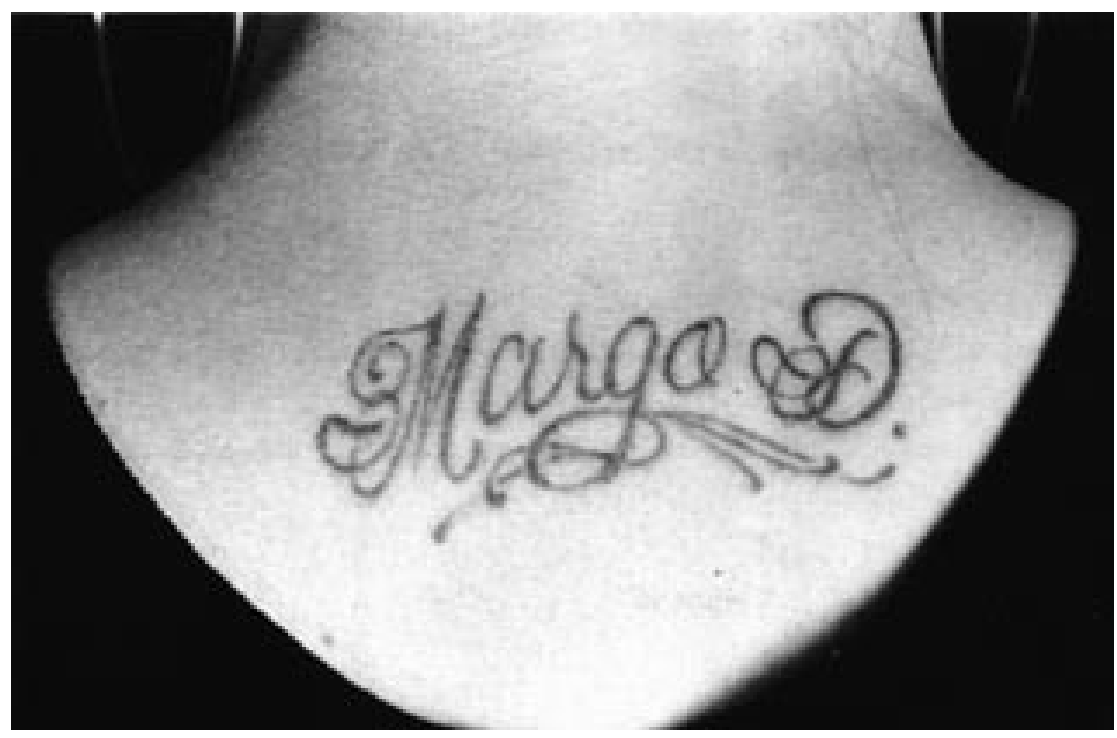

2. Margo DeMello's signature

Margo DeMello, like Derrida, is an author who signs her book; a monograph on tattooing entitled Bodies of Inscription. A Cultural History of the Modern Tattoo Community (Illustration 2). The back cover includes a photograph of the author's name, Margo D., which is said to have been tattooed by Joe Vegas onto the author's back and photographed by Vida Pavesich. The 'author's back', here, is equivalent to 
'the book's back', and yet, unlike the author's book, her body - her flesh, her skin - cannot be 'cut off from' her writing. Skin as a writing surface unfolds a semiotic process that cannot be reduced to the concept of an individual body remaining outside of the process of signification, nor can it be qualified as the material aspect of the signifier only. For the practice of tattooing, it is unavoidable that the body will intervene in the concept of writing, above all handwriting, as it seems to challenge the Derridean idea of writing as a sign that continues to produce effects independently of the author's presence, 'indeed even after his death'.

DeMello's tattooed signature piece raises yet another problem. For handwriting, and especially for the signature, ductus, pressure, and movement of the hand are culturally understood as signs reflecting individual identity. ${ }^{I}$ In the case of DeMello's signature, however, the writing on her back with its ornamental loops and curls refers to the work of a calligrapher rather than to an impulsive act of signing. It is indeed 'handmade', but executed by means of a writing machine which standardizes writing ductus and pressure into a repetitive, consistent perforation of the skin and injection of ink - a writing tool somewhere between typewriter and sewing machine. Since Kittler's Gramophone Film Typewriter, it is well known that both typewriters and sewing machines were built with great success by Remington, a company that initially specialized in weapons production. For tattooing, all three mechanisms seem vital: the violating dimension of weapons, the repetitive penetration by the needle of the sewing machine which simultaneously riddles the fabric and joins it to itself, and the mechanical act of writing performed by the typewriter. The electric tattoo machine was invented circa 1890 by Samuel F. O'Reilly to keep up with the demand of customers in his tattoo shop in Chatham Square in New York's Bowery (Govenar, 214-215). ${ }^{2}$

Moreover, the person named by the signature is not identical to the person performing the signature. Who is the 'I, the undersigned'? Who is signing whose proper name? In other words, this writing raises the question of the status of tattooer and tattooed. From its very beginning, the professional status of the Western tattooer ranges from an ordinary hairdresser or barber - when the tattooer mainly works from 'flash', pre-designed motifs displayed in catalogues or on the walls of the tattooshop - to a tattoo artist who is known for a personal style. Tattoo shops, thus seen, may resemble either a Carolingian chancellery where the scribe functions as a manus ministra, authorized to perform a pre-designed signature on behalf of the king, or a postmodern atelier where an artist creates an artwork in her unparalleled style. ${ }^{3}$ Such art tattooers are considered 'first-person' performers. The tattooed 'artwork', as an object to be written on, comes to stand in a 'thirdperson' position where she or he is named by the subject executing the tattoo. This tattooed person is not a silent object, however. She or he actively participates in the process of choosing the design to be tattooed, and influences the effects produced by the tattoo by framing it within a personal narrative which is performed whenever the tattoo is displayed: the story of why, where and when the tattoo was made, and about the pain suffered. ${ }^{4}$ As a consequence, artistic subjectivity is displayed both by the tattooed subject and by the tattooer; positions of first person and third person intermingle. 
This blurring of subjectivity challenges the foundations of the logic of the signature and of handwriting as such. A tattoo is, even more than a handwritten signature, a marking of individuality and identity. A tattoo makes the subject identifiable, incomparable and authentic. ${ }^{5}$ A tattoo is ultimately based on physical materiality, and, as such, it functions as a somatic sign referring to the irreducible presence of the tattooed body. But as a tattoo riddles the skin, it is also a 'perforation', a gap into the unitary corporeality driven by the intervention of another body with the hand of the tattooer making the inscriptions. Within this split of corporeality, not only the concept of authorship becomes problematic, but it challenges the whole idea of handwriting as a 'live' practice whereby the physical, human hand is pivotal to the production of letters. The aim of this paper is to address some key characteristics of handwriting - its emphasis on the here and now of the act of writing as well as its claim to constitute authenticity and identity - in relation to tattooing as a contemporary Western practice.
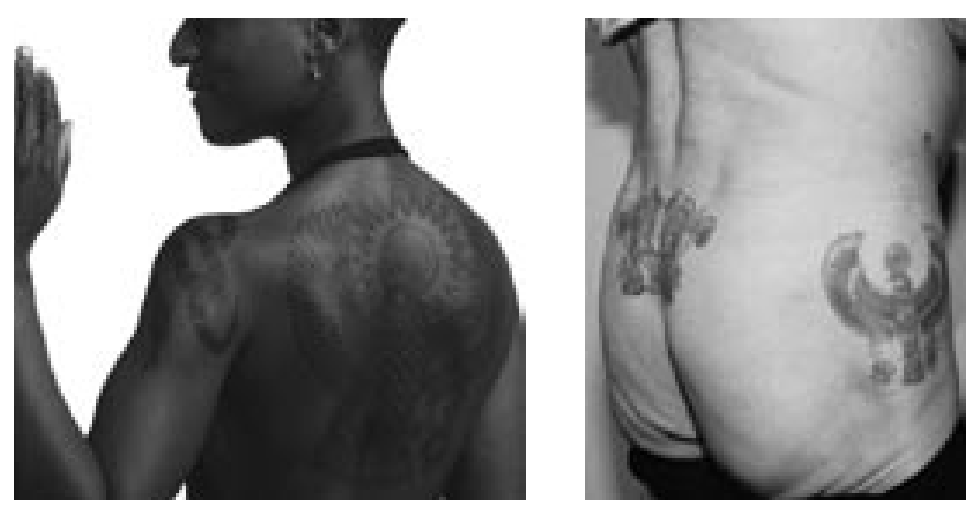

3. Patience Agbabi, photograph: Graham Brandon

4. Ian Day, photograph: Gordon Rainsford
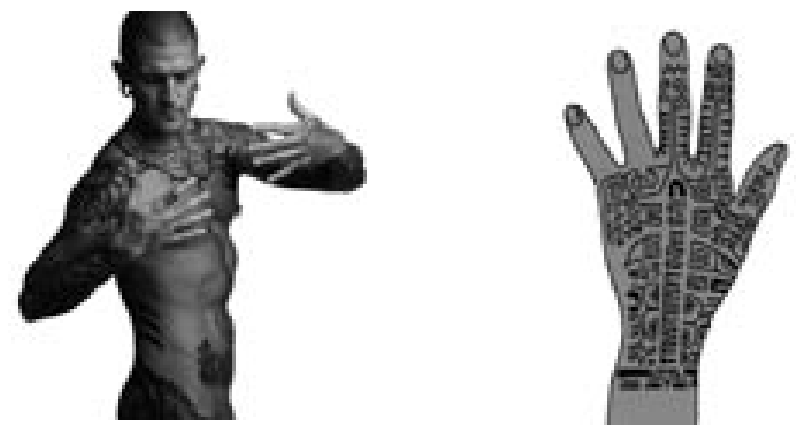

5. Tim Whitmore, photograph: Graham Brandon

6. Maori hand design 


\section{Even After Death}

The Derridean sentence 'even after his death', when adopted for the tattoo, evokes a taste of decomposition. In what follows, I will explore the corporeal dimensions of this phrase by analyzing some examples which are mostly selected from a digital online art exhibition collecting photographs of tattooed people, photographed by Graham Brandon and posted on the web by the Victoria and Albert Museum in London. Illustration 3 shows Patience Agbabi whose back is covered with a tattoo depicting the Egyptian sun god Ra, a symbol of creation and life represented as an eagle carrying the sun on his head through the sky. For this symbolic dimension, $\mathrm{Ra}$ is a favorite motif for tattoo design and is found on many bodies. When tattooed onto Ian Day's body (Illustration 4), however, this notion of creation and life gets a slightly bitter aftertaste. The condition of this tired and worn-out writing surface makes it a sign for transitoriness and death. Such dedication to death, is this not a rule inscribed in all tattoos?

Illustration 5 shows a photograph of Tim Whitmore. In the online database of the V\&A Archive, he describes the tattoo on his left hand as a version of a Maoriinspired triangular design that is part of a design from Borneo. Tim's partner wears the other half of the design and together they form one complete design. The tattoo was done by Mike from Athens as a gift to the two of them upon the anniversary of their first meeting. Thus, the tattoo on the finger, in a way, functions as a wedding ring. However, unlike a wedding ring, a tattoo cannot be removed without leaving a scar. In this context, the Derridean sentence 'even after his death' sounds like the opposite of this other phrase 'until death do us part', a dictum which is unbound from its legitimacy at the moment of death. Before death, however, this tattoo claims to establish unity - between Tim and his partner, but also between writing and the material presence of the body. Yet, and this is precisely what the Derridean critique of presence refers to, the de-'sign' on Whitmore's hand cannot be understood other than as a 'sign', as a speech act uttering 'I hereby marry', and by saying this, celebrating - or 'performing' - the marriage.

Like a wedding ring, a tattooed name is a lifetime promise and is therefore a favorite motive in tattoo art. This promise cannot be revoked, under any circumstances; it says: 'I love you forever'. In illustration 7, the list of three names 'Matthew/Adam/Lucy' is enigmatic. Does it stand for a menage a trois - be it a polygamous partnership or parents and child, though the latter would seem a bit odd because these names all have the same visual status, which would not correspond to the hierarchical relation between parents and child. Alternatively, the arrangement of these names can be read as a list of partners in order of time with two open slots at the end. When the list is full, the game is over. Whatever reading one chooses, the time vector of this tattoo is undeniable: 'I love you forever'.

This promise of 'forever' becomes even more problematic when taking into account a detail of this image that can easily be overlooked. This detail concerns less the tattoo itself than the materiality of the skin with its little spots, some of them looking like pigments, others like pustules, pimples, little bloody injuries display- 
ing the skin's vulnerability. They resemble Barthes' punctum, an element in a photograph 'which rises from the scene, shoots out of it like an arrow, and pierces me' (26). Barthes literally describes the punctum as 'this wound, this prick, this mark made by a pointed instrument... it also refers to the notion of punctuation' (26). The punctum is also a 'sting, speck, cut, little hole - and also a cast of the dice' (27). The punctum is the element which comes by surprise and which breaks with the committed, culturally trained mode of looking, which Barthes refers to as the studium. The punctum has the potential to interrupt the studium and to redirect, suddenly and unexpectedly, the process of meaning production. The tattoo's punctum concerns less the recognizable depiction, which would rather invite the interpretative examination of the studium, than the secondary markers of the almost invisible stabs, little punctures and injuries underlying the tattoo. This blessed skin finally mirrors the basic idea of what a tattoo is: a perforation of the skin, an infliction of pain, an injection of ink, a wound that heals and thus becomes writing.
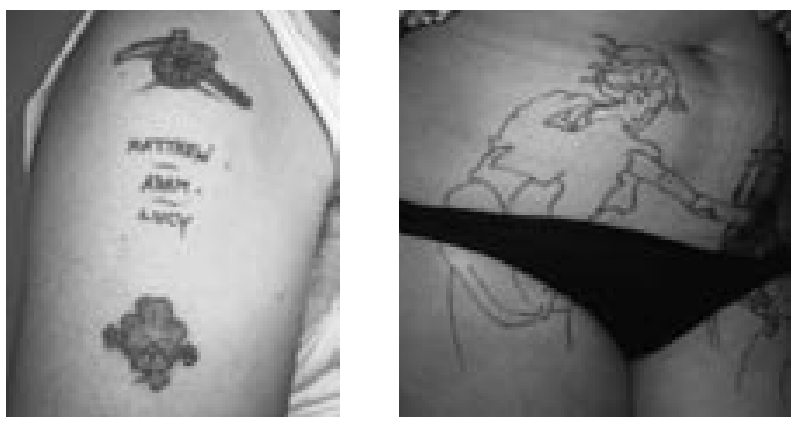

7. Richard Botheras, photograph Michaela Wyatt

8. Natalie, photograph Othello Hartley

The tattoo in illustration 8 is composed of fine black lines representing a Chinese scene. This image's punctum makes us aware of another figure that is overwritten like in a palimpsest. This secondary figure is, like the tattooed scene itself, formed by lines that do not actually belong to the tattoo and yet interfere with it. These lines are imprints from the clothes which had to be taken off to expose the tattoo to the photographer, the Internet user, you, the addressee for whom this writing continues to produce effects independent of the author's presence, 'indeed even after his death'. These imprints redirect our gaze from the tattoo, as writing, towards the skin. Likewise, the skin covered with goose bumps in illustration 9 is not just an invisible writing surface. As opposed to canvas or paper, the skin cannot deny its material mode as physical, susceptible to time, diseases, heat, and cold. A tattoo, after all, cannot be read but on a naked body. It signals that we can only feel or touch when we are naked. All these tiny little details - these pustules and pimples, these fat-folds and goose bumps - as a punctum striking us by surprise, stress our understanding of the tattooed body as a signifier by itself, emphasizing the vulnerability and nakedness of our existence. 


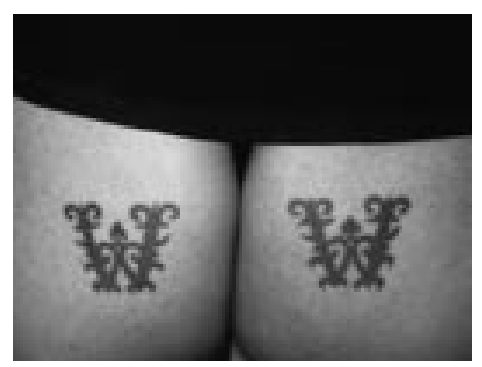

9. Beautiful strange, photograph Jan Dunning

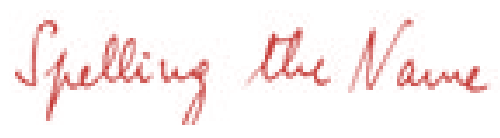

Unlike writing on paper or on a computer screen, the signifier of tattooed writing cannot be conceived as an almost invisible medium that 'immediately' links us to a signified meaning. Rather, it arrests the gaze and makes us aware that a tattoo, like ballet or theatre, takes the living body as its medium. Tattooing therefore qualifies as performance culture. 'Performance' is epistemologically studied in cultural practices such as theatres, side shows, festivities, rites, musical or dance performances - all practices that require the presence of physical bodies and thus function as singular events which, like a handwritten signature, claim to be authentic, unexchangeable and unrepeatable. The cultural role of the tattoo in Europe, from its early beginnings until the present, always resided in this display of a present body. Since the 17th century, tattooed natives from the Pacific Islands were displayed in the Western world's freak shows, dime museums and circuses. In the early 20th century, such attraction slaves were sometimes displaced by tattooed Westerners, who sold pamphlets at their performances that described them as victims of cannibal natives or other savages who had kidnapped and tattooed them.

This racist idea of tattoo as a 'naked', 'pure' and 'savage' cultural practice was soon used by Westerners to mark themselves as wild or exotic. In the 18th century, mariners and sailors tattooed themselves as an articulation of exoticism and adventure; in the 19th century, soldiers used it as an expression of courage and combativeness; and in the 20th century convicts, motorcyclists and other workingclass populations discovered it as a mode to mark themselves as marginalized outlaws. In postmodern culture the practice of tattooing still functions as an import from an exotic, wild, natural, 'original' culture. In most of these cases, the tattooed subject is inscribed into a system of classifications, into a set of norms formulated by a community marked by difference. This logic of classifying, I will argue, is not only based on a semiotics of 'skin' as a racial, sexual, and social signifier, but it is also the basic operation of the tattoo. 
Etymologically, the word 'tattoo' is derived from the Tahitian ta tau, which means 'to mark'. When Captain Cook discovered Tahiti in the 18th century while sailing for the British Navy, he described Polynesian tattooing as a sign system expressing genealogical relations (Kaeppler). Like the practice of naming in Western society, the Tahitian tattoo is a marking that comes from the outside, serving the aim of inscribing the subject into a social system. Tattooing embodies a socio-political power of locating the one marked within a context. 'I hereby baptize' is such a phrase that - as Austin argues in How to do Things With Words - by saying it, performs what it says. The name given in this rite identifies the subject. ${ }^{6}$ Naming the subject is a discursive practice that generates what it names, that does what it says. From this magic moment on, the subject is 'spelled' by the proper name - in the double sense of the word 'spelling' because identity is conjured from that very act of writing understood as treading one letter upon the other. ${ }^{7}$

Like baptizing and marrying - Austin's favorite examples - marking, tattooing, and signing are equally based on the logic of performativity. Signing is the example Derrida uses in 'Signature Event Context' in response to Austin by arguing that performativity is not reduced to a certain mode of speech act, but that any cultural utterance is performative. The signature is performative because it is a singular act, a 'life' practice grounded in repetition, since any signature cites the system of signing it derives from. 'Performativity', says Judith Butler in Bodies that Matter, echoing Derrida's discussion of performativity, 'is thus not a "singular" act, for it is always a reiteration of a norm or set of norms, and to the extent that it acquires an act-like status in the present, it conceals or dissimulates the conventions of which it is a repetition' $(1993,12)$. Unlike Derrida, who relates the signature to the future, to future readers, as the subscriber is 'always already' deferred in the gesture of signing, Butler, in Excitable Speech, emphasizes the historical dimension of performativity by arguing that a performative iterates (or 'cites') cultural norms and conventions of the past. When framed within Butler's theory, tattooing can be considered such a repetition of norms - social and racial norms marking familiarity and difference between self and other. It thus functions as a performative. The practice of tattooing can be regarded as a singular event that is grounded in the repetition of cultural norms. If the tattooed body is a marked body, it is not only marked by the difference between vulnerability and healing, between life and death, presence and absence, but it is also marked in the etymological sense of the word ta tau.

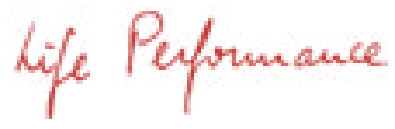

One of the greatest modern tattoo spectacles in the Western world in the 1930s was the 'Great Omi' (Illustration 10). The Great Omi, also called 'the Zebra Man' (his real name was Horace Ridler), was tattooed with bold black zebra-like stripes covering much of his body, including head and face. As the years passed, the Great Omi took to wearing lipstick and nail polish to arouse the curiosity of paying crowds. He had his earlobes stretched and pierced with ivory spikes; he had his teeth filed to sharp points, and wore an ivory tusk in his nose. He signed his pitch 
cards 'Barbaric Beauty'. (Cohen, 29; 52). In his correspondence with his tattooer George Burchett, the Great Omi complained that the more outrageous his appearance became, the less respectful he was treated by his employers. He described his most insulting experience in a small circus in France as follows: 'When I was too ill to work they painted up a nigger with white paint and put him in my place.' For the sideshow, a white man exposing black features appears to be replaceable by a black man exposing white features. Black on white, or white on black, what is the difference? ${ }^{8}$ For a conceptualization of the tattoo as writing or signature, it makes a difference, or more precisely, a 'differant' in the Derridean sense of the word. Black ink on white paper, after all, cannot be understood but as the signifier par excellence of white, literate, male-dominated civilization, which is constituted in opposition to 'the other', the illiterate, primitive, barbarian savage. 9

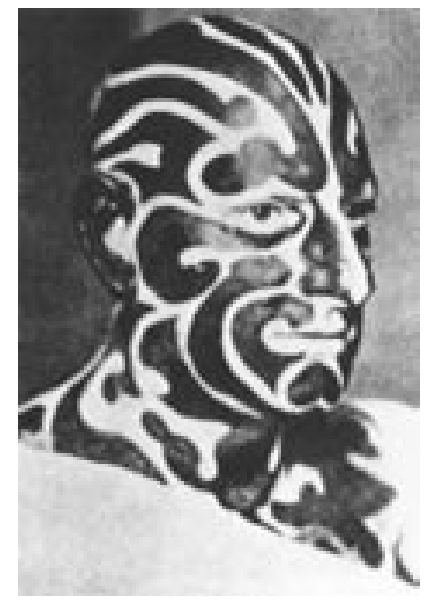

10. The Great Omi

Tim Whitmore describes his tattoos in the online database of the V\&A Archive as a mixture of Japanese, Maori, Celtic and Thai-inspired designs. The intertextual pre-texts quoted here are ornaments and symbols recalling far-away or long-ago exotic cultures. The Maori-inspired design on Whitmore's left hand, for example, refers to the New Zealand tradition to mark the Moko Master with a chiseled design used for hand and face tattooing. Although a tattoo takes the living body for its medium, these historical tattoos can still be seen, not only in drawings or photographs, but actually in the skin, not only as a performative citation in the shape of a quote on Tim Whitmore's body, but as an authentic and original performance. The reason for this presence of tattooed bodies - even after their death - resides not only in the Maori's custom of tattooing their chiefs (marking them as such) and removing and preserving their heads after death. It is also based on a macabre chapter in Western history; when Europeans began to colonize New Zealand, the dried heads of the native Maori became part of a commercial enterprise, stocking the museums of Europe and the United States with specimens of 'barbaric face-culture' (Illustration 11). ${ }^{\text {IO }}$ 


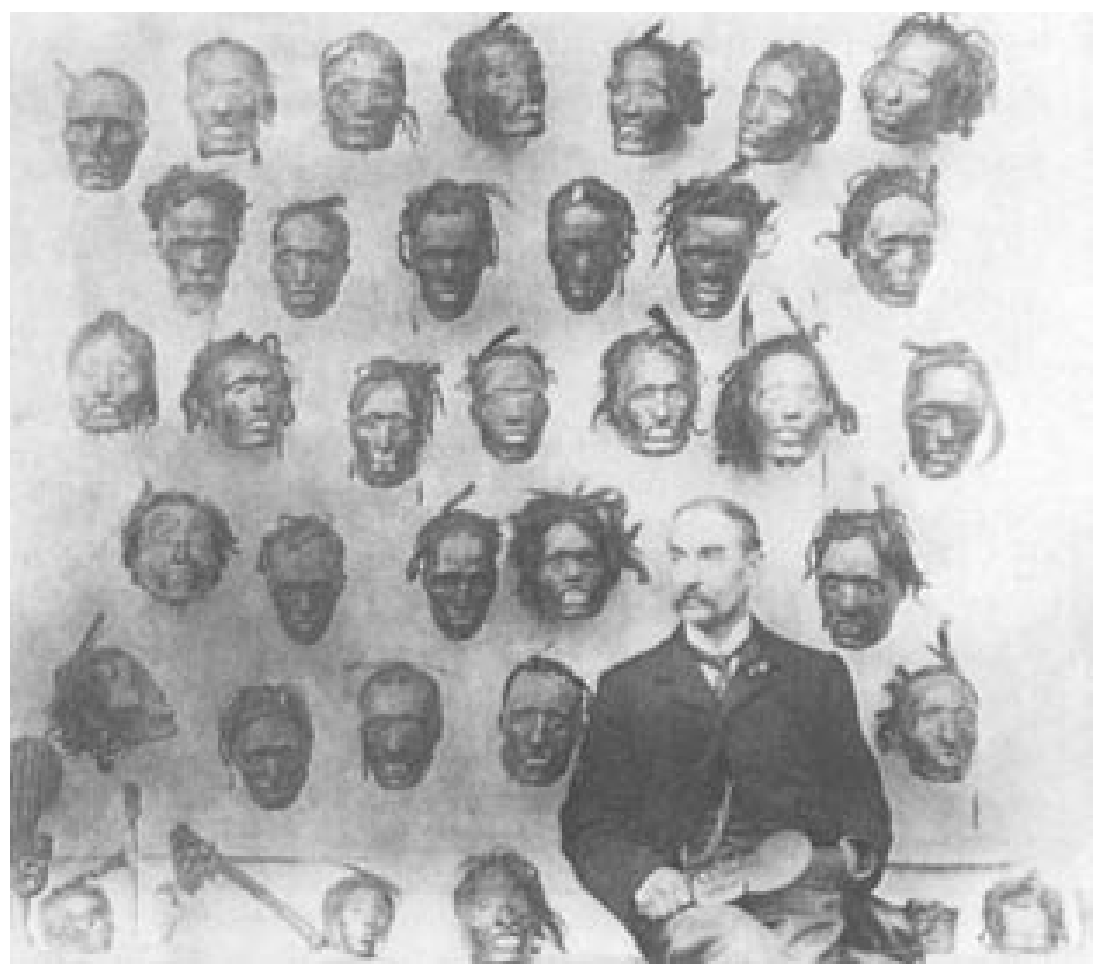

11. Maori heads

The Maori designs tattooed onto Whitmore's body are equally transferred from the South Seas to Europe, this time not in their physical originality, but as a quotation. Yet on Whitmore's white skin, these tattoos no longer qualify as fixed writing, as conventional signs functioning as coded and legible markers of political hierarchy - as they did in Moko society. As a quotation, they indeed repeat their context, but they also have the potential to break with that context. According to Derrida, this potential to break with a context is constitutive for any semiotic process: 'Every sign... can be cited, put between quotation marks; in so doing it can break with every given context, engendering an infinity of new contexts in a manner which is absolutely illimitable.' (Derrida 1988, 12; emphasis in the original). Just like a signature, the symbols on Whitmore's skin affirm their pre-text, but not without difference. Whereas in Moko society, these symbolic stripes indicate a violating act of naming and thus of labeling or fixing a person within a pre-defined political hierarchy, Whitmore's tattooed body challenges rather then affirms such social hierarchical structures. These tattoos claim to provoke a break, both with the culture of origin and with the culture of destination. In doing so, they establish a new - or, as Michail Bakhtin would say, a 'third' - space in between these cultures. 
Even though Whitmore's tattoos undeniably serve to mark the self as other, they do not necessarily qualify as a racist fixation of the other as for example the Great Omi's tattoos do. ${ }^{I I}$ Shortly before his death in 1969, the Great Omi confessed that 'underneath it all, I'm just an ordinary man'. ${ }^{22}$ Ordinary, here, means white and civilized, and civilization is what is underneath the skin. Skin, thus defined, becomes an opposite of 'mind' or 'soul', which is invisible since hidden under the surface. Unlike the Great Omi, Tim Whitmore emphasizes that his tattoos bring his deepest held beliefs - his religion, his philosophy, his being married - onto the surface and thus performs those for the other.

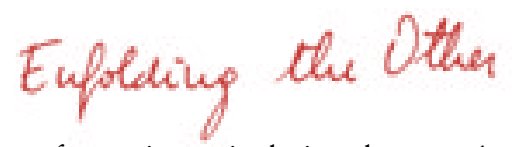

This idea of skin as a performative articulation that - as Austin would say - 'does' the self, comes close to Didier Anzieu's psychoanalytical concept of the 'skin ego'. The ego, for Anzieu, is not only located in the mind. Rather, it is an effect of cutaneous experiences and fantasies. Anzieu introduces his theory with the observation that in embryonic development, the cortex of the brain (the place where human consciousness is located) grows out of the surface of the early fetus from where it re-enters the inside of the body. This paradoxical insight that 'the centre is situated at the periphery' (9) makes him conceive of the ego as being ectoderm, leading him to call it a 'skin ego'. Hence, for Anzieu, ego and skin are not opposites - as they were for the Great Omi. On the contrary, the skin can be seen as a vessel or an envelope that contains the ego, a process defined by Anzieu as 'invagination'. He uses the metaphor of the vagina to explain the concept of 'skin ego' because the vagina is a fold in the skin that is at once on the outside and on the inside, thus externalizing the inside and internalizing the outside.

Because the skin forms the border between the inside and the world outside it has for Anzieu the potential to function as interface. The skin both protects the ego against intruders, against aggressive penetration by the other, and it is at once the (erogenous) zone where contact between self and other takes place. Skin, thus considered, can no longer be regarded as an essentialist somatic sign establishing racial, sexual, or social difference. On the contrary, it becomes a permeable velum which can be transgressed both from the inside and from the outside, thus allowing for new, hybrid identities.

The concept of 'invagination' in the sense of Anzieu allows for a more detailed analysis of tattooing and of handwriting on the whole. Like handwriting, tattoos - as the example of Whitmore's tattooed body shows - for their bearers serve as marks of identification; they claim to bring an authentic self hidden in the depths of the inside to the surface. Identity is here construed by tattooed marks that qualify the subject as individual and unique, since different from its community. In the case of Whitmore, however much these tattoos claim to be definite markers of diversity, they remain a repetition or a citation of a pre-text, however distant in time or space it may be. Whitmore may emphatically mark himself as 'non-Western', but in doing so he cannot avoid inscribing himself into another social context, 
which he quotes via the design of his tattoos. The Western self appears to be 'othered', that means 'invaginated' or penetrated by an exotic other.

This intervention is indeed productive for constituting cultural identity. On the other hand, though, it is an effect of a painful disturbance of the ego's borderline, a traumatization, or perforation, of the self by the other. For the act of tattooing, this violating dimension is physically true, as tattooing indeed involves an injury of the skin, an infliction of pain, and a flow of blood. And yet, for Whitmore, this penetration by a Moko design does not function as a violating act of naming in the sense that it fixes and labels a person in its essence. Rather, being a quote of exotic symbols on white skin, it breaks with its original cultural context by carrying it from the South Seas to Western culture, where the tattooed body challenges rather then affirms social hierarchical structures. The corporeal metaphor of the vagina reveals precisely the dialectics of tattooing as both wounding and healing where the self is simultaneously identified and challenged by the other. Both a tattoo and the vagina are at once wounded and healed like a scar. To echo Luce Irigaray, the vagina is both separated and one, both asunder and in unity, as folded genitals touching itself, 'always simultaneously the one and the other' (224). ${ }^{\mathrm{I3}}$

This double logic of being at once 'the one and the other' is also the reason why I consider tattooing both a 'perforative' and a 'performative' practice. It is 'perforative' because it physically riddles or perforates the skin. As a 'perforative' practice, the tattooed body is a singular event. Its emphasis is on the here and now, and for that reason it claims to be authentic and original. A tattoo can only be done 'live' and is, therefore, not to be repeated. And yet it is grounded in repetition, since it is a result, or a citation, of a cultural other that is entered into the self. Tattooing, marking, naming, or signing must have a repeatable, iterable form in order to be readable; to quote Derrida, 'it must be able to be detached from the present and singular intention of its production'. This citationality qualifies tattooing as a performative practice.

However much a tattoo's perforative, live dimension qualifies it as authentic, a tattoo must fail in its attempts of fixing a self as unitary, and finally identical, with itself. This is because this desired unity will always be riven by difference; it will have the shape of a scar, of which the unity is asunder, an effect of healing a wound caused by a violating act of penetrating the needle and injecting the ink as the writing fluid of the symbolic other. This scar, then, becomes a signifier for the rupture between life and death, absence and presence, inside and outside, self and other, as indicated by the somatic concept of invagination.

This idea of being at once 'the one and the other' does not only apply to the practice of tattooing, but it also holds for handwriting. Similar to the cultural role of tattooing, handwriting is often seen as a secure sign of identity. For example, the logic of the signature relies on its capacity to function as an index pinpointing at the subject of writing, and its legitimacy is based on an assumed unity between hand and writing. However, one must admit that this unity is not purely self-reflexive but is split by the (traumatizing) intervention of the other who has not only predesigned the letters to sign with, but who has also established the system of sign- 
ing. Even the absolutely singular signature is valid only as a repetition of the ritual of signing. And even though it is an absolutely un-exchangeable autographical drawing of that which claims to be the strongest bulwark of identity, the proper name, it is still split by the gap between the one naming and the one named. This other comes from the outside and yet is enfolded inside, it is at the same time the one who provides the letters to 'spell' the name, and the one to whom any handwriter directs her writing, 'indeed even after death'.

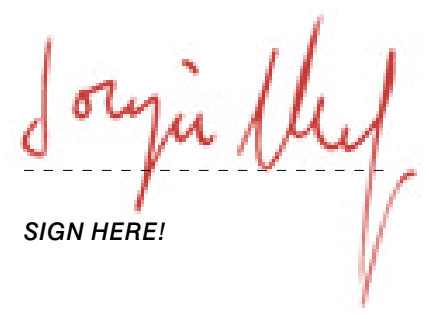


Notes

1. The assumption that handwriting can trace the writer's character is as old as the practice of handwriting itself. Prominent representatives of graphology are Camillo Baldo 1622; Cesare Lombroso 1893; and Ludwig Klages 1905-1927; see also the 'Introduction' in this volume.

2. Govenar (215) describes this tool as follows: 'The electric tattoo machine (patterned after the rotary mechanism of a sewing machine) not only quickened the process and decreased the pain involved, but facilitated greater detail and subtlety in colouration and shading.'

3. For a wonderful rich historical approach to the signature see Fraenkel's La signature. The practice of performing the royal signature is described on pages 29-30.

4. See, for example, DeMello's monograph Bodies of Inscription.

5. The problem of identifying a subject became a new issue in the 19th century, when biometrical data such as fingerprints, characteristics of the face, the walk and other body movements entered into modern criminology. See also FechnerSmarsly's article on 'Fingerprint Files' in this book. Cesare Lombroso (L'uomo delinquente. Turin, 1897) and A. Lacassagne (Les tatouages: étude anthropologique et medico-légale. Paris, 1881) also included the tattoo in their anthropological classifications of individuals as a secure signifier for a criminal subject. See Caplan, 'National Tattooing', 2000, 156-173 as well as Oettermann 1994, 58-74.

6 . This idea of a spelling force of the proper name comes close to Derrida's theorization in his chapter 'The Battle of Proper Names' in: Of Grammatology. There he argues against Lévi-Strauss's classification of the Nambikwara Native Americans as 'wild', by stating that a society that forbids the use of the proper name cannot be qualified as illiterate because they obviously know about the 'spelling' force of the name. See also Derrida's Otobiographies (1984), where he discusses the use of Nietzsche's proper name as a use 'on credit', an appraisal which also emphasizes the power structure of the proper name. 7. Juliet Fleming in her brilliant article on 'The Renaissance Tattoo' discusses the act of naming through a tattoo as highly dependent on the cultural norms that frame it. Depending on cultural norms and on naming practices, a tattoo may either appear as 'a scandalously prosthetic act of naming - one that labels, rather than divines, the essence of a person or thing', or, within another cultural order, it may 'cause identity retroactively' (82). The latter position comes close to my own analysis of tattooing as a performative act.

8. This enactment of racial difference is the central subject of an installation by Glen Lygon consisting of a diptych of two identical full-body photographs, one entitled Self-portrait exaggerating my black features, the other one Self-portrait exaggerating my white features. Photographed in high-contrast black-and-white, the miseen-scène of whiteness and blackness interrogates, as Cylena Simons puts it 'the self as a racialised subject' (141). The rhetorical power of such undecidability has been demonstrated masterfully by Paul de Man in his Allegories of Reading, where he installs his concept of undecidability by making Archie Bunker ask this famous and dangerous question: 'What's the difference?'

9. The tattoos noted by Captain Cook's crew included lines, stars, and other geometric designs, as well as figures of animals and humans. According to Lévi Strauss's classification of writing, these tattooed marks could be considered less 'real writing' than a writing of 'savage' or at best 'barbarian' people: 'The depicting of objects is appropriate to a savage people, signs of words and of propositions to a barbaric people, and the alphabet to civilized peoples [peoples polices]' (quoted in Derrida 1997, 337, footnote 9). The letters of the Roman alphabet, thus considered, generate a writing which can hardly be understood other than as a tool used to establish white dominance by means of 'spelling' white identity.

10. It appears to be a favorite practice of 19th-century museums, including the famous Natural History Museum in New York - as Donna Haraway points out - to use exhibits of foreign cultures as the 'justification for colonial domination and for maintaining the so-called natural hierarchies of race, class, and gender' (quoted in DeMello, 54).

11. Susan Benson even goes so far to ascribe authenticity to such postmodern tattoo practices: 'The identification with the primitive and the exotic is... no longer abjected, but is reconfigured as identification with the authentic, the uncommodified, the pure, in opposition to the corrup- 
tions of mainstream society; or, in the case of Japanese tattoo, with the refined aesthetic of an "ancient civilization"' (242). This assessment indeed expresses what could be called the 'tattoo dream'. But I think that this simplifies rather than explains the idea of 'authentic identity', which is, as is also discussed in the first section of this book, haunted by the troubles of the impossibility of originality, uniqueness, and singularity which also qualify handwriting.
12. See <www.tattooarchive.com/history/ great_omi.htm $>$.

13. The concept of the fold is a central one in poststructuralist epistemology. I refer to Deleuze's $A$ Thousand Plateaus (with Guattari) as well as his The Fold: Leibniz and the Baroque. Derrida in his Dissemination designs a fold that is entangled in the dissemination as dissemination. All these folds, rather than coming to a halt in the face of Luce Irigaray's binary, go on into infinity.

\section{Works Cited}

Anzieu, Didier. The Skin Ego: A Psychoanalytic Approach to the Self. New Haven: Yale University Press, 1989.

Barthes, Roland. Camera Lucida: Reflections on Photography. Trans. Richard Howard. London: Fontana, 1984.

Benson, Susan. 'Inscriptions of the Self: Reflections on Tattooing and Piercing in Contemporary Euro-America', in: Jane Caplan (ed.), Written on the Body, 234-254, 2000.

Bradley, James. 'Body Commodification? Class and Tattoos in Victorian Britain', in: Jane Caplan (ed.), Written on the Body, 136-155, 2000.

Butler, Judith. Bodies that Matter. On the Discursive Matters of 'Sex'. New York and London: Routledge, 1993.

- Excitable Speech: A Politics of the Performative. New York and London: Routledge, 1997.

Caplan, Jane (ed.). “"National Tattooing”: Traditions of Tattooing in Nineteenth-century Europe' in Caplan (ed.), Written on the Body, 156-173, 2000.

- (ed.). Written on the Body. The Tattoo in European and American History. London: Reaktion Books, 2000.

Cohen, Tony. The Tattoo. [s.1.]: Outback, 2000.

DeMello, Margo. Bodies of Inscription: A Cultural History of the Modern Tattoo Community. Durham and London: Duke University Press, 2000.

Derrida, Jacques. Of Grammatology. Trans. and with an introduction by Gayatri Chakravorty Spivak. Baltimore: Johns Hopkins University Press, 1997 (1976).

- 'Signature Event Context', in: Limited Inc. Evanston: Northwestern University Press, 1988.

- Monolingualism of the Other; or, The Prosthetics of Origin. Trans. Patrick Mensah. Stanford, CA: Stanford University Press, 1998.

- Otobiographies. L'enseignement de Nietzsche et la politique du nom propre. Paris: Galilée, 1984.

Fleming, Juliet. 'The Renaissance Tattoo', in: Jane Caplan (ed.), Written on the Body, 61-82, 2000 .

Fraenkel, Béatrice. La signature. Genèse d'un signe. Paris: Gallimard, 1992.

Govenar, Alan. 'The Changing Image of Tattooing in American Culture, 1846-1966', in: Caplan (ed.), Written on the Body, 212-233, 2000.

Grönig, Karl and Ferdinand Anton. Geschmückte Haut. Munich: Frederking \& Thaler, 1997.

Haraway, Donna. 'Teddy Bear Patriarchy: Taxidermy in the Garden of Eden. New York City, 1908-1936', in: Social Text no. II: 20-64, 1984. 
Irigaray, Luce. Ce sexe qui n'en est pas un. Paris: Minuit, 1977.

Kaeppler, Adrienne. 'Hawaiian Tattoo: A Conjunction of Genealogy and Aesthetics' in Arnold Rubin (ed.), Marks of Civilisation, 157-170. Los Angeles: Museum of Cultural History, University of California, 1988.

Klages, Ludwig. Graphologie I. Frauchiger et al. (eds.), Bonn: Bouvier, 1968.

Oetterman, Stephan. Zeichen auf der Haut. Die Geschichte der Tätowierung in Europa. Europäische Verlagsanstalt, 1994.

- 'On Display: Tattooed Entertainers in America and Germany', in: Jane Caplan (ed.), Written on the Body, 193-211, 2000.

Simons, Cylena. 'Re-presenting Representing Subjectivity: An Investigation into the Operations of Racial Difference', in: Joyce Goggin and Sonja Neef (eds.), Text, Subjectivity, Hybridity. Amsterdam: ASCA, 2000.

<www.tattooarchive.com/history/great_omi.htm>, February 15, 2003.

$<$ www.vam.ac.uk>, March 2006 
Mieke Bal is Academy Professor at the Royal Netherlands Academy of Arts and Sciences, Professor of the Theory of Literature and a Founding Director of the Amsterdam School for Cultural Analysis, Theory and Interpretation (ASCA) at the University of Amsterdam, as well as A.D. White Professor-at-Large at Cornell University. Among her many publications are: Louise Bourgeois' Spider: The Architecture of Artwriting (Chicago: University of Chicago Press, 2001); Looking In: The Art of Viewing ([s.l.]: G\& B Arts International, 2001); Quoting Caravaggio: Contemporary Art, Preposterous History (Chicago: University of Chicago Press, 1999 [2001]); Narratology: An Introduction to the Theory of Narrative (Toronto: University of Toronto Press, 1997); The Mottled Screen: Reading Proust Visually (Stanford: Stanford University Press, 1997); Double Exposures: The Subject of Cultural Analysis (New York: Routledge, 1996); and Reading 'Rembrandt': Beyond the Word-Image Opposition (Cambridge: Cambridge University Press, 1991 [1994]). She has also edited a programmatic volume The Practice of Cultural Analysis: Exposing Interdisciplinary Interpretation (Stanford: Stanford University Press, 1999). Her newest publications are Travelling Concepts in the Humanities: A Rough Guide (Toronto: University of Toronto Press, 2002) and Kulturanalyse (edited and with an Afterword by Thomas Fechner-Smarsly and Sonja Neef, trans. Joachim Schulte, Frankfurt am Main: Suhrkamp 2002). Her areas of interest include literary theory, semiotics, visual art, cultural analysis, postcolonial theory, the seventeenth century and contemporary culture.

M.G.Bal@uva.nl

Hannelore Dekeyser works at the Interdisciplinary Centre for Law and ICT (ICRI) in Leuven, Belgium. She obtained her law degree at the Katholieke Universiteit Leuven in 2001. She studied at the Westfälische Wilhelms-Universität Münster in Germany as an exchange student (2000-2001). In 2002, she obtained a DEs degree in ICT law and administration from the Facultés Universitaires Notre Dame de la Paix Namur, Belgium. At the ICRI, her main focus is the project DAVID (Digital Archiving in Flemish Institutions and Administrations). This is a project executed in association with the Antwerp City Archives and financed by the Fund for Scientific Research, Flanders (Belgium). Dekeyser researches the legal aspects of digital archiving and has a special interest in the role of digital signatures for archival purposes. Her publications include Archiveren van e-mail (Filip Boudrez, Sofie van den Eynde and Dekeyser Hannelore, 2nd ed., DAvid, Antwerp-Leuven, 2002), to be published on the DAvid website, English translation to follow.

hannelore.dekeyser@law.kuleuven.ac.be

José van Dijck is Professor of Media and Culture at the University of Amsterdam and chair of the Media Studies department. She is the author of Manufacturing Babies and Public Consent: Debating the New Reproductive Technologies (New 
York: New York University Press, 1995) and ImagEnation: Popular Images of Genetics (New York: New York University Press, 1998). Her latest book is titled The Transparent Body: A Cultural Analysis of Medical Imaging (Seattle: University of Washington Press, 2004). Her research areas include media and science, (digital) media technologies, and television and culture.

J.F.T.M.vanDijck@uva.nl

Arnold Dreyblatt is an American artist and composer who has been based in Berlin since 1983. He was recently Guest Professor in Intermedia at the Hochschule für bildende Künste in Saarbrücken and has exhibited widely, including at the Hamburger Bahnhof and at the Anselm Dreher Gallery in Berlin. Dreyblatt's artistic projects link the use of electronic media and sculptural space in posing questions on the storage of collective and individual memory.

arnold@dreyblatt.net

Thomas Fechner-Smarsly is Professor of Scandinavian Studies at the Uniwersytet Jagiellonski in Krakow, Poland. He is also active as a literary and art critic for newspapers and radio. His publications include Die Wiederkehr der Zeichen (on Knut Hamsun and psychoanalysis, Frankfurt-am-Main: Peter Lang Verlag, 1991); Krisenliteratur (on medieval Icelandic Saga-Literature as a crisis-phenomenon, Frankfurt-am-Main: Peter Lang Verlag, 1996); and, as an editor: August Strindberg: Verwirrte Sinneseindrücke: Schriften zu Malerei, Literatur und Kunst (Neuware: FINK (WILHELM), 1998) and Mieke Bal: Kulturanalyse (Frankfurt-am-Main: Suhrkamp Verlag, 2002). He has also published articles and essays on literature and media (photography, projection, microscopy), Robert Smithson, Friedrich Kittler and Vilem Flusser, the 'Wunderkammer,' art and memory, among others.

t.fechner.smarsly@uni-bonn.de

Begüm Özden Firat is a Ph.D. candidate and a guest researcher at the Amsterdam School for Cultural Analysis (ASCA) and is working on her dissertation entitled Visuality of the 'Other': Reading the Ottoman Miniature Paintings of the 17th and 18 th centuries.

B.O.Firat@uva.nl

Richard Grusin is Professor and Chair in the Department of English at Wayne State University. He received his Ph.D. from the University of California at Berkeley in 1983. He is the author of Transcendentalist Hermeneutics: Institutional Authority 
and the Higher Criticism of the Bible (Durham: Duke University Press, 1991); with Jay Bolter, he published Remediation: Understanding New Media (Cambridge: MIT, 1999). Grusin's latest book, Culture, Technology, and the Creation of America's National Parks (Cambridge: Cambridge University Press, 2004), focuses on the problematics of visual representation involved in the founding of America's national parks. He is currently working on the social, political, and aesthetic relationships among film and new digital media.

r.grusin@wayne.edu

Rembert Hüser is Assistant Professor of German at the University of Minnesota. From 1999-2001 he worked as a research fellow at the Center for the Research of Media and Cultural Communication in Cologne (project: The Hollywood Credit Sequence). From 2001-2002 he was a Visiting Professor of Film Studies at the University of the Visual Arts (Braunschweig). His fields of interests are film studies, cultural studies, and literary criticism. His forthcoming publication is entitled: Hitchcock - The First Three Minutes ([s.l.]: Legenda, Studies in Film 2, European Humanities Research Centre 2006).

huese002@umn.edu

Eric Ketelaar is Professor of Archivistics (Archival Science) in the Department of Mediastudies of the University of Amsterdam, and Honorary Professor in the School of Information Management and Systems at Monash University, Melbourne. His main research interests are the social and cultural contexts of archiving.

F.C.J.Ketelaar@uva.nl

John Mackenzie Owen is Professor of Information Science in the Department of Mediastudies of the University of Amsterdam. He previously had a career in IT research and development and in consultancy. He has been actively involved in drafting and reviewing research programs for the European Commission. His main research interest is in scholarly communication, including electronic publishing and digital libraries. He has published extensively and is editor-in-chief of the Kluwer Academic book series on knowledge management and information science.

J.S.MackenzieOwen@uva.nl

Sonja Neef is Junior Professor of European Media and Culture at Bauhaus University in Weimar, Germany. She worked as a post-doctoral fellow at the Department of Media Studies at the University of Amsterdam. Her dissertation Kalligramme: Zur 
Medialität einer Schrift. Anhand von Paul van Ostaijens 'De feesten van angst en pijn' was published in Amsterdam (ASCA, 2000). She co-edited Travelling Concepts I: Text, Subjectivity, Hybridity (Amsterdam: ASCA, 2001) and a volume of essays by Mieke Bal entitled Kulturanalyse. (Frankfurt-am-Main: Suhrkamp, 2002) as well as several articles on handwriting in the age of new media.

sonja.neef@medien.uni-weimar.de

Jeffrey Wallen is Professor of Comparative Literature at Hampshire College, and author of Closed Encounters: Literary Politics and Public Culture (Minneapolis: University of Minnesota Press, 1998). In 2004 he was a Fulbright Senior Scholar at the Freie Universität Berlin. In addition to writing about current critical debates, he has also published widely on 19th-century literature, and on autobiography and new media, and is completing a book on modes of influence in 19th-century culture.

jwallen@hampshire.edu

Michael Wetzel is Professor of Literary and Film Studies at the University of Bonn. He has studied philosophy and German literature at the Universities of Bochum and Düsseldorf (Ph.D. 1980 on 'autonomy and authenticity'). He has worked as an assistant in German literature at the University of Chambéry/France, as coordinator of the interdisciplinary research program 'Media and Literature' at the University of Kassel (1987-92), as Program Director at the Collège International de Philosophie in Paris (1992-98), as well as 'Privatdozent' at the University of Essen (habilitation 1996 on: 'The childwife as male fantasy'). His fields of interest are visual media (photography, film, video clips), gender theory, relation of text, image and voice; history of concepts of authorship and French philosophy (esp. Derrida). Book publications include Autonomie und Authentizität. Untersuchungen zur Konstitution und Konfiguration von Subjektivität (Frankfurt am Main: Bern, 1985); Die Enden des Buches und die Wiederkehr des Buches. Von den literarischen zu den technischen Medien (Weinheim: Acta Humaniora, 1991); Die Wahrheit nach der Malerei (Munich: Wilhelm Flink Verlag, 1997); Mignon: Die Kindsbtaut als Phantasma der Goethezeit (Munich: Wilhelm Flink Verlag, 1999) and Der Autor-Künstler (Frankfurt-am-Main: Suhrkamp Verlag, 2005).

ml.wetzel@gmx.de 
Page 6 The 'please-do-not-write-by-pen'-sign, photograph: Sebastian Grevsmühl

Page 11 Jefferson's writing machine, www.officemuseum.com/copy_machines.htm

Page 14 The Leonardo-Right to Left web site, http://www.mos.org/sln/Leonardo/write. html

Page 16 Screen capture from Microsoft screensaver Sports, Windows 98

Page 26 Anne Frank's handwriting, in Hardy's summary of the report of the Gerechtelijk Laboratorium in Rijswijk / Netherlands (150-151)

Page 36 Armando, Drawing, 1983; pencil on paper, $18 \times 13 \mathrm{~cm}$. in Ernst van Alphen, 1997, 142

Page 38 Anne Frank Diary, page 20, October, 1942, photograph: Allard Bovenberg, Anne Frank Huis Amsterdam

Page 41 Showcase with Anne Frank's Diary, photograph: Sonja Neef

Page 103 MPAA/RIAA Letter, http://mpaa.org/anti-piracy/press/2003/2003_02_13.pdf

Page 104 MPAA/RIAA Sample Memo, http://mpaa.org/anti-piracy/press/2003/2003_02_ 13.pdf

Page 105 Citibank Letter, http://www.citigroup.com/citizen/community/data/citizen03_ en.pdf

Page 107 CitibankPrint ads

Page 140 Arnold Dreyblatt, document \#1

Page 141 Arnold Dreyblatt, Document \#100

Page 145 Arnold Dreyblatt, T-Mail

Page 167 Saul Bass, title sequence, A Personal Journey with Martin Scorsese Through American Movies, GB 1995, dir. Martin Scorsese / Michael Henry Wilson, prod. British Film Institute / Miramax

Page 170 Duel in the Sun, USA 1946, King Vidor

Page 174 Alfred Hitchcock commemorative stamp, 32-cent, us Postal Service, Series: Legends of Hollywood, 1998

Page 175 What's on a man's mind?, artist unknown, year unknown

Page 183 Sigmund Freud, Notiz über den Wunderblock, www.loc.gov/exhibits/ freud/ freudobj.html

Page 184 Keyboardless PDA

Page 189 Pompeii fresco

Page 202 Frontpage Süddeutsche Zeitung, in Holzer, 83

Page 203 Jenny Holzer, 'Lustmord', in Holzer, 96

Page 207 Shirin Neshat, I Am Its Secret, 1993. RC print \& ink, photograph: Plauto. Copyright Shirin Neshat 1993. Courtesy Gladstone Gallery, New York

Page 208 Shirin Neshat, Rebellious Silence, 1994. B\&W RC print \& ink, photograph Cynthia Preston. Copyright Shirin Neshat, 1994. Courtesy Gladstone Gallery, New York

Page 221 Jacques Derrida, 'Signature Event Context', 21

Page 222 Margo DeMello, Bodies of inscription, back cover

Page 224 Patience Agbabi, photograph: Graham Brandon, http://www.vam.ac.uk/vastatic/ microsites/tattoo/images/fullsize/gb22.jpg

Page 224 Ian Day, photograph: Gordon Rainsford, http://www.vam.ac.uk/vastatic/ microsites/tattoo/images/fullsize/gr4-3.jpg

Page 224 Tim Whitmore, photograph: Graham Brandon, http://www.vam.ac.uk/vastatic/ microsites/tattoo/images/fullsize/gb11.jpg

Page 224 Maori hand design, in Grönig and Anton, 95

Page 226 Richard Botheras, photograph: Michaela Wyatt, http://www.vam.ac.uk/vastatic/ microsites/tattoo/images/fullsize/mw8-12.jpg

Page 226 Natalie, photograph: Othello Hartley, http://www.vam.ac.uk/vastatic/microsites/ tattoo/images/fullsize/oh2-9.jpg

Page 227 Beautiful strange, photograph: Jan Dunning, http://www.vam.ac.uk/vastatic/ microsites/tattoo/images/fullsize/jd7-15.jpg

Page 229 The Great Omi, http://www.tattooarchive.com/history_images/omi_cape_ wm.jpg

Page 230 Maori Heads, in Cohen, 56 


\section{Index of Names}

Aarseth, E.J. 56, 74,

Allison, Deborah 165, 178

Alphen, Ernst van 42, 46-48, 241

Anzieu, Didier 231, 235

Arms, W.Y 74

Assmann, Aleida 193

Assmann, Jan 190, 193

Austin, John L. 9, 47, 96, 97, 228, 231

Bachelard, Gaston 155

Bal, Mieke 15, 31-34, 46-48, 163, 197, 198, 204, 205, 237, 238, 240,

Balio, Tino 178,

Barnouw, David 45-48

Barthes, Roland 40, 216, 219, 226, 235

Bass, Elaine 165, 174, 175

Bass, Saul 164, 166, 168, 172, 177-179

Baudrillard, Jean $98,101,110,114$

Benjamin, Walter 10, 15, 18, 31, 34, 46, $47,50,54,57,59,155,190,193,195$, 198, 200, 201, 205, 216, 219

Benson, Susan 234, 235

Birkerts, S. 62, 74

Bissinger, Manfred 30, 46, 47

Blanchette, Jean-François 85,89

Bolter, Jay David $12,13,18,79,114,117$, 129, 133, 190, 193, 239

Bordwell, David 166, 168, 173, 178

Boudrez, Filip 84, 85, 89, 90, 237

Bowker, Geoffrey 191, 193

Bradley, James 235

Brouwers, Ton 46, 47

Bryson, Norman 47, 48, 197, 198, 204, 205

Butler, Judith $47,228,235$

Caplan, Jane 234-236

Cheung, Charles 131

Christie, Ian 179

Cohen, Marshall 179

Cohen, Tony 229, 235

Compagnon, Antoine 155, 163

Cullens, Chris 179

Czichos, Horst 45, 48

Damasio, Antonio 189, 194

Damisch, Hubert 154, 163

Day, R.E. 66, 74

De Niro, Robert 178

Decker, William 122, 133

Dekeyser, Hannelore 12, 89, 237

DeMello, Margo 222, 234, 235, 241

Derrida, Jacques $9,15,18,29,43,47,48$, $52-55,59,96,114,118,133,143,149$, 183-186, 188, 190-192, 194, 197, 205, $221,222,228,230,232,234,235,240$, 241
Didier, Beatrice 90, 121, 133

Didi-Huberman, Georges 56, 59, 156, 163, 197, 199, 205

Dijck, José van 14, 37, 45, 46, 48, 216, 237

Domarus, Max 25, 48

Doubrovsky, Serge 159, 163

Douglas, Kirk 164

Dreyblatt, Arnold 14, 134, 141, 142, 147, $149,183,194,238,241$

Duchamp, Marcel 12, 56, 57, 59

Duff, A.S. 73,74

Dumortier, Jos 78, 79, 89, 90

Durgnat, Raymond 172, 179

Durlacher, Jessica 33, 41, 48

Eecke, Patrick van $77-89,90$

Ernst, Wolfgang 45, 48, 187-189, 194

Eynde, Sofie van den 85, 90, 237

Fechner-Smarsly, Thomas 16, 237, 238

Fernback, Jan 132

Firat, Begum 17, 238

Fleischer, Dave 176, 179

Fleming, Juliet 234, 235

Fohrmann, Jürgen 187, 194

Foucault, Michel 45, 48, 186, 194, 213, 215, 219

Fraenkel, Béatrice 18, 235

Frank, Anne 12, 23-26, 28, 30, 32-35, 37, $38,40-42,45-49,118,124,126,131$, 132,241

Frank, Otto 24, 32, 43, 46

Franke, Werner 45, 48

Freud, Sigmund $31,46,48,118,163,168$, 169, 176-179, 183, 184, 194, 205, 241

Galton, Francis 199

Gardies, André 172, 179

Gibbons, Cedric 178

Gide, André 133

Ginzburg, Carlo 152, 158, 161, 163, 199, 205

Gobert, Didier $78,80,90$

Goldwyn, Samuel 172

Goodman, Nelson 10, 18, 28, 48

Govenar, Alan 223, 234, 235

Graham, S. 74, 75

Greenberg, Joel 179

Gross, A.G. 63, 74

Grusin, Richard 12-14, 18, 97, 114, 117, $129,133,238$

Gunning, Tom 177

Halm, J. van 73,75

Hamilton, Charles 25, 31, 48

Hamon, Philippe 163

Haraway, Donna 234, 235

Hardy, J.J. 30, 48, 241

Harmon, J.E. 74

Harnad, S. 62, 74, 75

Harper, Richard 125, 126, 133

Harrington, Ralph 177, 179 
Haskin, Pamela $168,177,179$

Hayles, Katherine N. 15, 18, 109, 115, 131

Heidegger, Martin 11, 18, 53, 59

Heidemann, Gerd 24, 30, 47

Henke, Josef 25, 31, 45, 48

Herring, Susan 125, 131-133

Higham, Charles 171, 179

Hirsch, Marianne 46, 48

Hitchcock, Alfred 166, 173, 174, 178, 179, 239, 241

Hitler, Adolf 12, 23-25, 27-30, 32, 35, 43, $45,46,48,49,131,135$

Holzer, Jenny 197, 202, 203, 205, 241

Hörisch, Jochen 11, 18

Hüser, Rembert 239

In 't Veld, N. 45

Irigaray, Luce 232, 236

Jenkinson, Hilary 45, 48

Jennings, N.R. 74, 111, 112

Kaeppler, Adrienne 228, 236

Kafka, Franz 138, 149

Kalmus, Natalie 171

Kant, Immanuel 51, 59

Katsch, Ethan 89, 90

Kawakami, Yoshiro 123, 133

Kawaura, Yasuyuki 123, 133

Kernan, Lisa 177, 179

Ketelaar, Eric 16, 43, 46, 48, 131, 187 , 188, 191, 193, 194, 239

Kirby, Lynne 177, 179

Kirschenbaum, M. 73, 75

Kittler, Friedrich A. 10, 18, 131, 133, 168, 179, 238

Kitzmann, Andreas 117, 131, 133

Klages, Ludwig 18, 234, 236

Klein, Yves 201, 205

Kling, R. 74, 75

Kubrick, Stanley 166

Kujau, Konrad 24, 25, 30, 31, 47, 49

Leibniz, Gottfried Wilhelm 56, 235

Leonardo da Vinci 18, 19

Lerner, Paul 179

Levy, David M. 74, 75, 189, 195

Levy, Emanuel 179

Libon, Olivier 85, 90

Lubitsch, Ernst 174

Lyotard, Jean-François 59, 154

Mackenzie Owen, John 12, 72, 73, 75, 193, 239

Macneil, Heather 77, 90, 193, 194, 195

Mallon, Thomas $121,132,133$

Mancini, Giulio 152

Manovich, Lev 176, 179

Martin, Henri-Jean 74

Marty, Eric 121, 132, 133, 174, 178

Marx, Karl 48, 155

Mast, Gerald 179

McGann, J. 73, 75
McGim, G. 75

Melville, Herman 138, 149

Mettner, Michael 179

Micale, Mark S. 179

Milne, Esther 125, 133

Mitchell, W.J.T. 35, 46, 48, 157

Montero, Etienne 78, 80, 83, 90

Mulvey, Laura 177, 179

Neef, Sonja 12, 16-18, 48, 73, 95, 99, 118, 131, 133, 216, 236, 237, 239, 241

Nussbaum, Emily 128, 129, 131-133

O'Reilly, Samuel F. 223

O'Toole, James M. 11

Oetterman, Stephan 236

Olson, David R. 73, 75

Ong, Walter J. $65,75,118,133$

Paech, Joachim 56, 59

Peirce, Charles Sanders 163, 197, 198, 205

Pfeffer, Fritz 33, 34

Picker, Günther 45, 49

Piggott, Michael 122, 133

Pintens, Walter 77,90

Plakins Thornton, Tamara 18,19

Plato 185

Pomian, Krysztof 24, 35, 46, 49

Popper, Karl 73, 75

Poster, Mark 190, 195

Preminger, Otto 166

Price, Billy 49

Proust, Marcel 150-153, 155-158, 161, 163,237

Quickenborne, Marc van $77-79,89,90$

Reddy, M.J. 66, 75

Reidy, M. 74

Ricoeur, Paul 188, 195

Rin Tin Tin 176

Roberts, R.J. 74, 75

Rockwell, G. 74,75

Romney, Jonathan 178, 179

Rössler, Beate 47, 49

Rousseau, Jean Jacques 159

Rowland, F. 67, 75

Rühle, Gerd 49

Rusch-Feja, D. 74, 75

Ryall, Tom 178, 179

Sarris, Andrew 166, 168, 178, 179

Schatz, Thomas 178, 179

Schwartz, Hillel 49

Scorsese, Martin 15, 164-166, 168-172, 174-179, 241

Sedgwick, Eve Kosofsky 158, 163

Selznick, David O. 171, 177, 179

Sevenant, Ann van 185, 195

Shadbolt, N.R. 74

Shields, Jonathan 164

Siemens, L. 74,75

Silva, Teresien da 24,49 
Simmon, Scott 179

Simons, Cylena 189, 234, 236

Staiger, Janet 166, 178

Star, Susan Leigh 191, 193

Starret, Bob 84, 90

Stewart, Martha 136, 137, 149

Stoler, Ann 43, 186, 195

Strachey, James 179

Stroom, Gerrold van der $38,46,48,49$

Svedjedal, J. 68,75

Talbot, W.H.F. 54

Tanja, Jaap 30, 49

Taylor, Alex 125, 126, 133, 195

Taylor, Hugh 187, 190, 193, 195

Thiel, Sh. 66, 75

Thompson, David 175, 179

Thompson, J.B. 67, 75

Thompson, Kristin 166, 178

Tono, Yoshiaki 57, 59

Trevor-Roper, Hugh 25, 45

Triesault, Ivan 164

Trilling, Lionel 51, 59

Verheyden-Jeanmart, Nicole 78, 90

Vernet, Marc 178,179

Vidor, King 172, 173, 179, 241

Viegas, Fernanda 125, 133

Wagner, T. 74, 75, 201

Wallen, Jeffrey 14, 134, 240

Weaver, W. 73, 75

Weller, A.C. 74,75

Wetzel, Michael 12, 240

Wills, David 53, 59

Wilms, Wilfried 80,90

Wilson, Michael Henry 165, 174, 178, 179, 241

Wood, Helen 46, 49

Wyler, William 166

Yakel, Elizabeth 122, 133, 187, 195

Yamashita, Kiyomi 123, 133

Zhang, Jane 131

Žižek, Slavoj 46, 49

Zwijnenberg, Robert 18,19

\section{Index of Terms and Concepts}

address $95,96,114,171$

address (verb) 9, 71, 224

aesthetic discourse 158

allography, allographic 11-13, 28, 29,

$39,63,156$

alphabet 10, 214, 215, 219, 234

anachronism 32

animation 175, 176, 178

archeion 186

archivalization 43, 188-192

archive (canonical archive) 43, 45-47, 67, $70,122,127,134,136,139,143-148$,

164, 184-187, 189, 190, 192, 199,

archive, archiving $16,43,61,69,72,87$, 183-188, 190-193, 237, 239

archivization $43,185,188,190-192$

aura, auratic $10-12,30,31,42,46,50,54$, $122,155,198,200,201,203,216,217$

authenticity, authentic, authentication 7 -

$12,15-18,23-25,28-33,35,37,40-43$,

$45,46,50-52,54,56,57,60-64,66-72$,

$76,77,80,82-84,86-88,96,97,118$,

$119,129,137,143,146-148,151,161$, $162,188,189,198,199,203,204,215$, $216,222,224,227,229,231,232,234$, 235,240

author, authorship $9,12,15,40,42,50$, $53,56,57,60-62,64-71,73,74,78-81$, $83,87,105,106,117,119,122,132$, $138,158,159,164,168-170,172,173$, $186,190,215,222,224,237$

autograph, autography $9,10,12,18,29$, $30,96,168$

ballpoint pen 12

biography $135,136,138,144,146$

blackboard 7,15, 174

blog communities 120,129

blogging 116, 120, 123-129, 131, 132

blogosphere $120,127,129,132$

blood 17, 160, 196-201, 203-205, 216, 232

body $15,17,29,31,45,55,67,71,95-97$, 101, 106-109, 114, 118, 119, 131, 160, $190,196,199-202,206,209,210,212-$ $217,219,222-232,234$

calligraphy, calligraphic, calligrapher 8 , 73, 207, 209-219, 223

certification, certifying, certified 23,25 , 28-30, 32, 43, 45, 60, 61, 69-74, 81, 89, 101

character $15,23,28,31,35,47,50,67$, $73,81,102,109,118,120,155,158$, $159,173,176,197,198,210,211,214$, 234

citationality $47,96,97,232$

collecting $31,44,46,134,188,225$ 
computer $7,12,15-17,62,63,73,83-85$, $89,97,100,109,116,119,124,131$, $140,146,190,227$

conduit model 66

consent $78,79,83,100$

copy $8,9,11,12,17,23,28,29,45,50$, 60-62, 64, 67, 69-71, 73, 78, 79, 142,

189, 190, 197, 199, 200, 203, 204, 222 copyright $61,62,68,104,106,112$

corporeal 15, 29, 42, 46, 215, 222, 225, 232

counterfeit $9,30,32,221$

cross-cultural $210,211,213,214,217$

cult $34,35,200,217$

custody 23, 45

deconstruction 53,56

detail $18,40,45,46,151,152,155-157$, $159,160,225,234$

diary, diaries, diary writing $8,12,14,23$ 26, 28-35, 37, 38, 40-43, 45-47, 49, $116-130,132,133,172,191,241$

diegesis 170

différance, différant 56,57

digital $7-9,12,13,18,29,30,60-77,80$ $89,95-103,105,106,108-113,116$, $117,119-123,125-129,131-133,136$, 144, 174-176, 185, 189, 190, 193, 225, 237-239

digital cinema 175

digital records 86,189 ,

directive $82,83,86,89,103$

disembodiment $15,16,131$

dis-figure $152,156,157,160,161$

DNA 102

Drawing $10,35,50,52,55,57,58,112$, $164,176,196,197,233$

dream content 169

ductus 221,223

electricity, electric $12,223,234$

electronic signature 76, 80-87, 89, 96, 98, 101-103

Email 13, 82, 95, 96, 99, 101, 114, 123, $124,145,191,193,237$

equivalence, functional 80

ESIGN 100, 101, 103, 114

e-signature directive $82,86,89$

European Union 76, 82, 103

Evidence 24, 28, 29, 39, 76-79, 82, 84, 87, $136,148,186,188$

exposition, exposure $14,32-34,43,76$, 126

Farsi 207, 209, 210, 212, 214

Fax 12, 114

fetish, fetishism 30-32, 35, 39, 43, 46, 179

figuration 154-157, 160, 214

film $8,12,13,15,18,30,42,57,110-112$, 118, 124, 164-166, 168-179, 201, 207, $218,219,223,239,240$ film-within-a-film 166

fingerprint 16, 81, 197-199, 201, 216, 234

flatness (flat-writing) 151-157, 160-162

focalizer $154,155,158$

fonts 65

forgery, forgeries $9,24,25,29,30,43,45$, $79,80,83,108$

gaze $40,47,55,154,206,209,215,218$, 226,227

gift $58,114,125,126,225$

graphology $18,118,234$

grapho-psychology 15

hand (left hand, right hand) 7, 8, 12, 13, $15-18,29,31,35,52-55,57,58,61,63$, $78,80,95,96,99,101,108,109,117-$ $119,123,131,138,142-148,152,164$, 169, 174-176, 185, 190, 196, 204, 215, 223-225, 229, 232, 233

Holocaust 23, 33-35, 39, 42, 46, 187, 205

Hurufism 204, 219

hybrid, hybridity $13,116,124,126,127$, 129, 130, 132, 164, 209, 214-216, 231

hypermedia, hypermediated 111

hysteria 169,177

icon 56, 57, 95, 152, 197, 198, 204

identity, identification $8,26,47,56,63-65$, $67-71,74,79,81,83,89,99,102,103$, $105-114,121,124,125,136,138,139$, $143,145,147,148,154,157,160,171$, $199,214-216,223,224,228,231-235$

image $13,29,34,35,40,44,47,53-56,97$, 99-101, 103, 106, 108-111, 113, 148, $151-157,159,160,164,171,172,175$, $177,189,197,201,206,209-212,214-$ 218, 225

image-text (image-writing) 13, 151

immediacy $14,55,66,98,125,185,190$, 222

impression 50, 178, 183, 184, 186, 188, 192, 196, 197, 204, 209

imprint, empreinte $16,55,148,170,197$, 201

index $39,52,57,96,151,152,196-198$, 201, 204, 215, 232

information chain 61

infra-mediality 56,57

inframince 56

ink $17,117,137,160,197-201,203,206$, $210,215,216,223,226,229,232$

integrity $78,80,82-87$

interface 114, 231

Interlink Electronics $101,102,114$

Internet $7,62,63,65,69,74,81,99,100$, $103,116,117,119,122-125,127-129$, $138,146,226$

invagination 231, 232

Islam, Islamic 207, 209-214, 218-220 
iteration, iterable, iterability $8-10,28,29$, $32,41,44,50,52,54,55,96,97,106$, 190,323

keyboard $10,12,13,17,62-64,119$

last will $76-78$

legal value $82,87,89$

legibility $63,153,155$

letter $8,34,35,37,40,65,73,95-99,104$, $105,119,124,154,169,191,192,214$, 215,228

line $7,12,29,33,52,54,69,79,154,172$, $196,197,214$

LiveSignatures 99, 101

logo 176, 177

manuscript $28,29,34,38,61,118,193$, 210,211

materiality $9-11,13,14,28,31,42,57,95$, $96,117-121,127,129,131,210,211$, $213,215,216,224,225$

memory $12,23,32,35,42,43,53,57,64$, $110-112,117-119,121,126,127,129-$ $131,134,152,165,184,185,188,189$, $192,211,214$

micro-characteristics 26,28

microfiche 12

microfilm 136

migration $15,84,85,136,138,145$

musealization 43

museum 24, 25, 32-35, 37, 39-43, 45, 46, 118

Muslim 209-215, 217-219

mystic writing-pad, Wunderblock 118 , 174, 183-185,

network presence 69,71

nostalgia 170

notary 78,80

online newspapers 66

Optical Character Reading (OCR) 8

original, originality $8-12,17,23,28-32$, $38,42,43,45,50,52,54-58,60,61,64$, $67,69-71,73,74,78-81,83-88,96,97$, $117,118,129,136,138,139,142-144$, $147,148,151,189,190,193,199$, $201,204,216,227,229,230,232,234$ painting $10,29,52,55,150,152,154$, $156,157,176,197,211$

paper $57,65,76,78-80,84,86,100,102$, 116-121, 123, 125-129, 131, 132, 136, $142,147,148,150,151,155,171,177$, 183-186, 189, 190, 192, 193, 196, 201, 203, 224, 226, 227, 229

paratext 168,169

parchment 186, 190, 196

PC (Tablet PC) 8, 13, 185, 190, 192

PDA 185, 190, 192

PDF file 104, 105 pen $7,11,12,30,65,116,118,127,150$ $153,164,185,196,198,202,203,215$, 216

pencil $12,37,185,196,197$

pentagraph 11

perception $53,54,68,153,157,158,184$, 189

performance $25,28,42,97,125,144,146$, $201,211,215,227,229$

performativity $47,57,228$

performativity, performative $9,42,43,55$, $142,228,229,231,232,234$

photocopy 136

photography, photographic $8,12,17,42$, $46,54,55,59,105,153,157,160,161$, $199,207,210-212,215-217$

physiognomy 173

pictura 173

points of identification 26

possessory credit 173

premediation, premediate, premeditated 96-101, 108, 110-114

presence $9,10,11,15-18,29,31,34,35$, $41,42,51,53,54,58,63,69,71,78$, $79,83,95,101,123,124,137,138$, $143,146,152,157,174,196-198,201$, $216,222-229,232$

preservation $29,85-87,129,147$

primal scene $169,170,177$

print $9,29,38,42,46,61,62,67,69,84$, $96,105,107,108,117-119,122,125$, 129, 196, 197, 201, 202, 216

privacy $37,119,121-123,126-130,200$ projection $7,40,57,154,155,169,171$, 172

proper name $39,223,228,233,234$

public key $81,82,101$

publishers $62,69,71,74$

punctum 40, 226

quill 12

railway spine 177

readability $15,35,39,150,151,156$

reading $35,53,62,65,68,69,71,72,121$, $124,140,144,145,147,151-153,165$, $169,190,197,198,204,209,210-212$, 214,225

reading as a collective experience 60,67 , 68

rebus 169

remediation $9,13,62,96-99,102,103$, $105,106,108-110,117,131,138,139$, $142,146,193,212$

reMediation 13,14

reproduction, replicable $7-10,12,46,50$, $52,54,57,60,64,138,139,142,148$, $185,199,200,202,203,211,216$, scanner 8,12 
school $28,37,174$

scientific communication $60,63,67,72$, 73

screen $16,17,57,65,84,106,111,116$, $119,126,164,165,168-171,174,185$, $189,190,209,218,227$

screensaver $15,16,18,19,241$

seal 77,81

self publishing $62,69,70$

self-reflexivity 156

semiophores 24, 32, 35, 46

SIG file 95-97, 99, 114

sign $7-9,12,15,17,24,34,53,55,78$, $100,101,111,118,120,145-147,151-$ $154,157,161,173,174,197,198,204$, $212,213,215,223-225,228,230-232$

signature $9,11,14,15,18,29,47,53-55$, 57, 63-65, 69, 72, 76-87, 89, 95-103, $105,106,108-114,120,121,145,150-$ $158,160,161,164-166,168,169,172$, $173,176,196-199,215,216,221-224$, 227-230, 232-234

signature, digital or electronic $76,80-87$, $89,96,98,101-103$

signet 77

simulacrum $50,98,190$

singular, singularity $9,10,17,28-32,47$, $55,69,186,190,199,201,227,228$, 232, 233, 235

SMS 124, 126, 191-193

software $74,84,85,97,99,100,101,111$, $120,121,125-128,131,185,189$

speech act $33,40,42,142,158,225,228$

standardization, standardized $7,8,10,13$, $63,64,66,71-73,119$

studium 226

stylus $12,13,184,185,190$

subjectivity $11,46,53,63,72,161,223$, 224

subscriptio 173

surplus matter 159

tablet PC $8,13,185,190,192$

tattoo $17,108,202,221-229,232,234$,

235

testament $76-78$

trace $11,13,15,17,29,31,35,40,52,54$, $55,57,119,122,135,136,138,151$, $157,159,160,174,188,192,196-199$, $204,215,222,234$

translation $43,57,84,116,193,210,212$, 213

trauma 33,177

typeface $8,168,172$

typewriter $7,8,10,18,29,62-65,71,73$, $118-120,131,223$ unique, uniqueness $7-11,15,16,28,29$, $46,50,54,63,69,72,78,80,81,96$, $97,102,106,108,123,146,148,155$, $161,200,215-217,231,235$

unreadability 210, 212

veil, veiling $55,206,209,210,212-214$, 216, 217, 219

voice $13,18,33,35,40,101,106-108$, $122,214,217,240$

vorhandenheit 53,54 weblogs $14,46,123,125-129,131,132$ will $76,78,79,89$

witness testimony 77

word-processing 119, 138

World Wide Web 123, 127, 132

writer $9,11,16,29,31,37,47,63,65,66$, $71-73,96,119,126,158,190,216,233$ writing pad $118,174,184,185$

Xerox 8, 11, 12, 28, 29 
